\title{
Summaries of FY 1995 Research in the Chemical Sciences
}

\author{
U.S. Department of Energy \\ Office of Energy Research \\ Division of Chemical Sciences
}

This report was compiled for the Office of Energy Research from project summaries contained in the Research-In-Progress (RIP) database of the Office of Scientific and Technical Information, Oak Ridge, Tennessee. The RIP database describes new and ongoing energy and energy-related research projects carried out or sponsored by the Department of Energy. 
Available to DOE and DOE contractors from the Office of Scientific and Technical Information, P.O. Box 62, Oak Ridge, TN 37831; prices available from (423) 576-8401.

Available to the public from the U.S. Department of Commerce, Technology Administration, National Technical Information Service, Springfield, VA 22161. 


\section{DISCLAMMER}

Portions of this document may be illegible in electronic image products. Images are produced from the best available original document. 


\section{Contents}

\section{PREFACE}

DIVISION Of CHEMICAL SCIENCES PROGRAM SUMMARIES

\section{LABORATORY ADMINISTRATION}

\section{NATIONAL LABORATORIES}

Photochemical and Radiation Sciences Ames Laboratory

Argonne National Laboratory

Brookhaven National Laboratory

Lawrence Berkeley Laboratory

National Renewable Energy Laboratory

Notre Dame Radiation Laboratory

Chemical Physics

Ames Laboratory

Argonne National Laboratory

Brookhaven National Laboratory

Lawrence Berkeley Laboratory

Lawrence Livermore National Laboratory

Pacific Northwest Laboratory

Sandia National Laboratories, California

Atomic Physics

Argonne National Laboratory

Lawrence Berkeley Laboratory

Lawrence Livermore National Laboratory

Oak Ridge National Laboratory

Chemical Energy

Ames Laboratory

Argonne National Laboratory

Brookhaven National Laboratory

Lawrence Berkeley Laboratory

Los Alamos National Laboratory

National Renewable Energy Laboratory

Oak Ridge National Laboratory

Pacific Northwest Laboratory

Separations and Analysis

Ames Laboratory

Argonne National Laboratory

Brookhaven National Laboratory

Idaho National Engineering Laboratory

Lawrence Berkeley Laboratory

Oak Ridge National Laboratory

Pacific Northwest Laboratory

Heavy Element Chemistry

Argonne National Laboratory

Lawrence Berkeley Laboratory

Los Alamos National Laboratory

Oak Ridge National Laboratory

Chemical Engineering Sciences

Lawrence Berkeley Laboratory

Los Alamos National Laboratory

National Institute for Petroleum and Energy Research

Sandia National Laboratories, California

Advanced Batteries

Ames Laboratory

Argonne National Laboratory

Brookhaven National Laboratory

Lawrence Berkeley Laboratory vii

viii

ix

xiii
Los Alamos National Laboratory

National Renewable Energy Laboratory

Oak Ridge National Laboratory

Sandia National Laboratories, New Mexico

OFFSITE INSTITUTIONS

Photochemical and Radiation Sciences

University of Akron

University of Alabama

Arizona State University

Boston University

Brandeis University

California Institute of Technology

University of California, Berkeley

Colorado State University

University of Colorado

Columbia University

Dartmouth College

Georgia Institute of Technology

University of Houston

Marquette University

University of Massachusetts-Boston

University of Minnesota

National Institute of Standards and Technology, Gaithersburg

University of New Orleans

University of North Carolina at Chapel Hill

Northwestern University

Ohio State University

University of Oregon

Pennsylvania State University, University

University of Pennsylvania

University of Pittsburgh

Portland State University

Princeton University

University of Rochester

Rutgers, The State University of New Jersey

Stanford University

University of Tennessee at Knoxville

University of Texas at Arlington

University of Texas at Austin

Tulane University

Washington State University

University of Washington

Wayne State University

Wichita State University

Chemical Physics

Aerospace Corporation

University of Akron

Arizona State University

University of Arizona

University of California, Los Angeles

University of California, Santa Barbara

Catholic University of America

University of Chicago

University of Colorado

Columbia University

Cornell University 
Emory University

Florida State University

University of Georgia

Harvard University

University of Illinois at Chicago

University of Mlinois at

Urbana-Champaign

Johns Hopkins University

Kansas State University

University of Kentucky

Massachusetts Institute of Technology

University of Massachusetts at Amherst

University of Michigan

University of Minnesota

National Institute of Standards and Technology, Gaithersburg

University of New Orleans

New York University

University of North Carolina at Chapel Hill

North Dakota State University

University of Oregon

Pennsylvania State University, University Park

University of Pennsylvania

Princeton University

Rice University

University of Rochester

University of Southern California

Stanford University

State University of New York at Stony Brook

University of Utah

University of Washington

University of Wisconsin at Madison

Atomic Physics

California Institute of Technology

California State University, Fullerton

Clark Atlanta University

University of Colorado

University of Connecticut

Georgia Institute of Technology

Harvard University

Kansas State University

University of Kansas

University of Kentucky

University of Louisville

Michigan Technological University

University of Nebraska at Lincoln

University of Nevada at Reno

University of New Mexico

City College of New York

University of Notre Dame

Pennsylvania State University, Lehman

Rice University

University of Southern California

University of Tennessee at Knoxville

University of Texas at Austin

University of Toledo

Tulane University

Vanderbilt University

University of Virginia

Western Michigan University

The College of William and Mary

Chemical Energy

University of Arizona

Boston College

California Institute of Technology
University of California, Davis

University of California, Irvine

University of California, Riverside

University of California, Santa Barbara

Carnegie-Mellon University

Colorado State University

University of Colorado

Columbia University

University of Connecticut

University of Delaware

Harvard University

University of Illinois at Urbana-Champaign

Indiana University

University of Iowa

Kansas State University

Lehigh University

Louisiana State University

University of Louisville

University of Maryland at College Park

Massachusetts Institute of Technology

University of Massachusetts at Amherst

University of Memphis

University of Michigan

University of Minnesota

University of Missouri at Columbia

University of North Carolina at Chapel Hill

Northwestern University

University of Oklahoma

Pennsylvania State University, University Park

University of Pennsylvania

University of Pittsburgh

Purdue University

Rensselaer Polytechnic Institute

University of Rochester

Rutgers, The State University of New Jersey

University of South Carolina

University of Southern California

Stanford University

State University of New York at Binghamton

State University of New York at Buffalo

Syracuse University

Texas A \& M University

University of Texas at Austin

Tulane University

University of Utah

Virginia Polytechnic Institute and State University

University of Virginia

University of Washington

University of Wisconsin at Madison

University of Wisconsin at Milwaukee

Yale University

Separations and Analysis

University of Arizona

Auburn University

Brigham Young University

Brown University

Colorado School of Mines

Colorado State University

University of Delaware

Duke University

University of Florida

The George Washington University

iv Research in Chemical Sciences 
Hampton University

University of Illinois at Urbana-Champaign

Kansas State University

Lehigh University

Louisiana State University

Michigan State University

University of Missouri at Rolla

National Institute of Standards and Technology, Gaithersburg

University of North Carolina at Chapel Hill

Purdue University

Rensselaer Polytechnic Institute

State University of New York at Buffalo

Syracuse University

University of Tennessee at Knoxville

Texas A \& M University

Texas Tech University

University of Texas at Austin

University of Utah

Virginia Commonwealth University

Washington State University

University of Wyoming

Heavy Element Chemistry

Florida State University

University of New Mexico

Ohio State University

Chemical Engineering Sciences

University of California, San Diego

Colorado School of Mines

Colorado State University

Cornell University

University of Delaware

University of Illinois at Chicago

Johns Hopkins University

University of Maryland at College Park

University of Massachusetts at Amherst

National Institute of Standards and Technology, Boulder
North Carolina State University

114

University of Pennsylvania

Princeton University

115

Stanford University

115

State University of New York at Stony

116 Brook

University of Tennessee at Knoxville $\quad 116$

Yale University

Advanced Batteries

Arizona State University

116

California Institute of Technology

116

Clark University

University of Minnesota

Moltech Corporation

University of Nevada at Las Vegas

North Carolina State University

Pennsylvania State University, University Park

Rutgers, The State University of New Jersey

Texas A \& M University

Small Business Innovation Research

ANDCARE, Inc.

Analysis Consultants

Compact Membrane Systems, Inc

Consultec Scientific, Inc.

EIC Laboratories, Inc.

Materials and Electrochemical Research Corp.

Membrane Technology \& Research, Inc.

Microsensor Systems, Inc.

TDA Research, Inc. 



\section{PREFACE}

This summary book is published annually to provide information on research supported by the Department of Energy's Division of Chemical Sciences, which is one of four Divisions of the Office of Basic Energy Sciences in the Office of Energy Research.

These summaries provide the scientific and technical public, as well as the legislative and executive branches of the Government, information, either generally or in some depth, about the Chemical Sciences program. Areas of research supported are indicated in the section heading, the "Selected Topics of General Interest" list, and the summaries themselves. Energy technologies that may be advanced by use of the basic knowledge generated in this program are included in the "Selected Topics of General Interest" list and are often referenced in the summaries.

Scientists interested in proposing research for support will find the publication useful for gauging the scope of the present basic research program and it's relationship to their interests. Proposals that expand this scope may also be considered or directed to more appropriate offices. The primary goal of the research summarized here is to add significantly to the knowledge base in which existing and future efficient and safe energy technologies can evolve. As a result, scientific excellence is a major criterion applied in the selection of research supported by the Division of Chemical Sciences, but another important consideration is emphasis on science that is advancing in ways that will produce new information related to energy.
The program takes place in several different kinds of performing organizations. About one-third of the projects are at DOE laboratories and the rest mostly at universities, with a small number of projects at industrial laboratories. In DOE laboratories, much of the research utilizes special unique facilities which, in some instances, are national user facilities. These are described in a special section of this publication.

Another section of this publication includes summaries of projects funded during FY 1995 by the Small Business Innovation Research (SBIR) Program Office in response to technical topics submitted by the Division of Chemical Sciences to the annual SBIR Solicitation and the annual Small Business Technology Transfer Solicitation.

Questions about the detail of an individual project may be directed to the investigators involved or the persons in charge at $\mathrm{DOE}$ laboratories (who are identified at appropriate places in this publication). Other questions about the program may be directed to the undersigned.

Robert S. Marianelli, Director

Division of Chemical Sciences Office of Basic Energy Sciences 


\section{Division of Chemical Sciences Organizational Chart}

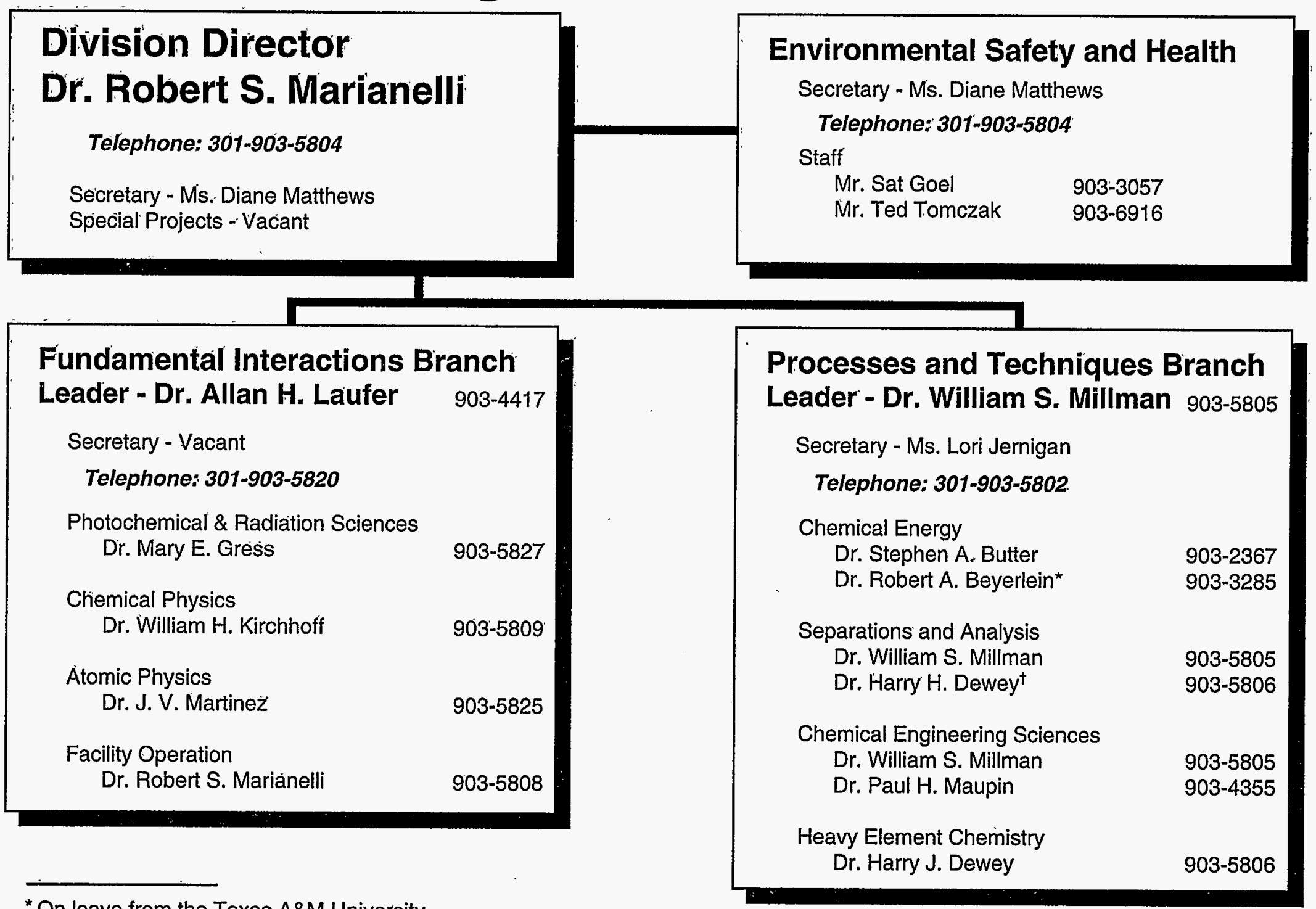

* On leave from the Texas A\&M University

${ }^{\dagger}$ On leave from the Los Alamos National Laboratory

Chemical Sciences Division Office of Energy Research U.S. Department of Energy Germantown, MD 20874-1290 


\section{PROGRAM SUMMMARIES}

Each Branch of the Division of Chemical Sciences is divided into programs that cover various disciplines. The following summaries describe these programs. The staff members responsible for each program are indicated on page viii. The Division has the responsibility for seeing that the research programs and facilities it supports are operated in a safe manner with due regard for the health of participants and the protection of the environment. The staff who do this for the Division and the Office of Basic Energy Sciences are in the Division of Chemical Sciences and the Materials Sciences Division. The Chemical Sciences Environment Safety and Health (ES\&H) staff are also indicated on page viii.

\section{Fundamental Interactions Branch (KC-03-01)}

\section{Photochemical and Radiation Sciences (KC-03-01-01)}

The Photochemical and Radiation Sciences program consists of research on the interactions of radiation with matter. Emphasis is placed on exploration of fundamental photochemical processes aimed at the capture and conversion of solar energy. The solar photochemistry research encompasses organic and inorganic photochemistry, electron and energy transfer in homogeneous and heterogeneous media; photocatalysis, and photoelectrochemistry. The photosynthetic reaction center is studied as a model for design of efficient photoinduced charge separation in biomimetic/photocatalytic assemblies. The radiation chemistry research is concerned with the chemical effects produced by absorption of energy from ionizing radiation. Electron pulse radiolysis techniques provide information on the nature of transient intermediates, kinetics, and mechanisms of chemical reactions in the condensed phase.

\section{Chemical Physics (KC-03-01-02)}

The Chemical Physics program supports research on fundamental molecular processes related to the mission of the Department in the areas of combustion, catalysis, and environmental restoration. Specific areas of emphasis include gas phase chemical reaction theory, experimental dynamics and spectroscopy, thermodynamics of reaction intermediates, chemical kinetics and reaction mechanisms at high temperatures in the gas phase and at surfaces, combustion diagnostics, and chemical dynamics and kinetics at surfaces and with metal and semiconductor clusters.

A major user-oriented facility, the Combustion Research Facility at Sandia.National Laboratories, California, is supported by this program. This facility offers the use of advanced instrumentation and computation to interested combustion scientists from industry, universities, and national laboratories.

\section{Atomic Physics (KC-03-01-03)}

The Atomic Physics program supports experimental and theoretical studies of atom and atomic ion structures, energy levels and lifetimes of quantum states, and of transport and exchange processes associated with energy and momentum transfer. These studies seek to obtain the most accurate and complete fundamental knowledge of the properties and interactions of photons, electrons, atoms, and ions. Relatively high energy atomic physics research involving atoms stripped of all or almost all electrons and of atoms and ions in which electrons are promoted to upper energy levels provide basic information to assist thermonuclear energy development. The study of processes that lead to the production of coherent radiation and its statistical manifestations in atomic physics are included in this program to assist development of other less well characterized energy technologies. Research on the manipulation of atoms with coherent photon fields, on the behavior of plasmas generated by intense laser beams, and on the physics of low-temperature plasmas relevant to materials processing is also supported.

\section{Facility Operations (KC-03-01-04)}

DOE operates large scientific facilities for the benefit of the scientific community. Major user facilities permit forefront research to be conducted in areas important to DOE by scientists from industry and universities in addition to DOE contractors/grantees. Operating support for these expensive, unique facilities that are open to all qualified researchers is provided by DOE. Many of the facilities are user oriented. The four operated by Chemical Sciences are the following: the Combustion Research Facility (CRF) at Sandia, Livermore, the High Flux Isotope Reactor (HFIR) at Oak Ridge National Laboratory, the Stanford Synchrotron Radiation Laboratory (SSRL) at Stanford; and, shared with the Materials Sciences Division, the 
National Synchrotron Light Source (NSLS) at Brookhaven. They represent research resources for the general scientific community, and qualified scientists not associated with the host laboratory are encouraged to make use of them. However, any activity that can be carried out at commercially available laboratories is not appropriate for these DOE-supported facilities. The process by which an off-site scientist can use a facility is discussed in each summary.

In addition, another facility at Oak Ridge is operated as a service to the scientific community: the Radiochemical Engineering Development Center (REDC).

Other facilities described in the "Special Facilities" section are also available for use through collaboration with staff scientists. The names of individuals to contact for further information and technical data on available instrumentation at each facility are described.

\section{Processes and Techniques Branch (KC-03-02)}

\section{Chemical Energy (KC-03-02-01)}

This program includes basic chemistry research related to chemical transformations or conversions which are fundamental to new or existing concepts of energy production and storage. Of particular interest are those research activities with the objectives of understanding the chemical aspects of (1) catalysis, both heterogeneous and homogeneous; (2) the chemistry of fossil resources, particularly coal, including characterization and transformation; (3) the conversion of biomass and related cellulosic wastes; and (4) the chemistry of precursors to advanced materials. The disciplines of organic, organometallic, inorganic, physical, thermo-, and electrochemistry are central to these programs. The emphasis is on understanding the fundamental chemical principles underlying the new and developing technologies and on innovative chemical research with potential for new energy concepts.

\section{Separations and Analysis (KC-03-02-02)}

The separations part of the program supports basic research directed toward improving our understanding of methods for separating mixtures of gases, liquids, solids, and their component molecules, cations, and anions. The program covers a broad spectrum of separations concepts, including membrane processes, extraction under both standard and supercritical conditions, adsorption, chromatography, photodissociation, complexation, etc. The research involves elucidating fundamental chemical phenomena for improved or new separations rather than developing specific processes.

The analysis part of the program supports research on phenomena basic to analytical methods, the goal being to improve sensitivity, reliability, and/or productivity of analytical determinations. Chemical and physical principles which can lead to entirely new methods of analysis are investigated, although this program does not support instrument development. Research progress is reported quickly in the open literature so that those interested in instrument development can build on work supported herein. The program is aimed at obtaining a thorough understanding of the basic chemistry of analytical techniques so that their utility can be improved, rather than solving specific problems in analysis.

\section{Heavy Element Chemistry (KC-03-02-03)}

The Heavy Element Chemistry program focuses on a study of the chemical and certain physical properties of the actinide elements, principally the transuranium elements, because of the importance of these elements to DOE's nuclear technology programs and to an understanding of the basic science in general. A variety of investigations are pursued, including (1) organometallic chemistry, (2) the chemistry of excited spectroscopic states, (3) thermochemistry of actinide elements and compounds, (4) the chemistry of actinides in near-neutral aqueous solutions and the reactions of aqueous actinide ions with various complexing agents, (5) the development of preparative methods for actinide metals and compounds, and (6) characterization of actinides in the solid state under pressure. This research is performed principally at the national laboratories because of facilities required for handling radioactivity.

\section{Chemical Engineering Sciences (KC-03-02-04)}

This program addresses energy aspects of chemically related engineering topics, including thermodynamics, turbulence related to combustion, and physical and chemical rate processes. Particular attention is given to experimental and theoretical aspects of phase equilibria, especially of mixtures, including supercritical phenomena, and to the physics of gas phase turbulence. Also included are fundamental studies of thermophysical and thermochemical properties. Emphasis is given to improving and/or developing the scientific base for engineering generalizations and their unifying theories. 


\section{Advanced Battery Technology (KC-03-02-04)}

The Advanced Battery Technology Research and Development program supports research to develop new generic battery technology focused on the nonautomotive consumer market with emphasis on improvements in battery size, weight, life, and recharge cycles. Areas of research include materials development and characterization, battery component development and interactions, characterization methodologies, and systems development and modeling. Although both primary and secondary battery systems are considered, the greatest emphasis is placed on rechargeable (i.e., secondary) battery systems. The program covers a broad spectrum of research including investigations of lithium cells, metal hydrides, bifunctional air electrodes, fundamental studies of composite electrode structures, failure and degradation of active electrode materials, and thin-film electrodes, electrolytes, and interfaces. Characterization and methodologies include problems of electrode morphology, zinc corrosion, separator/electrolyte stability, stable microelectrodes, and the transport properties of electrode and electrolyte materials and surface films. Investigations in computational chemistry, modeling, and simulations, including property predictions, phenomenological studies of reactions and interactions at critical interfaces, film formation, phase change effects on electrodes and characterization of crystalline and amorphous materials are also of interest. 


\section{LABORATORY ADMINISTRATION}

Listed below, by laboratory and department or division, are persons who are or will be in charge of the Division of Chemical Sciences projects at DOE laboratories. These individuals are laboratory, department, or division administrators who can provide information about specific programs or refer inquiries to appropriate individuals.

\section{AMES LABORATORY}

Iowa State University

Ames, IA 50011

\section{Chemical Sciences-Fundamental Interactions}

A. DePristo

Phone: (515) 294-9924

Chemical Sciences-Processes and

Techniques

J. H. Espenson

Phone: (515) 294-5730

\section{Science and Technology}

R. B. Thompson

Phone: (515) 294-9649

\section{ARGONNE NATIONAL LABORATORY}

9700 South Cass Avenue

Argonne, IL 60439

\section{Chemical Technology Division}

J. E. Battles

Phone: (708) 252-4383

Chemistry Division

M. C. Thurnauer

Phone: (708) 252-3570

\section{Physics Division}

L. Young

Phone: (708) 252-4039

\section{BROOKHAVEN NATIONAL LABORATORŸ}

P.O. Box 5000

Upton, NY 11973-5000

\section{Department of Applied Science}

J. Davenport

Phone: (516) 282-3037
Chemistry Department

C. Creutż

Phone: (516) 282-4301

National Synchrotron Light Source

M. Hart

Phone: (516) 282-4966

IDAHO NATIONAL ENGINEERING

LABORÄTORY

EG\&G Idaho, Inc.,

P.O. Box 1625

Idahóo Falls, ID 83415

Chemical and Materials Pròcessè

Department

D. L̀. Míller

Phone: (208) 526 $=9052$

\section{LAẄRENCE BERKELEY LABORATORY}

Üniversity of Califorinia

Berkeley, CA 94720

Structựal Biónogỳ Divisision

S. H. Kim

Phone: (5்10) 486-435̃

Chemical Sciènces Division

C. Härris

Phone: (510) 486-6382

- Energy and Ėnvirononimentit Division

E. J. Cairns

Phone: (510) 486 6 -5020

Nuclear Science Divivision

T. J. M. Symonis

Phone: (510) $486=5670$

\section{LAWRËNCE LIVERTOTORE NATIÓNAL}

LABOORATTORYั

Ưniviversitỹ of Califoriñia

P.O. Box 808

Liverrmore, CA 94550

Phhysics añd Sipace Techinòiógy

Direectoriàte

D. Schneider

Phone: (5 10$)$ 423-5940 
LOS ALAMOS NATIONAL LABORATORY

University of California

P.O. Box 1663

Los Alamos, NM 87545

Energy Technology Programs Office

C. J. Burns

Phone: (505) 667-3880

\section{NATIONAL RENEWABLE ENERGY}

\section{LABORATORY}

1617 Cole Boulevard

Golden, CO 80401

Basic Sciences Division

S. Deb

Phone: (303) 384-1105

NOTRE DAME RADIATION LABORATORY

University of Notre Dame

Notre Dame, IN 46556

K.-D. Asmus

Phone: (212) 631-5561

\section{OAK RIDGE NATIONAL LABORATORY,}

P.O. Box 2008

Oak Ridge, TN 37831

Chemical Technology Division

L. E. McNeese

Phone: (423) 574-6275

Chemical and Analytical Sciences Division

M. L. Poutsma

Phone: (423) 574-5028

Physics Division

S. Datz

Phone: (423) 574-4984
PACIFIC NORTHWEST LABORATORY

P.O. Box 999

Richland, WA 99352

Environmental and Energy Sciences

Division

B. R. Stultz

Phone: (509) 375-2687

Environmental and Molecular Sciences

Laboratory

T. H. Dunning

Phone: (509) 375-6863

\section{SANDIA NATIONAL LABORATORIES,} NEW MEXICO

P.O. Box 5800

Albuquerque, NM 87185

Nanostructures and Advanced Materials Research Department

G. Samara

Phone: (505) 844-6653

\section{SANDIA NATIONAL LABORATORIES,} CALIFORNIA

P.O. Box 969

Livermore, CA 94551

Combustion Research Facility

G. A. Fisk

Phone: (510) 294-3376 


\section{Photochemical and Radiation Sciences}

\author{
Ames Laboratory \\ Iowa State University \\ Ames, IA 50011
}

\section{Fundamental Interactions Program}

\section{Raman Spectroscopy of Model \\ Photosynthetic Systems \\ Cotton, T.M.; Chumanov, G. 515-294-9887}

$\$ 150,000$

The objective of this project is to study heterogeneous electron transfer between different molecular assemblies at metal surfaces with the ultimate goal to prepare model photosynthetic systems. Monolayers and multilayers composed of donor acceptor species are fabricated by selfassembly and Langmuir-Blodgett techniques. This approach allows the control of structure and composition of these molecular assemblies. The distinguishing feature of this project is the use of metal substrates as a means of enhancing different optical phenomena as well as photoinduced electron transfer. Such enhancement occurs due to excitation of the plasmon resonances on specially prepared metal surfaces. Recently, a completely new concept was developed based on the coupling of the plasmon resonances of individual metal particles in highly organized two-dimensional arrays. The coupling produces nonradiative energy transfer between particles resulting in highly efficient light collection. Such substrates (termed Colloidal Metal Films or CMFs) were successfully prepared using surface chemistry to immobilize small silver and gold particles on glass surfaces. Currently, their properties are under investigation with the goal of developing an artificial light antenna system. In the future, this artificial antenna will be coupled to different model photosynthetic systems in order to increase significantly their efficiency.

\section{Laser Spectroscopy and Electronic Energy Transfer of Light Conversion Systems Small, G.J.; Hayes, J.M.; Reddy, N.R.S. 515-294-3859 $\$ 235,000$}

Electronic energy and electron transfer processes in photosynthetic protein complexes are investigated in order to understand the structure-dynamics relationships that lead to the high solar energy conversion efficiency of photosynthesis. Attention is paid to characterizing the excited electronic states of chlorophylls and other cofactors that are bound to the protein, understanding how lowfrequency protein vibrations mediate the transport of energy or charge, and understanding the effect of the inherent structural disorder of proteins on the kinetics of transport. High-resolution laser-based frequency domain spectroscopies (e.g., spectral hole burning) developed in this laboratory are applied. Currently, hole burning is the most powerful method for the study of the above problems and has provided the first quantitative data on electronvibration (protein) coupling and the glass-like disorder of proteins. These data are used to test existing theoretical models and to develop new ones. Because the understanding of energy/electron transport and solar energy conversion is complex, new experimental approaches are developed. Recently, a novel high pressure/variable temperature apparatus has been interfaced with the laser-spectroscopic system and used to demonstrate that the pressure dependence of kinetics provides new insights on electron and energy transport mechanisms.

\section{Ultrafast Spectroscopy and Energy Transfer \\ Struve, W.S.; Savikhin, S. 515-294-4276}

$\$ 290,000$

Ultrafast laser spectroscopy is used to study electronic excitation transport, vibrational equilibration, and charge separation on the femtosecond time scale in natural and artificial photosynthetic systems. Recent areas of emphasis have included comparisons of energy transfer kinetics in oligomeric bacteriochlorophyll (BChl) light-harvesting antennae in green bacteria with those in their reconstituted BChl aggregates; studies of excitation annihilation in very large antennae in the limit of very low laser intensities, with a view to determining their functional antenna size; modeling the role of vibrational cooling in the pump-probe kinetics of BChl-containing pigment-protein complexes; and studying the effects of quantum interferences from exciton couplings on the absorption difference sepctroscopy of strongly coupled antennae. In a biomimetic project pursued cooperatively with other investigators in the Ames Laboratory, two types of synthetic lightharvesting antennae are being prepared and investigated: artificial pigment-protein complexes consisting of phthalocyanine chromophores mounted on modified (VPGKG) elastin helices; and Coulomb tetraazaphthalocyaninephospholipid bilayers in Langmuir-Blodgett films.

\section{Argonne National Laboratory Argonne, IL 60439}

\section{Chemistry Division}

\section{Electron Transfer and Energy Conversion Miller, J.R.; Meisel, D.; $\quad \$ 1,097,000$} Curtiss, L.A. 708-252-3481

This project involves fundamental research on intramolecular electron transfer and charge transfer at interfaces in microheterogeneous environments. Recent efforts concern: (a) exploration of how electron transfer between molecules is controlled by distance, energy, and molecular structure; (b) electron transfer at surfaces of semiconductor nanoparticles and between such particles; (c) the nature and dynamical characteristics of excited states of radical 
ions and free radicals. Experimental methods to couple high-speed lasers to an electron linear accelerator are under development for the study of radical species and for the possible extension to light-driven electron transfer reactions of these species. The pulsed electron radiolysis technique is uniquely suited to investigate the role of important variables such as distance, energy, ion pairing, and solvent polarity on electron transfer, because it enables experiments which clearly delineate the role of each variable. These capabilities are augmented in this program by coupling to theory at the ab initio level.

\section{Photosynthesis Research Norris, J.R:; Thurnauer, M.C.; Tiede, D.M.; Wasielewski, M.R.; Tang, J:; Chen, L.X. 708-252-3544}

This research is devoted to advancing the understanding of natural photosynthesis for application in artificial photosynthesis. This work explores the detailed mechanics of how an electron is pumped by light activation from the primary donor (bacteriochlorophyll special pair) to the primary acceptor (bacteriopheophytin). Why this natural chemistry appears to require 10 times less work by the surrounding medium than in artificial photosynthesis is explored by femtosecond optical spectroscopy in genetically modified bacterial reaction centers. Another major goal of this work is to establish the characteristics of the quinone photochemistry that results in long-lived charge separation. During the quinone activity, pairs of free radicals are created that can be probed by unique state-ofthe-art time domain magnetic resonance techniques. Isotopically labeled material is required for these magnetic resonance experiments. Finally, a theoretical component is devoted to studying the primary charge separation events and to modeling the state-of-the-art electron paramagnetic resonance characterization of charge separation across a storage barrier.

\section{Preparation and Photophysical Characterization of Photosynthetic Components}

Norris, J.R.; Tiede, D.M.

708-252-3547

$\$ 100,000$

The purpose of this work-is to provide the necessary compounds, reagents, and components for unraveling the chemical machinery of natural and artificial photosynthesis. Many experiments designed to advance the understanding of the initial, solar-energy-conversion chemistry of natural and artificial photosynthesis. require special reagents, especially reagents that contain nonstandard, stable isotopes. In particular, materials labeled with stable isotopes are prepared and their physical and chemical properties are fully characterized. For example, fully deuterated reaction center proteins and fully deuterated chlorophylls and bacteriochlorophylls are biosynthesized, purified, and characterized by optical and magnetic resonance spectroscopies and mass spectrometry. These fully deuterated materials are routinely required to track the chemical events of natural and artificial photosynthesis by state-of-the-art spectroscopic techniques. Also, fully deuterated material, including the reaction center protein, is necessary to probe the microscopic structure of the photosynthetic machinery with neutron scattering and diffraction. Finally, other fully characterized, isotopically labeled material containing carbon-13 and/or nitrogen-15 are available as required for magnetic.resonance spectroscopies, and are invaluable analytical tools for probing the components, structure, and mechanisms of charge separation as performed by natural and artificial photosynthetic systems.

\section{Reactive Intermediates in the Condensed Phase: Radiation and Photochemistry Trifunac, A.D.; Sauer, M.C., Jr.; $\quad \$ \mathbf{1 , 8 2 9 , 0 0 0}$ Bartels, D.M.; Werst, D.W.; \\ Jonah, C.D. 708-252-3483:}

This research program encompasses fundamental studies of chemical effects of ionizing radiation. The purpose is to develop detailed knowledge of chemical consequences of the deposition of energy in condensed matter by energetic particles and photons. The knowledge developed in the course of this research will benefit diverse areas of technology, such as radioactive waste management, polymer processing, food sterilization, and treatment of hazardous wastes. The real-time capabilities used are at the leading edge of studies of ultrafast chemistry induced by ionizing radiation. The state-of-the-art time-domain tools include a picosecond linear accelerator, a Van de Graaff accelerator, and subpicosecond and nanosecond UV lasers coupled to specialized detection equipment. This array of capabilities allows a comprehensive analysis of the complex chemistry and transient intermediates induced by ionizing radiation. The fundamental processes of charge transport and ion-molecule reactions and the discovery of new transients and their chemistry are described. The role of solvent in controlling the reactivity of charged and neutral species is analyzed experimentally and theoretically. New insights and probes of solvent structure are developed. The study of transient species in solid-state systems allows control of the chemistry via matrix-ion interaction.

\section{Artificial Photosynthesis Wasielewski, M.R:; Wiederrecht, G.P. 708-252-3538}

$\$ 719,000$

The principal goal of the artificial photosynthesis project is to design, prepare, and study the fundamental properties of:molecular systems that will efficiently convert light energy into useful chemical energy. The picosecond, high quantum yield photochemical charge separation that occurs in natural photosynthesis serves as a conceptual model for the systems studied in this project. Artificial photosynthetic charge separation systems are designed to yield photocatalysts that will perform well in practical chemical environments. New supramolecular systems that consist of covalently linked arrays of electron donors and acceptors have been synthesized. These systems utilize visible light to separate charge with $80 \%$ efficiency and separation lifetimes that exceed 1 millisecond. The organic electron donors and acceptors within these supermolecules maintain well-defined structural, solvation, and electronic relationships among themselves. Current research focuses on issues that are fundamental to optimizing charge separation and storage efficiencies in the solid state. This includes the interplay between the properties of the organized donor-acceptor array and the 
molecular organization of the surrounding medium. Ultrafast laser spectroscopy is used to probe the molecular interactions that occur in these ordered systems.

\section{Brookhaven National Laboratory Upton, L.I., NY 11973}

\section{Department of Applied Science}

\section{Porphyrin Chemistry \\ Fajer, J.; Barkigia, K.M.; Renner, $M$.W. $516-282-4521$}

This program addresses the role of porphyrin derivatives in bioenergetic reactions with particular emphasis on the mechanisms by which light is harvested and converted into chemical energy in photosynthesis. The cumulative body of structural, theoretical, and experimental data evolving from the photoconversion modelling is equally applicable to the multielectron catalyses of nitrogen assimilation, regiospecific reactions, and carbon dioxide conversion to methane mediated by the broad class of porphyrins. To model these photochemical and catalytic reactions, the project uses photochemistry, spectroelectrochemistry, magnetic resonance, X-ray, and synchrotron radiation techniques that are closely supported by theoretical methods. In particular, readily modified synthetic and theoretical models offer useful insights into reactions that occur on picosecond time scales. Recent results for such models have provided clear evidence of the significant role that structural variations play in controlling optical, redox, and excited state properties as well as sites of reactions in vitro and in vivo. The cumulative thrust of the combined theoretical, structural, and experimental approaches is to characterize transients and mechanisms in bioenergetic conversion, and to provide specific guidelines for the development of synthetic photocatalytic systems.

\section{Electrochemistry and Photoelectrochemistry Feldberg, S.W.; Smalley, J.F. 516-282-4480}

The objective of this program is to provide new insights into the mechanisms of electrochemical and photoelectrochemical phenomena. The experimental objective is to understand the role of interfacial structure and organization in a variety of interfacial processes [e.g., double-layer relaxations, charge (ion or electron) transfer between the solution and the electrode, electron transfer between electrode and immobilized or adsorbed redox moieties]. The focus is on heterogeneous electron transfer and, in particular, on the measurement of very fast (submicrosecond) heterogeneous processes. The experimental approach utilizes an indirect laser-induced interfacial temperaturejump (ILIT) technique. At its present level of development, IIIT can access interfacial rate processes occurring in the nanosecond time domain, arguably a leading-edge experimental approach exceeding the capabilities of other state-of-the-art electrochemical approaches. Theoretical analyses and computer simulations attack a broad range of electrochemical and photoelectrochemical problems that may be generally described as electron transfer coupled with homogeneous chemical reactions.
Chemistry Department

\section{Radiation Chemistry Schwarz, H.A.; Holroyd, R.A.;Cabelli, D. E.; Wishart, J.F. 516-282-4330}

\section{$\$ 1,010,000$}

This program uses pulse radiolysis, photolysis, and synchrotron Xrays to study the behavior of transient chemical species. Principal areas of investigation are: (1) pressure effects on the properties and reactions of electrons and ions in dielectric fluids; (2) X-ray track effects in dielectric fluids; (3) formation and reactions of transition-metal complexes in unusual oxidation states; (4) the dependence of electron-transfer rates on distance, driving force, and reorganization energy; and (5) the nature, thermodynamics, and reactions of radicals produced in aqueous solutions. The studies of electrons in dielectric fluids are of prime importance in understanding the basic processes of radiation chemistry, and in the development of new detectors for high-energy physics. The studies of transition-metal complexes are important in developing and understanding certain homogeneous catalysis systems and in schemes for chemical storage of solar energy. Knowledge of the thermodynamics of radicals is essential in understanding their electron-transfer reactions. The goal of the electron-transfer studies is to gain understanding of long-range effects, which can be applied to the capture and storage of solar energy.

\section{Thermal and Photoinduced Reactions in Solution \\ Sutin, N.; Creutz, C.; \\ Newton, M.D.; Seltzer, S.; \\ Brunschwig, B.S.; Fujita, E.; \\ Castner, E.W. \\ 516-282-4358 \\ $\$ 1,875,000$}

This program addresses three areas fundamental to the efficient capture and storage of light energy: excited-state formation, chemistry, and photophysics; energy transduction by electron-transfer reactions; and energy storage through chemical transformations. Theoretical and experimental efforts are elucidating the factors controlling excited-state lifetimes and electron-transfer rates; the roles of electronic configuration, donor/acceptor separation, bridging groups, nuclear-configuration and free-energy changes, as well as the effects of solvent dynamics are being investigated through studies of transition-metal complexes and other donor/acceptor systems. Dipole moment changes in charge-transfer transitions are being probed through Stark spectroscopy, and the charge-transfer photochemistry of dinuclear systems is being studied by fiash-photolysis techniques. The longterm storage of solar energy as fuels or valuable chemicals requires efficient coupling of light-absorption and chemical transformation processes. Mechanistic studies of the photoinduced and thermal olefin cis-trans isomerization address the nature of this coupling in the purple membrane proton pump, a solar energy converter that operates through photoinduced and protein-catalyzed cis-trans isomerization of its bound retinal. Chemical and mechanistic studies of transition-metal centers in high and low oxidation states are being conducted because such centers can couple photoinduced electron-transfer processes to the 
bond-forming reactions required in the photodecomposition of water or the photoreduction of carbon dioxide to useful chemicals.

\section{Lawrence Berkeley Laboratory University of California, Berkeley Berkeley, CA 94720}

\section{Structural Biology Division}

\section{Artificial Photosynthesis Calvin, M.; Otvos, J.W. 510-643-5237}

The project objective is to devise a synthetic system for storing the energy of visible light. The approach involves basic photochemical research in areas that will improve understanding of the relevant parts of such a storage system. First, there is a photoinduced electron-transfer process across a phase boundary, mimicking the natural photosynthetic process, followed by a stabilization of the initial electron-transfer products. These products are then catalytically converted to hydrogen and oxygen, the results of water photolysis, or to reduced organic compounds and oxygen if carbon dioxide is used as the substrate. The catalytic conversion of the initial oxidized product to oxygen is the most difficult challenge. Over the past several years considerable progress has been made in synthesizing and characterizing oligomers of transition metals complexed with macrocyclic ligands. This type of compound, which can exist in several oxidation states, is an attractive candidate for catalyzing the multi-electron oxidation of water. The project is focusing on dinuclear complexes of iron and ruthenium with a novel conjugated ligand coordinating nitrogen atoms.

\section{Chemistry with Near Infrared Photons Frei, H.M. 510-486-4325 \\ $\$ 315,000$}

The objective of this project is to establish useful chemistry that can be accomplished with the sun's most abundant, long-wavelength photons. Current focus is on reactions that suggest new concepts in red and nearinfrared light-assisted synthesis of organic building blocks, high-value compounds, and fuels from abundant chemicals. Controlled atom and group transfer reactions are explored by excitation of reactant pairs in inert cryogenic matrices. Most recent examples are completely regio and stereospecific photooxidations of small alkenes and alkynes by red light-excited nitrogen dioxide. The unique photochemistry of cage reactant pairs is being explored for new controlled synthesis in zeolite matrices at ambient temperature. Highly selective oxidation of small alkenes, alkanes, and substituted aromatics by molecular oxygen has been achieved with visible light at room temperature. Examples include conversion of toluene to benzaldehyde, propylene to acrolein, and cyclohexane to cyclohexanone. Laser photochemical and in situ Fourier transform infrared probing methods are employed to elucidate reaction paths. This is the first selective method for hydrocarbon oxidation by oxygen at high conversion. A central aspect of the new method is the exploitation of the very high electrostatic field inside the zeolite cage. It stabilizes the excited hydrocarbon-oxygen charge-transfer state by 1.52.5 electron volt, thus allowing initiation of the reaction with low-energy visible photons.

\section{National Renewable Energy Laboratory Golden, CO 80401}

\section{Basic Sciences Division}

\section{Surface-Modified Semiconductor Electrodes and Novel Electrolyte-Semiconductor Interfaces Frank, A.J. 303-384-6262

This research addresses basic issues in photoelectrochemistry related to advancing the understanding of charge transfer processes at chemically-modified photoelectrodes and conducting polymers, improving the efficiency and stability of semiconductor electrodes, and developing new electrocatalysts for water oxidation. The conversion efficiency and stability of $\mathrm{p}$-Si electrodes in photoelectrochemical cells were improved by electrochemical modification of the surface. The fundamental nature of charge carriers has been determined in the semiconductive polymer sexithiophene. Solid polymer electrolytes are being studied as potential replacements for liquid electrolytes in photoelectrochemical cells. The water oxidation kinetics of a Ru-based molecular catalyst that is chemically affixed to the surface of an electrode is being investigated.

\section{Photoconversion Processes in Liquid Crystal Porphyrin Films and Other Molecular Semiconductors Gregg, B.A. 303-384-6635}

The photochemical and photophysical properties of thin films of liquid crystal porphyrins and other organic semiconductors are being investigated to understand the relationships between the photoconversion processes in these organic systems and those in inorganic semiconductors, as well as in biological photosynthesis. In molecular photoconverters, charge carriers are produced by dissociation of excitons formed upon light absorption; therefore, understanding exciton transfer and dissociation is important. These fundamental processes are being studied in highly ordered films of liquid crystal porphyrins, perylenes, and phthalocyanines. The mechanisms of interfacial charge generation are being investigated using redox polymers or molecular donors and acceptors as contacts to the organic photoconverters. Another aspect of the project involves the synthesis and characterization of selfoptimizing photochemical systems in which, for example, the quantum efficiency for energy transfer increases with illumination time. This system may provide a realistic model for the light-harvesting system of biological photosynthesis. 
17. Synthesis and Characterization of Novel III-IV and II-VI Semiconductor Quantum Dot Structures for Solar Photochemical Conversion Mícic, O.I. 303-384-6165 $\$ 106,000$

The unique photochemical and photocatalytic properties of quantum dot structures are being investigated for solar photochemical conversion. Very high quality, crystalline colloidal quantum dots of InP, GaP, and $\mathrm{GaInP}_{2}$ with mean diameters ranging from 25 to $70 \AA$ are being synthesized and studied. The synthesis of the III-V colloidal nanocrystallites is achieved by first reacting appropriate chlorometaloxalate complexes with trimethylsilylphosphine to form an organometallic III-V precursor, then heating the precursor using trioctylphosphine oxide as a stabilizer in high boiling solvents $\left(240\right.$ to $400{ }^{\circ} \mathrm{C}$ ) for several days to produce quantum dots that can be dissolved in nonpolar organic solvents to form transparent colloidal quantum dot dispersions. The high sample quality of the InP and $\mathrm{GaP}$ quantum dots results in the first observation of excitonic features in the absorption spectra in these nanocrystals. Fundamental photochemical and photophysical processes in the quantum dots are being examined using ultrafast transient and steady-state spectroscopy in collaboration with other investigators.

\section{Carrier Dynamics and Quantization Effects in Photoelectrochemistry Nozik, A.J.} 303-384-6603

This research is concerned with the theoretical and experimental characterization of the relaxation dynamics of photogenerated carriers (electrons and positive holes) in semiconductor electrode structures in photoelectrochemical and photocatalytic cells. The relevant phenomena include carrier generation, carrier cooling, solid-state carrier transport, and carrier transfer across the semiconductor-liquid interface to redox acceptors or donors at or near the semiconductor surface. The effects of electric fields, as well as the effects of size quantization in semiconductor quantum dots and quantum films, on the carrier dynamics are also being investigated. A detailed understanding of the carrier dynamics is critical for understanding the fundamental behavior of semiconductor-liquid junction structures, and for developing and optimizing their future applications.

\section{Fundamental Studies of Electron Injection Dynamics at Dye-Sensitized Nanostructured Semiconductors in Photochemical Solar Cells Nozik, A.J. 303-384-6603}

$\$ 150,000$

The rates of electron injection from photoexcited dye molecules adsorbed onto the surface of $\mathrm{TiO}_{2}$ nanoparticles into the $\mathrm{TiO}_{2}$ conduction band are being studied by femtosecond time-resolved photoluminescence spectroscopy using the upconversion technique, and by femtosecond transient absorption spectroscopy. $\mathrm{TiO}_{2}$ nanoparticles formed into a highly porous thin film nanostructured electrode is the basis for a newly-developed photochemical solar cell that is reported to exhibit solar conversion efficiencies greater than 10\%; this system represents a new approach to low cost and efficient photovoltaic cells. The quantum yield for charge injection from the excited dye to the $\mathrm{TiO}_{2}$ nanostructured electrode is reported to approach $100 \%$. In this research, the mechanism responsible for this highly unusual quantum efficiency for electron transfer is being investigated, and parameters are being defined that control the quantum yield. These basic studies are supporting efforts to develop the photochemical solar cell into a practical system.

\section{Isolated Photosystem II Reaction Center and Sub-Core Complexes \\ Seibert, $M$. \\ 303-384-6279 \\ $\$ 34,000$}

High-quality, stable preparations of isolated photosystem II reaction centers (RC), the proximal antenna pigment CP47, and RC-CP47 complexes have been prepared using special membrane solubilization agents. In collaboration with other investigators, these important membraneprotein complexes are being investigated by various ultrafast transient spectroscopic techniques in order to establish the kinetics and mechanisms of photoinduced energy transfer and primary charge separation. These studies are important for understanding in detail the mechanisms of oxygen evolution during biological photosynthesis.

\section{Notre Dame Radiation Laboratory University of Notre Dame Notre Dame, IN 46556}

\section{Radiation Laboratory}

\section{Radiation Chemical Studies on Reactive Intermediates \\ Asmus, K.D.; Guldi, D.M. 219-631-5561

Current studies focus on three topics: heteroatomcentered free radicals, functionalized fullerenes, and free radical-induced degradation of halogenated organic acids. Investigations are mainly carried out by pulse radiolysis, complemented by $\gamma$-radiolysis, photolysis and analytical chromatography. The first topic is presently concerned with radical cations, which are characterized by heteroatom-heteroatom three-electron $(2 \sigma) / 1 \sigma^{*}$ bonds. The presence of an antibonding $\sigma^{*}$ electron renders these systems an ideal object to study weak bonding in a thermodynamically stabilized intermediate state in bond breaking and bond formation. The second topic represents a timely contribution of radiation chemistry to a subject of great current interest. The extended $\pi$-system of the fullerene enables it to accommodate up to six extra electrons, to lose electrons in oxidative processes, and to act as a radical sponge. The effect that functional groups exert on the $\pi$-system is addressed by examining the changes in redox properties, excited states, and associated reaction kinetics. The third topic applies radiation chemical methods in the degradation of environmentally hazardous organic compounds. The study of redox and free-radical reactions of halogenated organic acids considers the fact that these compounds are molecular intermediates in the degradation of aliphatic halocarbons in route to complete mineralization. The radiation chemically induced free-radical mechanisms are also studied in 
order to understand and complement corresponding degradation processes initiated by photocatalysis at semiconductor surfaces.

\section{Quantum Mechanical Studies of Radiation-Produced Intermediates Chipman, D.M.; Carmichael, I.C.;Bentley, J. 219-631-5562}

Highly reactive species produced by radiolysis or photolysis, particularly free radicals and molecular excited states, are being characterized by modern methods of ab initio electronic structure theory to facilitate the interpretation of experimental data on chemical properties of these transient molecules. In recent work, hyperfine splitting in the important HOCO radical, which is formed during radiolysis of acidified aqueous formate solutions and is also a key intermediate in hydrocarbon combustion, has been characterized by high-level calculation. Two isomers have been identified and support provided for the experimental assignment of an observed laser magnetic resonance spectrum to the "trans" form. Structures and fundamental vibrations have been determined for the para-benzodithiyl radical anion, which has only recently been experimentally examined. Comparison to the electronic structures of the related phenylthiyl, phenoxyl, and parabenzosemiquinone anion radicals has led to valuable insights into how the differing spin distributions of these species relate to their very distinctive chemical reactivities. Hyperfine coupling constants have also been calculated for these and related para-substituted phenoxyl radicals. In particular, the question of the planarity of the para-aminophenoxyl radical has been resolved by comparison of the calculated electron spin resonance spectrum to experimental results.

\section{Inorganic Photochemical Processes Ferraudi, G.J. 219-631-7676 \\ $\$ 180,000$}

This project focuses on the photophysics and photochemistry of coordination compounds and the mechanisms of reactions involving complexes in unusual oxidation states. Of particular interest are electronic excited states or energy-rich intermediates with potential energy-storage applications. Techniques in which the chemical systems are perturbed by intense magnetic fields are being exploited to probe these excited states and intermediates to establish the mechanisms of reactions in which they participate. Electronic transitions to the lowest-lying excited states of phthalocyanines are being investigated with time-resolved magnetic circular dichroism (TRMCD). This new technique provides detailed electronic structure information on excited states. In aluminium phthalocyanine chloride, for instance, the TRMCD spectrum indicates that the ${ }^{3} \mathrm{~A}_{1 \mathrm{~g}}{ }^{3} \mathrm{E}_{\mathrm{u}}$ electronic transition reflects a coupling between ${ }^{3} \mathrm{E}_{\mathrm{u}}$ and $\mathrm{n} \pi^{*}$ excited states. The photochemical reactivity of coordination complexes not directly accessible from the ground state is being studied by sequential biphotonic excitations. TRMCD spectra will be used to characterize electronic transitions in the spectra of excited states or reaction intermediates observed in biphotonic irradiations.
24. Microwave Studies of Radiation-Produced Radicals

Fessenden, R.W.; Madden, K.P. 219-631-5354

The molecular electronic, structural, and environmental factors controlling reaction rates and paths for radicals and excited states formed by radiolysis and photolysis are sought by electron spin resonance (ESR), optical, and microwave absorption methods. ESR spectra give definitive electronic and geometrical structures for proof of reaction mechanisms and measurement of acid-base properties. Electron spin polarization (CIDEP) demonstrates the nature of electronic interactions between pairs of radicals or radicals and excited states during formation and reaction. The effect of steric hindrance upon spin trapping rate constants is being measured using the nitrone spin trap dimethyl-1-pyrroline-N-oxide (DMPO) with a number of carboxylated alkyl radicals. The trapping rate constants show that while steric hindrance is less important for DMPO than 2-methyl-2-nitrosopropane, electron withdrawing groups greatly slow the trapping reaction with both traps. Microwave dielectric absorption is used to determine the extent of charge separation in photochemical intermediates to explain changes in reaction mechanism with changes in solvent polarity. Rapid internal charge rearrangements in excited states are identified and their rates measured using the dispersive component of the microwave signal. The yield, injection rate, mobility, decay kinetics, and chemical availability of charge carriers in semiconductor particles and films are also measured by microwave absorption.

\section{Photochemical and Photoelectrochemical Processes for Energy Conversion Kamat, P.V. 219-631-5411 \\ $\$ 210,000$}

This project focuses on the processes that take place at electrode-solution interfaces, and on control of these processes by variation of the electrode structure and composition. Recently nanocrystalline semiconductor thin films with capped and coupled geometries have been deposited from colloidal solutions. When used as electrodes, these films have proven to be photoelectrochemically active with better charge separation characteristics than simple semiconductor films. Fast kinetic spectroscopy and photovoltaic measurements are being carried out to probe photoinduced charge transfer and charge trapping processes in semiconductor colloids composed of metal oxide (e.g., $\mathrm{SnO}_{2}, \mathrm{TiO}_{2}$, and $\mathrm{ZnO}$ ) and metal chalcogenide (e.g., $\mathrm{CdS}$ and CdSe) heterostructures. The kinetics and mechanisms of charge injection processes in dye-modified semiconductor nanocrystallites are also being determined. Of particular interest is the charge transport mechanism in these porous films, which differs significantly from that in bulk semiconductor materials. In situ spectroelectrochemical measurements of nanocrystalline $\mathrm{SnO}_{2}$ and $\mathrm{TiO}_{2}$ films modified with cresyl violet or ruthenium complexes are being carried out to correlate the transient behavior with photocurrent efficiencies and to establish the dependence of heterogeneous electron transfer kinetics on the externally applied potential. Picosecond and nanosecond laser flash photolysis and pulsed electron radiolysis techniques are also being used to probe the excited state and electron transfer reactions of novel sensitizers (squaraines, fullerenes) and aggregates of oxazine and cyanine dyes adsorbed on inert and semiconductor colloids. 


\section{Track Effects in Radiation Chemistry LaVerne, J,A.; Mozumder, A.; Pimblott, S.M.; Schuler, R.H. $\$ 390,000$ 219-631-5563}

Experiment and theory are used to determine the influence of track structure on radiation chemical processes. Ion beams of ${ }^{1} \mathrm{H},{ }^{4} \mathrm{He}$, and ${ }^{12} \mathrm{C}$ with energies up to $30 \mathrm{MeV}$ from the Notre Dame Nuclear Structure Laboratory are used for extensive investigations of hydrocarbon radiolysis. Iodine scavenging techniques are being used to examine radical yields in cycloalkanes, Magnetic field effects on luminescence yields are giving information on the relative yields of single/triplet formation and total singlet formation, is followed from selected product yields and from scintillation yields. The structure of energetic electron tracks and the spatial distribution of the reactive radicals and ions produced in the different phases of water and hydrocarbons are simulated using Monte Carlo methods and experimentally derived cross-sections. The short-time radiation chemistry of the reactants is examined using independent reaction times and random flights calculations, and the effects of track structure on the observed kinetics probed. Stochastic methods are used to simulate the kinetics of radiation-induced ions and excitons in media exhibiting high structural anisotropy in terms of diffusion, nearest-neighbor hopping, and longdistance tunneling, Two-state models including a ballistic contribution are applied to the mopility of electrons and electron scavenging in hydrocarbons,

\section{Influence of Ordered Molecular Assemblies on Photochemical Processes Patterson, $L, K$. 219-631-6163 $\$ 105,000$}

The effects of molecular organization on the enyironment of photoreactive materials and its influence on energy and electron transfer are determined by both spectroscopic and kinetic measurements, These investigations are carried out in lipid molecular assemblies (vesicles, micelles, and especially lipid Langmuir films) to elucidate the mechanisms by which organized media may be utilized to exercise kinetic control over reactions relevant to energy storage, Emphasis is placed on modifying the rates and efficiencies of electron or energy transfer to generate and stabilize reactive intermediates. Monolayer compression studies indicate that the rates of forward electron transfer from excited lipid-functionalized pyrene to dipyridinium cation are sensitive to lipid organization and consistent with two-dimensional diffusion. Flash photolysis measurements in vesicles indicate that electron transfer from the excited pyrene singlet state is more efficient than from the triplet. It is shown that functionalization of methanofullerenes provides a potential path to stable monolayers of fullerenes at the nitrogen-water interface.

\section{Radiation Chemistry Data Center} Ross, A.B.; Helman, W.P.; Hug, G.L.; Carmichael, I.C, $\$ 300,000$ 219-631-6528

Kinetic, spectroscopic, and thermodynamic data for processes in solution involving reactive intermediates, including free radicals and excited states, are being compiled, evaluated, and collected into databases. These compilation efforts are primarily focused on time-resolved radiation chemical and photochemical research. Critical reviews of the kinetic data on decay and reactivity of singlet molecular oxygen in fluid solution and the aqueous solution kinetics of metal ion transients have been completed. A review of the rate constants for reactions of aliphatic carbon-centered radicals in aqueous solution is in progress. Version 2 of the NDRL/NIST Solution Kinetics Database has been released. The database contains rate constants for 10,800 radical processes involving 7,800 different chemical species in fluid solutions. Software is included for finding reactions by chemical names or name fragments or formulas, Searches may also be made by products or by author. The Center provides an on-line service for searching the RCDC bibliographic database containing about 140,000 references and numeric databases containing rate constants for reactions of inorganic radicals in aqueous sqlution and triplet-triplet absorption spectral data in condensed phases. The bibliographic database is used to produce the currentawareness publication. The Biweekly List of Papers on Radiation Chemistry and Photochemistry.

\section{Radiation-Induced Chemical Reactions Schuler, R.H.; Tripathi, G.N.R.; $\quad \$ \$ 900,000$ Patterson, $L . K$; Chateauneuf, $J_{\text {. }} E$. 219-631-7502}

In this project, the radiation chemical reactions of organic molecules are examined in various media. Radiation chemistry is an extremely useful tool for probing electron transfer reactions, which are central to chemical schemes of energy production, storage and utilization. One subject of this study is substituted benzenoid systems, which are capable of a variety of reduction/oxidation reactions and are widely involved in electron transfer processes in nature and in commercial applications. The Raman spectrum of the uncomplexed riboflavin radical has been observed in aqueous solution using electron radiolysis and time-resolved resonance Raman spectroscopy, enabling the interpretation of complexation modes in previously ob:tained spectra in proteins and on metal surfaces. The initial distribution of radicals produced in the radiolysis of hydrocarbons is being probed by gel permeation chromatography in combination with ion scavenging techniques. This approach has made it possible to show that ring opening, previously unobserved, occurs to a significant extent in the radiolysis of cycloparaffins. Reaction of the benzhydrylcation in fluoroform supercritical fluid have been observed with pulsed electron radiolysis and optical absorption detection. Reaction rates with tetramethylethylene are consistent with suitably scaled liquid solution rates, while those with triethylamine indicate preferential cosolvation of the cation. 


\section{Chemical Physics}

\section{Ames Laboratory Iowa State University Ames, IA 50011}

\section{Fundamental Interactions Program}

\section{Chemical Theory}

DePristo, A.E.; Gordon, M.; Ruedenberg, $K$. 515-294-9924

One focus of this research is the theoretical description of the structure and dynamics of metal clusters. The goal is to determine the structure and energy of various clusters and to investigate the relationship between these properties and the reactivity of clusters with different gas-phase molecules. $\mathrm{Ab}$ initio and semiempirical electronic structure techniques are utilized along with classical dynamics. Theoretical developments are directed toward a combination of electronic structure techniques with molecular dynamics. Another effort involves analysis of the kinetics and nonequilibrium structure associated with irreversible or far-from-equilibrium adsorption and catalytic reaction processes on solid surfaces. Another focus is development of rigorous quantitative methods for (i) abinitio electronic structure calculation of PES, in particular based on multiconfiguration self-consistent field approaches; (ii) the identification of chemically relevant global and local characteristics of PES, such as equilibrium structures, reaction barriers, transition states, reaction paths and channels, bifurcations, gradient fields, and interactions between different PES; (iii) extracting, from complex abinitio calculations, explanatory analyses of reactive energy changes in terms of concepts that fit into the nontheorists' intuitive models of molecules in terms of atoms. Applications are made to important combustion-related reactions.

\section{Molecular Beam Photoionization and Photodissociation Studies of Molecules, Clusters, and Radicals \\ $\mathrm{Ng}, \mathrm{C.Y}$. 515-294-4225 \\ $\$ 260,000$}

The goals of this program are (1) to obtain accurate thermochemical data, such as ionization energies and bond dissociation energies, for neutral polyatomic molecules, radicals, and their ions; (2) to study the photoionization and photodissociation dynamics of molecules and radicals induced by the absorption of UV and VUV photons; and (3) to investigate the reaction dynamics and mechanisms of fast radical-molecule and radical-radical reactions. One current focus is on the studies of organosulfur radicals and transition metal carbonyl compounds, and/their fragments. Oxidation of organosulfur compounds, which lare emitted to the atmosphere due to the incomplete combustion of coal and oil, ultimately lead to the formation of $\mathrm{SO}_{2}$ and acid rain. Previous studies indicate that the oxidation rate for organosulfur pollutants increases substantially in the presence of UV radiation. The study of the UV and VUV photochemistry of organosulfur species is relevant to the modeling of atmospheric sulfur chemistry cycles. Motivated to obtain a detailed understanding of the catalytic ability of transition metal ions, experiments have been initiated to examine systematically the energetics and reactivities of transition metal carbonyl compounds and their fragments. Recent focuses have also been expanded to include oxygen-containing hydrocarbon radicals.

\section{Argonne National Laboratory Argonne, IL 60439}

\section{Chemistry Division}

\section{Metal Cluster Chemistry Research Riley, S.J.; Parks, E.K.; Jellinek, J.; Knickelbein, M.B. 708-252-6793

The goal of this program is to understand how cluster structure depends on cluster size and how cluster properties depend on structure, thus contributing to a better understanding of surface chemistry and heterogeneous catalysis. Experimental and theoretical studies of the chemical and physical properties of clusters of catalytically active transition metals are pursued. Experimentally, the reactivity of clusters with small molecules is investigated, including measurements of absolute reaction rate constants and adsorbate binding energies, and determinations of product compositions, the nature of adsorbate binding sites, and cluster geometrical structure. A complimentary experimental effort uses various laser techniques to obtain the optical, infrared, and photoionization spectra of metal clusters and cluster-adsorbate complexes, providing size-specific electronic structure and ionization potential information as well as detailed insights into cluster-adsorbate interactions. The theoretical effort involves studies of stable and metastable structural forms of clusters and interconversion between these, cluster phases and phase changes, stability and fragmentation processes, and pathways of reactive and nonreactive cluster-molecule interactions. The goal is to understand cluster properties and the mechanisms defining them in terms of the nature of interatomic forces.

\section{Photoionization-Photoelectron Research Ruscic, $B$. 708-252-4079 \\ $\$ 453,000$}

The broad goal of this program is to explore and understand the basic processes of interaction of VUV light with atoms and molecules. The main research theme focuses on the generation of highly reactive transient species and their investigation using the photoionization mass spectrometric method and its various derivatives (TPES, PEPICO, ZEKE, MATI). The primary motivation of our research is to render spectroscopic and structural details that are crucial in comprehending the pertinent chemical reactions, to provide reliably accurate thermochemical data, and to generate new insights into the nature of chemical bonds. In general, the emphasis of this program is on species that are either important intermediates in combustion processes or are involved in postcombustion atmospheric chemistry. Occasionally, transient species prominent in other technologically relevant chemical processes, such as chemical vapor deposition for thin-film production, have also been studied. For the purposes of our experiments, these ephemeral intermediates are produced in situ using various suitable techniques, which 
include chemical abstraction reactions using $\mathrm{H}$ or $\mathrm{F}$ atoms, microwave discharge, pyrolysis, sublimation, and on-line chemical synthesis. Our recent measurements on $\mathrm{CH}_{3} \mathrm{O}, \mathrm{CH}_{2} \mathrm{OH}, \mathrm{CH}_{3} \mathrm{~S}, \mathrm{CH}_{2} \mathrm{SH}, \mathrm{CH}_{2} \mathrm{~S}, \mathrm{HCS}, \mathrm{HNCO}, \mathrm{NCO}$, HNCS, NCS, $\mathrm{HOBr}$, and the $\mathrm{C}_{2} \mathrm{H}_{5} \mathrm{O}$ isomers have provided valuable new data that significantly impact the understanding and modeling of combustion of hydrocarbons, alcohols, and sulfur-containing fuels, the RAPRENO $\mathrm{O}_{\mathrm{x}}$ process, and ozone destruction cycles. Our experimental results also play a significant role in testing the most sophisticated ab initio calculations. Currently, effort is being channeled into the completion of a laserbased photoionization apparatus that will add new important capabilities to this program.

\section{Chemical Dynamics in the Gas Phase \\ Wagner, A.; Davis, M.; \\ Harding, L.; Shepard, R.; \\ MacDonald, R.; Michael, J.; \\ Hessler, J.; Gray, S. \\ 708-252-3597}

The project mission is to characterize the gas-phase chemical reactivity of small molecules and radicals, especially those of importance in combustion. The mission is accomplished by combining theoretical studies in the energetics and dynamics of chemical reactions with experimental studies in chemical dynamics and kinetics. The theoretical effort includes both large-scale applications of theoretical methods and the development of new methods that efficiently exploit advanced computer architectures. Of particular interest are the new opportunities provided by massively parallel computers. The experimental effort includes state-resolved low-temperature kinetics studies in flow tubes and shock tube studies of high-temperature kinetics using the newly constructed equipment. This apparatus enables the study of novel reaction systems involving both radical-molecule and radical-radical kinetics. The close coupling between theory and experiment brings a unique combination of expertise to bear on problems of chemical reactivity.

\section{Brookhaven National Laboratory Upton, L.I., NY 11973}

\section{Department of Applied Science}

\section{Combustion Kinetics and Reaction Pathways Klemm, R.B.; Sutherland, J.W. 516-282-4022}

The objective of this research project is to further the understanding of two fundamental, complementary aspects of combustion chemistry, i.e., the kinetics of combustionrelated reactions and the thermodynamics and reaction dynamics of the compounds and transient species involved. This is achieved by (1) determining absolute rate coefficients for gas phase reactions (which involves modeling of reaction mechanisms and theoretical calculations), (2) identifying pathways for multichannel reactions, and (3) studying photoionization and thermochemistry of the compounds and radicals. To accomplish these goals, this experimentally based project utilizes multiple techniques that provide unique capabilities in performing reliable $\mathrm{ki}$ netic measurements over an exceptionally wide range in temperature, $300 \mathrm{~K}$ to $2500 \mathrm{~K}$. In addition, a discharge flow-photoionization mass spectrometer (DF-PIMS) apparatus is employed in determining primary products from multichannel reactions and in performing photoionization studies on reactants, free radicals and stable reaction products. Continually undergoing improvement, the DFPIMS apparatus was designed to be operated on beamline U-11 of the National Synchrotron light Source (NSLS) and thus take advantage of high intensity, tunable VUV light to achieve improved detection sensitivity and selectivity in monitoring free radicals.

\section{Chemistry Department}

\section{Gas:Phase Molecular Dynamics Muckerman, J.T.; Sears, T.J.; Preses, J.; Hall, G.E. 516-282-4368}

$\$ 1,516,000$

Research in this program explores the energetics and dynamics of molecular collision phenomena and the microscopic factors affecting the structure and dynamics of short-lived intermediates in gas-phase chemical reactions. The spectra of small free radicals, the dynamics of photodissociation, and the state-to-state dynamics of gas-phase reactions and energy transfer processes are investigated using both experimental and theoretical methods. The goal of this work is a fundamental understanding of transient species and chemical processes, especially as related to combustion chemistry. Experimental techniques include: laser absorption spectroscopy; pulsed excitation using visible or ultraviolet lasers; laserinduced fluorescence and stimulated emission pumping; time-resolved Fourier transform infrared spectroscopy; and time-resolved fluorescence measurements. These experimental tools are augmented by theoretical studies employing quantal wavepacket propagation to describe the dynamics of molecules in laser fields, as well as time-independent quantal calculations of molecular spectroscopy and dynamics, using discrete variable representations.

\section{Photoinduced Molecular Dynamics}

White, M.; DiMauro, L.;

Beuhler, R.J. 516-282-4345

$\$ 984,000$

The general aim of this program is to elucidate the molecular dynamics of photoinduced processes through state, energy, and time-resolved spectroscopy measurements in an effort to characterize the structure, spectroscopy, and intramolecular dynamics of chemical species relevant to combustion chemistry. Coherent VUV and ultrafast laser sources are used to study ionization, dissociation, intramolecular motion and desorption, the dynamics of which are probed by a variety of state- and energyresolved ionization-based techniques and nonlinear pump-probe spectroscopy. State-resolved dissociation and photoionization measurements focus on the partitioning of energy and angular momentum in elementary photofragmentation processes at low and very high laser intensities. The latter investigate the response of molecules to intense fields well beyond the perturbative regime, which introduces new selectivity and field induced fragmentation pathways. Further studies of the effects of well characterized optical fields on molecular dynamics are under investigation with the ultimate goal of optimal 
control of physical and chemical processes. State-resolved, VUV detection methods are also being implemented in new studies of photoinduced desorption of molecules from surfaces: These studies will examine the role of surface absorbate excitation and energy transfer mechanisms that result in photochemical surface reactions and/or desorption. Other studies utilize in situ, time-resolved, sum frequency generation spectroscopy for probing the structure and dynaimics of molecules on surfaces.

\section{Lawrence Berkeley Laboratory University of California, Berkeley Berkeley, CA 94720}

\section{Chemical Sciencés Division}

\section{Energy Trañsfer and Structural Studies of Moleculès on Sürfacès \\ Harris, 'C.B. \\ 510-642-2814

The goal of this research is to study the dynamics of excited electronic states on surfaces, at interfaces, and in condensed phases and to develop new laser techiniques for studying these dynamics. The research program is both theoretical and experimental in character, and includes nonlinear optical and ultrafast laser techniques in addition to a variety of standard sưrface science tools for characterizing surfaces and adsorbate-surface interactions. Recent work has centered on the development of new techniques bašed on two photon photoemission for studying the dynamics of electrons at interfaces on femtosecond time scales and carrier diffusion in sèmiconductors. The results of this program have a direct bearing on high-speed technological devices and materials, and on other problems of general interest such as the dynamics of electrical transmission in 'conductors on ultrafast time scales and the optical properties of thin films.

\section{Chemical Dynamics}

Lee, Y.T.; Lester, W.A.;

Miller, W:H.; Moore, C.B.;

Neumark, D.; Johnston, H.S.;

Suits, $A$.

510-486-6154

$\$ 1,725,000$

The objectives of this program are to develop the basic knowledge and understanding of the mechanisms and dynamics of elementary chemical reactions that have a major impact on combustion and advanced energy production technologies. Recent emphasis has been to determine the structure and chemical behavior of free radicals, unusual transient species, clusters, and highly-excited polyatomic 'molecules, and to provide microscopic details of primary dissociation and bimolecular processes. These objectives are achieved with a strongly coupled experimental and theoretical-computational approach, using emerging technologies. Dynamical studies use advanced molecular beam and laser techniques, photofragmentation translational spectroscopy, and ion imaging. Kinetics studies employ IR laser flash kinetic spectroscopy and high-resolution UV-VUV laser spectroscopy. New theoretical methods and models are developed both to provide insight into chemical reactivity and the dynamics of reactive processes and also to allow one to carry out forefront calculations to guide and model several of these experimental studies. There are several significant recent advances: lifetime measurements of high-n Rydberg states of NO and Xe reveal the dependence of these lifetimes on collisions and weak electric fields that mix some high-l character with the prepared state. These studies for the first time place the widely used Zero-Electron Kinetic Energy (ZEKE) photoelectron spectroscopy technique on a firm ground. The photodissociation of ozone at $193 \mathrm{~nm}$ revealed a range of excited products, and a substantial yield of highly excited ground electronic state $\mathrm{O}_{2}$ was observed, recently suggested to play an important role in the stratospheric ozone budget. Photochemistry of numerous radical systems have been studied using flash pyrolysis and fast beam techniques; these include methoxy, methyl, acetyl, and allyl radicals; the results yield new information on thermochemistry and dissociation dynamics for these important combustion intermediates. Combined theoretical and experimental studies have been used to probe the properties of the transition state in ketene dissociation, providing a strong test of the basic tenets of unimolecular reaction theories. Theoretical methods continue to be advanced, allowing efficient calculation of the rate of a chemical reaction directly and without approximation. Theoretical and experimental approaches have been combined in an investigation of energy transfer processes in collisions of electronically excited hydrogen molecules. New studies in the coming years will take advantage of the Chemical Dynamics Beamline soon to be commissioned at the Advanced Light Source. This beamline will be a national User Facility promising a new era in the study of primary photochemistry, spectroscopy, and reaction dynamics, making use of the intense ultraviolet light provided by the ALS. The 'Chemical Dynamics Beamline comprises several dedicated molecular beam machines, 'a specially developed high-intensity laser source in the midinfrared, and the highest resolution VUV monchromator in the world; it will be open to qualified investigators from around the country.

\section{Physicàl Chemistry with Emphasis on Thermodynamic Properties} Pitzer, K.S. 510-642-3472

The object of this project is the measurement and the theoretical calculation of the thermodymamic and related properties of novel or prototype systems. Recently, the emphasis has been on ionic fluids under near-critical or supercritical conditions. Binary fluids have been selected and measured that model a pure ionic fluid but have critical points at experimentally accessible temperatures. Critical exponents have been determined by phase equilibria and light scattering methods. Recent results show a clear crossover from Ising to classical (mean field) exponents as the temperature moves away from the critical point. Other recent research has included a comprehensive equation of state for the important system $\mathrm{CaCl}_{2}-\mathrm{H}_{2} \mathrm{O}$ in the range 523 to $900 \mathrm{~K}$. A new theoretical basis was developed for this equation based on mixtures of dipole $\left(\mathrm{H}_{2} \mathrm{O}\right)$ and quadrupole $\left(\mathrm{CaCl}_{2}\right)$ molecules for which Monte Carlo calculations were made. This represents a good first approximation that is refined by small semi-empirical adjustment terms. Investigations often involve collaboration with Lawrence Livermore National Laboratory '(LLNL), Oak Ridge National Laboratory (ORNL), or the United States Geological Survey (USGS). 
Energy and Environment Division

\section{Combustion Chemistry \\ Brown, N.J. 510-486-4241}

Combustion processes are governed by chemical kinetics, energy transfer, transport, fluid mechanics, and the complex interactions among these. The pathways for energy movement and the competition among the pathways determines reaction rates, product yields, and product state energy distributions. Understanding the fundamental chemical processes offers the possibility of optimizing combustion processes. We continue to develop formalisms for Functional Sensitivity Analysis (FSA) to enable the exploration of the relationships between dynamic observables and the potential energy surface structure. Recently FSA has been applied to quantum mechanical studies of reactive scattering of atom-molecule reactions. Results from these studies have provided a wealth of information about how small, localized changes in the potential affect product state distributions, resonance features, and reactivity. Some sensitivity features have been discovered that challenge established concepts of chemical reactivity. New emphasis is being placed on developing and using FSA with quantum rate coefficient calculations to provide a detailed sensitivity map illustrating regions of the potential energy surface that most strongly infiuence calculations of the thermal rate coefficient. A second portion of the effort is concerned with modeling combustion chemistry with particular emphasis on treating problems that require parallel computing for a tractable solution.

\section{Lawrence Livermore National Laboratory University of California Livermore, CA 94550}

\section{Division of Applied Physics}

\section{Chemical Kinetics Modeling Westbrook, C.K. 415-422-4108}

This project emphasizes numerical modeling of chemical kinetics of combustion. Combustion modeling applications in both practical combustion systems and in controlled laboratory experiments are included. Elementary reaction rate parameters are combined into mechanisms, which then describe the overall reaction of the fuels being studied. Detailed sensitivity analyses are used to identify those reaction rates and product species distributions to which the results are most sensitive and therefore warrant the greatest attention from other experimental and theoretical research programs. Experimental data from a variety of environments are combined together to validate the reaction mechanisms, including results from laminar flames, shock tubes, flow systems, detonations, and even internal combustion engines. Particular attention will be given to chemical factors including fuel molecular size and structure, emphasizing differences in combustion of isomers of selected hydrocarbons.
Pacific Northwest Laboratory Richland, WA 99352

\section{Molecular Science Research Center}

\section{Chemical Structure and Dynamics Colson, S.D. 509-375-6882

Chemical reaction dynamics at condensed-phase interfaces are studied using a suite of techniques designed to achieve a molecular-level understanding of these processes, for insight into condensed-phase chemistry and to develop and validate abinitio theories: The approach includes (1) synthesis of unique and well-characterized surfaces and interfaces by controlled deposition of atoms, molecules, and clusters; (2) characterization of surfaces and interfaces by atomic-resolution surface mapping combined. with molecular scattering and diffusion studies; (3) synthesis of atomic and molecular clusters that mimic the structures of surface sites and of solvated species in solutions and at interfaces; (4) laser methods for studying atoms and molecules with time resolution sufficient to measure chemical dynamics in real time; and (5) direct photon and/or electron excitation of surfaces, interfaces, and molecules to model chemical processes important in mixed waste storage (radiolysis) and in the energetic destruction of wastes.

\section{Molecular Theory and Modeling: Dunning, T.H., Jr:; Garrett, B.C. $\quad \$ \mathbf{2 , 4 2 5 , 0 0 0}$ 509-375-6863}

The molecular theory and modeling project is designed to increase understanding of molecular processes important in environmental chemistry. The project integrates ab initio studies of fundamental molecular processes in model systems with modeling of the complex molecular systems found in the environment. Five research areas are emphasized: (1) structure and properties of aqueous clusters and the energetics and dynamics of molecular processes involving such clusters; (2) structure of molecules and the energetics and dynamics of molecular processes in aqueous solutions; (3) structure and energetics of ion-ligand complexes (such as crown ethers) and the dynamics of complex formation in aqueous solutions; (4) binding of molecules to soil minerals and the dynamics of molecular processes at the interface of minerals and aqueous solutions; and (5) energetics and dynamics of molecular processes occurring at the interface between amorphous materials, e.g., glasses and aqueous solutions. This knowledge will further the development of new separations processes for the treatment of wastes, the construction of reliable models of contaminant transport and transformation in soils and groundwater, and the assessment of the stability of long-term waste storage forms. 


\section{Sandia National Laboratories, California Livermore, CA 94551}

\section{Combustion Research Facility}

\section{Turbulent Reacting Flow Research Barlow, R.S.; Schefer, R.W.; Paul, P.H.; Chen, J.H.; Najm, H. $\$ 1,300,000$ 510-294-2688}

This experimental and computational research project is directed toward an increased understanding of the coupling between chemical kinetics and turbulent mixing in reacting flows. Current research efforts address fundamental issues, such as the effects of differential diffusion; unsteady strain and flame curvature; the influence of heat release on the scalar dissipation field in nonpremixed flames; the geometric properties of turbulent premixed flames; and the role of turbulence-chemistry interactions in the formation of pollutants. Quantitative techniques for simultaneous imaging of multiple scalars are used to determine the spatial structure of turbulent reaction zones. The temporal evolution of flame structures is investigated by obtaining two co-planar images of $\mathrm{CH}$ with a variable time delay. The influence of turbulent mixing on thermochemical states is determined by simultaneous point measurements of $\mathrm{NO}, \mathrm{OH}$, the major species, temperature, and mixture fraction. These detailed multiscalar data reveal instantaneous relationships among scalars and constitute a unique basis for evaluation and refinement of turbulent combustion models. Fundamental aspects of reacting flows are also studied computationally by direct numerical simulation (DNS), where all scales of fluid motion are computed. A current focus is the inclusion of realistic chemical kinetic mechanisms in the DNS calculations.

\section{Chemical Dynamics and Kinetics Chandler, D.W.; Miller, J.A.; Rohlfing, E.A.; Hayden, C.C.; Durant, J:L.; Taatjes, C.A. 510-294-3132 \\ $\$ 1,700,000$}

The goal of this research is to understand the details of fundamental chemical processes that occur in combustion. Experiments in chemical kinetics use approaches such as laser-photolysis/laser-induced fluorescence, long-path IR absorption, mass spectrometric and laser diagnosed flow-reactor studies, and high-temperature shock-tube measurements. Recent systems of interest have included the reactive systems $\mathrm{OH}+\mathrm{CH}_{4}$ and $\mathrm{NH}_{2}+\mathrm{NO}$ and the collisional quenching of electronically excited NO. These experimental studies are aided by quantum chemical and statistical theoretical calculations. Experiments in chemical dynamics emphasize collecting data for elementary processes and individual molecules resolved to $a$ quantum-state level. Techniques utilized include ion imaging of unimolecular and bimolecular reactions, femtosecond time-resolved approaches (transient absorption, photoelectron spectroscopy, and stimulated Raman scattering), and linear and nonlinear laser spectroscopies. Recent applications have included ion-imaging studies of the product angular distributions from the $\mathrm{H}+\mathrm{D}_{2}$ reaction and the speed and internal state distribution of the $\mathrm{HI}$ product from the $\mathrm{H}+\mathrm{HI}$ reaction. Femtosecond timeresolved applications include investigations of internal conversion, vibrational energy redistribution, and dissociation. Recent spectroscopic studies have emphasized the application and development of two-color resonant fourwave mixing and laser-induced grating techniques for molecular spectroscopy and photodissociation dynamics

\section{Combustion Research Facility (CRF) Diagnostics Research: Nonlinear Spectroscopic Processes} Farrow, R.L.; Rakestraw, D.J. 510-294-3259

$\$ 548,000$

This project involves the development, support, and application of nonlinear spectroscopic diagnostics for Combustion Research Facility programs. Emphasis is on coherent anti-Stokes Raman spectroscopy (CARS) and resonant four-wave mixing techniques for combustion measurements. CARS is a relatively mature technique that provides spatially and temporally precise measurements of temperature and major species concentrations. Degenerate four-wave mixing (DFWM) has recently emerged as a coherent diagnostic roughly similar to CARS but offering greatly increased sensitivity. Current work is focused on investigations of fundamental issues involved in quantitative applications of DFWM. Topics include experimental studies of isolated DFWM line shapes and intensities as influenced by collisional and Doppler broadening, electronic quenching, predissociation thermal-grating generation, and laser saturation effects. High-resolution pulsed laser systems, in both the UV and infrared wavelength regions, are used for detailed spectral studies. The experimental results are compared to theoretical calculations, with one goal being the development of quantitative models for spectral analysis software. Computer codes for analyzing CARS and DFWM spectra are being developed and made available to diagnostics and combustion researchers. Polyatomic molecules have been detected by exciting infrared transitions, an approach that should dramatically widen the class of species detectable by DFWM.

\section{Flame Chemistry: Modeling and Experiments \\ Miller, J.A.; Kee, R.J.; Rakestraw, D.J. 510-294-2759}

$\$ 920,000$

This research program represents an integrated effort to understand the chemistry of combustion both qualitatively and quantitatively through the development of predictive mathematical models. There are three aspects of the program: (1) the mathematical modeling of flame experiments and other macroscopic experiments where chemistry is a critical factor, (2) the theoretical prediction of rate coefficients and product distributions of critical elementary reactions using a combination of statistical and dynamical methods in conjunction with ab initio potential energy surfaces, and (3) low-pressure flame experiments in which laser-induced florescence and mass spectrometry are the principal diagnostic tools. The focus of the research is on combustion-generated pollutants (nitrogen oxides, soot and its precursors, and other air toxics) and on limit phenomena in combustion (flammability limits, extinction limits, etc.) 
49. Combustion Research Facility (CRF) Diagnostics Research: Novel Techniques and Strategies

Trebino, R,; Paul, P.H. 510-294-2893

The research goals of this project include the conception and development of novel laser-based diagnostic techniques for Combustion Research Facility programs. New techniques involving ultrafast phenomena, wave-mixing, and thermo-acoustic scattering as well as new strategies in planar laser-induced fluorescence (PLIF) and resonant multiphoton excitation are being actively pursued. Frequency-resolved optical gating techniques provide full characterization of single ultrashort laser pulses. These new capabilities and recently developed ultrashort-pulse lasers are being exploited further to develop transient absorption and time-domain resonant wave-mixing combustion diagnostics for measurements of temperature, pressure, and relative concentrations. Investigations of thermo-acoustic scattering processes, exposed in degenerate four-wave-mixing research, are being pursued for potential application as diagnostics of velocity, viscosity, temperature, and concentration. Time-resolved PLIF for quantitative two-dimensional measurements are limited by low signal strengths and a strong sensitivity to quenching processes. Studies of collisional energy transfer and quenching processes are leading to predictive models of quenching cross sections for molecules such as NO and $\mathrm{OH}$. Results from these and other fundamental studies, combined with new laser or camera technologies, are providing new capabilities to combustion researchers.

\section{Atomic Physics}

\section{Argonne National Laboratory Argonne, IL 60439}

\section{Physics Division}

\section{Accelerator Based Atomic Physics Young, L.; Dunford, R.W.; Gemmell, D.S.; Kanter, E.P. 708-252-8878}

This program focuses primarily on atomic structure measurements with an emphasis on precision measurements useful in testing many-body relativistic atomic structure calculations. The experimental work is principally conducted at special facilities located within the Physics Division, ATLAS and BLASE. The ATLAS heavy-ion accelerator provides ion beams of narrow energy spread and continuous energy variability, making it ideal for the study of the atomic physics of highly ionized atoms. In 1994, the ATLAS-based heavy ion program continued the development of a two-foil technique to measure ultrashort lifetimes in the $100 \mathrm{fs}$ to $10 \mathrm{ps}$ regime for highly charged ions. In addition, a program to measure the spectral distribution of the two-photon decay in helium-like $\mathrm{Kr}$ was initiated. This spectral shape provides a sensitive probe of the calculation of the transition probability for this decay and has never previously been measured, although nonrelativistic theoretical predictions exist. The VUV spectroscopy programs at ATLAS shifted emphasis from two-electron systems to three-, four- and five-electron systems where initial measurements were made on multielectron Kr. Collision studies of fast highly-charged ions with $\mathrm{C}_{60}$ were extended to search for the weak photon emission branch of the collisionally-excited system. A follow-up to previous lifetime studies using the fast-beam laser method at BLASE demonstrated that an alternative technique, time-correlated single photon counting, could easily yield comparable accuracies and holds promise for unprecedented accuracy.

\section{Synchrotron Radiation-Based Atomic Physics \\ Young, L.; Gemmell, D.S.; \\ Kanter, E.P. \\ 708-252-8878}

$\$ 790,000$

Our research program in atomic, molecular, and optical (AMO) physics using $X$ rays from synchrotron radiation sources has two major components: (1) ongoing experiments at the National Synchrotron Light Source (NSLS) at Brookhaven National Laboratory, and (2) preparations for AMO physics at the Argonne Advanced Photon Source (APS). The primary goal of the program is to increase the understanding of photon-atom interactions-high-photon energies. Specifically, the intense X-ray probe will enable detailed studies of structure and dynamics of atoms and molecules in a regime where relativistic and quantum electrodynamic effects play a major role. Currently, we jointly operate beamline X24A at NSLS in collaboration with the National Institute of Science and Technology. At NSLS we have (1) initiated studies that will help unravel the complex decay of an inner shell hole by coincidence techniques, (2) developed resonant Raman spectroscopies to reveal previously unresolved spectral features, and (3) studied photoabsorption in a number of simple atomic systems. Concurrently, we have been preparing for the commissioning of the APS. As a member of the Basic Energy Sciences Synchrotron Radiation Center (BESSRC), we have undertaken responsibilities in a number of general areas, such as hutch design, crystal monochromater design, and user policy.

\section{Lawrence Berkeley Laboratory University of California, Berkeley Berkeley, CA 94720}

\section{Chemical Sciences Division}

\section{Parity Violation in Atomic Thallium Commins, E.E. \\ $\$ 120,000$}

The purpose of this project is to refine the successful experiment on parity nonconservation (PNC) in atomic thallium completed in 1984. Major improvements in data rate and consequent reduction of statistical uncertainty can be achieved by means of a new laser amplifier system driven by an excimer pump laser, and by improvements in detector sensitivity, without any other changes in the experimental apparatus or method. These modifications will make possible an increase in data rate by at least a factor of 50. We can then measure PNC in thallium at or better than the 5\% level of precision. Since new and sophisticated calculations of the atomic theory can also approach this precision, the long-desired goal of a very precise test 
of the standard electroweak model in atomic thallium will be achieved.

\section{High-Energy Atomic Physics Gould, H.A.; Belkacem, A. 510-486-7777}

\section{$\$ 470,000$}

The goals of this program are (1) to achieve an understanding of the physics of electron-positron pair production and heavy particle capture from pair production using theory and experiment and (2) to search for a charge-parity violating permanent electric dipole moment (EDM) of the electron as small as $10^{-30} \mathrm{e}-\mathrm{cm}$ (thousands of times smaller than the present limit). Recent results include the discovery of a new atomic collision process, electron capture from pair production. In this process, an electron-positron pair is produced by the transient electromagnetic field of a relativistic ion-atom collision, and the electron from the pair emerges from the collision bound to the projectile ion. Capture from pair production is predicted to be an important beam loss mechanism at the Relativistic Heavy Ion Collider. Present activities include (1) extending the measurement of electron capture from pair production to $10 \mathrm{GeV} /$ nucleon collision energies and the capture of particles heavier than electrons, (2) performing calculations of capture from pair production using parallel computing, and (3) constructing a new experiment to search for an electron EDM using laser trapping and cooling.

\section{Atomic Physics Prior, M.H. 510-486-7838}

Studies of the structure and interactions of atomic systems are conducted to prôvivide the most detailed description of their behavior and to stimulate theoretical understanding of the observed phenomena. The approach to this work emphasizes research topics that are best addressed with unique tools and expertise available at Lawrence Berkeley Laboratory (LBL). Currently the program exploits the ability of two state-of-the-art, electron cyclotron resonance (ECR) ion sources at LBL to produce intense, highly charged beams for the conduct of lowenergy $(v<1.0 \mathrm{au}$ ) ion-atom collision studies. Current emphasis is on multiple electron transfer to bare, one, and two electron ions. This includes measurement of magnetic substrates populated in double electron capture, and the production of low-energy $(<20 \mathrm{eV})$ continuum electrons accompanied by transfer to bound projectile states in collisions with $\mathrm{He}$ and more complex targets. Auger electron spectra, and photon spectra from multiply charged ionatom collisions are used to gain insight into population mechanisms and the structure of highly excited states. The program benefits substantially from collaborative efforts with colleagues from outside LBL.

\section{Lawrence Livermore National Laboratory University of California Livermore, CA 94550}

\section{Physics Department}

\section{Spectroscopy and Collision Studies with Highly Charged Ions Produced by Electron Beam Ion Traps}

Schneider, D.; Beiersdorfer, P.;

Marrs, $R$.

510-422-5940

The program goal is to install high value grid connected PV, and demonstrate that doing so meets the peaking requirements of selected circuits, thereby deferring the costly replacement of old, underground $4 \mathrm{kV}$ circuits or other similar circuits which may be reaching the limits of their loads.PV, which is the direct conversion of solar energy into electricity offers the greatest potential value for protecting the environment in the long term. There are no emmissions, no wastes, solid or liquid. There are no moving parts. This project will significantly enhance the potential cost-effective on-grid applications for PV, in turn stimulating production which will further reduce costs resulting in ever most cost effective applications, leading to a sustainable market.

\section{Oak Ridge National Laboratory Oak Ridge, TN 37831}

\section{Physics Division}

\section{Accelerator Atomic Physics Datz, S.; Krause, H.F.; Vane, C.R. 615-574-4984}

The project objective is to achieve a detailed understanding of the interactions of high-energy, multiply-charged ions with gas and solid targets, and with electrons. The facilities used for this research are the EN Tandem Accelerator and the Holifield Radioactive Ion Beam Facility (HRIBF). A unique feature of the EN Tandem Facility is the Elbek high resolution magnetic spectrograph. This is coupled with a new recoil momentum spectrometer to allow complete energy disposition studies. Using heavy ions of different $\mathrm{Z}$ but the same charge state, the $\mathrm{Z}$ dependence of the energy shift of the Binary Encounter peak energy has been studied. To determine the relative importance of electron-electron vs. electron-nucleus interactions in excitation and ionization, neutral and charged ions in collision with He gas are measured, as are the collision partners in coincidence. Electrons contained in a crystal channel can be quantitatively treated as a dense electron gas target. A swift ion passing through the channel can be excited by collisional excitation, by dielectronic processes, or by resonant coherent excitation in which the periodicity of the crystal lattice provides an oscillator, which can separately excite specific $m$ states of the moving ions. The strong phase coherent electric fields that the projectile experiences inside the crystal can also be used to selectively cause constructive and destructive interferences. At CERN in Geneva, lead beams at energies of $33 \mathrm{TeV}$ are 
used to study electron capture from the negative continuum and lepton pair production cross sections as a function of angle, lepton energy, and target $\mathrm{Z}$. In collaboration with Swedish scientists, experiments have been performed at the Stockholm Heavy Ion Storage Ring to measure dissociative recombination between electrons and molecular ions, e.g., $\mathrm{HeH}^{+}, \mathrm{H}_{3}{ }^{+}$.

\section{Collisions of Low-Energy Multiply Charged Ions \\ Meyer, F.W.; Havener, C.C. 615-574-4705}

$\$ 250,000$

In this activity, multicharged ion interactions with atoms, molecules, and surfaces are studied at the lowest attainable energies. At such energies, the stored electronic potential energy of the multicharged ions becomes an appreciable fraction of the total interaction energy, and inelastic collisions depend strongly on the detailed quasimolecular potentials of the interacting systems. Emphasis is currently on merged-beam measurements of absolute electron-capture and ionization cross sections in the energy range from 0.1 to $1000 \mathrm{eV} / \mathrm{amu}$; to provide benchmark data for the evaluation of theoretical approaches under development for this still poorly characterized energy regime, as well as to investigate low-collision-energy phenomena such as orbiting resonances and other cross section enhancements arising from trajectory effects. Exploratory experimental studies of the neutralization of multicharged ions during grazing interactions with metal, semiconductor, and insulator surfaces are also in progress The current emphasis is on characterizing the energy and angular distributions of ejected electrons, and on measuring the angular and charge state distributions of scattered ions in order to better understand the detailed mechanisms by which the multicharged ions' potential energy is dissipated as the ions are neutralized at the surface.

\section{Theoretical Atomic Physics Strayer, M.; Reinhold, C.O.; Schultz, D.R. 615-574-4590}

$\$ 325,000$

Computational and mathematical techniques are applied to interpret interactions between atoms, ions, electrons, and photons over a wide range of energies, from a few electronvolts to ultrarelativistic energies. Emphasis is on processes involving highly charged ions of interest in fusion plasmas, intense laser pulses, accelerator-based atomic collision experiments, and transport of ions through solids and near surfaces. The techniques used include numerical lattice solutions of the time-dependent Schrodinger and Hartree-Fock equations by basis-spline collocation methods, Monte Carlo evaluation of Feymman diagrams, Born expansion and distorted wave techniques and coupled-channel approaches. Calculations using several large codes on a massively parallel computer are done routinely. Applications have been made to processes at nonrelativistic energies such as capture and ionization, in ion-atom collisions, and multiphoton ionization by intense laser beams. Recent studies include multiphoton and collisional ionization of helium atoms, and neutralization of slow, highly charged ions near a conducting surface. Successful interpretations have been provided for recent experiments on cusp electrons and post-collision effects in ion-atom collisions. The program on relativistic collision physics is focused on phenomena important in accelerator and detector design (e.g., pair production with capture and free pair backgrounds in heavy-ion colliders). Detailed comparisons have been made of perturbative and lattice treatments of pair production with captưre.

\section{EN Tandem Operations \\ Vane, C.R.} 615-574-4497

$\$ 250,000$

The EN Tandem Van de Graaff is operated for atomic physics research. A wide variety of light ions and multiply-charged heavy ions are furnished by the EN Tandem at $\mathrm{MeV}$ energies for the accelerator atomic physics group and for outside users from other divisions of Oak Ridge National Laborätory (ORNL), universities, and industry. Neutral beams of 0.1 to $0.5 \mathrm{MeV} /$ nucleon carbon and oxygen have recently been added. Terminal voltages up to $7 \mathrm{MV}$ are routinely available and ion sources are sufficiently versatile to provide beams of all ions from protons through fluorine and silicon through chlorine, as well as beams of many heavier ions including nickel, iodine, gold, and uranium. A VAX-11/7750 and a Macintosh PC/ CAMAC-bàsed data aćuiisition system, an Elbek màg: netic spectrograph with position sensitive detectörs, a high-resolution electron spectrometer, $\mathrm{Si}(\mathrm{Li})$ detectors, and a curved crystal X-ray spectrometer are aväilable to users. Recent major beam usage has included the channeling of carbon and nitrogen ions through thin crystals to examine resonant coherent excitation of one-electron ions, coincidence measurements of emitted electrons with recoiling target ions and various priojectile charge states, and measurements of Átiger electrons in coincidence with recoiling helium, neon, and argon atoms:

\section{Chemical Energy}

\section{Ames Laboratory Iowa State University Ames, IA 50011}

\section{Processes and Techniques Prograim}

\section{Organometallic Complexes in Homogeneous Catalysís \\ Angelici, R.J.} 515-294-2603

The aim of this research is to provide an understanding of the details of the process in which organosulfur compounds in petroleum feedstocks are desulfurized. This hydodesulfurization (HDS) reaction is practiced on a very large scale commercially worldwide. As increasingly stringent environmental regulations require further reductions of sulfur in petroleum-based fuel, improvements in the catalytic process are required. From studies of model organometallic complexes of thiophene and related organosulfur compounds, it has been established that $\pi$ bonded thiophenes are activated to undergo reactions that lead to cleavage of the carbon-sulfur bonds in the thiophene. Such bonding on HDS catalyst surfaces would be expected to lead to desulfurization of the thiophene. To test this hypothesis, reactor studies of thiophene with deuterium over molybdenum-based catalysts were performed. The deuterium location in the butadiene product 
is consistent with activation of thiophene by $\pi$-bonding to metal sites on the catalyst surface. Future studies include exploring new approaches to the HDS of dibenzothiophenes, a family of compounds in petroleum that are highly resistant to desulfurization

\section{Chemical Kinetics and Reactivity of Transition Metal Complexes Espenson, J.H. 515-294-5730

The general goal of this project is to understand the homogeneous chemistry that underlies catalytic processes including fuel synthesis and selective oxidation of organic compounds. The concern for the environment and efforts to reduce chemical waste have provided a strong impetus for the development and exploration of new oxidation catalysts. An example is $\mathrm{CH}_{3} \mathrm{ReO}_{3}$, an excellent catalyst for the electrophilic activation of $\mathrm{H}_{2} \mathrm{O}_{2}$ in both aqueous and nonaqueous media. Studies of catalytic oxidations of metal thiolates, thiols, bromide ions, olefins, phosphines, and other interesting reagents are being carried out. The kinetics and mechanisms of such reactions are being examined with a particular emphasis on chemical composition, structure, and reactivity of the active form of the catalyst. The second area concerns metal radicals. These 17-electron organometallic species are produced photochemically, and their reactions with a variety of substrates are being studied by laser flash photolytic techniques. The major effort in this area is now directed toward catalytic reactions yielding nonthermodynamic products, an avenue of considerable potential interest in organometallic and organic synthesis.

\section{Fundamental Investigations of Supported Metal Catalysts \\ King, T.S. \\ 515-294-9479 \\ $\$ 325,000$}

Small metal particles, on the order of 1 to $10 \mathrm{~nm}$, supported on high surface area materials such as alumina, are an important class of catalytic materials. They find numerous applications in the petroleum and chemical industries and in environmental protection (pollution control technology). In addition to being important to DOE objectives, the understanding of the details of the fundamental catalytic processes occurring at the surfaces of these small clusters of metal atoms present challenging scientific problems. Most of our effort in this area is focused on elucidating adsorption and surface reaction behavior that is unique to highly dispersed metal particles. The intent of this work is to probe the synergistic effects of adding a second element; the second element can be another metal to form a bimetallic or it can be a promoter, poison, or a support modifier. This program utilizes a unique combination of solid-state nuclear magnetic resonance (NMR) with various traditional catalytic experiments and model reaction studies (e.g., hydrogenolysis, hydrogenation/dehydrogenation, hydroformylation, and Fischer-Tropsch synthesis). The application of NMR to heterogeneous catalysis and materials science is a new and devolving field. Consequently, a broad area of effort in this program is the development of nuclear spin dynamics and solid-state NMR techniques.

\section{New Synthetic Routes to Layered Catalytic Materials: Organometallic Precursors for Chemical Vapor Deposition Miller, G.J. 515-294-6063 \\ $\$ 78,000$}

This research project involves exploration and development of alternative synthetic strategies coupled with characterization of new solid state materials that have potential applications as catalysts or as energy-storage devices. Current efforts include: (1) use of (mesitylene) ${ }_{2} \mathrm{Mo}$ and (mesitylene) ${ }_{2} \mathrm{Nb}$ as precursors to form reduced chalcogenide halide complexes and mixed metal solids; (2) synthesis of $\mathrm{Nb}$ and Mo cluster compounds containing sulfur using higher oxidation state metal complexes in order to produce single source precursors for chemical vapor deposition of $\mathrm{MoS}_{2}$ and $\mathrm{NbS}_{2}$; (3) characterization of novel $\mathrm{Nb}$ chalcogenide halide solids that demonstrate sufficient structural flexibility so as to isolate molecular fragments in channels and layers; and (4) intercalation studies of layered $\mathrm{Nb}$ halides and chalcogenide halides. The approach involves solution (homogeneous) and heterogeneous synthesis, characterization by X-ray diffraction, electron microscopy, photoelectron spectroscopy, and magnetic susceptibilities. The goals of this research are (1) to find sources of activated metal atoms that may overcome the thermodynamic driving forces when traditional synthetic approaches are used; (2) to examine potential catalytic, chemical, and electronic properties of the products; and (3) to tailor solid state products by appropriate choice of precursor material.

\section{Solid State NMR Studies: Catalytic Chemistry and Materials \\ Pruski, $M$. \\ 515-294-2017}

Transient techniques in nuclear magnetic resonance (NMR) of solids are used to probe the physics and chemistry of materials involved in heterogeneous catalysis, fossil fuels, and material science. Examples include (1) use of two dimensional NMR and selective excitation NMR experiments to study in situ the dynamics of hydrogen and small hydrocarbon molecules on supported catalysts at various pressures and temperatures of up to $800 \mathrm{~K} ;(2)$ studies of reactions of hydrocarbons on various supported metal catalysts (e.g., $\mathrm{Ru} / \mathrm{SiO}_{2}, \mathrm{Cu}-\mathrm{Ru} / \mathrm{SiO}_{2}, \mathrm{Pt} /$ $\mathrm{SiO}_{2}, \mathrm{Ag} / \mathrm{SiO}_{2}$ ) using high-resolution, solid-state NMR of ${ }^{13} \mathrm{C}$ (variable temperature MAS and CP/MAS); and $(3)^{1} \mathrm{H}$ and ${ }^{13} \mathrm{C}$ NMR studies of chemical vapor deposited diamond thin films and ion-conducting glasses. Other projects include development of new research capabilities in solid-state NMR (e.g., fast spinning variable temperature technique for in situ NMR, and application of new nuclear spin dynamics to the studies of surfaces).

\section{Spectroscopic and Kinetic Characterization of Metal Oxide Catalysts Schrader, G.L. 515-294-0519 $\$ 153,000$}

This research is providing new fundamental information about catalysis by metal oxides, including the mechanisms of catalytic reactions, the structure and composition of catalysts, and the properties of surfaces. The metal oxides being investigated are used extensively by industry for selective oxidation, particularly for the activation of paraffins for fuels and chemical production. A complement of 
experimental approaches is being used to perform kinetic measurements and comprehensive catalyst characterization. In situ spectroscopic techniques, such as laser Raman and Fourier transform infrared spectroscopies (FTIR) are emphasized since they can be used to examine functioning catalysts at the elevated temperatures and pressures typical of industrial processes. The goal of this research program is to provide fundamental relationships between structure, composition, oxidation state, or surface properties and catalytic activity and selectivity.

\section{High-Temperature Gas-Phase Pyrolysis of Organic Compounds \\ Trahanousky, W.S. \\ 515-294-2886

The goal of this research is to understand in detail fundamental thermal reactions of organic compounds, especially those related to the pyrolysis of coal and coalderived liquids. Primary products of thermal reactions are often highly reactive neutral species such as radicals, carbenes, diradicals, and reactive molecules (i.e., species with no overall electronic charge, but with an exceptionally reactive bond or group of bonds). Much of the work of this project focuses on reactive molecules that are important in thermal reactions and includes development of novel methods to prepare them and study of their spectroscopic and chemical properties. Studies have concentrated on quinodimethanes, a large class of reactive molecules. The work with reactive molecules has resulted in novel and effective ways of producing diradicals, and the reactions of these intermediates are under study. Recently, several new thermal rearrangements of hydrocarbons and related hetero-atom derivatives were discovered that fit a proposed two-step mechanism that involves formation of a transient diradical by an intramolecular thermal hydrogen-atom transfer. The results suggest that this two-step process is very general and may be a major new way to account for rearrangements which occur when organic compounds are heated to high temperatures.

\section{Argonne National Laboratory Argonne, IL 60439}

\section{Chemical Technology Division}

\section{Fluid Catalysis Rathke, J.W.; Chen, M.J.; Klingler, R.J. 708-252-4549}

The research activities of this program encompass: (1) high-pressure NMR studies of homogeneous catalytic chemistry in supercritical fluids, (2) catalytic and stoichiometric organometallic processes associated with the production of advanced materials, and (3) catalytic processes for the selective functionalization of methane and other hydrocarbons. The program uses an array of in situ high-pressure spectroscopic and kinetic techniques to explore new catalytic chemistry and catalytic reaction mechanisms at the high pressures and temperatures that are frequently used in industrial chemical processes. Recent research includes the first in situ high-pressure NMR measurements on Shell-type catalysts for the commercial hydroformylation of olefins. Ongoing activities also include the development of a new technique for investigating ceramic precursor processes that use the rf field gradient within a toroid cavity to achieve NMR microscopy at high pressures. Synthetic and reactivity studies of some highly electrophilic and unusually reactive polyfluorophthalocyanine complexes designed to achieve the controlled functionalization of aliphatic hydrocarbons are also underway.

\section{Chemistry Division}

\section{Premium Coal Sample Program Anderson, K.B. 708-252-1928}

This program provides basic coal research scientists with the best available coal samples. The eight carefully selected samples are now available in glass ampoules in quantities to provide for 15 or more years' requirements. The samples have been kept in as pristine a condition as possible through careful control of the conditions in all stages, from sample collection through processing and packaging. The samples have been characterized through the efforts of more than 60 laboratories, and additional characterization is being carried out. The sample stability is monitored through periodic gas analysis. The number of orders has exceeded 840. A newsletter has been issued quarterly to give new information to all recipients of the samples. The Users Handbook for the Argonne Premium Coal Sample program provides a description of the program; analytical information; a bibliography of over 592 articles with author, subject, journal, and coal indices; and a literature summary. Data and relevant information concerning the Argonne Premium coal Sample program will also be made available via the internet using the World Wide Web.

\section{Characterization and Reactivity of Coals and Coal Macerals}

Winans, R.E.; Anderson, K.B.; $\quad \$ \mathbf{\$ 1 , 2 0 0 , 0 0 0}$

Dyrkacz, G.R.; Botto, R.E.;

Carrado, K.A.; Stock, L.M. 708-252-7479

This program seeks to elucidate the chemical and physical structure of the Argonne Premium Coal Samples and their macerals on a global basis and on the molecular level. The primary objective is to establish statistically accurate, rank-dependent models for the Argonne Premium Coal Samples. Extraction and selective chemical procedures are used in combination with a complementary array of powerful instrumental techniques. The instrumental approaches include laser desorption, high-resolution, and tandem mass spectrometry; NMR spectroscopy and imaging; synchrotron X-ray spectroscopy, imaging, and scattering; and neutron scattering. The emphasis is on large molecules. The synthetic program is designing clays and catalytic materials especially tailored for the conversion of these large molecules. The fundamental information derived from these studies will be used to guide the development of new processes to utilize America's coal, the nation's largest fossil fuel resource. 


\section{Brookhaven National Laboratory Upton, L.I., NY 11973}

\section{Department of Applied Science}

\section{Metal Hydrides Reilly, J.J.; Johnson, J.R. 516-282-4502}

Knowledge of the behavior and properties of hydrogen/ metal systems is essential for the successful implementation of many energy-related processes and applications. The prime concerm of this program is to increase that store of knowledge through the determination of thermodynamic, kinetic, and structural parameters. A particular goal is to relate all pertinent data and hypotheses in order to develop a predictive capability regarding the behavior of a given system. This capability permits the synthesis of compounds having optimum properties for particular applications. Current areas of research are electrochemical characterization of metal-hydrogen systems, preparation of corrosion-resistant and high-capacity metal hydride electrodes, kinetics of the formation and decomposition of hydride phases and the preparation and characterization of a new class of hydrogen bronzes prepared from complex oxides. This involves the use of various tools and techniques including thermodynamic and electrochemical measurements, X-ray diffraction, in situ X-ray absorption spectroscopy (XAS) methods at the National Synchrotron Light Source (NSLS), and electrochemical corrosion measurements.

\section{Chemistry Department}

\section{Catalysis: Reactivity and Structure Andrews, M:A.; Koetzle, T.F.; Bullock, R.M.; Hrbek, J:; $\$ 1,838,000$. Rodriguez, J.A. 516-282-4347}

This program probes selected fundamental aspects of chemical catalysis from a multifaceted perspective. Reactivity-structure correlations are at the heart of the group's efforts related to heterogeneous catalysis. Emphasis is placed on understanding the effects of catalysts promoters (alkali metals) and poisons (sulfur) at a molecular level, and on understanding the distinctive catalytic behaviors of bimetallic surfaces that may serve as models for industrial bimetallic catalysts. Catalytic reactions on metal surfaces are investigated by ultrahigh-vacuum surface science techniques, infrared spectroscopy, and high-pressure kinetics, while the structures of the active surfaces and adsorbates are probed by a variety of methods, including X-ray and ultraviolet photoelectron spectroscopies at the National Synchrotron Light Source (NSLS). In addition to these studies, novel in situ diffraction studies are being undertaken at the NSLS in order to understand the formation and transformations of zeolitic materials under both synthesis and catalytic reaction conditions. Transition metal hydride complexes provide the unifying theme for efforts in homogeneous catalysis. Reactivity studies are designed to elucidate the factors that determine the rates and mechanisms of $\mathrm{M}-\mathrm{H}$ bond cleavage in these compounds. The knowledge gained from these studies is then being utilized to develop catalytic systems capable of effecting new types of hydrogenation reactions. In addition, the group is pioneering novel aspects of metal-carbohydrate chemistry, in which catalytic reactions involving metal hydride complexes may ultimately lead to new approaches to the utilization of renewable biomass organics. Uniquely accurate structural data for both metal-hydride and -carbohydrate compounds, which enhance our understanding of their chemical reactivity, are provided by neutron diffraction studies at the High Flux Beam Reactor (HFBR).

\section{Lawrence Berkeley Laboratory University of California, Berkeley Berkeley, CA 94720.}

\section{Chemical Sciences Division}

\section{High-Energy Oxidizers and Delocalized-Electron Solids Bartlett, $N$. 510-642-7259}

The aim of this work is the synthesis and characterization of new two-and-three dimensional solids that may be useful in electrical energy storage. Fluorides are emphasized because fluorine is highly electronegative, small, and lightweight; thus, high oxidation-state fluorides such as those of cobalt, nickel, copper, or silver have high oxidizing potential and low formula weights. Emphasis is placed on the thermodynamically unstable fluorides, which have sufficient kinetic stability to be easily stored. Such fluorides are not only powerful oxidizers, but the metal center in each is comparable in electronegativity to fluorine. It is probable, therefore, that some of the thermodynamically unstable fluorides will be metallic or even superconducting (like some copper oxide systems). Access to such fluorides is provided by salts of anions that are thermodynamically stable (e.g., $\mathrm{NiF}_{6}{ }^{2-}$ ). Kinetically stable, thermodynamically unstable fluorides, as reagents, can probably substitute for anodic oxidation processes (Simons process) for the preparation of fluorochemicals. Lithium cations in open-channel fluorides could' provide ionic conductors stable to oxidation. New synthetic routes also provide access to ordered mixed rutile materials (e.g., $\mathrm{MnNiF}_{4}$ ). These should be ferrimagnets. Other "rutile" systems such as MNF (isoelectronic with $\mathrm{MO}_{2}$ ) like $\mathrm{CrO}_{2}$, could be metallic.

\section{Catalytic Conversion of $C_{1}$ Compounds Bell, A.T. 510-486-7.095 \\ $\$ 213,000$}

The purpose of this program is to develop an understanding of the fundamental processes involved in the catalytic conversion of $\mathrm{C}_{1}$ compounds such as $\mathrm{CO}, \mathrm{CO}_{2}$, and $\mathrm{CH}_{4}$ to fuels and chemicals. The effects of metal oxides on the Fischer-Tropsch activity of metals such as $R u$ and $R h$ have been investigated. Electron microscopy together with ${ }^{1} \mathrm{H}$ nuclear magnetic resonance (NMR) reveal that metal oxide promoters decorate the surface of the metal. Cationic vacancies at the perimeter of the oxide islands interact with oxygen atoms in either $\mathrm{CO}$ or $\mathrm{H}_{\mathrm{x}} \mathrm{CO}$ facilitating their further reaction to products. Promoter effectiveness correlates with the Lewis acidity of the cations in the metal oxide. In situ IR studies show that the hydrogenation of $\mathrm{CO}_{2}$ to methane proceeds via the dissociation of $\mathrm{CO}_{2}$ to produce $\mathrm{CO}$. The higher rate of methane formation from $\mathrm{CO}_{2}$ than $\mathrm{CO}$ under identical partial pressures 
of $\mathrm{H}_{2}$ and $\mathrm{CO}_{\mathrm{x}}$ is attributable to the lower coverage of the catalyst surface by adsorbed $\mathrm{CO}$ in the former case. Methane is activated on $\mathrm{Ru}$ at low temperatures (623 $\mathrm{K}$ ) to produce $\mathrm{CH}_{\mathrm{x}}$ and $\mathrm{C}_{2} \mathrm{H}_{\mathrm{x}}$ species. These species can be polymerized to produce higher molecular weight hydrocarbons or used to alkylate other organic compounds.

\section{Transition Metal Catalyzed Conversion of $\mathrm{CO}, \mathrm{NO}, \mathrm{H}_{2}$, and Organic Molecules to Fuels and Petrochemicals \\ Bergman, R.G. 510-642-2156 \\ $\$ 275,000$}

The goals of this project is to develop of new chemical reactions in which transition metals interact with organic materials and to understand how these reactions work and their application to the development of new potentially useful chemical transformations. Several years ago a major discovery on this project was that of the first alkane-transition-metal C-H oxidative addition reactions (C-H activation). Subsequent work has been directed at examining the scope and mechanism of the $\mathrm{C}-\mathrm{H}$.activation reaction and working toward utilizing it in the conversion of alkanes to functionalized organic molecules. Recent activities on this project include (1) use of liquefied xenon and krypton as inert solvents for C-H activation; (2) design of experiments aimed at determining whether weak metal-noble gas and metal-alkane complexes intervene as intermediates in these processes; (3) substitution of indenyl for pentamethylcyclopentadienyl ligands to facilitate migratory insertion reactions in the products of $\mathrm{C}-\mathrm{H}$ oxidative addition reactions; (4) exploratory studies on the extension of $\mathrm{C}-\mathrm{H}$ activation methods to $\mathrm{C}-\mathrm{F}$ activation; and (5) improvement in the techniques utilized for flash kinetic studies aimed at directly measuring the rates of reaction of coordinatively unsaturated $\mathrm{C}-\mathrm{H}$ activating intermediates with alkanes.

\section{Chemical Routes to Tailored Oxide Networks Based on Molecular and Polymeric Precursors Tilley, T.D. 510-642-8939}

Advanced solid-state materials with useful properties increasingly involve intricate three-dimensional networks, characterized by complex stoichiometries (e.g., in ceramic superconductors such as $\mathrm{HgBa}_{2} \mathrm{Ca}_{2} \mathrm{Cu}_{3} \mathrm{O}_{8+d}$ ) and/or metastable architectures (e.g., in zeolites). New generations of materials will undoubtedly result from chemically directed, low-temperature synthetic routes. The approach involves use of synthesis, coordination chemistry, and condensation reactions to build novel three-dimensional networks. Primary targets have been oxide-based materials, which are built from tailored, oxygen-rich precursor molecules. This project involves synthesis and characterization of candidate precursor molecules, and then examination of chemical processes by which a metal oxide building block can be transferred to a growing network. Initial directions are based on the finding that metal complexes of the siloxide ligand $\left.\mathrm{OSi}^{\mathrm{t}} \mathrm{Bu}\right)_{3}$ eliminate isobutylene and water cleanly at remarkably low temperatures $\left(100-200{ }^{\circ} \mathrm{C}\right)$ to form $\mathrm{M}_{\mathrm{x}} \mathrm{Si}_{\mathrm{y}} \mathrm{O}_{\mathrm{z}}$ materials. For example, $\mathrm{M}\left[\mathrm{OSi}\left(\mathrm{O}^{\mathrm{t}} \mathrm{Bu}\right)_{3}\right]_{4}(\mathrm{M}=\mathrm{Zr}$, Hf) complexes undergo very clean conversions at about $100{ }^{\circ} \mathrm{C}$ to homogeneous $\mathrm{MO}_{2} \cdot 4 \mathrm{SiO}_{2}$ materials. The chemistry of this network formation allows control over the growth of $\mathrm{ZrO}_{2}$ nanoparticles at higher temperatures. Other precursors being examined include the Al/P oxide cluster $\mathrm{Al}_{4}\left(\mathrm{O}^{\mathrm{i}} \mathrm{Pr}\right)_{8}\left[\mathrm{O}_{2} \mathrm{P}\left(\mathrm{O}^{t} \mathrm{Bu}\right)_{2}\right]_{4}$ and the $\left[\mathrm{ZnO}_{2} \mathrm{Si}\left(\mathrm{O}^{t} \mathrm{Bu}\right)_{2}\right]_{\infty}$ polymer. The low temperatures at which such conversions take place allow for the formation of networks in refluxing hydrocarbons, thereby offering an alternative to the sol-gel approach to thin films, porous ceramics, fibers, etc. (which usually employs alcohol solvents). Initial experiments also indicate that thermolyses of precursor molecules in the crystalline solid state can generate surprisingly ordered microstructures for the resulting oxide materials. Such observations are followed with attempts to add directionality to the network formation, via added templates or "ancillary" ligands in the precursor that might orient the condensation reactions. The ultimate goal of this research is to provide tailored materials with new and specific structural, electronic, optical, and/or catalytic properties.

\section{Potentially Catalytic and Conducting Polyorganometallics \\ Vollhardt, K.P.C. 510-642-0286}

$\$ 260,000$

This project utilizes the principal investigator's expertise in synthetic organic methodology and organometallic reaction mechanisms in an interdisciplinary approach to the designed construction of polymetallic arrays, anchored rigidly on novel $\pi$ ligands that enforce hitherto unprecedented metallic topologies. It has provided access to a range of new soluble organotransition-metal clusters with great potential as catalysts for known and new organic transformations and as building blocks for novel electronic materials. Recent advances include: (1) the discovery of a rapid synthetic entry into "star" oligocyclopentadienylmetals, containing novel ligands in which a cyclic $\pi$ system is completely substituted along its periphery by cyclopentadienyl units; (2) the development of improved regioselective coupling strategies on route to permetallated linear ter- and quatercyclopentadienyls; and (3) the synthesis of the first metal complexes of hexaethynyl- and hexabutadiynylbenzene.

\section{Los Alamos National Laboratory University of California Los Alamos, NM 87545}

\section{Isotope and Nuclear Chemistry Division}

\section{Transition Metal Mediated Reactions of $\mathrm{SO}_{2}, \mathrm{H}_{2}$, and Other Small Molecules}

Kubas, G.J.; Burns, C.J. 505-667-5846

The binding and chemical conversions of environmentally and energy-related small molecules, particularly $\mathrm{SO}_{2}$ and $\mathrm{H}_{2}$, by transition metal complexes is the main thrust. Synthesis, structural characterization, and delineation of the reactivity patterns of a wide variety of such molecules on 16-electron Group 6 and 7 complexes, $\mathrm{M}(\mathrm{CO})_{3}\left(\mathrm{PR}_{3}\right)_{2}$ and $\mathrm{M}(\mathrm{CO})$ (diphosphine) ${ }_{2}$, are being carried out. A new unsaturated cationic complex, $\left[\mathrm{Mn}(\mathrm{CO})\left(\text { diphosphine) }{ }_{2}\right]^{+}\right.$, was synthesized recently. These highly reactive electrophilic centers have shown four different types of binding/ reactivity: classical ligand binding, nonclassical binding of $\mathrm{H}_{2}$, oxidative addition, and free-radical formation. Regarding potential applications, there is a crucial need for 
lightweight materials for hydrogen storage for vehicle fuel, as well as sensors for hydrogen and other gases. The ligand environments of these complexes will be tuned to develop new materials for gas storage, separation, and sensor technologies. In the chemistry of $\mathrm{SO}_{2}$, a new direction is the design of catalytic platinum and palladium systems that incorporate $\mathrm{SO}_{2}$ into organic compounds such as sulfonic esters $\left(\mathrm{RSO}_{3} \mathrm{R}^{\prime}\right)$, analogous to reactions observed in carbonyl chemistry. Novel methods to catalytically oxidize $\mathrm{SO}_{2}$ to $\mathrm{SO}_{3}$ in air, reduce it to sulfur, or disproportionate it to sulfur and $\mathrm{SO}_{3}$ will also be sought. Early transition metal sulfides such as a new anionic titanium-sulfide cluster will be examined for $\mathrm{SO}_{2}$ activation.

\section{National Renewable Energy Laboratory Golden, CO 80401}

\section{Basic Sciences Division}

\section{Basic Research in Synthesis and Catalysis DuBois, D.L.; Curtis, C.J. 303-384-6171 $\$ 396,000$}

The major objectives of research carried out by the Synthesis and Catalysis Group are the development of catalysts for electrochemical reduction and oxidation of $\mathrm{C}_{1}$ chemicals and the synthesis and characterization of organometallic precursors for preparing semiconductor particles and thin films. Electrochemical reduction of $\mathrm{CO}_{2}$ to methanol would provide an attractive route for converting electricity to a liquid fuel with high energy density. The reverse process, oxidation of methanol to $\mathrm{CO}_{2}$ with production of electricity, would contribute to the development of fuel cells based on direct methanol oxidation. Research is in progress to design, synthesize, and characterize efficient catalysts for these important reactions. The preparation of more efficient and lower cost semiconductor materials would have a significant impact on the cost and performance of solar cells and other devices. Current research efforts in this area are focusing on the development of new precursors to thin films and quantized semiconductor particles. This research involves the synthesis and characterization of new organometallic precursors and the study of their decomposition pathways.

\section{Oak Ridge National Laboratory Oak Ridge, TN 37831}

\section{Chemical Technology Division}

\section{Kinetics of Enzyme-Catalyzed Processes Greenbaum, E.; Woodward, J. 615-574-6835 \\ $\$ 530,000$}

The objective of this program is the study of fundamental kinetics and enzyme catalysis of chloroplast reducing power related to fuels and chemicals production from renewable inorganic resources. Fundamental studies that probe the function of the cellulase enzyme components in relation to their structure are also being performed. Kinetic and mechanistic aspects of hydrogen and oxygen photoproduction will be studied using a unique experimental approach. Areas of investigation include: (1) simultaneous light-driven production of hydrogen and oxygen; (2) separation of the two light reactions of photosynthesis; (3) construction of photosynthetic photoelectrochemical cells; (4) precipitation of metallic catalysts on photosynthetic membranes. Sustained photoassimilation of atmospheric $\mathrm{CO}_{2}$ and simultaneous photoevolution of molecular hydrogen and oxygen has been observed in a Photosystem I deficient mutant, B4, of Chlamydomonas reinhardtii that contains only Photosystem II. The data indicate that Photosystem II alone is capable of spanning the potential difference between water oxidation/oxygen evolution and ferredoxin reduction. The rates of both $\mathrm{CO}_{2}$ fixation and hydrogen and oxygen evolution are similar in the mutant to that of the wild-type $C$. reinhardtii 137c containing both photosystems. The wild-type had stable photosynthetic activity, measured as $\mathrm{CO}_{2}$ fixation, under both air and anaerobic conditions, while the mutant was stable only under anaerobic conditions. The results are discussed in terms of the fundamental mechanisms and energetics of photosynthesis and possible implications for the evolution of oxygenic photosynthesis. Further studies on the recent discovery that palladium is a specific inhibitor of cellulase activities have resulted in considerable progress in our understanding of the mechanism of inhibition with respect to cellobiohydrolase I (CBH I). A major finding is that by using palladium to inhibit the catalytic activity CBH I we have shown that the latter is required to disrupt the integrity of cellulose fibers. This could have important practical implications.

\section{Chemical and Analytical Sciences Division}

\section{Organic Chemistry and the Chemistry of Fossil Fuels \\ Buchanan, A.C.; Britt, P.F.; Hagaman, E.W. 615-576-2168

The program objective is to conduct basic research that provides new knowledge on the chemical structure and reactivity of complex organic materials such as coal, lignin, and biomass. Reaction mechanisms that underpin thermal and catalyzed reactions of coal and lignin are being explored through the use of representative organic model compounds. Silica-immobilized compounds are employed to study interfacial reactions in dispersed solids, and the impact of restricted mass transport on reaction pathways involving free radicals and carbocations. Hydropyrolysis reactions are being investigated isothermally and by temperature programmed reduction with on-line detection by mass spectrometry. Pyrolysis pathways for model compounds containing key coal heteroatom functionality such as ethers and sulfides are being investigated in fluidphase and silica-immobilized forms. Model compounds and polymers containing carboxylic acid functionality are being prepared, and their pyrolysis behavior investigated to explore reaction pathways associated with low temperature cross-linking events observed in the pyrolysis of low rank coals. Solid-state NMR methods are being developed to obtain structure/reactivity information in chemically modified coals and lignins. NMR techniques under development include high resolution solid-state ${ }^{19} \mathrm{~F}-\mathrm{NMR}$, and triple resonance ${ }^{13} \mathrm{C}-\mathrm{NMR}$ techniques that exploit the ${ }^{13} \mathrm{C}$ ${ }^{19} \mathrm{~F}$ dipolar interaction to reveal information on local structure in ${ }^{19} \mathrm{~F}$-labeled organic molecules, polymers, and 
coals. Fluorination chemistry under investigation includes conversion of specific oxygen functional groups in low rank coals into fluoride derivatives using diethylaminosulfur trifluoride and sulfur tetrafluoride. This research will contribute to the specific foundations required for the commercial development of novel processes for the conversion of fossil and renewable resources into chemicals or fuels in an environmentally acceptable manner.

\section{Basic Aqueous Chemistry at High Temperatures and Pressures Mesmer, R.E.; Palmer, D.A.; Simonson, J.M.; Ho, P.C. 615-574-4958}

The aim of this program is to establish basic principles governing chemical and thermodynamic behavior of aqueous solutions of broad classes of solutes at high temperatures and pressures through experimental studies. Advancement in experimental methods and the development of new models for exploring and representing behavior over wide extremes of conditions are important parts of this program. A range of diverse but complementary techniques are employed to obtain precise data to and beyond the critical conditions of water and its solutions. Chemical processes under present study are ionization-ion association, complexation, hydrolysis, solubilities, volatilities, adsorption, and oxidation reduction. Thermodynamic quantities of interest are excess properties and ion-interaction parameters, molar volumes, and reaction thermodynamics. Principal methods in use are: flow calorimetry isopiestic methods, electrochemical cells, electrical conductivity, densimetry, liquid-vapor partitioning, and Raman spectroscopy. New results are leading to models for describing variations with temperature, pressure, and composition for both standard state and excess thermodynamic quantities. Computer simulations to relate macroscopic observations to microscopic quantities have been initiated. Results for this program have major impact in basic solution chemistry, basic hydrothermal geochemistry, steam generation technology, utilization of geothermal resources, nuclear waste disposal, and environmental chemistry.

\section{Heterogeneous Catalysis Related to Energy Systems \\ Overbury, S.H.; Huntley, D.R.; Mullins, D.R.; Grimm, F.A. 615-574-5040}

The objective of this program is to understand fundamentals of catalytic reactions of organosulfur molecules occurring at metallic and bi-metallic surfaces and to understand how these molecules affect and are affected by the surface structure and composition. The approach is experimental but is complemented by theoretical modeling used as an interpretive tool. Three dedicated ultrahigh vacuum systems are used to identify and monitor molecular and atomic adsorbates as a function of environmental variables such as coverage and temperature, to monitor reactions and determine their mechanisms and kinetics, and to analyze surface structure. An associated aspect is the development of modern tools such as alkali ion scattering and synchrotron-based techniques for this purpose. Adsorbates chosen for study include thiols, substituted thiophenes, bi-functional molecules such as benzenethiol and mercapto-ethanol, related organic molecules, and partially deuterated derivatives of these molecules. Substrates are metallic and bi-metallic single crystals, ultrathin films grown on metal single crystals, and these surfaces following sulfidation or oxidation. Current research directions include studies of adsorbate geometry by photoelectron diffraction and X-ray standing waves; adsorption and reaction of methanethiol on ultrathin $\mathrm{Ni}$ films on $\mathrm{W}$ reactions of substituted thiophenic compounds on $\mathrm{Ni}$ surfaces; effects of $\mathrm{O}, \mathrm{H}$, and $\mathrm{S}$ on reaction selectivity in thiophene hydro-desulfurization; and structure of clean and metal dosed bulk oxide surfaces.

\section{Photolytic Transformations of Hazardous Organics in Multiphase Media Sigman, M.E.; Dabestani, R.T. $\$ 373,000$} 615-576-2173

The emphasis of this research program is on fundamental investigations into the influence of the local chemical environment on the photochemistry of hazardous organics. Studies of photochemical events at interfaces (solid/liquid and solid/gas) and in aqueous media are of central importance to this research. Results from the studies conducted in this laboratory will enhance the basic understanding of photochemical processes occurring at industrially and environmentally important interfaces and in aqueous solutions. The photochemistry of polycyclic aromatic hydrocarbons (PAHs) has been the focal point of these studies because of connection between these materials and fossil fuel production and consumption and the status of any PAHs as EPA priority pollutants. Product analysis and in situ spectroscopic techniques are the primary methods used in the investigations. Contributions from electron transfer and singlet molecular oxygen mediated PAH oxidation pathways have been elucidated. Highly polar surfaces, such as that of $\mathrm{SiO}_{2}$, have been shown to have dramatic effects on the photochemistry of weakly interacting organics, as typified by unsubstituted PAHs. Likewise, water has been shown to be a medium that exerts significant influence on the rates and product distributions observed for PAH photochemistry. The mechanistic aqueous photochemistry of PAHs is an area that has been virtually ignored in the past due to difficulties associated with the very low aqueous solubilities of these materials. Among other benefits to be derived from this research is a better understanding of those factors that control the environmental fate and residence times of PAHs and related anthropogenic materials.

\section{Pacific Northwest Laboratory Richland, WA 99352}

\section{Chemical Sciences Department}

\section{Free-Radical Chemistry of Coal Franz, J.A.; Alnajjar, M.S.; Autrey, T.; Linehan, J.C.; Camaioni, D.M. 509-375-2967}

This project develops kinetic, thermochemical, and theoretical information describing the potential energy surfaces of reactions of organic and organometallic free radicals, particularly those involving sulfur, nitrogen, and oxygen, during hydropyrolysis reactions of coal and hydrocarbons. Hydrogen transfer and strong bond scission pathways are examined in kinetic and modeling studies, 
and select bond strengths of heteroatom-containing organic hydrocarbons are determined using redox thermochemical cycles. Semiempirical and ab initio calculations are employed to study hydrogen transfer and radical rearrangement reactions. The project is also involved in developing and applying solid-state NMR techniques for characterization of carbon and heteroatom-containing structures in coal and polymers, and for use of NMR methods for measurement of bond distances and structural topography of complex solid materials.

\section{Separations and Analysis}

\section{Ames Laboratory Iowa State University Ames, IA 50011}

\section{Processes and Techniques Program}

\section{Analytical Separations and Chemical Analysis \\ Fritz, J.S. \\ 515-294-5987

The project objective is to devise practical, innovative methods for analytical separations and chemical analysis. Capillary electrophoresis (CE), and ion chromatography are used to separate and determine anions and metal cations in complex samples. A novel CE method enables neutral organic compounds of very similar chemical structure to be separated effectively without resorting to the use of micelles. New resins and techniques are developed for solid-phase extractions and for chromatographic separations. Resins of small particle size are incorporated into membranes to obtain rapid mass transfer. Chelating reagents and chemicals are prepared for isolation of selected metal ions prior to their measurement by atomic or mass spectroscopy. Low-cost resins are being developed for effective cleanup of toxic metal ions in wastes.

\section{Analytical Spectroscopy Houk, R.S. 515-294-9462}

The basic principles and practical aspects of several important methodologies for ultratrace analysis are studied in this project. Plasma sources for atomic spectroscopy and mass spectrometry are emphasized, particularly mechanistic and analytical investigations of the inductively coupled plasma (ICP). New directions in ICP mass spectrometry include basic studies of the sample introduction and ion extraction processes, development of instrumental methods for removing interferences, and the use of ICP-MS in conjunction with chromatographic separations for measurement of elemental speciation. These ICP studies have resulted in state-of-the-art analytical methodologies that are utilized extensively elsewhere in DOE and in the outside analytical community. New studies in ion trapping and ion formation in electrospray mass spectrometry are also being initiated.

\section{Chemical Analysis at Liquid-Solid Interfaces \\ Porter, M.D.}

515-294-6433

$\$ 166,000$

This project examines new avenues for the design, construction, and characterization of monomolecular films at liquid-solid interfaces. Efforts focus on (1) developing atomic-scale descriptions of the lateral packing of spontaneously adsorbed monomolecular films formed from alkanethiols at gold, silver, and copper surfaces; (2) probing solvent-monolayer interactions at such interfaces with in situ infrared reflection and Raman spectroscopies; and (3) examining the fabrication for molecular recognition monomolecular films formed from organosulfur derivatized cyclodextrins. The atomic-scale arrangements are probed by scanning tunneling, atomic force, and lateral microscopy. The molecular level descriptions are derived primarily from infrared reflection and Raman spectroscopies, optical ellipsometry, electrochemistry, and contact angle studies. The aim is to develop relationships that form a basis for broader correlations between the composition and molecular arrangement (spatial orientation and packing density) of organic interfaces with macroscopic physical and chemical properties (e.g., lubrication, catalysis, adhesion, and chemical analysis). The molecular recognition effort examines the incorporation of size-selective channels in long alkyl chain monolayers as well as the synthesis and fabrication of organosulfurderivatized cyclodextrin monolayers.

\section{Lasers in Analytical Chemistry} Yeung, E.S. 515-294-8062

$\$ 324,000$

With the development of new energy technologies, materials problems, environmental pollution, and health effects present challenges to the analytical chemist. Several laser-based analytical techniques are being developed to gain unique insight into elemental, organic, and gaseous pollutants associated with energy utilization, to study chemical changes of ultrasmall samples, and to probe variations in the surface composition of materials. The research emphasize (1) atomic spectroscopy, particularly studies of the fundamental processes in atom sources such as laser-generated plumes; (2) liquid chromatographic and capillary electrophoretic determination of organic, inorganic, and biochemical species using more sensitive or more selective detectors; (3) laser-based detection of large molecules deposited or adsorbed on surfaces of materials, for example by laser desorption; and (4) real-time spectroscopic probes of laser-initiated gas phase reactions relevant to photochemical vapor deposition and etching schemes. 
Argonne National Laboratory Argonne, IL 60439

\section{Chemistry Division}

\section{Separations Science Related to Nuclear and Hydrometallurgical Technology}

Horwitz, E.P.; Barrans, R.E.; $\$ 1,039,000$

Chiarizia, $R$.

708-252-3653

The objectives of this program are (1) to develop new and improved reagents that may be applied to help solve major problems in environmental management and (2) to elucidate the basic chemistry involved in utilizing these new reagents. The major subdivisions of the program are (1) the study of basic interactions between the extractant and diluent with the goal of achieving major alterations in extractant behavior, particularly with regard to both enhancing extraction efficiency and improving the physical properties of the system; (2) the design, synthesis, and characterization of new classes of multifunctional extractants that show extraordinary selectivities of selected metal ions; and (3) the design, synthesis, and characterization of new classes of aqueous soluble nonphosphorus complexing agents, which show unique properties for selected metal ions, All four objectives are directed toward application in nuclear technology, such as actinide separations, waste processing, by-product recovery from nuclear waste, and hydrometallurgical procesșing.

\section{Brookhaven National Laboratory Upton, L.I., NY 11973}

\section{Department of Applied Science}

\section{Structure and Function in Electrochemistry Adzic, $R$. 516-282-4522}

$\$ 257,000$

The objective of this program is to enhance the understanding of the relationship between the structure of an electrode surface and its function in an electrochemical process. A unique feature of this work is the emphasis on in situ determination of the structure of an electrode surface with atomic resolution during the course of an electrochemical reaction, i.e, the identification of atomic geometry of the reaction sites, as well as the identification of adsorbates, intermediates and products, with molecular specificity. Besides insights into fundamental surface electrochemistry and electrocatalysis, the results will haye potential applicability in electrochemical energy conversion, electroorganic synthesis and sensors. X-ray scattering (utilizing the National Synchrotron Light Source) scanning tunnelling microscopy, (nonenhanced) Raman scattering and Fourier transform infrared spectroscopy will be the primary in situ probes. Specific studies will focus on establishing the correlations between the surface structure and its electrocatalytic activity, determination of structure of metal and anion adsorbates and exploring of laws governing the ordering of the adsorbates on surfaces, examining the origins of the reconstruction of electrode surfaces and exploring the new metal-metal oxide catalysts for methanol oxidation.
91. Microparticle Analysis by Laser Spectroscopy

Tang, I.N.; Fung, K.H. 516-282-4517

$\$ 243,000$

Many areas of scientific research and technological development pertaining to DOE's missions demand chemical characterization of aerosol particles so minute in size that conventional methods for bulk analysis are simply not applicable. This program focuses specifically on basic understanding and novel application of laser-based spectroscopic. methods for in situ characterization of such suspended microparticles. Because of the nondestructive and species-specific nature of Raman scattering, a unique single-particle Raman-spectroscopy method is currently being developed for composition analysis of microparticles. Progress has been made in establishing the detection limits for various Raman processes on microparticles. Sensitivity enhancement by resonance Raman scattering is being investigated. In addition, the single-particle levitation technique, in conjunction with spectroscopic tools to probe the physical and chemical state of molecular and ionic species in microparticles, is ideally suited for obtaining insight into the nature of ionic association and solute phase transformation at high concentrations atherwise unattainable in bulk solutions. Thus, discoveries have been made of new solid metastable states that exist only in microparticles, but not in the bulk phase. This research will not only provide the scientific and technological basis for advanced analytical instrumentation, but also lead to a discovery and elucidation of heretofore unknown properties unique to microparticles, thereby extending the frontier of material and catalyst research.

\section{Idaho National Engineering Laboratory Idaho Falls, ID 83415}

\section{Negative Ionization Mass Spectrometry Delmore, J.E.; Appelhans, A.D;:Dahl, D.A. 208-526-2820}

The elucidation of mechanisms for the formation of gas phase ions from high temperature inorganic matrices is the main thrust of this program. There are two main approaches for producing these ion emitters; presynthesis of the desired species before preparing the emitter and production of the desired species via a chemical reaction during emitter operation. Seyeral instruments have been devised to assist in these studies. Tube ion sources have been developed in which large samples of these ion emitting matrices can be pressed into refractory metal tubes and heated to ion emission temperature. These sources can be exchanged between a mass spectrometer and an ion source imaging instrument. Gases can be diffused through the material in the tube so that high temperature gas/solid reactions can be studied in the mass spectrometer. Imaging studies have demonstrated that for the ion emitters studied to date ions originate directly from the surfaces of the inorganic matrices. This work indicates that the emitters are micro chemical factories that either produce the species needed for ion production, or else preserve the presynthesized species, and in both cases provide a matrix conducive to ion emission. This work is leading to methods for custom designing new emitters. 
New theories describing ion emission are currently being formulated to deal with these experimental findings. High temperature redox chemistry to preform the ion of interest is proving to be important in oxide based emitters. Experiments are being formulated to demonstrate similar concepts for the role of reduction for the production of ions of chemically reduced species. The next experimental thrust will utilize a pulsed ion source for determining the extent to which preformed ions are sufficiently stable to be stored on a surface by electrostatic repulsion, followed by electrostatic extraction. A major enhancement of ion current during the extraction cycle will demonstrate the role of preformed ions. A time of flight mass spectrometer has been obtained to assist in these studies. To help develop a more complete understanding of these processes it is necessary to be able to model the motion of ions in electric and magnetic fields. A new modeling code has been developed that enables the ion motion to be predicted in fully asymmetric $3 \mathrm{D}$ electrostatic and magnetic fields.

\section{Lawrence Berkeley Laboratory University of California, Berkeley Berkeley, CA 94720}

\section{Energy and Environment Division}

\section{Repetitively Pulsed Laser/Material Interaction \\ Russo, R.E. \\ 510-486-4258}

$\$ 208,000$

The interaction of a high-powered pulsed laser beam with solid materials offers widespread importance to many areas within the DOE and U.S. industries, including environmental analysis, non-proliferation, materials, electronics, and medical. Importantly, the laser material interaction (LMI) is a powerful technology for chemical separations and analysis. A pulsed, high-powered laser beam blasts constituent elements from any sample material into the vapor phase, which can be analyzed by classical spectroscopic techniques. However, the explosive laser material interaction is not fundamentally defined for general application. This Basic Energy Sciences supported research endeavors to elucidate fundamental mechanisms of the laser-material interaction and to develop laser sampling capabilities for DOE needs in chemical separations and analysis. The research emphasizes the study of repetitive-pulsed laser material interactions at atmospheric pressure. Atomic emission spectroscopic data from an inductively coupled plasma demonstrate changes in the laser material interaction as a function of laser and material properties. Piezoelectric sensors are used to study the propagation of acoustic waves induced in the material by the pulsed irradiation. Probe-beam deflection is employed to determine the onset of material removal and the formation of a laser initiated surface plasma. Fundamental mechanisms describing the laser material interaction are developed by drawing correlations between these acoustic, deflection, and atomic emission data

\section{Oak Ridge National Laboratory Oak Ridge, TN 37831}

\section{Chemical Technology Division}

\section{Chemical and Physical Principles in Multiphase Separations \\ Byers, C.H.; Basaran, O.A.}

615-574-4653

$\$ 539,000$

Electromagnetic fields can enhance drastically rates of transport of momentum, heat, and mass in fluids and thereby offer unparalleled opportunities for improving the efficiency of a wide variety of separation processes. Thus the main goal of this research is to explore by means of fundamental experimental, theoretical, and computational studies the effectiveness of electromagnetic fields in enhancing the efficiency of multiphase separations processes. The primary focus of the program is to develop a fundamental understanding of the effects of electric fields on liquid drops-a topic of great interest in diverse areas of science and technology-with the ultimate goal of using that understanding in devising novel means to dramatically improve transport rates in liquid-liquid systems. Hence, the first and dominant part of the program addresses such issues as (1) equilibria, shapes, and stability of drops, (2) free and forced oscillations of freely floating and supported drops, (3) fluid mechanics of drop formation, (4) breakup and atomization of drops, and (5) interaction and coalescence of multiple drops. A second developing thrust entails an exploratory study of the use of electric fields in enhancing liquid-vapor operations. This part of the program addresses such fundamental issues as (i) effects of external electric fields on bubbles, (ii) formation of bubbles in an ambient flow field, and (iii) free surface flows of multiphase fluids over complicated solid boundaries. Other externally applied force fields, e.g., due to rotation or acoustic fields, are sometimes used to aid the study or to enhance the effectiveness of externally applied electromagnetic fields on drops and bubbles. In addition to multiphase separations, the above-cited two thrusts have ramifications in areas of science and technology that range from cloud physics to ferrohydrodynamics to electrospraying of liquids.

\section{Interactions of Solutes, Solvents, and Surfaces: Adsorption and Supercritical Extraction \\ Cochran, H.D. \\ 615-574-6821 \\ $\$ 356,000$}

The striking properties of solutions in supercritical solvents can be understood in terms of the underlying fluid microstructure and molecular interactions. Fundamental understanding of these properties is the aim of theoretical and experimental studies. Such solutions are important in novel separations technologies such as supercritical extraction and supercritical chromatography and in other technologies as well. Understanding of supercritical solutions of simple fluids has been gained through theoretical studies, molecular simulations, and neutron scattering experiments of solutions of noble gas mixtures. This understanding is being extended to more complex fluids of practical importance to industry-for example, solutions in 'supercritical water and solutions of polymers with supercritical solvents-using molecular simulation 
techniques. Neutron scattering studies of supercritical hydrocarbon systems have been initiated. New molecular simulation codes have been developed for calculations on massively parallel supercomputers. A new equation of state that is simple, as required for many industrial applications, but more accurate than previous simple equations has been extended to polar fluids and is being applied to mixtures. Complementary applied programs are performed to support U.S. industry in areas such as supercritical water oxidation of hazardous wastes, polymerization in supercritical CO!"'” 2 ! and extraction of fatty acids in bioprocessing.

\section{Chemistry of Actinides and Fission Products \\ Toth, L.M.; Hunt, R.D. $615-574-5021$ \\ $\$ 343,000$}

This project is one of only a few remaining fundamental research efforts that are concerned with the physicalchemical characteristics of the actinides and fission products as related to separations schemes. Although the efforts are generally focused on spectroscopic and photochemical approaches, other techniques such as neutron/ $\mathrm{X}$-ray small angle scattering have been employed as a means of identifying more macroscopic properties of these systems (e.g., the sizes and geometries of colloidal species). The fundamental concerns are aimed at defining the chemistry of (1) molten salt systems containing actinides or fission products (which have some potential for separations or waste isolation development); (2) these elements trapped and photolyzed in the controlled environment of a solid matrix (which could encourage novel separations under these conditions); and (3) hydrolytic polymers (namely, the factors controlling their formation, reactivity, and ultimate size, which ultimately influences separations involving these species).

\section{Chemical and Analytical Sciences Division}

\section{Advanced Spectroscopic Methods for Chemical Analysis \\ Hulett, L.D., Jr. 615-574-8955

Spectroscopic methods are being developed for use in fundamental research and applied problems. Optical fluorescence induced in solid materials by positron interactions has been compared to that generated by electron impact. The isolated electron holes produced by annihilation processes relax by mechanisms totally different from the recombinations of electron-hole pairs that result from electron impact. Positron spectroscopy assays of the weathering of paints have continued. The method is extremely sensitive, being able to detect alterations in the outer layers of the paints after only one week of exposure. Paints with pigments have less weather penetration than those without. Scanning tunneling micrographs (STM) suggest that it may be possible to selectively remove the outermost atomic layers, which contain high vacancy concentrations, from silicon surfaces, leaving them atomically smooth. Positron spectroscopy measurements to accompany the STM observations are being considered. This will complement the joint work that is planned with Bell Laboratories in which microelectronic heterostructures will be characterized by positron spectroscopy. Studies of the ionization of organic molecules will be resumed, using new techniques suggested by collaborators at Marquette University. Time permitting, positron stimulated Auger spectroscopy measurements, using loaned equipment from the University of Texas, Arlington, will be started. Other collaborators in this program include Vanderbilt University, Fisk University, The University of Puerto Rico, The Masonite Division of International Paper, and Brookhaven National Laboratory.

\section{Research Development and Demonstration of Advanced Chemical Measurement Techniques \\ Ramsey, J.M.; Barnes, M.D.; Shaw, R.W.; Whitten, W.B.; Young, J.P. 615-574-5662}

$\$ 514,000$

The objective of this research program is to develop new laser-based techniques to improve the sensitivity and specificity of chemical analyses. The methods are directed at analytical problems in the fields of biotechnology, materials science, and environmental monitoring. Areas of research include ultrasensitive fluorescence detection, resonance-ionization mass spectrometry, and nonlinear optical processes. Microdroplet samples are used both to reduce measurement background, enabling the detection of single fluorescent molecules, and to study fundamental cavity quantum electrodynamical effects and their importance on analytical measurements. The application of labeled antibodies to extend single molecule detection to nonfluorescent species is being explored. Real-time diagnostic techniques using gas-phase multiphoton ionization and surface nonlinear optics are being developed for optimizing the growth of thin films by chemical vapor deposition. Another real-time method being studied is the direct analysis of airborne microparticles by laser-ablation mass spectrometry in an ion trap. Some of the planned experiments require laser capabilities in excess of those commercially available. A tunable picosecond laser system with sufficient pulse energy for surface sum-frequency generation and other nonlinear optical processes is being constructed. Difference-frequency mixing will be used to obtain wavelength-tunable IR pulses for vibrational spectroscopy.

\section{Mass Spectrometric $R \& D$ for Inorganic Analyses}

Smith, D.H.; Barshick, C.M.;

Duckworth, D.C.; Riciputi, L.R.

$\$ 330,000$ 615-574-2449

The goal of this FWP is to advance the state of the art in inorganic mass spectrometry. Both fundamental and applied issues are addressed. Areas of emphasis are glow discharge, isotope ratio, secondary ion, and inductively coupled plasma mass spectrometries. Recent applications of the technique include the direct elemental analysis of soil using an rf-powered discharge, and combining an ion trap mass spectrometer with a glow discharge ion source to obtain interference-free mass spectra. Fundamental glow discharge studies are underway to elucidate the formation of rare gas-phase species such as metal argides. A new mass spectrometer that combines an inductively coupled plasma source with a multi-collector sector mass spectrometer should have major impact on isotope ratio measurements, perhaps replacing traditional thermal ionization mass spectrometry in many applications. In 
secondary ionization mass spectrometry, our recent success in in sitù measurement of oxygen isotope ratios has inspired us to address the question of the matrixdependence of mass bias. With staff of the Organic Mass Spectrometry Group we have demonstrated enhanced performance in inductively coupled mass spectrometry when electrochemical preconcentration is used. Improvements in sensitivity by a hundredfold and elimination of matrix effects are the principal benefits.

\section{0. $R \& D$ in Secondary Ion Màss Spectrometry$$
\text { Todd, P.J.; McMahon, J.M.; }
$$$$
\text { Short, R.T. }
$$$$
\text { 615-574-6824 }
$$

This work is about crossing barriers that limit the analytical applicability of secondary ion mass spectrometry (STMS). Our general approach is first to identify the fundamental cause of a barrier, then to characterize it, and finally, to circumvent it by instrumental or chemical development. For example, primary ion damage has been a barrier to obtaining secondary ion images from organic samples. It was recently demonstrated that characteristic secondary ion mass spectra could be obtained from organic samples, even after prolonged exposure to the primary beam. For these experiments, the primary beam consisted of massive cluster ions rather than atomic primary ions. As another example, a method was developed, using time-of-flight mass spectrometry, to accurately measure charging on the surface of insulating samples during analysis by SIMS. It is thus possible to distinguish the contribution of sample charging to the final ion kinetic energy of secondary ions emitted from insulators. Information obtained from these experiments will lead to better charge-compensation schemes for dynamic SIMS imaging of insulators. Insulating samples are among the most difficult of all samples to analyze by SIMS.

\section{Mass Spectrometry $R \& D$ for Organic Analysis \\ VanBerkel, G.J.; Goeringer, D.E.; $\quad \$ \$ 390,000$ McLuckey, S.A.; Ramsey, R.A. 615-574-1992}

The objective 'of this research program is to enhance organic mass spectrometry as an analytical tool via improved understanding of the underlying chemical and physical processes involved. A remarkably broad range of chemical reactions and physical processes can occur within the context of an organic mass spectrometry experiment due to the extremely wide range of reaction conditions that can be established. Unimolecular, bimolecular, and termolecular reactions can occur as well as the wide variety of processes associated with ionization. Ionization by glow discharge, positron annihilation, laser irradiation, and electrospray are of primary interest in this program. Currently research is heavily focused on the fundamentals of electrospray, particularly solution chemistry aspects of the process, and on the combination of electrochemistry with electrospray. The behavior of gaseous ions under a wide variety of conditions continues to be a major focus of this research. Present efforts are concentrated on the behavior of gaseous ions in the quadruple ion trap operated with a relatively high buffer gas pressure. The goal of this work is to determine the extent to which ion internal and kinetic energies can be defined so as to improve the ion trap as a tool for deriving fundamental information from gaseous ions. This information includes structure, reactivity, and energetics. Current efforts include modelling the collisional activation process as well as the kinetics of decomposition. Experimental measurements allow for refinement of the models, which may then become powerful predictive tools. The results of these efforts should enhance both the analytical figures of merit of the ion trap as a mass spectrometer as well as its ability to serve as an ion chemistry research tool.

\section{Chemistry Division}

\section{Chemical and Structural Principles in Solvent Extraction \\ Moyer, B.A.; Sachleben, R.A.; \\ Bonneson, P.V.; Bryan, J.C. 615-574-6718}

$\$ 911,000$

Crown ethers, lariat ethers, and related compounds synthesized and studied in this program are remarkably versatile reagents for achieving selective separations of a variety of species, both cationic and anionic, from aqueous solution. A holistic approach is utilized to investigate the full range of structural and thermodynamic factors underlying the selectivity and efficiency of separations systems of relevance to energy technologies based on solvent extraction and related techniques. The principles of chemical recognition form the basis for probing the influence of complementarity exclusivity, preorganization, strain, inductive effects, and steric factors on host-guest interactions, from relatively simple solvent-solute systems to complex, three-dimensional supramolecular assemblies. Of particular interest is understanding the entire coordination environment of extracted species. Crown ethers and lariat ethers that encapsulate cations or ion pairs by means of appropriately placed substituent groups (alkyl, aryl, hydroxylic, carboxylic, ether, and carbonyl) are being uitilized to effect efficient extraction of metal cations and oxoanions. Separation systems selective for lithium, sodium, cesium, manganese, copper, and technetium are being elucidated. The role of diluents and modifiers has been shown to be critical in the optimization of lipophilicity, solubility, selectivity, and efficiency of extraction systems utilizing macrocyclic extractants. High-resolution NMR, FTIR, X-ray crystallography, and molecular modeling/molecular mechanics provide detailed structural information on extractants and their complexes. Extraction efficiency and selectivity are evaluated by distribution studies, using ion chromatography, ICP-spectrometry, and radiotracer methods. Potentiometry and titration calorimetry provide additional thermodynamic information. Interpretation of the extraction results is aided by use of speciation models derived from the thermodynamic and spectroscopic data by multi-equilibrium modeling using the uniquely powerful program SXLSQI. 
Pacific Northwest Laboratory Richland, WA 99352

\section{Chemical Sciences Department}

\section{Sources in Analytical Spectrometry} Gordon, R.L.; Styris, D.L. 509-376-2540

This research facilitates the direct analysis of solid samples by elucidating the nature of the interactions between trace level analytes and environmental matrices. Speciation of trace elements in and on environmental matrices is determined with X-ray absorption spectroscopy (XAS). Chemical species information is then used, in conjunction with mass spectrometry, to identify chemical and physical processes that will permit efficient, reliable, and direct atomization and/or ionization of analytes for purposes of analytical spectroscopy. Complicated and costly preanalysis chemical processing will therefore be avoided. The research also endeavors to extend XAS to include quantitative speciation in mixtures. Another activity seeks to develop the fundamental physics and chemistry of two innovative ion sources for mass spectrometry. The first approaches solid sampling by directly introducing untreated powder samples into the plasma for analysis by inductively-coupled plasma mass spectrometry. The second seeks to understand and exploit observed major enhancements in the electron capture cross section to excited states of large organic molecules.

\section{Fundamentals of Phase Partittionïng ïn Supercritical Fluids Yonker, C.R.; Fulton, J.L. $\quad \$ \$ \mathbf{3 8 8 , 0 0 0}$ 509-372-4748}

The objective of this program is to describe the molecular interactions underlying separations in supercritical fluids. The scope of these studies spans the range from (1) simple bi-molecular solute/solvent interactions to (2) more complex multi-molecular clustering, chelation, and micellization phenomena as well as (3) ffluid/liquid rinterfacial phenomena. Molecular level studies in supercritical fluids will provide an improved understanding of both fluids and condensed-phase interactions by bridging the gap between the gaseous and liquid states. The approach entails the use of spectroscopic techniques such as FTIR, Raman, NMR, XAFS, and small angle X-ray scattering. The program focuses on the fundamental chemistry that controls the behavior of complex molecular organization in supercritical fluids through experimental and theorefical investigations. These investigations involve probing the underlying chemistry of solute/solvent intermolecular interactions in supercritical fluids and expanding the spectroscopic methods for their characterization. Continued studies seek to characterize angstrom- to micron-sized molecular assemblies in supercritical fluids, e.g., alcohol aggregates, chelates, reverse micelles, and microemulsions. Newily initiated investigations involve the study of the ion solvation and reactions in supercritical water iusing Raman and XAFS spectroscopy. It is anticipated that this program will provide the basis for new and improved analytical separations and for larger scale separations and reactions needed in environmental remediation.
Materials and Chemical Sciences Department

\section{Laser-Based Analytical Techniques Bushaw, B.A.; Whitehead, A. 509-375-2699 \\ $\$ 60,000$}

Optical emission spectroscopy is being investigated as a means of performing direct, fast sample interrogation without chemical pretreatment. Sensitivities for certain analytes in transition metal samples were as low as 23 parts per million per laser pulse. However, even for metal samples with similar vapor pressures and melting points, chemical assays obtained from plume fluorescence differed by $40 \%$ or more from that of the bulk. Self-absorption, electronic densities, and uncertainties in absolute transition strengths have been identified as major contributors to this deviation. These parameters are interrelated with surface morphology, ablation wavelength, and plasma temperature so that clear scaling laws between different metals (much less between metals and insulators) are difficult to infer. Reheating the ablation plume with a second laser increases the plume fluorescence signal after complete dissolution of the ablated material is assured but is again subject to the same uncertainties and cannot yield signal-to-noise ratios ihigher than that obtained immediately after material ablation. Analytical spectroscopic techniques are being developed to probe atomic and electronic densities independent of the ablation process. It is expected that these results will quantify the degree of self-absorption for analyte emission lines as well as provide more sensitive detection of impurities. Optical emission spectroscopy is potentially the simplest, most cost-effective means of rapid analysis of vastly different samples. To this :goal, these studies seek to understand the ibasic physical processes of the photon->sample$>$ photon :energy itransfer.

\section{Heavy Element Chemistry}

\section{Argonne National Laboratory Argonne, II 60439}

\section{Chemistry Division}

\section{Heavy Element Chemistry Research \\ Nash, K.L.; Morss, L.R.; \\ Appelman, E.H.; Beitz, J.V.; Soderholm, $L$. 708-252-3581 \\ $\$ 1,743,000$}

The objectives of this research are to elucidate the fundamental physicochemical properties of the actinides and chemically-related lanthanides in solution, the gaseous state, and in solids. Parallel research efforts address ifelement coordination chemistry and the design of new ligands for f-elements, spectroscopy of the metal ions in various coordination environments, and the influence of f-electrons on the cooperative properties of solids. Thermodynamic and kinetic studies of actinide coordination compounds reveal that cation-size sensitivity/selectivity can be induced with only;partial;preorganization of:ligand donor atoms. Organization of solvent molecules around 'the complexes also is important. :Heavy element photophysics and ;photochemistry studies provide a predictive 
understanding of the consequences of electronic excitation of heavy element compounds with particular emphasis on optically detected nuclear magnetic resonance, photodestruction of complexants, and photoseparation of volatile metal fluorides. Studies of f-electron interactions target development of a predictive understanding of the influence of these ions on bulk properties, such as the interactions between f-ions and transition metals that produce valence instabilities in the solid state. Such materials are candidates for technological applications including catalysts, switches, and sensors. Communicating the importance of basic science for solving problems of critical national significance is a key guiding principle in this research program.

\section{Lawrence Berkeley Laboratory University of California, Berkeley Berkeley, CA 94720}

\section{Chemical Sciences Division}

\section{Actinide Chemistry Edelstein, N.M.; Andersen, R.A.; $\quad \$ 1,465,000$ Raymond, K.N.; Shuh, D.K. 510-486-5624}

Development of new technologies for the use, safe handling, storage, and disposal of actinide materials relies on further understanding of basic actinide chemistry and the availability of trained personnel. This research program is a comprehensive, multifaceted approach to actinide chemistry and to the training of students to address issues in the future. Research efforts include synthetic chemistry to develop new chemical reagents and actinide materials, their chemical and physical elucidation through characterization techniques, and thermodynamic/kinetic studies for evaluation of complex formation. One aspect is the development of complexing agents that specifically sequester actinide ions for the decorporation of actinides in humans and for the separation of actinides in the environment. Extensive studies are underway to prepare organometallic and coordination compounds of the f-block elements showing the differences and similarities among the f-elements and between the $f-$ and d-transition series elements. Optical and magnetic studies on actinides as isolated ions in ionic solids, and in molecules, give information about electronic properties as a function of atomic number. Synchrotron radiation investigations at the Stanford Synchrotron Radiation Laboratory and at the Advanced Light Source provide oxidation state and structural information on actinide material systems of environmental interest.

\section{Nuclear Science Division}

\section{Chemistry of the Heaviest Elements Hoffman, D.C.; Gregorich, K.E. 510-486-4474}

The chemical properties of the heaviest elements are being investigated to explore the architecture of the periodic table of the elements at its furthest reaches and to compare their properties with those of their lighter homologs. Investigations of the dramatic changes in properties which occur in going from the actinides, which end with lawrencium (element 103), to the transactinides are especially important. Methods for studying the chemical properties of elements 102 through 106 even though some of the half-lives are only seconds and only small numbers of atoms can be produced. Chemical separations of elements 102-105 have been used to determine their most stable oxidation states in aqueous solution, thus confirming their positions in the periodic table. Both liquid-liquid extractions and isothermal gas chromatography are used to study and compare the halide complexes of elements 104 and 105 with those of their lighter homologs. The detailed studies of chemical properties of the heaviest elements have shown anomalous trends which cannot be predicted on the basis of simple extrapolations of known periodic table trends. It is important to extend studies to even heavier elements and to compare the results with predictions of fully relativistic calculations. Participation in an international collaboration to use the full range of automated liquid and gas-phase chemical separation systems to perform the first ever chemical studies of seaborgium (Sg, element 106) using the recently reported longer-lived isotopes of $\mathrm{Sg}$ is occurring

\section{Los Alamos National Laboratory University of California Los Alamos, NM 87545}

\section{Isotope and Nuclear Chemistry Division}

\section{Actinide Organometallic Chemistry Burns, C.J. $505-665-1765$}

These studies are designed to advance our understanding of the chemical behavior and electronic structure of complexes of the actinide elements. Synthetic studies of the nonaqueous coordination and organometallic chemistry of the actinides play a key role in extending our knowledge of redox chemistry and metal-ligand bond strengths. Current work is aimed at synthesizing and characterizing complexes containing metal-ligand multiple bonds. These ligands are capable of stabilizing organometallic compounds in higher oxidation states (e.g., hexavalent uranium). Spectroscopic and structural data point to a degree of covalency in metal-ligand bonding in these complexes and provide insight into the behavior of better known analogs (such as uranyl). Synthetic routes for the introduction of oxo and organoimido functional groups at actinide centers have now been extended to yield the first actinide terminal phosphinidene complex, as well as uranium(+6) sulfido and disulfido derivatives. Other studies are demonstrating important differences between the chemistry of tetravalent actinides and that of Group 4 transition metals. Advances in our understanding of the chemical and physical behavior of these elements is critical to the development of advanced chemical processes, analytical tools, and predictive models applicable in actinide recovery, waste treatment, and environmental restoration activities. 
110. Actinide Chemistry in Near-Neutral Solutions

Clark, D.L.

$\$ 139,000$

505-665-4622

The project objective is to provide fundamental physicochemical knowledge pertinent to the behavior of transuranic elements under environmental near-neutral $\mathrm{pH}$ conditions. Advances continued in the application of $\mathrm{C}$ 13 and 0-17 NMR, Raman, and NIR spectroscopy toward determining the stability fields of monomeric and trimeric actinyl(VI) (U,Np) and monomeric neptunyl (V) carbonate complexes. NMR and NIR data provided equilibrium constants and conditions under which a single complex is formed. This information allowed preparation of solution and solid samples for Raman, EXAFS, and XRD analysis. EXAFS and single crystal XRD were used to determine the structures of the two U(VI) carbonate complexes, and to confirm the existence of a planar trimetallic anion, $\left(\mathrm{UO}_{2}\right)_{3}\left(\mathrm{CO}_{3}\right)_{6}{ }^{6-}$. NMR data confirm the formation of the comparable trimeric $\mathrm{Np}(\mathrm{VI})$ species. Large cations (TMA, TBA) afford a relatively high solubility for $\mathrm{Np}(\mathrm{V})$ carbonate complexes; allowed for the study of the solution species by NMR, NIR, and Raman spectroscopy; and revealed the solution conditions under which single species can be prepared. The structures of the $\mathrm{Np}(\mathrm{V})$ mono, bis, and tris carbonato complexes were probed by NMR, Raman, and EXAFS spectroscopy. These data provide a determination of the structures, the number of bound water molecules in the first coordination sphere of the neptunyl ion in carbonate solutions, and show that the carbonate ligands are bound in a bidentate fashion.

\section{Oak Ridge National Laboratory Oak Ridge, TN 37831}

\section{Chemistry Division}

\section{Chemistry of Transuranium Elements and Compounds \\ Haire, R.G.; Gibson, J.K.; \\ Johnson, E.; Peterson, J.R. 615-574-5007}

The objective of the program is to perform systematic investigations regarding the fundamental chemistry, physics, and materials science of actinide metals, alloys, and compounds. The aim is to advance the basic science of these materials and to provide information that will be conducive to technologies relevant to DOE missions. A primary approach is to establish significant trends and/or differences in chemical and physical behaviors. The experimental research focuses on vapor-and solid-state science of the f-elements with the emphasis being on actinides through fermium. The chemistry of the actinides and the transactinides is considered theoretically (quantum and statistical mechanics). Primary scientific disciplines and special capabilities are employed in the experimental studies; these include high-pressure chemistry/physics, high-temperature X-ray diffraction and mass spectrometry, laser ablation mass spectrometry, thermal analyses, optical spectroscopy (Raman, absorption, fluorescence) of solids and novel synthetic techniques. Phase behaviors and high-temperature vaporization behaviors of metals, alloys, and compounds, and the cluster chemistry of oxides are some examples of current areas of investigation.
The primary goals of the research are to define/ understand chemical behaviors in terms of primary scientific concepts such as electronic configurations, thermodynamics, periodicity, etc. Theoretical and computational chemistry are used for interpretation of experimental results, modeling, and deriving scientific information when experimental studies are not feasible. Results from these studies lead to improved understandings of the basic properties of the materials, which are also important in advanced remediation technologies.

\section{Chemical Engineering Sciences}

\section{Lawrence Berkeley Laboratory University of California, Berkeley Berkeley, CA 94720}

\section{Chemical Sciences Division}

\section{Molecular Thermodynamics for Phase Equilibria in Mixtures \\ Prausnitz, J. $M$. 510-642-3592 \\ $\$ 164,000$}

Phase equilibria are required for design of efficient separation processes (e.g., polymer devolatilization and extraction) in the chemical and related industries and for development of new chemical products and materials. In this context, "efficient" refers to optimum use of raw materials and to conservation of energy. Since the variety of technologically important fluid mixtures is extremely large, it is not possible to obtain all equilibria from experiment. Therefore, the objective of this research is the development of molecular thermodynamics for interpretation and correlation of reliable experimental data toward confident prediction of phase equilibria for chemical process and product design. The correlations are expressed through semitheoretical, physicochemical models. Particular attention is given to those materials that may be useful for innovative low-energy-consuming separation processes such as polymers, gels, and polyelectrolyte systems with possible applications in biotechnology. However, attention is also devoted to conventional materials for applications in fuel technology, for recovery of solutes from wastewater, and for proteins used in nutrition or medicine. Development of molecular thermodynamics calls for a combination of theoretical, computational, and experimental work. Further, it requires simultaneous awareness of progress in molecular science and of realistic requirements for engineering application.

\section{Energy and Environment Division}

\section{Turbulent Combustion \\ Talbot, L.; Cheng, R.K. 415-642-6780}

$\$ 339,000$

The objective of this program is to investigate the interaction and coupling between turbulence and combustion. These turbulent combusion processes are characterized by scalar and velocity fluctuations with time and length scales spanning several orders of magnitude. Our approach is to gain a fundamental understanding through 
detailed experimental investigation of idealized laboratory flames with flow and flame geometries amenable to theoretical analysis. These laboratory flames are accessible to detailed interrogation by laser diagnostics. The programmatic emphasis is on obtaining a physical understanding of the coupled effects of turbulence and flame geometry on heat release, turbulent burning rate, stability limits, and flame extinction. Such knowledge is essential for the development of turbulent combustion theories. A primary and continuing effort is the investigation of flames with moderate turbulence under which the chemical reaction rates are high compared to those of turbulence. The burning rate can be inferred from the flame topology (i.e., extent of flame wrinkling caused by turbulence). Our current study is on investigating the relative effects of shear and nonshear turbulence. The research on moderate turbulent flames forms the foundation for the investigation of flames with intense turbulence. Intense turbulent flames are closer simulations of the combustion processes in practical systems where turbulence may alter the reaction rates, cause flame quenching, and affect the formation of pollutants. These flames are more difficult to characterize than the moderate turbulent flame. The use of planar laser-induced fiuorescence techniques to study intense turbulent flames will be a significant effort.

\section{Los Alamos National Laboratory University of California Los Alamos, NM 87545}

\section{Theoretical Division}

\section{Thermophysical Properties of Mixtures Erpenbeck, J.J. 505-667-7195 $\$ 50,000$}

The thermophysical properties of mixtures of particles interacting through simple interaction potentials are evaluated, using both equilibrium Monte Carlo and molecular dynamics, as well as nonequilibrium molecular dynamics. The properties under investigation include the equation of state and transport properties, including mutual and self diffusion, shear and bulk viscosity, thermal conductivity, and thermal diffusion. Fundamental questions arising in the theory of fluids and fluid mixtures are addressed where numerical "experiments" are appropriate. Current research is concentrated on the phase diagram of hardsphere mixtures having diameter ratios no greater than 0.2 and compositions in which the volume of the two species are similar. Theoretical predictions of fluid phase demixing have been made for such mixtures in recent years, contradicting the conventional understanding arising from the Lebowitz solution to the Percus-Yevick integral equation. The Gibbs ensemble Monte Carlo method permits the study of the coexistence of disordered phases, but current implementations are inadequate to overcome the ergodic difficulties attendant upon the disparate hard-sphere interactions. Advanced methods are under development to permit the study of this mixture as well as others having large disparities in the interactions. Recent work has resolved the role of the Einstein-KuboHelfand (EKH) relations in the molecular dynamics calculation of the transport coefficients. The use of periodic boundary conditions is important in the minimization of finite system effects in these calculations, but leads to ambiguities in the EKH formulas themselves. It is found that the EKKH relations present difficulties well beyond those arising in the classical Green-Kubo approach, while the related but unproven McQuarrie relations are almost certainly invalid.

\section{National Institute for Petroleum and Energy Research Bartlesville, OK 74005}

\section{Department of Fuels Research}

\section{Thermodynamic Properties for Polycyclic Systems by Noncalorimetric Methods} Steele, W.V. 918-337-4210

The main objective of this research project is the development of the thermodynamic properties for polycyclic molecules containing five-membered ring(s) via assigned spectra and statistical mechanics. A key goal is attainment of accord between spectroscopic and calorimetrically derived properties for temperatures approaching the critical. A consistent theoretical interpretation is sought for the vibrational modes for ring systems with as many as four rings. This will provide a more fundamental understanding of the foundation of estimation techniques such as group-additivity and molecular-mechanics. Highlights of the first year's research include: (1) For the first time, the fundamental vapor-phase vibrational frequencies were defined completely for furan, pyrrole, and thiophene. Ideal-gas thermodynamic functions were calculated. Anharmonic effects were shown to be negligible [Spectrochemica Acta 1994, 59A, 765-795]. (2) Gas-phase vibrational spectra were obtained for 2-methylfuran, 2and 3-methylthiophene. Agreement between calorimetrically derived entropies and values derived statistically was obtained for a narrow temperature range [Spectrochemica Acta 1994, in press]. (3) Complete gas-phase frequency assignments were made for 2,3-dihydrofuran and 2,5-dihydrofuran [Spectrochemica Acta 1994, in press]. (4) A paper, "Reconciliation of calorimetrically and spectroscopically derived thermodynamic properties at pressures greater than $0.1 \mathrm{MPa}$ for benzene and methylbenzene; the importance of the third virial coefficient," was published [Ind. Eng. Chem. Res. 1994, 33, 157-167]. (5) Papers describing the accord between spectroscopic and calorimetrically derived properties of indole, benzothiophene, benzofuran, benzoxazole, and benzothiazole will be submitted in early summer for peer review and subsequent publication.

\section{Sandia National Laboratories, California Livermore, CA 94551}

\section{Combustion Research Facility}

\section{Analysis of Turbulent Reacting Flows Ashurst, W.; Kerstein, A.R. 510-294-2274 \\ $\$ 414,000$}

The goal of this project is to develop numerical simulation techniques for the understanding of reacting turbulent 
flows. The objective is to show the mechanisms of turbulent mixing and reaction. New simulations of premixed flame motion within an engine-like configuration show how volume expansion interacts with flame distortion to provide exponential growth of flame area. This growth is cancelled by volume confinement during the final part of the combustion period. The structure of a curved nonpremixed methane-air flame has been computed using two-and three-step reduced kinetic mechanisms, and the curvature effects upon flame temperature and the production of oxides of nitrogen have been estimated. The linear-eddy mixing model, unique in its representation of the distinct influences of convective stirring and molecular diffusion, is being used to investigate the spatial correlations of chemical species in turbulent reacting flows.

\section{Advanced Batteries}

\section{Ames Laboratory \\ Iowa State University Ames, IA 50011}

\section{Department of Materials Science and Engineering}

\section{Design and Processing of High Purity and Ultrafine Electrodes for High Performance Nickel/Metal Hydride Batteries Ellis, T.W. 515-294-1366

All major battery manufacturers are aware that their rechargeable $\mathrm{Ni} / \mathrm{Cd}$ products are currently poised on the edge of an outright ban or a heavy tax due to the extreme toxicity of $\mathrm{Cd}$. As a consequence, an acceptable substitute must be found. The $\mathrm{Ni} /$ metal hydride $(\mathrm{Ni} / \mathrm{MH})$ cell has emerged as one of the most promising environmentally acceptable substitutions for current $\mathrm{Ni} / \mathrm{Cd}$ rechargeable batteries in nonautomotive applications for the consumer and industrial market. A key to realization of this potential lies in coupling of the development of both high performance positive and negative electrode materials with the development of efficient, well-controlled electrode processing approaches for each new electrode material. Another key is the close collaboration of a high level research team with a capable and aggressive manufacturer of $\mathrm{Ni} / \mathrm{MH}$ batteries. This coupled development and industrial collaboration can produce not only improved battery performance and effective manufacturability but also will promote a rapid transfer of materials and process research results from the laboratory to the manufacturing floor and marketplace. This program's focus includes the fundamental, systematic development of a significantly improved alloy composition (especially purity level) and electrode fabrication technique for the negative electrode centered on the $\mathrm{AB}_{5}$ (based on $\mathrm{LaNi}_{5}$ ) compound. The objective of this work will be to greatly reduce self-discharge and to double cycling life characteristics of this electrode material over the competing $\mathrm{AB}_{2}$ compounds. Also, focus will be on development of a unique processing approach for the NiOOH positive electrode that will permit fabrication of new ultrafine pitch electrodes. Such an ultrafine pitch electrode with homogeneously dispersed particulate has the potential to increase the energy storage density by about $50 \%$, thereby reducing size and weight of this new generation of $\mathrm{Ni} / \mathrm{MH}$ batteries. This program will be done with intimate contact between the Ames Laboratory and Harding Energy Systems Inc., an emerging domestic battery manufacturer.

\section{Argonne National Laboratory Argonne, IL 60439.}

\section{Chemical Technology Division}

\section{Ion Transport Properties Determined by In:Situ NMR Spectroscopic Imaging} Klingler, R.J. 708-252-9960

This program uses in situ magnetic résonance imaging (MRI) to better define electrode-electrolyte interfaces and solid-state ion transport mechanisms. Work is conducted using a modification of the toroid cayity probe that was designed at Argonne National Laboratory (ANL). This new spectroscopic technique is used for the in situ analysis of the chemical composition at the electrode-electrolyte interface, ion concentration gradients within solid-state batteries, conformational dynamics of polymeric electrolytes, and ion penetration depths within graphite insertion electrodes. Special emphasis is placed on lithium-polymer electrolyte batteries. The lithium foil anodes, as well as the lithium-polymer electrolyte interface, are investigated by a combination of ${ }^{7} \mathrm{Li},{ }^{1} \mathrm{H},{ }^{13} \mathrm{C}$, and ${ }^{17} \mathrm{O}$ NMR spectroscopy. The resulting NMR chemical shift information is used to probe the chemical composition and factors that influence the growth of films at the electrodeelectrolyte interface. In addition, the NMR spectral properties of the polymer electrolyte are probed as a function of distance from the electrode across the lithium ion concentration gradient established in working electrochemical cells to search for specific ion-polymer interactions during the ion transport process.

\section{Chemistry Division}

\section{Template Mediated Synthesis of New Carbon Negative Electrode Winans, R.E.; Carrado, K.A. 708-252-7479}

This study addresses an important limitation of lithium secondary batteries: the performance of the negative electrode. The work is focused on the preparation of the unique carbons that could lead to better lithium secondary batteries and a better understanding of the chemistry and physics of the negative electrode. A chemical approach that is based on the concept of synthesis by design is being pursued to prepare carbon electrode with properties appropriate for an electrode in lithium rechargeable batteries. A unique templating method that is derived from our work with pillared clays has produced the desired porous carbon structures. The physical and electrode properties have been investigated by several methods with promising results. 


\section{Brookhaven National Laboratory Upton, L.I., NY 11973}

\section{Department of Applied Science}

\section{Synthesis and Characterization of Metal Hydride Electrodes \\ McBreen, J.; Reilly, J.J. 516-282-4513

The purpose of this work is to elucidate the structural, thermodynamic, and metallurgical parameters that affect the stability, kinetics, and energy density of metal hydride electrodes. It is focused on alloy development and the application of in situ methods such as X-ray absorption (XAS), X-ray diffraction (XRD), X-ray tomography, magnetic susceptibility, and scanning tunneling microscopy (STM) to determine the roles that various hydride phases and alloying elements play. in hydrogen storage and corrosion inhibition. In situ methods are complemented by ex situ studies such as neutron diffraction, electrochemical, thermodynamic, and metallurgical characterization of candidate alloys. The information thus gained will be used to develop metal hydride electrodes with improved cycle life and energy density.

\section{Lawrence Berkeley Laboratory University of California, Berkeley Berkeley, CA 94720}

\section{Chemical Sciences Division}

\section{Characterization of the Li-Electrolyte Interface \\ Ross, P.N., Jr. \\ 510-486-6226

A detailed understanding of the reactions that occur between metallic $\mathrm{Li}$ and the individual molecular constituents of electrolytes used in $\mathrm{Li}$ batteries will be developed. Ultrahigh vacuum (UHV) deposition methods are used to prepare ultraclean $\mathrm{Li}$ surfaces of preferred orientation. Molecular films of solvent and/or solute molecules are deposited onto the clean surfaces in UHV at a very low temperature. The reaction between $\mathrm{Li}$ and the molecular films is followed using a combination of UHV surface analytical techniques, including Auger electron spectroscopy (AES), secondary ionization mass spectroscopy (SIMS), vacuum UV and X-ray photoelectron spectroscopy (UPES and XPS), and the recently developed variant of XPS termed photoelectron diffraction. The connection between films formed on $\mathrm{Li}$ in UHV and films formed at ambient temperature and pressure on $\mathrm{Li}$ in liquid electrolyte is made by the use of a common spectroscopy, ellipsometry. Using the fingerprint method, the ellipsometric signatures obtained in UHV for different surface layers having various known structures and compositions are used to identify the structure and composition of the film formed on the Li electrode in liquid electrolyte.
Energy and Environment Division

\section{Application of Pulsed Laser Deposition to the Study of Rechargeable Battery Materials \\ Cairns, E.J.; Striebel, K.A. 510-486-5028 \\ $\$ 127,000$}

The aim of this project is to study the performancelimiting phenomena of complex metal oxides and to suggest practical means for improving their performance and lifetime. This is done by preparing thin dense films from these oxides on electronically conductive substrates with the pulsed laser deposition technique. Following film characterization, electrochemical properties of the thinfilm electrodes, such as electrocatalyst kinetics, film corrosion behavior, and/or active species diffusivity, can be measured with standard techniques for geometries with well-defined electrode-electrolyte interfaces. Smooth dense films of the bifunctional oxygen electrocatalysts $\mathrm{La}_{0.6} \mathrm{Ca}_{0.4} \mathrm{CoO}_{3}, \mathrm{La}_{0.6} \mathrm{Ca}_{0.4} \mathrm{MnO}_{3}$, and the lithium intercalation oxides $\mathrm{LiMn}_{2} \mathrm{O}_{4}$ and $\mathrm{LiCoO}_{2}$ have been prepared on stainless steel substrates. Films are characterized with SEM, EDS, XRD, and XPS. The perovskite films are studied with the rotating ring-disk electrode technique for mechanistic studies of the reduction and evolution of oxygen in alkaline electrolytes. Mechanistic dependence on perovskite stoichiometry will be investigated. The $\mathrm{LiMn}_{2} \mathrm{O}_{4}$ and $\mathrm{LiCoO}_{2}$ films are cycled with lithium counter and reference electrodes in liquid organic lithium electrolyte at potentials close to $4 \mathrm{~V}$. Film morphology, lithium diffusion coefficients, and cycling stability will be examined in detail for stoichiometric and later doped oxides.

\section{Fundamental Characterization of Carbon-Based Materials for Electrochemical Systems Kinoshita, $K$. 510-486-7389}

Carbon-based materials have many desirable physicochemical properties for electrochemical systems such as batteries, fuel cells, capacitors, etc. They have excellent corrosion resistance in many electrolytes, acceptable electronic conductivity, good thermal conductivity, and they are available in a host of physical structures. Despite these attractive features and widespread usage, a good understanding of the relationship between the physicochemical and electrochemical properties of carbon-based materials does not exist. A research program on the synthesis, characterization, and modification of carbon-based materials to improve their properties for use in electrochemical systems is being conducted. Electrochemical studies of oxygen reduction and measurements of double layer capacitance were conducted on graphite and carbons synthesized in-house. It was observed that the rate of oxygen reduction increases dramatically when surface defects such as monolayer pits are present on the basal plane. The double layer capacitance of silica aerogel-carbon composites prepared at LBL was determined to be about $80 \mathrm{~F} /$ $\mathrm{g}$ from cyclic voltammetry. Future plans include continuation of the electrochemical experiments with carbons produced in-house, and studies of the electrochemical properties of fullerenes and other carbon nanostructures, which are synthesized at the University of Arizona. 


\section{Los Alamos National Laboratory University of California Los Alamos, NM 87545}

\section{Center for Materials Science}

\section{Development of Materials for Advanced Ni/Metal Hydride Cells \\ Schwarz, R.B. 505-667-8454

This research investigates materials for rechargeable nickel-metal hydride (Ni-MHx) cells with emphasis on (1) a high-energy storage density; (2) high cyclic life; (3) low $\mathrm{H}_{2}$ overpressure operation; (4) low cost; and (5) minimal impact of manufacturing, disposal, or recycling on the ecology. This investigation concentrates on alloys of the type $\mathrm{AB}_{6}$ (e.g., $\mathrm{LaNi}_{5}$ ) and $\mathrm{AB}_{2}$ (e.g., $\mathrm{TiV}_{2}$ ). Alloy powders are prepared by mechanical alloying, a high-energy ball milling technique. This technique enables the preparation of homogeneous alloy powders and two-phase powders with layered morphologies. The program investigates the relation of composition and microstructure to the kinetics of hydrogen absorption-desorption, the hydrogen storage capacities, and the degradation of the hydride material after a number of hydrogen absorption-desorption cycles.

\section{National Renewable Energy Laboratory Golden, CO 80401}

\section{Basic Sciences Division}

\section{Microstructure and Ion Transport in Vanadium Oxide Electrodes for Rechargeable Batteries \\ Turner, J.A.; Zhang, J.G. 303-384-6667}

Vanadium oxide is one of the most promising electrode materials used in secondary lithium batteries and its development is actively pursued by U.S. industry. This task will investigate the fundamentals of ion transport, charging/discharging and the failure mechanisms in these vanadium oxide electrodes, and gain a better understanding of the factors which affect the performance, lifetime, and safety of related rechargeable batteries. Both conventional thermal decomposition and novel sol-gel techniques will be employed to prepare the vanadium oxide electrodes. The correlation between the fractal structure/ morphology of the electrodes and their performance will be studied. These results will allow the prescription of improved preparation conditions leading to optimal thin film morphologies. In collaboration with an industry partner, we will investigate the limiting mechanisms of the charging/discharging process in $\mathrm{V}_{6} \mathrm{O}_{13}$. The deterioration mechanisms of these electrodes in the cycling process will also be examined. Based on both the kinetic data and a new theoretical model/calculation algorithm developed recently in the laboratory, the ion transport processes will be simulated in a solid state battery. For a given thin film combination chosen by the industry partner, the optimum thicknesses of the component layers will be predicted, both for maximizing performance and minimizing material requirements under typical charge/discharge conditions.
The potential failure mechanisms and inherent safety problems in the batteries involving vanadium oxide electrodes will be investigated. Application of expertise in the charging/discharging processes of electrochromic materials is expected to provide cross-fertilization and new insights into the ion insertion kinetics of vanadium oxide and other components of lithium rechargeable batteries.

\section{Oak Ridge National Laboratory Oak Ridge, TN 37831}

\section{Solid State Division}

\section{Rechargeable Thin-Film Lithium Batteries \\ Bates, J.B. $615-574-6280$}

$\$ 200,000$

The purpose of this program is to conduct the research needed for further advancement and commercialization of thin film rechargeable lithium batteries developed at the Oak Ridge National Laboratory. These batteries, based on $\mathrm{V}_{2} \mathrm{O}_{5}$, $\mathrm{LiMn}_{2} \mathrm{O}_{4}, \mathrm{LiCoO}_{2}$, and $\mathrm{LiNiO}_{2}$ cathodes, have potentially many applications as small power supplies that can be fabricated directly onto chips or circuit boards. Several important issues addressed in this research are crucial to the commercialization of this technology. These include long-term cycle testing, determining cell performance as a function of temperature and discharge current density, and devising a reliable method for fabricating stacked, bipolar multicell batteries. Methods for utilizing the lithium phosphorus oxynitride electrolyte discovered at ORNL for improving the performance of existing rechargeable lithium-solid polymer electrolyte and lithium-liquid electrolyte batteries are also investigated. Part of this research is carried out under a Cooperative Research and Development Agreement with a commercial firm. Joint research is also conducted with the University of Minnesota Corrosion Research Center on the possible use of $\mathrm{V}_{2} \mathrm{O}_{5}$ films prepared by sol-gel methods as the cathodes for thin-film rechargeable lithium batteries and with the Electro-Optics Technology Center of Tufts University for the possible use of a carbon anode in thin-film lithium batteries.

\section{Sandia National Laboratories, New Mexico Albuquerque, NM 87185}

\section{Exploratory Batteries Department}

\section{Doping of Lithium Manganese Oxide for Improved Battery Performance Doughty, D.H. 505-845-8105 \\ $\$ 250,000$}

Cathode performance is critical to overall lithium ion rechargeable battery performance and impacts some of the most important challenges in the development of commercial lithium ion rechargeable batteries; namely energy density, cycle life, and rate capability. The influence of doping lithium manganese oxide cathode materials are being investigated on the fundamental properties that control cathode performance. The goal of the studies is to 
develop an improvied understanding of the chemical and physical properties that dètermine the performance of manganese oxide cathodes, as well as to produce improved materials and preparation techniques for cathodes. An atomistic model of structures of various manganese oxide and lithium manganese oxide compounds has been developed. The crystal structure of ramsdellite ' $\left(\gamma-\mathrm{MnO}_{2}\right)$, $\mathrm{LiMn}{ }_{2} \mathrm{O}_{4}$ spinel, and a lithium-deficient spinel $\mathrm{Li} \mathrm{i}_{1.0} \mathrm{M}_{2} \dot{\mathrm{O}}_{4}$ $\left(\lambda-\mathrm{MnO}_{2}\right)$ has been examined. Results from rigid ion and shelf models allow a systematic analysis of changes in lattice energy, cell volùme, strain, and the relative stability of doped structures úsing ions such as aluminum, cobalt, nickel, and titanium. The predictions from these calculations are guiding our synthesis effort. A nonaqueous coprecipitation process has been developed to prepare controlled stoichiometry lithium manganese oxalate precipitates that, when calcined, form high purity $\mathrm{Li}_{\mathrm{x}} \mathrm{Mn}_{\mathrm{y}} \mathrm{O}_{\mathrm{z}}$ powders. The resulting precipitated oxalates are readily converted to a number of phase pure lithium manganese oxides at moderate temperatures $\left(\leq 600^{\circ} \mathrm{C}\right)$ with the phase formed being dependent on the initial $\mathrm{Li} / \mathrm{Mn}$ ratio in the starting solution. The results of cathode performance tests of these materials are being studied. 


\section{OFFSITE INSTITUTIONS}

\section{Photochemical and Radiation Sciences}

\section{University of Akron Akron, OH 44325}

\section{Department of Chemistry}

\section{Dynamics of Charge-Transfer Excited States Relevant to Photochemical Energy Conversion \\ Lim, E.C. \\ 216-972-5297

The primary objective of the research is to gain a fundamental understanding of the factors governing the efficiency of charge transfer processes in molecular systems of interest, or pertinence, to photochemical energy conversion. The major focus of the current study is on the excited-state intermolecular interactions between two moieties that are brought together in close proximity, either by a short covalent linkage or by ground-state intermolecular association. Excited-state interactions ranging from strong charge transfer to weak van der Waals forces are being investigated for covalently bonded diaryl compounds in solution and for van der Waals complexes in supersonic free jets, using laser-based techniques. Where appropriate, and feasible, quantum chemical methods are also used to gain theoretical understanding of the energetics of charge transfer processes.

\section{University of Alabama Tuscaloosa, AL 35487}

\section{Department of Chemistry}

\section{Magnetic Resonance and Optical Spectroscopic Studies of Carotenoids Kispert, L.D. 205-348-7134

The current goal is to evaluate the role of polar media in the mechanism of carotenoid cation radical formation and decay and to determine the special properties of carotenoids, bound to pigment-protein complexes in photosynthetic membranes that enable them to serve both as antennae and as photoprotective agents and as a possible component of electron transfer processes. Simultaneous electrochemical and electron spin resonance measurements, simultaneous electrochemical and optical measurements, and simultaneous electrochemical and resonant Raman measurements have been carried out. From these studies, the reason has been deduced for the observation of carotenoid radicals in some photosystems and not others. In the solid state, the energy of the cis isomers falls close enough to that of the all trans isomers that the solid host can stabilize higher energy cis isomers. All trans or cisoidal carotenoid cation radicals can exist on solid supports and possibly in solution. The reason for the preference of the higher energetic twisted solid state configurations of the carotenoids in reaction centers has been determined. Semiempirical molecular orbital (RHF-INDO/ SP) calculations of the canthaxanthin cation radical in solution are in excellent agreement with the electron nuclear double resonance measurements. The host matrix is being manipulated in such a manner as to understand the carotenoid function and to ultimately develop predictive mechanisms for directing the outcome of photochemical events.

\section{Arizona State University Tempe, AZ 85287}

\section{Department of Chemistry}

\section{Supramolecular Structures for Photochemical Energy Conversion Gust, J.D., Jr.; Moore, T.A.; Moore, A.L. $602-965-4547$}

Photosynthetic solar energy conversion is the ultimate energy source for essentially, all life and is one of the most durable and efficient solar conversion "technologies." The goal of this project is to synthesize artificial photosynthetic reaction centers that employ the basic chemistry and physics of photosynthesis to help meet energy needs. Specifically, the research involves the preparation and study of photochemically active multicomponent molecules that functionally mimic photosynthetic light harvesting, photoprotection from light-initiated singlet .oxygen damage, and, most importantly, photoinduced multistep electron transfer to generate long-lived chargeseparated states with a quantum yield close to unity. One current project involves the preparation of molecular triads and tetrads featuring new linkages between the donor and acceptor moieties that lead to ultrafast electron transfer both in fluid solution and in glassy solids at low temperature. Another investigation deals with new methods for the stabilization of charge separation through intramolecular proton transfer.

\section{Boston University Boston, MA 02215}

\section{Department of Chemistry}

\section{Study of Intermediates from Transition Metal Excited-State Electron-Transfer Reactions \\ Hoffman, M.Z. 617-353-2494

The electron-transfer quenching of the excited states of :transition metal coordination complexes results in their 
one-electron oxidation or reduction, generating complexes with the metal center in unusual oxidation states or containing coordinated radicals, and the corresponding reduction of oxidation of the quencher. The major objective of this research, which utilizes the techniques of timeresolved spectrofluorimetry, cyclic voltammetry, and continuous and pulsed photolysis and radiolysis, is to gain an understanding of the parameters that govern the efficiencies of formation of energy carriers from the quenching reactions. Of particular concern have been the dependencies of the kinetics and efficiencies of all the mechanistic steps of the overall redox reaction on the temperature and the nature of the solution medium; these investigations demand, as well, that the properties and reactivities of the excited states of the photosensitizers and the reduced and oxidized species be fully understood. During the past year, the following projects have been investigated: (1) the specific effect of electrolytes on intermolecular electrontransfer reactions of excited $\mathrm{Ru}(\mathrm{II})$ diimine complexes; (2) the acid-base and electron-transfer properties of reduced $\mathrm{Ru}$ (II) complexes; (3) the electrochemical properties of organic quenchers and electron-transfer relays; (4) the photochemistry and photophysics of Cr(III)-polypyridyl complexes; (5) ground- and excited-state interactions of Ru(II) complexes with "innocent" solutes; and (6) the photosensitized oxidation of phenols via electron transfer and singlet oxygen mediation.

\section{Photoinduced Electron Transfer in Ordered Polymers \\ Jones, $G$. 617-353-2498}

Investigations involve the design and characterization of systems capable of photochemical electron transfer between electron donor and acceptor groups that are bound to polymer or biopolymer chains. Objectives of the research include the observation of the effects of polymer, peptide, or protein microenvironments on the efficiency and rate of electron transfer between groups that are separated by a number of chemical bond lengths. For these systems the (bio)polymer acts as a template or scaffolding for assembly of chromophores and photoactive species. Of special interest is charge migration among groups located at the ends of short peptide chains or along the axis of an [alpha] helix. Systems that have been prepared include diand tripeptides that have been modified by attachment of pyrene and xanthene dye chromophores and other photoactive groups. A family of new chromophores based on the acridinium ion has been synthesized. On photoexcitation these structures engage in intramolecular charge separation and give rise to chemically reactive radical pair states within picoseconds. Decay of the electron transfer intermediates (the time scale for molecular "switching") is variable over at least four orders of magnitude, reaching the 10-nanosecond time domain. Current work also includes the synthesis of amphipathic helices that will potentially provide protein "bundles" for assembly of synthetic reaction centers. Methods that are employed include peptide synthesis, laser flash photolysis and fast kinetics, fluorescence probes of polymer microdomains, circular dichroism, and molecular modeling. In these studies, emphasis is placed on the opportunities for construction of highly functionalized synthetic polymer materials in which reactive groups are held in relatively rigid arrays that provide controls at the molecular level of charge separation and photochemical energy storage. The work, is important to the understanding of charge transport in both natural and biomimetic systems and the development of energy conversion devices based on reversible electron transfer.

\section{Brandeis University Waltham, MA 02254}

\section{Department of Chemistry}

\section{Mechanistic Studies of Excited State Chemical Reactions \\ Linschitz, $H$. 617-736-2506}

Excited state redox reactions in homogeneous solution are being studied using nanosecond flash photolysis, to clarify the factors controlling rates and bulk radical yields. Test systems include prophyrins and fullerenes such as $\mathrm{C}_{60}$. The latter compound is especially suitable for mechanistic studies by virtue of its broad absorption, molecular symmetry and accurately known redox potentials and spectra of triplet and radical states. Of particular interest is the phenomenon of electron transfer within a redox couple concerted with proton displacements involving another species. This has been demonstrated in several hydrogenbonded systems. Such structurally imposed proton coupling provides means for controlling both the free energies and reorganization energies of endergonic photo processes, as well as the rates of dissipative back reactions that involve heavy particle movement. Hydrogen bonding and/or protonation also modifies the character of the substrate excited states. This is being studied in porphyrins by optical and spectroscopic titrations. In the case of mesotetraanilinoporphyrin, this leads to new types of charge transfer excited states with new photo-redox properties.

\section{California Institute of Technology Pasadena, CA 91125}

\section{Department of Chemistry}

\section{Picosecond Dynamic Studies of Electron Transfer Rates at III-V Semiconductor/Liquid Interfaces Lewis, N.S. 818-395-6335

Work in this laboratory is focused on elucidating the processes involved in charge transfer in photoelectrochemical solar cells. An understanding of these processes is important in solar energy conversion devices, the study of corrosion at semiconductor surfaces, and in further development of the theories of electron transfer at solid-liquid interfaces. The goal of this project is to use time-resolved measurements to obtain the desired kinetic information on these energy conversion systems. The semiconductor liquid junction is treated as a donor-acceptor system in which charge transfer to an acceptor is probed by observing the time-resolved decay kinetics of the initial photogenerated electron. In the specific case being studied in this laboratory, the "donor" is an epitaxial layer of GaAs, and the acceptor is the selenide ion in aqueous $1 \mathrm{M}$ 
KOH. Excess holes and electrons are created by a picosecond light pulse and then recombine by various pathways, including the one of primary interest-interfacial charge transfer. The entire kinetic scheme is monitored by the decay of light emission from the semiconductor. A computer model is then used to extract values for the heterogeneous charge transfer rates. GaAs surfaces treated with complexes of ruthenium, cobalt, and osmium have been found to yield faster luminescence decay rates than unmodified samples in contact with the same electrolyte. This corresponds to faster charge transfer from the semiconductor to the redox species and is in agreement with earlier work done in this laboratory. Modeling work is now being performed to more quantitatively describe these results in terms of specific heterogeneous electron transfer rate constants. While it might be expected that these rates could also be obtained from measurements of light-induced current decays, the effects of $\mathrm{RC}$ shaping on these electrical signals must be considered. Work has been completed on semiconductor liquid systems, demonstrating that the form of these decays is not sensitive to the interfacial kinetics and it can be adequately predicted from equilibrium properties of the semiconductor and solution.

\section{University of California, Berkeley Berkeley, CA 94720}

\section{Department of Chemistry}

\section{Theoretical Studies of Electron Transfer in the Photosynthetic Reaction Center Chandler, $D$. 510-643-6821

The structure, dynamics, and free energies pertaining to electron-transfer in complex systems are analyzed through large-scale numerical simulations and through analytical methods. The research on electron transfer is concerned with (1) the mechanism of charge transfer in photosynthetic systems; (2) derivation of analytical theories of electrostatics and solvation, tested by numerical simulation and used to explain measured free energetics for electron-transfer reactions; and (3) derivation of simplified quantum dynamical theories for electron transfer processes. These dynamical theories will be used to interpret and guide current simulation studies and to suggest new experimental work.

\section{Colorado State University Fort Collins, CO 80523}

\section{Department of Chemistry}

\section{Photoinduced Charge Separation in Linked Donor-Acceptor-Chromophore Systems \\ Elliott, C.M. \\ 303-491-5204

The primary goal of this research is to gain a better fundamental understanding of electron- and energy-transfer process. In photosynthesis light energy is converted ultimately to chemical energy. Nature has developed an elegant electron-transfer network that is superbly efficient at converting captured photons into separated charge. Abiological systems are capable of harvesting photons and separating charge as well. Frequently, however, the fate of this separated charge is geminate recombination rather than the production of useful chemical products. Our approach is to use synthetic chemistry to prepare complex assemblies that can be used to study intramolecular light-induced reactions. These assemblies will undergo intramolecular electron- and energy-transfer reactions with good quantum efficiencies, often producing long-lived charge separated states. The chemical systems under study are of two general types: ones that consist of a light-harvesting chromophore linked by conformationally flexible linkages to organic donor or acceptor moieties, and ones that consist of a metal-complex chromophore rigidly linked to a second metal complex that acts as the donor/acceptor. Through the design and study of such assemblies, we strive to gain a molecular-level understanding of the processes that will aid in the development of practical systems for conversion of light into chemically useful products.

\section{In situ Scanning Tunneling Microscopy Imaging of Dye Sensitization Processes at Layered Chalcogenide Semiconductor Electrodes Parkinson, B.A. 303-491-0540

Dye sensitization has the potential to increase the light utilization of large band gap semiconductors. The excited state of a dye molecule adsorbed onto the surface of the semiconductor electrode can inject electrons into the conduction band of an n-type semiconductor. These electrons can then be detected as a photocurrent generated at photon energies less than the band gap of the semiconductor. The quantum yield for electrons collected per photon absorbed by the dye at single crystal oxide electrodes was always less than a few percent. The quantum yield per absorbed photon can approach $100 \%$ when two dimensional chalcogenides are used as photoelectrodes. Recently methods for increasing the surface area of $\mathrm{SnS}_{2}$ photoelectrodes have been developed. By photoelectrochemically etching the surface in either acid or basic solutions, increases in the quantum yield for electron flow per incident photon have been obtained. The adsorption isotherm for methylene blue on etched and unetched surfaces has the same shape but shows an increase of 20 times more in the quantum yield. An in situ scanning tunneling microscopy method is being developed for detecting the position and energy levels of dye molecules adsorbed on these surfaces with molecular resolution. This is accomplished by modulating a light source at the wavelength of the dye absorption maximum and extracting the photoinduced contribution to the tunneling current via a lock-in amplifier. A simultaneous picture of photocurrent response and topography can then be obtained. Questions such as the state of dye aggregation on the surface and whether dye molecules are adsorbed on special sites could then be answered. The photo-STM technique has already been applied to semiconductor surfaces with bandgap light. New crystal growth techniques and new sensitization substrates are being developed in order to increase the efficiency and expand the spectral range of sensitization. 


\section{University of Colorado Boulder, CO 80309}

\section{Department of Chemistry and Biochemistry}

\section{Mechanistic Aspects of Photoconversion at Semiconductor-Liquid Junctions and in Facilitated Transport Membranes Koval, C.A. 303-492-5564 \\ $\$ 100,000$}

The goal of this research is understanding how solar energy can be utilized in photoelectrochemical cells and facilitated transport membranes. Reactions of "hot" electrons that are created by light absorption in a semiconductor electrode have the potential to increase the energy conversion efficiency or to alter the product distribution in photoelectrochemical cells. Current investigations involve studying the reduction of a variety of organobromide compounds by electrochemical techniques and by single electron reducing agents. These studies will lead to a variety of redox systems that can be used to detect hot electrons reactions at semiconductor electrodes. Rotating ring disk electrochemical methods are being adapted to study the wavelength dependence of photoreduction due to size quantization at superlattice electrodes. Photochemistry in membranes can allow chemical species to be transported against their concentration gradients. In principle, this process can be used in a variety of contexts ranging from environmental restoration to energy storage. Liquid and polymer membranes that contain photochemically active carriers are prepared and characterized. Mlumination of these membranes allows ions or molecules to be selectively separated and concentrated. A mathematical model based on molecular parameters (reaction rates, excited state lifetimes, interfacial kinetics, etc.) that describes this type of membrane process is being developed. This model will be used to guide subsequent experiments aimed at improving the selectivity, productivity, and photoefficiency of photochemically controlled membrane transport.

\section{Columbia University New York, NY 10027}

\section{Department of Chemistry}

\section{Charge Generation and Separation at Liquid Interfaces \\ Eisenthal, $K . B$. 212-854-3175 \\ $\$ 120,000$}

Polarity is a key factor in determining chemical equilibria and kinetics of chemical reactions at interfaces, especially reactions involving charge transfer processes. We are developing an interfacial polarity scale based on measurements of the wavelength-dependent second harmonic signals at liquid interfaces. The molecules selected undergo charge transfer transitions, one shifting to the red and the other to the blue as polarity changes. Initial results indicate that the polarity at the air/water interface is less than in bulk water, corresponding to a polarity between bulk proprionitrile and bulk butanol. Femtosecond pump-second harmonic probe experiments have detected ultrafast energy dissipation by photoexcited molecules at aqueous interfaces. The conversion of solute electronic energy to the thermal energy (librational and translational) of surrounding water molecules can raise the local temperature several hundred degrees. The adsorbed molecules rapidly reorient and leave the interface region resulting in the observed decrease in the second harmonic signal within 10 picoseconds of the photoexcitation, the decrease depending on the pump energy. In other experiments, we have found that it takes roughly 10 seconds for the interface to return to equilibrium. Picosecond sum frequency experiments on a series of linear nitriles have yielded the significant result that a density of $\sim 4 \times 10^{14}$ nitriles $/ \mathrm{cm}^{2}$ at the air/nitrile-water interface is required to trigger a structural phase transition.

\section{Theoretical Studies of Electron Transfer and Optical Spectroscopy \\ Friesner, R.A. 212-854-7606

This project involves theoretical studies of small semiconductor clusters and other complex systems of interest in solar photochemistry. An empirical pseudopotential model has been used to calculate bandgaps in small semiconductor particles to within $\sim 0.1 \mathrm{eV}$ as a function of particle size for three materials: $\mathrm{CdS}, \mathrm{CdSe}, \mathrm{Si}$. Calculations of the absorption spectrum and the homogeneous zero phonon linewidth for CdSe particles, the result for which can be compared with experiments, are currently in progress. Preliminary results computing eigen states and energies via the Lanczos algorithm have been obtained, a necessary precondition for dynamical simulations. Methods have been developed for quantum dynamical calculations on complex systems and applied to electron transfer through DNA. Methodology to derive the appropriate Redfield equations from a microscopic Hamiltonian using canonical transformations to render the perturbatively treated part of the Hamiltonian small is in progress. Simulations of electron transfer in DNA predict that longrange transfer should be quenched at low temperature.

\section{Photo-CIDNP of Photosynthetic Reaction Centers \\ McDermott, A.E. 212-854-8393

The Chemically Induced Dynamic Nuclear Polarization (CIDNP) process is used to observe the nuclear magnetic resonance (NMR) spectra of ${ }^{15} \mathrm{~N}$-labeled photosynthetic reaction centers. In simple echo-detected Bloch decay spectra collected under magic-angle spinning with or without proton decoupling, nuclear polarization has been detected for the nitrogens in the tetrapyrroles of the bacteriochlorophyll special pair ("P"), associated imidazoles, and the primary acceptor pheophytin ("I") that are far from Bolzmann equilibrium. The resulting NMR lines are emissive and 300 times the intensity of the thermally relaxed nuclei. The polarization is not observed if the quinones are present and preoxidized. The signals presumably result from a transient nonequilibrium mixing of the singlet and triplet states of the initially formed charge transfer pair, $\mathrm{P}^{+} \overline{\mathrm{I}}^{-}$. Selectively labelled samples have been used to assign the signals, and ongoing efforts are aimed at extensions to the plant reaction centers. 
Department of Electrical Engineering

\section{Translational-Energy-Resolved Studies of Photogenerated Carrier-Induced Reactions on UHV Semiconductor Surfaces \\ Osgood, R,M, Jr. \\ $212-854-4462$}

Photodissociation of methyl halides $\left(\mathrm{CH}_{3} \mathrm{X}, \mathrm{X}=\mathrm{Br}, \mathrm{Cl}, \mathrm{I}\right)$ on GaAs (110) using time-of-flight (TOF), temperature programmed desorption (TPD), and photoluminescence (PL) in ultrahigh vacuum (UHV) has revealed the operation of several photochemical processes on semiconductor surfaces, For example, photogenerated substrate electronhole pairs promote adsorbate dịssociation via an electron attachment mechanism to form a negative ion resonance with subsequent cleavage of the $\mathrm{C}-\mathrm{X}$ bond. For the case of $\mathrm{CH}_{3} \mathrm{Br}$, dissociative attachment of electrons at the conduction band minimum (CBM) or hot, photoemitted electrons is observed to result in distinct kinetic energies for the ejected methyl fragments. Wavelength variation indicates a threshold at the band gap for the first mechanism and $3.5 \mathrm{eV}$ in the second. The CBM process exhibits efficient self-quenching behavior due to electron-hole recombination via Br-induced surface states produced as a result of the photodissociation process itself. This hypothesis is supported by PL measurements, in which the PL yield exhibits a parallel decrease upon adsorbate photodissociation. Angular variation of the photoyield suggests that the adsorbate adopts a strongly tilted geometry with respect to the surface normal-a hypothesis confirmed by a 'direct' measurement of the adsorbate geometry via the NEXAFS technique and by modeling of the magnitude of the adsorbate-adsorbate repulsive interaction manifested in TPD spectra. Also, recent theoretical modeling by our group using ab initio calculations have also confirmed this geometry for the case of $\mathrm{CH}_{3} \mathrm{Br}$ interacting with a $\mathrm{GaAs}$ cluster, Our most recent experiments have applied this experimental approach to an investigation of the more complex dissociation dynamics of methylated metalorganic chemical molecules $\left[\left(\mathrm{CH}_{3}\right)_{2} \mathrm{X}, \mathrm{X}=\mathrm{Zn}, \mathrm{Cd}\right]$ on model III-V semiconductor surfaces. While complex dissociative thermal chemistry is found to dominate the adsorption process on GaAs surfaces, molecular adsorption/ desorption phenomena are the dominant thermal processes for the case of adsorption on the $\mathrm{CdTe}(110)$ surface. In this system, photodissociation of the molecular adsorbate with TOF analysis of the resulting photofragments again reveals the existence of multiple channels for photodissociation with distinct thresholds and photofragment kinetic energies. While one observed channel clearly relates to the direct photon absorption process, the striking coverage dependence of the second channel again suggests a surface mediated process is operative. Current research is aimed at gaining detailed insight into the microscopic mechanism underlying this reaction channel.
Dartmouth College

Hanover, NH 03755

Department of Chemistry

\section{Photoinduced Dipoles and Charge Pairs in Condensed Phase \\ Braun, C.L: \\ 603-646-2500 \\ $\$ 100,000$}

The objective is to understand photoionization in liquids and solids comprising organic molecules. One goal is to understand the formation and recombination or separation of hole-electron pairs formed by absorption of visible photons in electron donor-acceptor materials. Fast current measurements are used to follow the separation of the resulting holes and electrons (or ions). The fast photocur: rent measurements led to measurement of the dipole moments of excited molecules in solution with the same techniques. The dipole moment technique has proved useful in the study of both intramolecular and intermolecular charge transfer. A dipole moment of $89 \mathrm{D}$ (corresponding to a charge separation of $1.8 \mathrm{~nm}$ ) has been measured for a donor-acceptor triad molecule, synthesized at Argonne National Laboratory. Charge separation of $0.7 \mathrm{~nm}$ is seen in the triplet exciplex formed by $\mathrm{C}_{60}$ and a substituted benzene donor. While in the $\mathrm{C}_{60}$ exciplex the charge transfer is complete; in many other exciplexes it is not. Smaller dipoles and partial charge transfers are being studied in weaker donor-acceptor pairs.

\section{Georgia Institute of Technology Atlanta, GA 30332}

\section{School of Chemistry and Biochemistry}

\section{Time-Resolved Laser Studies of the Proton Pump Mechanism of Bacteriorhodopsin El-Sayed, M.A. 310-825-1352 $\$ 135,000$}

There are two basic systems in nature that convert solar energy into chemical energy, i.e., undergo photosynthesis. The first is the chlorophyll-based system present in green plants and the other is bacteriorhodopsin $(\mathrm{bR})$ present in Halobacterium Salinarium. In both systems, solar energy is first converted into electric energy and then into chemical energy stored in the chemical bonds of adenosine triphosphate (ATP). The final step in the solar to electric energy conversion involyes the formation of proton gradients. In both systems, the mechanism of the conversion of the proton gradients into ATP is the same while the molecular mechanism of the conversion of the solar energy into proton gradient is very different. In chlorophyll, it involves electron pumps while in $b R$ the absorption of light leads to very rapid (450 femtosecond) retinal isomerization, separation of positive and negative charges, and protein conformation changes that finally lead to pumping protons from inside the cell to the membrane surface, thus creating the proton gradients; thus bR is a solar proton pump. This pump requires metal cations for its function. Our present research is focused on trying to answer two fundamental questions regarding the proton pump: (1) What are the molecular mechanisms by which the protein catalyzes the retinal photoisomerization in $\mathrm{Br}$ ? and (2) 
What role do metal cations play in the proton pump? We are presently determining the femtosecond photoisomerization time of retinal and its modified derivatives in $b R$ and some of its mutants in which charged and hydrogen bonding residues in the retinal cavity are individually replaced by neutral nonhydrogen bonding ones. The observed results are examined in terms of the electronic and steric effects on the retinal excited state potential energy surface in $\mathrm{bR}$. Theoretical simulation studies are being carried out to test the effect of the charge distribution around the retinal as well as the steric factors of the side groups of the surrounding residues. In order to understand the role of metal cations in the bR function, their location must first be determined. In this effort, their binding constants have been determined in $b R$ and in a number of its mutants. The two high affinity cations, one of which is vital to the function, are electrostatically coupled to the charged residues within the retinal cavity. Now attempts are made to locate the position of these two metal cations by use of anomalous X-ray and extended X-ray absorption fine structure techniques using the Synchrotron Radiation Source at Brookhaven National Laboratory.

\section{University of Houston Houston, TX 77204}

\section{Department of Chemistry}

\section{Charge Separation in Photoredox Reactions \\ Kevan, $L$. 713-743-3250}

This research is directed toward a better molecular understanding of the structural aspects controlling charge separation in photoredox reactions in organized molecular assemblies with emphasis on vesicles and microporous oxides. Control of the location of an electron donor will be achieved by attachment of variable length alkyl chains to synthesized porphyrins. Control of the electron acceptor location is also being initiated. Photoyields will be monitored by electron spin resonance and the photoproduced cation location will be assessed by deuterium electron spin echo modulation and matrix proton electron nuclear double resonance. The results will help determine the structural requirements for optimizing photoinduced charged separation for the storage of light energy

\section{Marquette University Milwaukee, WI 53233}

\section{Department of Chemistry}

\section{Photophysical and Photoredox Studies of Transition Metal Complexes and Zeolite-Entrapped Assemblies Kincaid, J.R. 414-288-3539

The general goals of this research program are to develop a reliable interpretive framework for analysis of the resonance Raman and time-resolved resonance Raman spectra of potential photosensitizers based on transitionmetal complexes of polypyridines and phthalocyanines and related ligands and to apply these techniques to elucidate the photophysical and photoredox properties of such species both in solution and in various organized assemblies. Recent detailed studies of the temperaturedependent lifetimes of a series of heteroleptic complexes have helped reveal the interplay of two thermally activated decay pathways from the lowest excited state. Work continues on the synthesis and characterization of zeolite entrapped polypyridine complexes of ruthenium and recent work documents dramatic increases in ${ }^{3}$ MLCT state lifetimes brought about by the steric constraints of the $\mathrm{Y}$ zeolite supercage. Synthetic methods are being developed to permit construction of organized molecular assemblies within the zeolite framework, and preliminary photophysical measurements document an interaction of the assembled components. Such systems may represent an effective strategy to achieve significant gains in net charge-separation upon photoexcitation.

\section{Department of Chemistry}

\section{Magnetic Resonance Studies of Photoinduced Electron Transfer in Homogeneous and Heterogeneous Media van Willigen, $H$. 617-287-6147 \\ $\$ 110,000$}

This . research is concerned with the application of time-resolved Electron Paramagnetic Resonance (EPR) techniques in the study of photoinduced electron transfer reactions. The application of these techniques in this area of research is of interest for several reasons. First, high spectral resolution makes it possible to identify paramagnetic molecules and to obtain information on their interaction with the environment. Second, the time development of the spectra generally is affected by chemically induced dynamic electron polarization (CIDEP). Identification of CIDEP effects provides unique mechanistic insights. Third, relaxation data can be used to study the effect of temperature and medium on molecular motion. A Fourier transform EPR study was made of oxidative and reductive electron transfer quenching of $\mathrm{C}_{60}$ triplets $\left({ }^{3} \mathrm{C}_{60}\right)$ and of photochemical reactions in which $\mathrm{C}_{60}$ acts as a light harvester and photosensitizer. Studies are made as well of the photophysics and photochemistry of ${ }^{3} \mathrm{C}_{60}$ in polymer films. Finally, the possibility of using EPR to study the mechanism and kinetics of photochemical at the surface of semiconductor particles is being explored. 


\section{University of Minnesota Minneapolis, MN 55455}

\author{
Department of Chemistry
}

\section{Femtosecond Time-Resolved Experiments on the Solvated Electron and Intermolecular Charge Transfer in Solution}

Barbara, P.F. 612-625-0064

$\$ 170,000$

A new experimental approach to the investigation of the photophysics of the solvated electron has been developed. The technique involves three separate laser pulses. The first pulse generates solvated electrons, the second pulse excites the electron from the ground state to the first excited state, and the third pulse probes the dynamics of the ground state repopulation and the ground and excited state relaxation. The electron has been studied in water and a variety of alcohols on the femtosecond time scale. In addition to timeresolved measurements, transient polarization anisotropy measurements have been made. The results are in excellent agreement with nonadiabatic molecular dynamics simulations of the solvated electron leading to a detailed understanding of the photophysics, structure, and dynamies of this central species in radiation chemistry. In addition to studies on the solvated electron, the photophysics of the charge transfer band of the $\mathrm{Br}$ atom/benzene charge transfer complex have been made in order to understand the ultrafast electron transfer of this complex.

\section{The Contribution of Electronically Excited States to the Radiation Chemistry of Organic Systems \\ Lipsky, $S$. 612-624-9581 \\ $\$ 105,000$}

Photophysical properties of simple organic liquids that have relevance in determining their behavior when subjected to high-energy radiation are being studied. Recent work includes (1) determination of the ionization threshold of neat liquid benzene and estimation of its ionization efficiency and electron range distribution function from dependence of photocurrent on photon energy and on strength of applied electric fields; (2) determination of the ionization thresholds of some saturated hydrocarbon liquids and estimation of their electron range distribution functions from the effect of a magnetic field to enhance the recombination fluorescence of dissolved low concentrations of aromatic molecules or, in some cases, to effect the recombination fluorescence of the neat liquid itself; and (3) measurement of the fluorescence action spectrum of benzene as a function of benzene concentration in various saturated hydrocarbon and perfluorocarbon solvents over an excitation energy interval from $120 \mathrm{~nm}$ to $250 \mathrm{~nm}$. This work is designed to characterize the "channel 3 " behavior in the condensed phase and to unravel peculiar effects of increasing benzene concentration to inhibit "channel 3 " in certain spectral regions.

\section{National Institute of Standards and Technology, Gaithersburg Gaithersburg, MD 20899}

\section{Chemical Kinetics and Thermodynamics Division}

\section{Electron Transfer Reations of Metalloporphyrins \\ Neta, $P$.} 301-975-5635

The pulse radiolysis technique is applied to the study of electron transfer and other processes involving reactive intermediates. These studies are concerned with mechanisms, kinetics, and thermodynamics of reactions of organic and inorganic radicals and unstable oxidation states of metal ions. The studies focus on the unique ability of pulse radiolysis to provide absolute rate constants for reactions of many inorganic radicals and organic peroxyl radicals, species that are key intermediates in many chemical processes. Recent measurements have focused on solvent effects on the rate constants for reactions of peroxyl radicals with several compounds, including porphyrins, and on one-electron oxidation of porphyrins by other species. Peroxyl radicals derived from halogenated aromatics are currently studied. A special concern of this work is the study of electron transfer reactions of porphyrins and metalloporphyrins, which permits evaluation of these molecules as intermediates in solar energy conversion. Metalloporphyrins react with free radicals via electron transfer, involving the ligand or the metal center, or via bonding to the metal. Currently under study is the reduction of rhodium porphyrins and the possibility of carbon dioxide reduction or insertion into alkyl-metal bonds.

\section{University of New Orleans New Orleans, LA 70148}

\section{Department of Chemistry}

\section{Electronic and Nuclear Factors in Intramolecular Charge and Excitation Transfer \\ Piotrowiak, $P$. 504-286-6840 \\ $\$ 85,000$}

The project addresses two aspects of photoexcited charge separation and recombination processes: (1) the role of the molecular orbital and vibrational mode symmetry in determining the electronic coupling between the donor and the acceptor, and (2) the role of ion pairing and ionic atmosphere relaxation in controlling the efficiency and lifetime of intramolecular charge separation. After demonstrating the strong vibronic state specificity of the electronic coupling and excitation transfer in rigid spiranes, the work in the first area concentrates on experiments probing the influence of state density on the effective donor-acceptor coupling. A new series of nondegenerate spiranes with varied $\Delta \nu=0$ energy gaps is being investigated. Double photon ionization experiments on the same systems are also in progress. In the second area, it has been shown that transient triplet charge transfer absorption bands can serve as highly sensitive probes of ion pairing dynamics and energetics. Variable temperature 
measurements of the activation energy for the association between the photoinduced charge-separated species and ions in solution are in progress. An important model compound with an ion permanently tethered to the donoriacceptor molecule has been synthesized. This system will allow investigation of intramolecular ion association processes, in which the random diffusion has been eliminated.

\section{University of North Cärölinà at Chapel Hill Chapel Hill, NC 27599}

\section{Department of Chemistry}

\section{Excited Státe Prôcesses in Transition Metal Complexes. Redox Splitting in Soluble Polymers \\ Meyer, T.J. \\ 9.19-962-6319 \\ $\$ 155,000$}

Polypyridyl complexes of $\mathrm{Ru}(\mathrm{II}), \mathrm{Os}(\mathrm{II})$, and $\mathrm{Re}(\mathrm{I})$ are being investigated for applications in molecular-level energy conversion processes. The photochemical and photophysical properties of these complexes are well understood and provide a basis for predicting light absorption and excited state stabilities and lifetimes. Based on this knowledge, synthetic procedures are being developed for the preparation of a new family of black absorbers that absorb light efficiently throughout the near-UV visible. These molecules emit in the near infrared, are photochemically stable, and have excited states whose lifetimes are suffciently long to be accessible. In this class of complexes, emission and resonance Raman spectroscopies are being used to explore the roles of electronic structure, molecular vibrations, 'and the medium on properties and lifetimes. Synthetic methods have been developed based on 'ether 'or amide links for attaching derivatives of these complexes to soluble polymers along with organic groups that'can act as electron or energy transfer donors or acceptors. Photophysical studies on the resulting molecular assemblies show that soluble polymers can be designed in which photoinduced electron or energy transfer can be initiated and controlled on single polymeric strands. The goals of this work are to mimic the light harvesting, electron transfer abilities of the reaction center of photosynthesis and to study long-rảnge electron and energy transfer.

\section{Northwestern University Evanston, IL 60208}

\section{Department of Chemistry}

\section{Dynamic Structural Studies of} Light-Induced Charge'Tränsfer and Electronic Localization'Delocalization Phenomena in Metal-Based Molecular Systems

Hupp, J.T. 708-491-3504

This project involves (1) time-dependent scattering studies of vibrational structural changes accompanying chemically important photoredox processes; (2) collaborative studies of femtosecond charge-transfer kinetics in fully vibrationally characterized systems; (3) flow and laser studies of somewhat slower electron transfer in thermodynamically well-defined, ligand-bridged redox systems; and (4) resonant vibrational characterization of delocalized intervalence transitions. The combination of (2) and (3) has now yielded data that span more than 11 orders of magnitude in intramolecular electron transfer reaction rate. Current experiments at the rate extremes (i.e., ultrafast and moderately slow) are yielding important new information concerning the role of solvent and internal modes, including vibrational coherence in back electron transfer reactions and possible direct coupling of intermediate frequency solvent modes to the electron transfer reaction process. New results from area (4) are being used to explore the efficacy of primitive "molecular wires" in long-range charge separation.

\section{Vibrational Dynamics in Photoinduced Electron Transfer \\ Spears, K.G. $708-491-3095$

Theory and experiment suggest that molecular vibrations and distortions are important controlling elements for electron transfer. The objectives of the project are to develop a new molecular understanding of electron transfer processes. The unique method of picosecond infrared absorption spectroscopy is being used to monitor electron transfer kinetics. The first case of vibrational state dependent electron transfer has been experimentally determined. The electron transfer is between two cobalt metal atoms interacting in a solvent-caged, contact pair. The compound has a cobaltacinium cation and a cobalt tetracarbonyl anion with a visible absorbing chargetransfer band. The neutral pair created by a pulse of visible light has a rate of electron transfer that is dependent on vibrational quantum number, and is very sensitive to solvent polarity. The ion pair formed after the electron transfer has up to four'quanta of the $\mathrm{CO}$ stretch mode, and the energy relaxation rate was measured. New theoretical models have been developed that incorporate all the energy relaxation and electron transfer rate competitions.

\section{Ohio State University Columbus, OH 43210}

\section{Department of Chemistry}

\section{Photochemistry in Constrained Spaces: Zeolites and Layered Double Metal Hydroxides}

Dutta, $P: K$. 614-292-4532

Zeolites and layered double-metal hydroxides are being investigated as supports for photochemical redox assemblies. In the case of zeolites, efficient photoelectron transfer from intrazeolitic $\mathrm{Ru}(\mathrm{bpy})_{3}{ }^{2+}$ to a neutral viologen in solution is mediated by an intrazeolitic viologen in the absence of a sacrificial electron donor. 'The geometric constraints imposed by the zeolite on large intrazeolitic viologens like benzylviologen has been exploited to alter its reduction potential and increase the charge separation 
yield. The chemistry of intrazeolitic $\mathrm{Ru}(\mathrm{bpy})_{3}{ }^{3+}$ has been studied. This species is unstable in aqueous solution at neutral to basic $\mathrm{pH}$ because of intermolecular reactions leading to degradation. Because of the lack of translational mobility in the zeolites, the reaction of $\mathrm{Ru}(\mathrm{bpy})_{3}{ }^{3+}$ with water takes a different route. Hydroxide attack on the bipyridyl ligand is followed by release of hydroxyl radicals. This is evident from diffuse reflectance, resonance Raman, and EPR studies. By gas chromatographic methods, the evolution of $\mathrm{O}_{2}$ is observed; it is proposed that the hydroxyl radicals combine to form $\mathrm{H}_{2} \mathrm{O}_{2}$, which is then oxidized to $\mathrm{O}_{2}$. The rate of the overall reaction is enhanced in basic medium. In the area of layered double-metal hydroxides, the dynamics of the alkyl chains in the interlayer space have beed studied by infrared spectroscopy and pyrene fluorescence. $\mathrm{TiO}_{2}$ in these interlayers has been sensitized with porphyrins, and photoelectron transfer to viologens in solution has been observed in the presence of sacrificial electron donors.

\section{University of Oregon Eugene, OR 97403}

\section{Department of Chemistry}

\section{Photochemical Water-Splitting Using Organometallic Oxides as Sensitizers Tyler, D.R. 503-346-4649

The project objective is to split water photochemically into hydrogen and oxygen using homogeneous molybdenum oxide catalysts. The first catalyst being studied is molybdocene oxide. Irradiation of this molecule forms molybdocene and presumably, oxygen. (This latter point is still being investigated.) Results also show that the molybdocene generated in this reaction reacts with water to reform the oxide complex and hydrogen gas. The hydrogen is formed quantitatively. Efforts in the forthcoming year will focus on determining the fate of the oxygen in the irradiation of molybdocene oxide. Mechanistic studies of the hydrogen and oxygen producing reactions are underway. These studies will help to minimize the side reactions that occur in the oxygen-forming reaction. This system represents one of the first in which both hydrogen and oxygen are formed from the same catalyst in homogeneous solution.

\section{Pennsylvania State University, University Park University Park, PA 16802}

\section{Department of Chemistry}

\section{Electron Transfer Reactions in Microporous Solids Mallouk, T.E. 814-863-9637}

This project uses microporous solids and surface assemblies as organizing media for light-induced intermolecular electron and energy transfer reactions. The role of the solid is to control the juxtaposition of redox-active :and photoactive molecules in space, and also, in some cases, to participate as an active component of the electron or energy transfer cascade. One aspect of the project involves the sensitization of layered oxide semiconductors, such as alkali niobates and titanoniobates, with ruthenium polypyridyl photosensitizers. When loaded with interlamellar metal catalysts, these materials are catalysts for photogeneration of hydrogen from water and nonsacrificial electron donors, such as iodide. Factors that control the quantum efficiency of this process are being studied and optimized. A new technique has been devised for exfoliating these lamellar semiconductors and restacking them on high surface area substrates, with control over the stacking sequence at the monolayer level. This method is now being used to prepare multicomponent electron and energy transfer chains, in which individual electroactive and photon harvesting polymer monolayers are separated by single sheets of oxide semiconductors or insulating metal phosphates. The kinetics of electron transfer reactions in these assemblies are being studied by flash photolysis techniques. The role of oxide and chalcogenide semiconductors as superexchange mediators of molecular electron transfer reactions is also being studied.

\section{Polar Solvation, Dielectric Friction, and Electron Transfer Maroncelli, $M$. 814-865-0898 $\$ 112,000$}

The focus of this project is on understanding how polar solvents influence electron and other charge transfer reactions in solution. Of special interest are dynamical aspects of the solvation process and the role that such dynamics play in determining charge transfer rates. A number of recent theories have predicted a proportionality between the rate of electron transfer and.solvent reorganization rates. Time-resolved fluorescence studies on simple intramolecular charge transfer reactions are used to explore this connection. An important part of the work currently in progress also involves obtaining a prerequisite understanding of the dynamics of solvation in nonreactive systems. Results obtained to date indicate that the time scales of solvation in polar liquids are poorly predicted by simple continuum models of solvation, but it is just such models that have been used to theoretically study the connection between solvation, and electron transfer. In order to build a more adequate, understanding of charge transfer in solution it is first necessary to better understand and model the dynamics of solvation in simple, nonreactive situations. Time-resolved experiments and molecular dynamics computer simulations are being used to probe the dynamics of solvation in a variety of solvents and solvent mixtures. The goal of this work is to develop and test simple models of the static and dynamic aspects of polar solvation of relevance to the solvent-reaction coupling. 


\section{University of Pennsylvania Philadelphia, PA 19104}

\section{Department of Chemistry}

\section{Evaluation of Electronic Coupling In Photoinduced Electron Transfer Reactions Therien, M.J. 215-898-0087 $\$ 125,000$ (15 months)}

The primary objective of this project is to delineate the relative and absolute importance of the factors that determine the dimension of the electronic coupling matrix element $\left(\mathrm{H}_{\mathrm{ab}}\right)$ for photoinduced and thermal charge recombination electron transfer processes. These studies are being carried out with several families of donor-spaceracceptor (D-Sp-A) complexes that utilize electronically excited porphyrin donors and quinone acceptors. A key feature of this effort lies in the detailed focus on the role played by the tunneling medium in such reactions and the parameters that should affect the dimension of $\mathrm{H}_{\mathrm{ab}}$ such as absolute D-A energetics, medium topology, and medium electronic structure. Experimental work in the progress focuses on probing the nature of charge tunneling interference phenomena as well as how medium band energetics, $\sigma-\pi$ electronic coupling, $\pi$-manifold orientation, and medium excited electronic states establish the magnitude of $\mathrm{H}_{\mathrm{ab}}$. The fundamental information obtained in these studies will be useful in directing and controlling the electron and energy transfer processes essential to effective photoconversion.

\section{University of Pittsburgh Pittsburgh, PA 15260}

\section{Department of Chemistry}

\section{Time-Resolved Optical Studies of Charge Relaxation and Charge Transfer at Electrode Interfaces \\ Waldeck, D.H. 412-624-8430

The objective of this project is to develop a quantitative understanding of the kinetics of photogenerated carriers in semiconductor electrodes and at the semiconductor/ electrolyte interface. This goal is being pursued on three fronts. A computer algorithm is being developed to simulate the fluorescence decay of semiconductor band gap emission. Experimental studies are proceeding on the fluorescence decay of InP and its voltage dependence. Electrochemical photocapacitance studies of $\mathrm{TiO}_{2}$ electrodes that are chemically modified were recently completed. Time-resolved studies of the primary electron transfer step in this system are underway. Self-assembled monolayers of octadecylthiols on InP electrodes are being prepared for future studies.

\section{Portland State University Portland, OR 97207}

\section{Department of Chemistry}

\section{Asymmetric Polymeric Porphyrin Films for Solar Energy Conversion \\ Wamser, C.C. 503-725-4261

This project involves the synthesis and characterization of thin films of polymeric porphyrins, where such films are potentially useful as components of solar energy conversion methods. Two different approaches have been used to create polymeric films of substituted tetraphenylporphyrins on transparent electrodes: (a) interfacial polymerization of a pair of reactive monomers into a thin film, later deposited onto an electrode, or (b) oxidative electropolymerization of electron-rich porphyrins directly onto an electrode. For interfacial polymerization, an aqueous solution of either tetra(4-hydroxyphenyl)porphyrin (THPP) at $\mathrm{pH} 11$ or tetra(4-aminophenyl)porphyrin (TAPP) at $\mathrm{pH} 3$ is layered atop a dichloromethane solution of tetra(4-chlorocarbonylphenyl) porphyrin (TCCPP), creating a thin polyester or polyamide film at the interface. Such films are highly crosslinked, with a distinctive asymmetry of functional groups that creates a gradient of porphyrin redox potentials across the film. Observed photopotentials are directional, with the charge separation following the predicted direction of the redox potential gradient. Continued work in this area is aimed at characterizing the unique structural asymmetry and developing a model of the directional charge transport processes within the film. Electropolymerized films of TAPP are also being studied as possible conductive mediators for dyesensitized semiconductor solar cells.

\section{Princeton University Princeton, NJ 08544}

\section{Department of Chemistry}

\section{Resonance Raman Spectroscopy of Photoreaction Centers \\ Spiro, T.G. 609-258-3907}

Resonance Raman spectroscopic techniques are applied to investigation of vibrational spectra of the chromophores in the electron transfer chain of the reaction center of the photosynthetic bacterium Rhodobacter sphaeroides. Distinct differences have been detected between the spectra of the two bacteriopheophytin chromophores, one of which is on the electron transfer pathway and the other of which is not. The temperature variation of these spectra suggest that there are protein structure changes associated with the temperature variation of the electron transfer rate. The nature of the recently discovered low-frequency modes of the special pair is being investigated by using depolarization measurements, which may help to distinguish between intramolecular and intermolecular vibrations. Transient resonance Raman spectroscopy will be used to study the role of protein dynamics via the evolution of the vibrational spectra of the electron transfer intermediates. 


\section{University of Rochester Rochester, NY 14627}

\section{Department of Chemistry}

\section{Photochemistry of Dithiolate Complexes of the Platinum Group Elements Eisenberg, $R$. 716-275-5573

Diimine dithiolate complexes of platinum(II) and other $\mathrm{d}^{8}$ metal ions are being investigated for their potential use as photoreduction catalysts in the conversion of light-tochemical energy. Studies in previous years on Pt diimine dithiolate complexes have shown that these solution emissive compounds have a charge transfer excited state involving a $\pi$ \{sup*\} orbital of the diimine as the acceptor function and a mixed $\mathrm{Pt}(\mathrm{d}) / \mathrm{S}(\mathrm{p})$ orbital as the filled donor function. A systematic study of this chromophore reveals that the lifetime of the emissive state correlates with its energy in a manner characteristic of the energy gap law. A new set of complexes has also been synthesized in which the thiolate donors are connected to a redox active group-in this case, ferrocene-so that upon charge transfer excitation, the hole created at the sulfur donors is rapidly filled from the redox active moiety, leading to a more stable, charge separated state. Further elaboration of this structure to allow productive chemistry to emanate from the energetic electron transferred into $\pi^{*}$ dimine is in progress. Finally, new Schiff base complexes of rhodium have been synthesized and characterized regarding their electronic structures and reaction chemistry. Square pyrimidal Rh(III) complexes exhibit weak solution luminescence and undergo photolysis to generate metalloradical species that are important in substrate activation reactions.

\section{Femtosecond Studies of Semiconductor Photochemistry: Criteria for Hot Electron Energy Storage \\ Miller, R.J.D. \\ 716-275-4079

This work exploits semiconductor liquid junctions as an optical switch to turn on the surface photochemistry. These systems are extremely efficient at charge separation (photocurrent quantum yield $\sim 1$ ) and can exhibit solar energy conversion efficiencies approaching those of the best solid state devices. The most tantalizing feature of these systems is that theoretically they are capable of attaining nearly twice the solar energy conversion efficiencies of all solid state semiconductor systems. Due to the discrete nature of molecular acceptors, it is possible to avoid energy conversion losses in the junction through lattice heating. However, to achieve this goal, interfacial charge transfer must be made fast enough to compete with carrier thermalization. Whether or not this is possible is an important issue as it determines the level of theoretical modeling needed to properly treat the electronic coupling between the highly delocalized states of the solid state and the molecular acceptors across the interface. To this end, a number of nonlinear optical methods have been conducted to arrive at a real-time view of interfacial charge transfer processes at $\mathrm{GaAs}(100)$ / $\left(\mathrm{Se}^{-2 /-1}\right)$ aqueous liquid junctions. Studies of the interfacial hole transfer dynamics, field accelerated transport, and dynamic studies of the water response function have shown that interfacial charge transfer can occur near strong coupling limits. Based on studies of carrier relaxation in the bulk, these studies suggest that a significant fraction of carriers undergo interfacial transitions prior to thermalizing, i.e., supporting the feasibility of hot electron based solar cells. Recent femtosecond photoemission studies have, however, found extremely fast ( $<50 \mathrm{fs}$ ) electron relaxation at surfaces, in contrast to bulk dynamics $>1$ ps). The surface relaxation is highly sensitive to surface structure and provides an avenue to control electron thermalization for optimal energy conversion.

\section{Photoinduced Electron Transfer Processes in Homogeneous and Microheterogeneous Solutions \\ Whitten, D.G. \\ 716-275-1858 \\ $\$ 125,000$}

This work involves a study of light-induced electron transfer reactions in solution, the solid state, and thin films. It focuses on the potentially useful net chemical conversions that can occur as a consequence of single electron transfer quenching of excited states. Typically, these reactions are initiated by photoexcitation of visible or near ultraviolet light absorbing electron acceptors (or donors) and subsequent quenching by single electron transfer. In the cases examined in these studies, the quencher does not absorb light in those regions and typically contains a potentially fragmentable bond. The fragmentation, however, is only accessible in the radical ion due to a drastic and selective reduction of bond dissociation energies, which can occur for different species upon one electron oxidation or reduction. Donors that can be fragmentable from their radical cations include amines, diamines, aminoketones, aminoalcohols and pinacols. Acceptors that can undergo corresponding fragmentation include organic halides, ethers, and esters. Polymeric systems containing photoexcitable acceptors and fragmentable donors that can be reacted in both solid state and in solution have been designed and synthesized. Interestingly, fragmentation (and concurrent depolymerization) is observed even in the solid state where the lack of solvent and diffusional processes might be expected to significantly retard net photochemical electron transfer reactivity. Co-fragmentation reactions involving excited pinacols reacting with various organic halides have also been examined; when these reactions are carried out in the presence of oxygen, chain reactions for double fragmentation are observed, resulting in highly efficient liberation of acid and potentially interceptable radicals. 


\section{Rutgers, The State University of New Jersey Piscataway, NJ 08855}

\section{Department of Chemistry}

\section{Intramolecular Electron Transfer Across Synthetic Peptides: Effects of Structural Constraints, Secondary Structure, and Polarizable Side Chains \\ Isied, S.S. 908-445-3764

This project addresses the electron mediating properties of peptide bonds, amino acid side chains, and hydrogen bonds in the control of long range electron transfer in proteins. In this research, well defined, rigid peptide systems, where metal donors and metal acceptors are covalently attached at the peptide terminals, are assembled. Examples of these bridging peptide networks include oligoproline units, cyclic peptides, and peptides with $\alpha$-helical structures. Results from this work have clearly indicated that the connectivity of the donor to the acceptor (i.e., the number and type of covalent bonds connecting the donor and the acceptor) can play an important role in increasing the distance across which long-range electron transfer can be observed. For example, rapid electron transfer is observed across rigid helical peptides consisting of nine proline units, which is approximately equal to the diameter of a small protein. New experiments emphasizing the electronic interaction across hydrogen bonding networks and cross-linked cyclic peptides are currently being carried out.

\section{Stanford University Stanford, CA 94305}

\section{Department of Chemistry \\ 167. Energy Transfer Processes in Solar Energy Conversion \\ Fayer, M.D. \\ 415-723-4446}

$\$ 160,000$

Photoinduced electron transfer and electronic excitation transport are being studied in complex molecular systems experimentally and theoretically. Of particular interest are situations in which the molecular structure of the system or dynamics in the system play a significant role in the electron and excitation energy transport. Experimental methods include picosecond transient absorption and picosecond fluorescence. Recently the first theoretical description of electron transfer and geminate recombination for a donor and any number of acceptors distributed on the surface of a spherical micelle was obtained. This is a complex problem that required the determination of the radial distribution function of hard disks on the surface of a sphere to properly include excluded volume in the calculations. Both analytical theory and Monte Carlo simulations were used. Excitation energy transport in complex polymer systems has been studied. By properly processing polymeric solid samples it is possible to create nano-domains of chromophore tagged chains embedded in a photoinactive polymer host. The number of tagged chains in the nano-domains can be controlled. These clustered chromophore systems exhibit very rapid energy transport. Theory to describe excitation transport among systems of interacting polymer chains and interacting chromophore containing micelles was also developed and applied to experimental systems. In addition, experiments and theory on photoinduced electron transfer and geminate recombination in liquid and solid solutions were used to understand the role of molecular diffusion one electron transfer dynamics in liquids.

\section{University of Tennessee at Knoxville Knoxville, TN 37996}

\section{Department of Chemistry}

\section{Studies of Radiation-Produced Radicals and Radical Ions \\ Williams, T.F. \\ 615-974-3468

The objective of this project is to characterize both the structure and reactivity of selected organic free radical and radical ion intermediates generated by irradiation of molecular systems. Of particular interest is the study of the radical ions that are generated in the primary chemical processes resulting from the absorption of high-energy radiation, since these charged species play an important role in the mechanisms of both radiation and photochemical effects. Specific projects include structural and reactivity aspects of novel species including (a) 1,3- and 1,4-cycloalkanediyl radical cations where one electron is delocalized over two nonadjacent carbon centers, (b) twisted structures in olefin (1,2-diyl) radical cations, (c) bisallylic ( $5 \pi$ electrons) radical cations involving throughspace interactions, (d) distonic radical cations where spin and charge are separated in the same molecule, and (e) studies of thermal and photoinduced rearrangements in rigid matrices proceeding by hydrogen transfer, ring opening, ring closure, and sigmatropic shifts. There is a special focus on the photochemistry of radical cations in the visible region of the solar spectrum, with emphasis on the role of orbital, configuration, and state symmetry in going from the photoexcited state of the reactant to the ground state of the product.

\section{University of Texas at Arlington Arlington, TX 76019}

\section{Department of Chemistry and Biochemistry}

\section{Interfacial Chemistry at the Chalcogenide Semiconductor/Electrolyte Junction \\ Rajeshwar, $K$. 817-273-3810 \\ $\$ 98,859$}

This research involves two projects: (1) new electrosynthetic routes to the fabrication of chalcogenide semiconductors at support electrode surfaces; and (2) realtime insitu correlations of the interfacial chemistry and the electrical performance of a semiconductor electrode/ electrolyte junction. Proof-of-concept experiments have been completed and have opened a new route to the electrosynthesis of chalcogenide semiconductors that is based on a chemically-modified support electrode. Specifically, a sulfur-modified gold electrode is electroreduced in a 
medium containing a targeted metal jon (or ions) to yield immobilized metal sulfide particles at the gold surface. The utility of this approach for molecular-level ordering of the immobilized semiconductor particles and the role of the sulfur layer as a "template"is being investigated. A second topic is the development and use of new families of real-time/in situ probes for the characterization of semiconductor/electrolyte interfaces. These include the complementary probes, laser Raman spectroscopy and electrochemical quartz crystal microgravimetry. A photoelectrochemical version of the latter technique has been developed to facilitate microgravimetry measurements on metal/semiconductor interfaces under illumination. Experiments have been completed on the growth, corrosion, and stabilization dynamics of Fe-S, Cd-S, and Cd-Se based chalcogenide semiconductors.

\section{University of Texas at Austin Austin, TX 78712}

\section{Department of Chemistry and Biochemistry}

\section{Vectorial Electron Transfer in Spatially Ordered Arrays \\ Fox, M.A. 512-471-1811 \\ $\$ 180,000$}

Chemically modified semiconductor surfaces are being studied mechanistically as sites for controlled photomediated oxidation and reduction reactions. New methods for synthetic manipulation of the surfaces are being explored, along with new methods for preparation and characterization of coordination polymers and solar light harvesting polymeric layers. These polymeric coatings are then associated with optically transparent electrodes, producing a multicomponent system that can be used to probe quantitative aspects of electrocatalysis, electrosorption, and chemisorption. The use of new polymerization methods for preparing rectifying mono- and bilayer electrode coatings are being explored, with the attainment of vectorial migration of excitons and electrons being an ultimate project goal.

\section{Photoredox Processes at Polymer-Water Interfaces \\ Webber, S.E. 512-471-3633 \\ $\$ 105,000$}

This research effort concerns the use of chromophores covalently attached to amphiphilic polymers in order to enhance the yield of ionpairs produced in photoredox reactions. Using anionic polymerization methods, triblock polymers of polystyrene, vinyldiphenylanthracene (vDPA), and methacrylic acids have been prepared. The block length of the vDPA segment is relatively short, 3 to 4 units, while the other blocks are on the order of 200 units. These triblock polymers self-assemble into micelles. Excited-state electron transfer has been studied using a zwitterionic viologen (SPV) as a quencher. The fraction of singlet-state quenching events that lead to ionpair separation is relatively low (ca. 0.06) but the lifetime of the SPV anion radical is exceptional, with almost no decay over a $10 \mathrm{~ms}$. It was found that $\mathrm{C}_{60}$ or $\mathrm{C}_{70}$ and styrene would copolymerize with standard free-radical methods. The photophysical properties of the fullerene are strongly modified after copolymerization. The resulting polymers have the normal solution properties of polystyrene and one can easily prepare deeply colored solutions or films.

\section{Tulane University New Orleans, LA 70118}

\section{Department of Chemistry}

\section{Photoinduced Energy Transfer in Transition Metal Complex Oligomers Schmehl, R.H. 504-865-5573

Recent results have shown that multimetallic transition metal complexes can serve as effective sensitizers for photoelectrochemical cells based on wide band gap semiconductors. Photon to current efficiencies approaching unity have been achieved using a trimetallic $\mathrm{Ru}$ (II) diimine complex adsorbed on nanocrystalline $\mathrm{TiO}_{2}$ deposited on a $\mathrm{SnO}_{2}$ optically transparent electrode. Our efforts have focused on (1) understanding excitation energy migration in multimetallic transition metal complexes and (2) devising new light harvesting arrays capable of absorbing a higher fraction of incident photons per particle. Through systematic investigations of intramolecular energy transfer in bimetallic complexes, we have found bridging diimine ligands (linked bis-2,2'bipyridyl ligands) capable of mediating energy migration between adjacent metal centers with nearly $100 \%$ efficiency. In addition, simple synthetic strategies have been developed for preparing the most effective of these ligands: 1,4-bis-(2,2'-bipyrid-4-yl)benzene (bphb) and 1,4bis-(2,2', $6^{\prime}, 2^{\prime \prime}$-terpyrid-4'-yl)benzene (tpht). Using these ligands we have managed to show that multimetallic complexes can be prepared at interfaces via sequential reaction of complimentary components. For example, films having alternating $\mathrm{Ru}$ (II) and $\mathrm{Fe}$ (II) centers can be prepared by alternate reaction of aqueous $\mathrm{Fe}$ (II) and the complex $\left[R u(t p h t)_{2}\right]^{\text {2plus }}$ with a surface initially treated to have an attached terpyridine ligand. Dendrimeric surfaces can be prepared by alternate reaction of a $2,2^{\prime}-$ bipyridine modified surface with [Ru (II) (DMSO) $\left.{ }_{4} \mathrm{Cl}_{2}\right]$ and $\mathrm{bphb}$. These surfaces have light harvesting arrays of eight or more chromophores linked by a bridge capable of efficiently mediating energy migration to the reactive surface bound complex.

\section{Washington State University Pullman, WA 99164}

\section{Department of Chemistry}

\section{Investigations of Charge-Separation Processes in Metal Complexes Crosby, G.A. 509-335-5605}

This research is directed toward the elucidation of the excited states of metal complexes. Species containing $\mathrm{Rh}(\mathrm{III}), \mathrm{Pt}(\mathrm{II}), \operatorname{Re}(\mathrm{I})$, bimetal species $\left[\mathrm{M}_{1}, \mathrm{M}_{2}=\mathrm{Au}(\mathrm{I})\right.$, $\mathrm{Pt}(\mathrm{II}), \operatorname{Rh}(\mathrm{I}), \operatorname{Ir}(\mathrm{I})]$ and trimetal species containing $\operatorname{Rh}(\mathrm{I})$ and $\operatorname{Ir}(\mathrm{I})$ are under investigation. The intent is to synthesize stable complexes capable of utilizing incident 
radiation for chemical reactions, energy transfer, and storage. Analyses of luminescence decay times as a function of temperature, the measurement of relative polarizations of absorption and emission bands, and the determinations of the optical effects of intense magnetic fields (0-5 Tesla) are the principal tools of investigation. Recent data are providing values for the energy gaps separating states of disparate orbital parentages, the Arrhenius activation energies for photochemical reactions, and the paths of energy migration in excited systems. The ultimate goal of the research is to provide fundamental information on chargeseparation processes in well-defined metal complexes.

\section{Membrane-Organized Chemical Photoredox Systems \\ Hurst, J.K. \\ 509-335-7848}

$\$ 149,999$

This research is designed to improve our conceptual understanding of reaction mechanisms in two areas that are directly pertinent to solar photoconversion: (1) transmembrane charge separation across artificial bilayer membranes. Both areas are critical to developing membrane-based integrated photocatalytic systems for generation of fuels. The catalysis studies involve resonance Raman and electron paramagnetic resonance structural determination of reactive intermediates formed by ruthenium $\mu$-oxo ions. These analyses will be made in conjunction with steady-state kinetic measurements to identify the oxygen-evolving species. The transmembrane charge separation studies are directed at identifying the topographies and aggregation states of redox-active dopants in bilayer membranes that generate electron transport pathways and at developing multifunctional molecules that can act both as oxidative quenchers of photoexcited dyes and as transmembrane cotransporters of electrons and protons.

\section{University of Washington Seattle, WA 98185}

\section{Department of Biochemistry}

\section{Femtosecond Spectroscopy of Energy Transfer Dynamics in Photosynthetic Antennas \\ Nagarajan, $V$. \\ 206-543-1788

The goal of this project is to elucidate the pathways and mechanisms of electronic excitation transfer (EET) among the "antenna" complexes of photosynthetic bacteria, by the use of femtosecond pump-probe spectroscopy. The antennas are large arrays of pigment-protein complexes that collect light energy and transfer it efficiently to the "reaction center", where a series of electron-transfer steps is initiated, eventually culminating in the production of adenosine triphosphate. Each of the major antenna complexes has a distinctly different absorption spectrum. Transient absorption spectra acquired following excitation of specific antenna complexes are analyzed globally, with the goal of extracting a comprehensive picture of the EET process. The dynamics of intracomplex EET are probed by transient absorption anisotropy.

\section{Wayne State University Detroit, MI 48202}

\author{
Department of Chemistry
}

\section{Photoinduced Charge and Energy Transfer Processes in Molecular Aggregates \\ Endicott, J.F. 313-577-2607}

The major goals of this research project involve the systematic investigation of models that describe various aspects of the photoinduced transfer of charge, or the migration of energy between donor and acceptor transition metal-complexes. Research involves the design and synthesis of molecular systems to be used as mechanistic probes and the characterization of photochemical transients using very sensitive detection techniques. Work in progress varies from studies of the general problem of electronic coupling in donor-acceptor systems to specific problems relating to the pathways for intramolecular energy transfer from the lowest energy excited state of chromium(III). Considerable electronic coupling of donor and acceptor seems to be an important characteristic of polynuclear transition-metal complexes with $\mathrm{CN}^{-}$bridging groups, and the effects of this coupling are manifested in excited-state electron-transfer rates, ground-state spectroscopic behavior, and electrochemical behavior. In complexes for which the donor excited state is $\left({ }^{3} \mathrm{CT}\right) \mathrm{Ru}$ (bpy) ${ }_{2+3}$ and the acceptor is a covalently linked metal complex, the donor and acceptor centers usually behave reasonably independently. The systematic comparison of the properties of some homologous series of complexes has indicated that the donor-acceptor coupling inferred from electrochemical measurements is consistently much larger than that inferred from a Hush-type of interpretation of the spectroscopic measurements. These and some other observations on this class of complexes are accommodated by a simple vibronic model in which the electronic and nuclear motions are coupled. Studies in progress are designed to examine some implications of this model.

\section{Wichita State University Wichita, KS 67260}

\section{Department of Chemistry}

\section{Mixed-Metal, Multielectron Photocatalysts for Solar Energy Conversion \\ Rillema, D.P. $\$ 130,000$ 316-689-3120 \\ (16 months)}

The design, synthesis, and examination of the photophysical properties of potential solar energy photocatalysts is the focus of this research. The low-temperature luminescence properties of $\left[\operatorname{Re}\left(4,7-\mathrm{Me}_{2} \text { phen }\right)(\mathrm{CO})_{3} \mathrm{py}\right]^{+} \quad\left(4,7-\mathrm{Me}_{2}\right.$ phen=4,7-dimethyl1,10 phenanthroline, py=pyridine) as a crystal or dissolved in an ethanol-methanol glass were investigated using laser line narrowing techniques. Single crystal absorption and luminescence spectra at temperatures below $6 \mathrm{~K}$ showed that the emitting state in the crystalline environment was triplet-ligand centered ( $\left.{ }^{3} \mathrm{LC}\right)$. In an 
ethanol-methanol glass, the luminescence spectra indicated that a largely ${ }^{3} \mathrm{LC}$ assignment was also in this matrix; however, lifetime measurements provided some evidence for a contribution from the nearby triplet metalto-ligand charge transfer $\left({ }^{3} \mathrm{MLCT}\right)$ state. Measurements of the Zeeman effect were made confirming the ${ }^{3} \mathrm{LC}$ assignment in both environments. The results of temperature dependent behavior in solution, however, placed the emission activity within the ${ }^{3}$ MLCT emitting manifold, where the series of $\operatorname{Re}(\mathrm{L}-\mathrm{L})(\mathrm{CO})_{3} \mathrm{py}^{+}, \mathrm{L}-\mathrm{L}=3,4,7,8-\mathrm{Me}_{4}$ phen, 4,7$\mathrm{Me}_{2}$ phen, 2,9-Me $\mathrm{Me}_{2}-4,7-\mathrm{Ph}_{2}$ phen, 5,6-Me 2 phen, 5-Phphen, $2,9-\mathrm{Me}_{2}$ phen and phen, were examined. The emission lifetimes of $\operatorname{Re}\left(3,4,7,8-\mathrm{Me}_{4}\right.$ phen $)(\mathrm{CO})_{3} \mathrm{py}^{+}$actually increased with temperature, reached a maximum, and then decreased in the usual manner. The cause for this unusual behavior is currently under investigation but may be related to deactivation from a thermally accessible state lying above the lowest energy excited state or due to phonon relaxation phenomena.

\section{Chemical Physics}

\section{Aerospace Corporation Los Angeles, CA 90009}

\section{Space and Environment Technology Center}

\section{A Shock Tube Study of the Reactions of Hydroxyl Radicals with Combustion Species \\ Koffend, J.B.; Cohen, $N$. 310-336-7412

The project objective is the measurement of rate coefficients for reactions of $\mathrm{OH}$ radicals necessary for understanding and predicting oxidation and combustion kinetics of hydrocarbon fuels and anthropogenic industrial pollutants. The experiments are being performed in a shock tube under combustion conditions (near $1200 \mathrm{~K}$ and 1 atmosphere pressure). Under such conditions, reactions with $\mathrm{OH}$ radicals are often the most important step in the decomposition process. Transition-state theory (TST) calculations are also being carried out to relate the measurements to other data at lower temperatures so that reliable temperature dependences of the rate coefficients under study can be established. The calculations also assist in the formulation of semiempirical rules for predicting the rate coefficients of species for which no data are available. Experimental measurements were completed for a series of hydrocarbons with a newly renovated facility. Compounds measured include ethane, hexane, heptane, octane, nonane, decane, 2-methyl pentane, 3methyl pentane, and 2,2,3-trimethyl pentane. Results are in very good agreement with transition-state theory calculations.

\section{University of Akron Akron, OH 44325}

\section{Department of Chemistry}

\section{Generalizations Concerning Vibrational and Rotational Energy Redistribution within Polyatomic Molecules \\ Perry, D.S. \\ 216-375-6825 \\ $\$ 81,982$}

This project employs high-resolution infrared doubleresonance spectroscopy to explore the possibility of establishing broadly based generalizations about the rate and mechanism of intramolecular vibrational energy redistribution (IVR). The presence of rapid IVR determines the collisional and reactive properties of vibrationally excited molecules in combustion systems or wherever they occur. The role of molecular flexibility in accelerating IVR, the dependence on the nature of the initially prepared vibration, and a possible correlation between rate and mechanism are explored in this work. Specific molecular systems include propyne, methanol, and methylamine, which will be studied in the 3000 to $7000 \mathrm{~cm}^{-1}$ energy range. The experiments resolve clumps of discrete molecular features, called molecular eigenstates, for which the good quantum numbers are completely assigned. The needed information about the rate and mechanism of IVR is contained in the frequencies and intensities of these discrete features. The experimental work is supported by random matrix calculations, which are capable of quantifying the contributions of anharmonic and Coriolis (3 types) coupling mechanisms.

\section{Arizona State University Tempe, AZ 85287}

\section{Department of Chemistry}

\section{Electronic Structure and Reactivities of Transition Metal Clusters \\ Balasubramanian, $K$. 602-965-3054

The objective of this research is to seek answers to fundamental and intriguing questions pertinent to the electronic structure and reactivities of transition metal clusters. The geometries, binding energies, energy separations of excited states, ionization potentials, and other properties of clusters, including their reactivities, are theoretically computed as functions of cluster size. Theoretical studies on the dimers and trimers are focused on the energy separations $\left(T_{e}\right)$ of several excited states and their spectroscopic constants $\left(\mathrm{r}_{\mathrm{e}}, \omega_{\mathrm{e}}, \mu_{\mathrm{e}}\right.$. Computations on the potential energy surfaces are undertaken to shed light on the reactivity of these species. Spectroscopic constants of several low-lying electronic states of PtN, WN, and other transition metal nitrides, as well as carbides are computed including spin-orbit effects. The observed spectra are too complex to explain without theoretical studies. Comparisons with observed spectra are made. Systematic studies of $\mathrm{Pt}_{3} \mathrm{Au}_{2}$ found in inorganic complexes as well as $\mathrm{Pt}_{3} \mathrm{Ag}_{2}$ are being made. Geometries and energy separations of transition metal clusters such as $\mathrm{Pt}_{4}, \mathrm{Pd}_{4}, \mathrm{Ir}_{3}, \mathrm{Zr}_{3}$, 
etc. have been recently computed using high-level relativistic ab initio methods. The potential energy surfaces of transition metal dimers with ethylene and butadiene will be computed to gain insight into the nature of organometallic ring closure reactions. Specific reactions being studied are $\mathrm{W}_{2}+\mathrm{Cu}_{2}, \mathrm{Ag}_{2}$ and $\mathrm{Au}_{2}$ with ethylene as well as $\mathrm{CO}$ is being investigated through the computation of the potential energy surfaces. Metal complexes with benzene such as $\mathrm{Pt} \pm$ benzene, $\mathrm{Pt}$-benzene, etc. are being investigated. The interaction of $\mathrm{Pt}_{\mathrm{n}}, \mathrm{Pd}_{\mathrm{n}}, \mathrm{Rh}_{\mathbf{n}}$ and $\mathrm{Ir}_{\mathrm{n}}$ clusters with $\mathrm{CO}$ is being studied. These studies use complete active space MCSCF (CASSCF) followed by multireference configuration interaction (MRCI) computations that include several million configurations. Spin-orbit effects are included using the relativistic configuration interaction (RCI) method.

\section{Generation Detection and Characterization of Gas-Phase Transition-Metal Aggregates and Compounds Steimle, T.C.} 602-965-3265

The generation and characterization of new transition metal compounds, in their gaseous phase, are being performed. A well-collimated supersonic molecular beam sample of diatomic and polyatomic iridium and platinum compounds is generated by skimming the output of a laser ablation/reaction source. Near natural linewidth optical spectra (typical resolution $50 \mathrm{MHz}$ FWHM) are recorded using single mode cw-dye laser-induced fluorescence spectroscopy. The observed Stark shifts and magnetic hyperfine structure are interpreted in terms of plausible bonding models. A systematic study of the ionic character of PtC, PtN, PtO, and PtS, as determined from the measured permanent electric dipole moments, has been completed. Periodic trends in bonding were established. The dipole moments of these Pt compounds are unexpectedly small (typically 1.5. D) compared to other known transition metal oxides, nitrides, carbides, and sulfides (typically $4.0 \mathrm{D}$ ). A similar study on Ir compounds is in progress. Numerous spectral features observed in the reaction of $\mathrm{Pt}$ and $\mathrm{Ir}$ with $\mathrm{CH}_{4}$ and $\mathrm{NH}_{3}$ are tentatively assigned as $\mathrm{Pt}$ and $\mathrm{Ir}$ atoms bound to polyatomic ligands. Pump/probe microwave optical double resonance spectroscopy of metal compounds is being performed to determine the structural properties of the ground electronic state.

\section{University of Arizona Tucson, AZ 85721}

\section{Department of Chemistry}

\section{Chemical Activation of Molecules by Metals: Experimental Studies of Electron Distributions and Bonding \\ Lichtenberger, D.L. 602-621-4749 \\ $\$ 105,000$}

The continued purpose of this research program is to obtain detailed experimental information on the different fundamental ways metals bond and activate organic molecules. Our approach is to directly probe the electronic interactions between metals and molecules through a wide variety of ionization spectroscopies and other techniques, and to investigate the relationships with bonding modes, structures, and chemical behavior. During this period of the project we have (1) accomplished the highresolution gas phase photoelectron spectra of $\mathrm{C}_{60}$ and $\mathrm{C}_{70}$, (2) characterized the electronic features of imaging $\mathrm{C}_{60}$ on gold by STM, (3) evaluated the orbital distributions of $\mathrm{C}_{60}$ and the bonding interactions with metals, (4) revealed details of the bonding of phosphines to metals, including the subtleties of a geometrical twist in sterically crowded situations, (5) determined the formal electron distribution in the bonding of $\eta^{3}$-cyclopropenyl with metals, (6) related gas-phase ionization energies to electron transfer kinetics and ion solvation thermochemistry of metallocenes, (7) correlated lone-pair ionization energies with proton affinities for a variety of amino acids and related compounds, (8) examined sigma-pi interactions in non-conjugated polyalkynes, (9) characterized extensive metal-ligand $\pi$ interactions in metal-acetylide compounds, and (10) continued to develop the experimental and theoretical methods for these studies.

\section{University of California, Los Angeles Los Angeles, CA 90024}

\section{Department of Chemistry and Biochemistry}

\section{High-Resolution Raman Spectroscopy of Complexes and Clusters in Molecular Beams \\ Felker, P.M. \\ 310-206-6924 \\ $\$ 98,000$}

The project objectives are twofold. The first is to develop methods of nonlinear Raman spectroscopy for application in studies of sparse samples. The second is to apply such methods to structural and dynamical studies of species (molecules, complexes, and clusters) in ultracold supersonic molecular-beam samples. In the past year we have extended the application of mass-selective ionizationdetected stimulated Raman spectroscopies (IDSRS) to the study of intermolecular vibrational transitions in molecular clusters. The study of such vibrations is central to an understanding of intermolecular forces. In studies of naphthol-water and -ammonia complexes, we have shown that certain types of hydrogen-bond vibrations are prominently Raman active. Further, we have extended our study' of intermolecular vibrations in aromatic ${ }_{n}$ clusters to the photoreactive trimers and tetramers of naphthalene. We have also come up with a picture as to how intermolecular vibrations obtain significant Raman activity. The hypothesis is that such activity can be ascribed to those intermolecular vibrations that involve the libration of monomer moieties having large permanent polarizability anisotropies. This hypothesis explains virtually all of the IDSRS observations that we have made thus far pertaining to intermolecular transitions and represents a powerful predictive tool 'for planning future experiments. During the course of the year ahead, with an upgrade of our current apparatus, we plan to obtain Raman spectra at a resolution that is an order of magnitude greater than is possible now and to study the vibrational spectroscopy of new classes of jet-cooled species that are relevant in combustion processes (i.e., radicals and ions). 


\section{University of California, Santa Barbara \\ Santa Barbara, CA 93106}

\section{Department of Chemistry}

\section{Interactions of Highly Vibrationally Excited Molecules with Clean Metal Surfaces \\ Wodtke, A.M.; Auerbach, D.J. 805-893-8085

This project will involve a series of exploratory experiments to study the interaction of highly vibrationally excited molecules with single-crystal metal surfaces. Stimulated emission pumping will be used to prepare single quantum states of vibrationally excited molecules in a beam incident upon a single-crystal surface within an ultrahigh vacuum surface-science chamber. This technique will allow study of the interactions of molecules in a single quantum state and with up to several hundred joules per mol of vibrational energy. Auger electron spectroscopy, temperature programmed desorption, X-ray photoelectron spectroscopy, and time-resolved mass spectroscopy will be used to map out the relative reaction probability as a function of the reactant vibrational quantum numbers. The studies will be perhaps the first surface reactive studies done with beams prepared in individual quantum states and the first studies of molecules in highly excited. vibrational states. They promise to advance knowledge of how reactant vibrational energy influences (1) activated dissociative adsorption, (2) nondissociative or molecular adsorption, and (3) direct ("Eley-Rideal") reactions between incident and absorbed species. The proposed studies require a combination of techniques from the fields of surface science, molecular beams, and laser spectroscopy. The experiment will be a collaborative effort between the University of California-Santa Barbara (Wodtke) and IBM Almaden Research Center (Auerbach).

\section{Catholic University of America Washington, DC 20064}

\section{Department of Chemistry}

\section{Studies of Combustion Kinetics and Mechanisms \\ Slagle, $I$. $\$ 120,000$ 202-319-5383}

The goal of this research is to obtain new quantitative knowledge of the kinetics and mechanisms of the elementary reactions of polyatomic free radicals, which are important in hydrocarbon combustion processes. Polyatomic free radicals are generated in a heatable (up to $1100 \mathrm{~K}$ ) flow reactor by the photodecomposition of suitablemolecules using a pulsed uv-laser. The ensuing reactionsare monitored in time-resolved experiments using photoionization mass spectrometry. In order to obtain basic information regarding the reactivity of these free radicals, reaction rate constants are measured as a function of temperature and pressure (0.5 to 25 torr) and, when possible, the primary reaction products are determined and their branching fractions measured. These experimental studies are coupled, where possible, with theoretical ones to obtain an improved understanding of the factors governing reactivity and to provide a rational basis for extrapolating the observed kinetic behavior of free-radicali processes from laboratory conditions to the harsher environment of actual combustion processes. Recent studies. have focused on the unimolecular decomposition of free radicals $\left(\mathrm{n}+\mathrm{C}_{7} \mathrm{H}_{9}\right)$ and $\mathrm{C}_{2} \mathrm{H}_{3}$ and the reactions of vinyl radical $\left(\mathrm{C}_{2} \mathrm{H}_{3}+\mathrm{O}_{2}\right.$ and $\left(\mathrm{C}_{2} \mathrm{H}_{3}+\mathrm{C}_{2} \mathrm{H}_{2}\right)$.

\section{University of Chicago Chicago, II 60637}

\section{James, Franck Institute}

\section{Chemical Reaction Dynamics: of Combustion Intermediates and Products. \\ Butler, L.J. 312-702-7206 \\ $\$ 145,000$}

The series of experiments pursued for this project develop a unified understanding of bond-selective molecular dissociation processes important in combustion: To investigate how local electronic excitation of a polyatomic molecule can result in selective decomposition channels over other energetically allowed ones, this work utilizes photofragment velocity and angular distribution analysis in a crossed laser-molecular beam apparatus. Polarized emission spectroscopy provides complementary information by probing the early dissociation dynamics. Work this year included emission spectroscopy to probe the dissociation dynamics of $\mathrm{H}_{2} \mathrm{~S}$ from $\mathrm{a}$ conical intersection in the Franck-Condon region to the $\mathrm{H}+\mathrm{SH}$ product exit channel; a collaborative theoretical project that compares the adiabatic prediction for the branching ratio between C-S and $\mathrm{S}-\mathrm{H}$ bond fission in $\mathrm{CH}_{3} \mathrm{SH}$ excited in the first ultraviolet absorption band with experimental results; an experiment that demonstrates how to calibrate the absolute photoproduct branching ratio using mass spectrometric detection when the ion fragmentation patterns of the radical products are unknown (the C-C:C-C1 branching ratio in chloroacetone photolysis by calibrating the signal intensities at $\mathrm{Cl}+$ and $\mathrm{CH}_{2} \mathrm{CO}+$ using acetyl chloride photodissociation); and a.study of the competition between $\mathrm{H}_{2}$ elimination and $\mathrm{C}-\mathrm{N}, \mathrm{C}-\mathrm{H}$, and $\mathrm{N}-\mathrm{H}$ bond fission in methyl amine at $222 \mathrm{~nm}$ and of C-Br and C-I bond fission in 1,3-bromoiodopropane:

\section{Quantum Dynamics of Fast Chemical Reactions \\ Light, J.C. 312-702-7197 \\ $\$ 102,000$}

In this research we want to determine accurate values of thermal rate constants and state-to-state cross sections for elementary bimolecular reactions in the gas phase including photodissociation. In these studies we develop and use accurate and efficient quantum methods. The thermal rate constants have been calculated both from the quantum thermal averaged flux-flux correlation function (evaluated by diagonalizing the Hamiltonian in a threebody DVR) and from the cumulative reaction probability, N(E): Recently we have developed methods that use only real square integrable representations on a finite (small) range-in order to represent the continuum scattering processes. This is highly. advantageous in that no artificial imaginary. boundary potentials are required, and real: 
arithmetic can be used almost throughout. We have applied these to the photodissociation of van der Waals molecules and the UV photodissociation of a model for methyl mercaptan. Applications to other 3-D reactions are in progress. We have also treated the dynamics of electronically nonadiabatic collisions using the two diabatic surfaces, together with an appropriate interaction term. Nonadiabatic effects are common in photodissociation and in some chemical exchange reactions. An additional focus has been on the development of a "quantum transition state theory" based on operator expressions for $N(E)$ with semiclassical log derivative boundary conditions or on the finite range methods mentioned above.

\section{University of Colorado Boulder, CO 80309}

\section{Department of Chemistry and Biochemistry}

\section{Laser Photoelectron Spectroscopy of Ions Ellison, G.B. 303-492-8603

This enterprise uses photoelectron spectroscopy to study the properties of negative ions and radicals. The essence of the experiment is to cross a $0.6 \mathrm{keV}$ mass-selected ion beam $\left(\mathrm{M}^{-}\right)$with the output of a $\mathrm{CW}$ laser, $\hbar \omega_{0}$. The resultant detached photoelectrons with kinetic energy, KE, are energy analyzed by an electrostatic hemispherical analyzer: $\mathrm{M}^{-}+\hbar \omega_{0} \rightarrow \mathrm{M}+\mathrm{e}(\mathrm{KE})^{-}$. Analysis of the photoelectron spectra provides molecular electron affinities, vibrational frequencies, and electronic splittings of the final radical, M, as well as the relative molecular geometries of the radical and its parent anion. In combination with other experimental techniques, a wide range of thermochemical properties of the benzyl, phenyl and allyl radicals have been determined. For example: using a flowing afterglow/selected ion flow tube instrument, the rates of reaction of the amide ion with benzene and inverse reaction of ammonia with the phenide ion were determined. From these rates, the free energy change of the reaction was determined. Combining this with the gas phase acidity of ammonia gave the gas phase acidity of benzene. Combining this with computed values of the enthalpy of deprotonation of benzene and the measured electron affinity for the phenyl radical, the hydrogen bond energy in benzene as well as the heat of formation of the phenyl radical were determined.

\section{Time-Resolved FTIR Emission Studies of Laser Photofragmentation and Chain Reactions \\ Leone, S.R. 303-492-5128

Current research involves two major thrusts related to combustion processes. One is to develop methods for studying radical-radical reactions, and the other is to study single-collision energy transfer and reactive dynamics. Both methods utilize time-resolved Fourier transform infrared emission, which combines a commercial FTIR spectrometer with a high repetition rate excimer laser. Using high energy laser pulses and a fast-flow reactor, a new series of radical-radical processes are being studied: $\mathrm{C}_{2} \mathrm{H}_{5}+\mathrm{O}$ and $\mathrm{C}_{2} \mathrm{H}+\mathrm{O}$. The $\mathrm{OH}(\mathrm{v})$ products of these reactions and the $\mathrm{CH}+\mathrm{O}$ reaction are characterized. In addition, an unexpected HOI vibrationally excited product has been observed for the reaction of $\mathrm{O}$ atoms with $\mathrm{C}_{2} \mathrm{H}_{5} \mathrm{I}$ and other alkyl iodides, suggesting a five-center reaction facilitated by a long-lived collision complex. A vibrationally and rotationally resolved study of collisions of fast $\mathrm{H}(\mathrm{D})$ atoms with $\mathrm{HF}$, which produce both inelastic T-V excitation transfer as well as the $\mathrm{H}$ atom exchange reaction, has also been completed. Detailed comparisons of the experimental and theoretical results for these systems provide one of the most exacting tests of vibrational and rotational dynamics in a system with competing pathways.

\section{Columbia University New York, NY 10027}

\section{Department of Chemistry}

\section{Energy Partitioning in Elementary Gas-Phase Reactions Bersohn, $R$. 212-854-2192}

Previous studies on the $\mathrm{CO}$ and $\mathrm{H}$ atom products of the $O\left({ }^{3} \mathrm{P}\right)+$ acetylene reaction have been extended to the reactions of $\mathrm{O}\left({ }^{3} \mathrm{P}\right)$ with a series of alkynes. The most startling discovery is that the $\mathrm{CO}$ products are invariably vibrationally and rotationally cold in spite of the fact that the reactions are very exothermic. The general explanation is as follows. The $O$ atom attaches itself to an unsaturated carbon atom forming a vibrationally excited $\mathrm{C}-\mathrm{O}$ bond. However, a hydrogen atom or alkyl radical is bound to that same carbon atom; the $\mathrm{CO}$ molecule cannot be liberated until the $\mathrm{H}$ atom or alkyl radical migrates to an adjacent carbon atom. The energy within the $\mathrm{CO}$ bond is used to overcome the potential barrier to the 1,2 migration. The substituted ketene is then formed decomposes too rapidly for vibrational energy to flow back to the $\mathrm{CO}$. On the other hand, the linearity of the ketene CCO group produces a rotationally cold $\mathrm{CO}$ molecule. The larger alkynes yield a variety of radicals whose identity and ionization potentials will be determined by vacuum ultraviolet photoionization. Experiments similar to the above are planned for the reactions of $O\left({ }^{3} \mathrm{P}\right)$ with alkenes

\section{Laser-Enhanced Chemical Reaction Studies \\ Flynn, G.W. 212-854-4162}

$\$ 130,000$

This project employs extremely high resolution infrared diode lasers to study fundamental combustion and collision dynamics and photochemical reaction processes. High energy atoms, molecules, and chemically reactive radicals, produced by excimer laser photolysis or dye laser excitation, are used as reagents to investigate collisional excitation, collisional quenching, and chemical production of individual rotational and vibrational states of molecules. Translational energy recoil of the target molecules is determined by measuring the time-dependent Doppler profile of the molecular infrared transitions. This experimental method has been used to probe the quenching step in the famous Lindemann unimolecular reaction model in which vibrationally hot molecules with chemically significant amounts of energy are cooled by collisions with a cold bath molecule. Quantum state and velocity 
distributions have been determined for carbon dioxide molecules recoiling from pyrazine, hexafluorobenzene, and pyridine molecules containing typically $5 \mathrm{eV}$ of vibrational energy. Experiments of this type have provided the first glimpses of the separate behavior of the translational and rotational degrees of freedom of the recoiling bath molecules during the relaxation of highly vibrationally excited donors. The presence of "super collisions" in the quenching of high energy pyrazine molecules has been verified, but similar, remarkably high energy quenching processes seem to be less significant in vibrational energy loss from hexafluorobenzene. In these rare "super collision" events, the majority of the lost vibrational energy is carried away by the translational motion of the colliding partners. The detailed chemical and collision dynamics of a number of processes are being studied using infrared diode lasers to probe the reaction products and their energy distributions and using optical parametric oscillators to prepare single initial quantum states of the reactants. The data obtained in all of these experiments is of fundamental as well as practical interest in testing theoretical computations based on approximate potential energy surfaces, in assessing the role of statistics in the determination of quantum state product distributions, and in providing an improved understanding of combustion and atmospheric chemical processes.

\section{Single-Collision Studies of Hot Atom Energy Transfer and Chemical Reaction Valentini, J.J. \\ $\$ 92,000$}

This research project addresses the dynamics of chemical reactions that are important in combustion processes, or that serve as prototypes of important combustion reactions. The reactions being studied now involve free radical species, such as $\mathrm{H}, \mathrm{OH}$, and $\mathrm{CH}_{3}$, reacting with hydrocarbons, such as $\mathrm{CH}_{4}$. The major issue of interest is whether the dynamics of atom + polyatom reactions, which involve the concerted motion of many atoms in the polyatom reactant, are like those of the well understood and much simpler atom + diatom reactions. Both experimental and computational studies are employed to answer this question. The experiments use laser pulses to create the free radical reactant via photolysis, while other laser pulses characterize the energy distributions in the products, under single-collision conditions. The computational simulations are quasi-classical trajectory calculations. State-to-state reactive cross sections are revealed by the experiments and used to provide a rigorous test of the computational simulations of the reactions. When validated by comparison with experimental results, the computational simulations provide insight about the dynamics of combustion reactions, and will help develop models of these reactions that have significant predictive power.

\section{Cornell University Ithaca, NY 14853}

\section{Department of Applied and Engineering Physics}

\section{Spectroscopy of Combustion Radicals Cool, T.A. 607-255-4191 $\$ 70,000$}

A basic research need in support of the thermal processing of DOE hazardous chemical wastes is studies of the combustion of halogenated organic compounds with emphasis on reaction pathways leading to the formation of chlorinated aromatic compounds. Two studies using laser based detection of radicals and major species of importance in chlorocarbon combustion are being pursued. One of these studies, directed toward the improvement of current chemical kinetic models, employed three different laser-ionization techniques for species profile measurements in chlorocarbon seeded methane/oxygen flames. A significant finding is the observation that the chlorine atom concentration is suppressed throughout the primary flame zone associated with the production of $\mathrm{H}, \mathrm{O}$, and $\mathrm{OH}$ radicals, but increases rapidly, by the reconversion of $\mathrm{HCl}$ to $\mathrm{Cl}$, within an extended flame zone. The production of $\mathrm{Cl}$ in the extended flame zone may play a role in the formation of traces of complex chlorocarbons in the postflame environment. A second study of fluorescence quantum beats in cyanogen is underway, which has led to: (1) an original theoretical description of previously unreported magnetic modulations of quantum beats, and (2) the first demonstration of-the use of quantum beat spectroscopy for the study of the collisional depolarization of molecular fluorescence:

\section{Laboratory of Atomic and Solid State Physics}

\section{Photochemical Dynamics of Surface-Oriented Molecules Ho, W. 607-255-3555}

$\$ 110,000$

(15 months)
The main objective of the project is to understand the dynamics of surface photochemistry. The experimental approach lies in measuring the translational distribution of molecules photochemically desorbed by nanosecond and femtosecond laser pulses. The microscopic dynamics of desportion are revealed by the nonlinear dependence of the photoyield on the laser fluence, the dramatic variation of the translational energy on the laser fluence, and the time response of the photoyield and translational energy as a function of the time delay between a pair of laser pulses. Theoretical modeling shows that femtosecond laser pulses lead to a high degree of substrate electronic excitation. The hot electrons undergo multiple resonant electronic scatterings from the adsorbed molecules and excite repeatedly the vibrational states of the adsorbed molecules. The dynamics of such vibrational heating, which is especially efficient for femtosecond laser pulses, explicitly determine the bond-dissociation process. Experiments have been carried out on a model system of molecular oxygen desorbed from Pt (111) surface by 80 femtosecond laser pulses. The multiple electronic excitation mechanism is unique to photochemistry with femtosecond laser pulses and is not observed with nanosecond laser pulses. 


\section{Department of Chemistry}

\section{Studies of Combustion Reactions at the State-Resolved Differential Cross Section Level \\ Houston, P.L. 607-255-4303 \\ $\$ 102,664$}

The technique of product imaging is being used to investigate several processes important to a fundamental understanding of combustion. The imaging technique produces a "snapshot" of the three-dimensional velocity distribution of a state-selected reaction product. Research in three main areas is planned. First, differential cross sections are being measured for several reactions, perhaps the most important of which is the $\mathrm{H}+\mathrm{O}_{2}$ reaction. Second, the imaging technique will be used to detect only zero kinetic energy fragments from a photodissociation near threshold. Since these fragments are produced primarily when the photolysis light is resonant with an internal level of the activated complex, it should then be possible by scanning the photolysis light to obtain a "spectrum" of the transition state. Third, the imaging technique is being used to learn the distribution of translational energy when a highly vibrationally excited molecule collides under well-defined single-collision conditions with a partner. This distribution of translational energy is directly related to the distribution of vibrational energy removed by collisional deactivation, a quantity of importance to a theoretical understanding of two important combustion processes, unimolecular dissociation and radical recombination.

\section{Nuclear Science and Engineering Program}

\section{Interactions of Highly Charged Ions with Atoms at keV Energies \\ Kostroun, V.O. $607-255-4991$ \\ $\$ 300,000$}

This is an experimental project to investigate interactions between very highly charged ions and atoms at kinetic energies characteristic of thermonuclear fusion and astrophysical plasmas. The fundamental processes studied include single, double, and multiple-electron capture by the projectile, target ionization, and de-excitation of the highly excited projectile states formed in collisions. The data obtained are useful for modeling plasma behavior and contribute to a general understanding of atomic collisions. Highly charged ions are produced by the Cornell superconducting solenoid, cryogenic electron beam ion source, CEBIS. Extracted ions are charge-selected and decelerated to energies around $100 \mathrm{qeV}$ (where $\mathrm{q}$ is the ion charge). The $q+$ ions interact with a gas target, and angular distributions of scattered $(q-1)+$ projectiles associated with definite energy gains are measured at different scattering angles. The observed angular distributions of $\mathrm{Ar}^{\left(\mathrm{q}^{-1)+}\right.}$ projectiles in $\mathrm{Ar}^{\mathrm{q}+}(8 \leq \mathrm{q} \leq 14)$ on Ar collisions at 70$100 \mathrm{qeV}$ are not completely understood at present in terms of the molecular Coulomb barrier model. Total energy gain spectra integrated over all angles reflect the density of projectile capture states. The spectra for odd projectile charge states differ from those for even states, and efforts are underway to understand this difference.

\section{Emory University Atlanta, GA 30322}

\author{
Department of Chemistry
}

\section{Theoretical Studies of Combustion Dynamics \\ Bowman, J.M. \\ 404-727-6592}

$\$ 93,540$

The objective of this research project is to carry out detailed calculations of dynamical processes of importance in gas-phase combustion. The emphasis is on bimolecular chemical reactions of polyatomic molecules, and unimolecular reactions of importance in the formation and destruction of key radicals. In addition to their importance in combustion, the systems chosen for study are also being studied experimentally. Several new research projects have been completed or initiated in the past year. These include quantum calculations of the $\mathrm{H}+\mathrm{C}_{2} \mathrm{H}_{2} \rightleftharpoons \mathrm{H}_{2}+\mathrm{CCH}$ reaction, with a focus on the effect of vibrational excitation of $\mathrm{C}_{2} \mathrm{H}_{2}$ on the reaction cross section. Another nearly completed project is the calculation of positions and widths of more than 100 resonances of $\mathrm{HCO}$ and DCO. As part of this project, the B-X fluorescence spectra of the bound and resonance states of $\mathrm{HCO}$ and DCO are being calculated using an ab initio force field for the B-state and coordinate-dependent transition moment. The absorption spectrum of $\mathrm{HO}_{2}$ has been calculated using a slightly adjusted potential, and semiempirical dipole moment surface. Finally, two new projects on energy transfer in $\mathrm{Ar}-\mathrm{HCO}$ and $\mathrm{Ar}+\mathrm{HO}_{2}$ have begun. These are quantum coupled-channel scattering calculations of state-to-state energy transfer from and to highly excited vibrational states.

\section{Kinetics of Elementary Processes Relevvant to Incipient Soot Formation \\ Lin, M.C. 404-727-2825 \\ $\$ 101,262$}

The kinetics and mechanisms of phenyl radical reactions with a number of molecular reactants relevant to hydrocarbon combustion processes have been successfully investigated for the first time with the cavity ring-down (CRD) technique. The absolute rate constants determined by the CRD technique at room temperature for the $\mathrm{C}_{6} \mathrm{H}_{5}$ reactions (with $\mathrm{NO}, \mathrm{O}_{2}, \mathrm{HBr} / \mathrm{DBr}, \mathrm{CH}_{2} \mathrm{O}, c-\mathrm{C}_{5} \mathrm{H}_{10}$, c$\mathrm{C}_{6} \mathrm{H}_{12}, \mathrm{C}_{2} \mathrm{H}_{2}, \mathrm{C}_{2} \mathrm{H}_{4}$, etc.) correlate well with those for analogous $\mathrm{CH}_{3}$ reactions. For the reactions with $\mathrm{C}_{2} \mathrm{H}_{2}$ and $\mathrm{C}_{2} \mathrm{H}_{4}$, we have also analyzed the results obtained as functions of temperature and pressure with the RRKM theory based on ab initio molecular orbital (MO) and thermokinetic data. Additionally, MO calculations using the spin-projected UMP3 method have been carried out for the reaction of $\mathrm{C}_{6} \mathrm{H}_{5}$ with $\mathrm{O}_{2}$, for which more than a dozen stable reaction intermediates have been identified. The role of these intermediates, i.e., the isomers of $\mathrm{C}_{6} \mathrm{H}_{5} \mathrm{OO}$, in the oxidation of the phenyl radical at high temperatures is under investigation. 


\section{Florida State University Tallahassee, FL 32306}

\section{Supercomputer Computations Research Institute}

\section{Ab Initio Electronic Structure Studies of Formation and Reactions of Polycyclic Aromatic Hydrocarbons in Flames \\ Cioslowski, J.; Moncrieff, $D . \quad \$ \mathbf{\$ 1 2 5 , 0 0 0}$ 904-644-4885}

Because of their ubiquity, polycyclic aromatic hydrocarbons (PAHs) are of major interest to diverse branches of chemistry. Their formation in various pyrolytic processes, including combustion of fuel, had profound consequences to environmental protection, as many of the PAHs are well known as potent carcinogens. The industrial importance of PAHs stems from the fact that they constitute the firststage intermediates in soot formation. The objective of the present theoretical study is to understand the mechanism, energetics, and selectivity of the primary pathway in the pyrolysis of PAHs, namely the thermally induced coupling: Ar1-H $+\operatorname{Ar} 2-\mathrm{H} \rightarrow \operatorname{Ar} 1-\operatorname{Ar} 2+\mathrm{H}_{2}$; where the Ar1 and Ar2 moieties can either reside within the same molecule or belong to separate systems. Two stages of the coupling, which are the hydrogen abstraction and the recombination of aryl radicals, are investigated at the BLYP/6-311G ** level of theory. Reactions involving hydrocarbons ranging from benzene to perylene, possessing between 6 and 18 carbon atoms, are studied. Particular emphasis is paid to recognizing the relative importance of electronic and steric effects in determining the sites at which the formation of the new interring bonds occur.

\section{University of Georgia Athens, GA 30602}

\section{Department of Chemistry}

\section{Spectroscopy at Metal Cluster Surfaces Duncan, M.A. 706-542-1998

Microscopic metal clusters composed of a variety of pure component systems and metal mixtures are produced and studied in a molecular beam environment. These same methods are used to produce metal complexes, which have small molecules "physisorbed" on the surface of the metal cluster particles. Electronic spectroscopy is applied to these clusters to investigate the fundamentals of metalmetal and metal-adsorbate bonding. These studies produce vibrational frequencies, bond distances, and bond energies for metal clusters and their complexes. Recently studied systems include Ag-K, Ag-Li, Ag-Al, Ag-Ar, Ag- $\mathrm{Kr}$, and In-N $\mathrm{N}_{2}$. Studies of larger clusters focus on the metalcarbon systems known as "met-cars" clusters. In these systems, the $\mathrm{M}_{8} \mathrm{C}_{12}$ stoichiometry is formed preferentially, and a cage-like structure has been proposed to explain this preference. We are measuring dissociation products and ionization potentials for different $\mathrm{M}_{8} \mathrm{C}_{12}$ metal analogues ( $\mathrm{Ti}, \mathrm{Fe}, \mathrm{V}, \mathrm{Nb}$ ). Additional studies have identified preferential formation of $\mathrm{M}_{14} \mathrm{C}_{13}$ clusters believed to have face-centered cubic crystallite structures. The measurements of the fundamental interactions exhibited by clusters are used to evaluate their potential as models for bulk súrface chemistry and catalysis

\section{Center for Computational Quantuim Chemistry}

\section{Theoretical Studies of Elementary Hydrocärbon Species and Their Reactions Schaefer, H:F., III 706-542-2067 \\ $\$ 95,000$}

High level quantum mechanical methods are now a significant source of specific predictions concerning molecular systems that may be very important, but inaccessible to experiment. An important example is the study of molecular species and chemical reactions of fundamental importance in combustion processes. Reactions being studied using ab initio theoretical methods include the $\mathrm{C}_{2} \mathrm{H}_{5}+\mathrm{O}_{2}$ system, a number of carbyne (monovalent carbon) reactions, the varied unimolecular rearrangements of ethylnitrene, the cyclopropene-methylacetylene isomerization (for which methylvinylidene is a suggested intermediate), the glyoxal-dioxetene rearrangement, the isomerization of ketene to hydroxyacetylene, and the fragmentation of tetrahedrane. Other problems of current interest include the structure and energetics of singlet and triplet isocyanomethylene, the characterization of singlet biradical trimethylenemethane, and a theoretical examination of the hexamethyltungsten compound and its prototype $\mathrm{CrH}_{6}$.

\section{Harvard University Cambridge, MA 02138}

\section{Division of Applied Sciences}

\section{Fundamental Spectroscopic Studies of Carbenes and Hydrocarbon Radicals} Thaddeus, P.; Gottlieb, $C$. 617-495-7340

This research is dedicated to the determination of the spectroscopic constants and structure of small reactive hydrocarbons and carbenes. Many of the carbon chain radicals and carbenes observed in our laboratory are produced from acetylene-some in the same reactions that are involved in nearly all hydrocarbon fuel pyrolysis and combustion. Because the thermodynamic properties of most radical species in current models of acetylene pyrolysis are estimated by the approximation of group additivity, it is essential that the properties of smaller species be determined accurately. The recent determination of the ground state structure and prediction of the excitation energy and geometry of the low-lying excited electronic state of $\mathrm{C}_{4} \mathrm{H}$, derived from combined experimental and theoretical investigation, is the kind of information that is needed for future refinements in these models. There are also substantial uncertainties in the structures and energetics of some key radicals in kinetic models that predict the growth of hydrocarbons and formation of aromatic compounds in $\mathrm{C}_{2} \mathrm{H}_{2} / \mathrm{O}_{2}$ flames. We are in a unique position to probe this system, because many of the intermediate products have strong millimeter-wave transitions, and we can readily observe the low dipole 
moment species via ultrasensitive Fourier transform microwave spectroscopy. Recent work on two fundamental reactive molecules, HCCN and HCCCO, illustrates the powerful interplay that can occur between millimeter-wave spectroscopy and high-resolution infrared spectroscopy. This combined approach should yield spectroscopic data needed for diagnostic probing in kinetic studies and combustion environments now being developed by members of the Combustion Research Facility at Sandia National Laboratories.

\section{Department of Chemistry}

\section{Laser Spectroscopy of Hydrocarbon Radicals \\ Chen, P.; Verdine, G.L. 617-495-5323}

An extension of the thermochemical work on $\mathrm{C}_{3} \mathrm{H}_{2}$ carbenes to biradicals is underway. Biradicals of the parabenzyne type have been prepared and are now being studied for their thermochemistry and reactivity in the context of the valence bond promotion energy picture previously used for carbenes. A new chemical system for which ZEKE-PFI is ideally suited is also under study in this laboratory. The in-bicyclo[4.4.4]tetradecyl cation has a three-center, two-electron bond, and has been shown by nuclear magnetic resonance studies in solution to have a hydrogen equally between two bonding sites. By highresolution photoelectron studies of the corresponding radical, the cation at $\mathrm{cm}-1$ resolution can be accessed. It serves as the ideal model for the transition state in hydrogen atom and proton transfer reactions. Having the cation, it is possible to characterize the vibrational spectrum by photoelectron techniques and to validate the qualitative transition state models for atom transfer reaction of radicals.

\section{University of Illinois at Chicago Chicago, II 60680}

\section{Department of Chemical Engineering}

\section{Kinetics of Combustion-Related Processes at High Temperature Kiefer, J.H. 312-996-9430

The purpose of this project is to determine rates and mechanisms for fuel hydrocarbon pyrolysis and other reactions at high temperatures. The measurements are made in a shock tube (providing arbitrary, precise, and externally set temperatures) with two very high resolution laser diagnostic techniques: laser schlieren measurement of density gradients (net endothermic rate) and the new method of excimer laser flash absorption, which provides absorption profiles in the UV with $0.05 \mathrm{mi}$ crosecond resolution. Previous work included a study of the dissociation of vinylacetylene, which led to the proposal of a new mechanism for acetylene polymerization. Also, studies of large-molecule dissociation at extreme temperatures, such as the retro-Diels Alder dissociation of cyclohexene, tetrahydropyridine, and norbornene, have provided the first observations of unimolecular falloff in such dissociations. The norbornene study also offered the first measurements of incubation times in a large-molecule dissociation. Current work includes new measurements of vibrational relaxation in large molecules; dissociation, isomerization, and aromatic formation in allene/propyne; and dissociation rates in several halocarbons. A theoretical analysis of large anharmonic effects (restricted internal rotations) on the rate of dissociation of unsaturated hydrides has been developed and applied to $\mathrm{HCN}, \mathrm{C}_{2} \mathrm{H}_{2}$, and now to $\mathrm{C}_{3} \mathrm{H}_{4}$.

\section{University of Illinois at Urbana-Champaign Urbana, IL 61801}

\section{Department of Mechanical and Industrial Engineering}

\section{Investigation of Saturated Degenerate Four-Wave Mixing Spectroscopy for Quantitative Concentration Measurements \\ Lucht, R.P. 217-333-5056}

$\$ 74,363$

A combined experimental and theoretical approach is used for the development and evaluation of strategies for quantitative degenerate four-wave mixing (DFWM) concentration measurements in flames. The DFWM process is investigated theoretically by solving the timedependent density matrix questions by direct numerical integration. During the past year the effect of Doppler broadening on concentration measurements was investigated theoretically, and it was found that the DFWM reflectivity decreases significantly with increasing Doppler width. A corrected Abrams-Lind reflectivity expression was developed that shows excellent agreement with our numerical results. The effect of unequal pump intensities on DFWM lineshapes was studied, and the numerical analysis was extended to the case of forward phase-matching. Theoretical tasks will be performed during the next year on the investigation of multilevel molecular systems, the effects of multifrequency-mode lasers on DFWM signal generation, and the use of picosecond lasers for DFWM measurements in flames. Experimentally, the development of a low-pressure flame facility and DFWM apparatus has begun. The results of DFWM measurements of $\mathrm{OH}$ and $\mathrm{NO}$ over a wide range of flame pressures and stoichiometries will be compared with our theoretical calculations of DFWM signal level, saturation intensities, and detection limits. 


\section{Johns Hopkins University Baltimore, MD 21218}

\section{Department of Chemistry}

\section{Theoretical Studies of Nonadiabatic and Spin-Forbidden Processes: Investigations of Reactions and Spectroscopy of Radical Species Relevant to Combustion Reactions and Diagnostics \\ Yarkony, D.R. 410-516-4663 \\ $\$ 90,000$}

Three classes of nonadiabatic processes are currently being studied theoretically: nonadiabatic photodissociation in which an electronically excited state is produced and dissociates nonadiabatically to ground state products; spin-forbidden photofragmentation in which a nominally spin-forbidden transition is excited and leads to molecular dissociation; and spin-nonconserving ground state reactions. The fate of a photochemically excited species is an important issue in studies of pollution chemistry. In this regard it is important to elucidate both the accessible decay channels and the rate at which they are produced. Frequently electronic states are excited that can decay only nonadiabatically to ground state products. The mechanism of such decay is under consideration with particular emphasis on the effect of conical intersections on the efficiency of such processes. An initial study of the photochemical decomposition of hydroxylamine has recently been completed, and a study of isocyanic acid has been undertaken. Photoabsorption processes that change the total electron spin quantum number are frequently ignored in modeling photochemical processes. Recent experimental work suggests that this may not be the case in the photodissociation of dihydrogen sulfide. The possibility of spin-forbidden photodissociation in this system is currently under investigation. The reaction $\mathrm{N}\left({ }^{4} \mathrm{~S}\right)+\mathrm{CH}_{3}\left({ }^{2} \mathrm{~A}_{1}\right) \rightarrow \mathrm{HCN}\left(\mathrm{X}^{1} \Sigma^{+}\right)+\mathrm{H}_{2}\left(\mathrm{X}^{1} \Sigma^{+} \mathrm{g}\right)$ is spinforbidden but may play a role in hydrocarbon combustion. The feasibility of this process is currently being studied.

\section{Johns Hopkins University Laurel, MD 20723}

\section{Applied Physics Laboratory}

\section{Q-Branch Raman Scattering and Modern Kinetic Theory \\ Monchick, $L$. 301-953-6226

Rarefied gas dynamic methods of solution have been applied to the quantum kinetic equation describing light absorption, emission, and scattering. One method, the Generalized Hess Method, an analog to the BGK approximation, was applied to the HF-argon system. Using only completed collisions (i.e., on-energy-shell scattering) an essentially $a b$ initio calculation of absorption line shapes was almost indistinguishable from the experiment for low rotational level transitions in the vicinity of the line center. In the course of this study, an anomaly in the optical diffusion coefficient was elucidated and a corrected relationship established. Problems in coding the more elaborate collocation method recently published proved to be nontrivial but now have been largely solved. It is planned to study off-energy-shell scattering effects in the far wings of this system and in others where good ab initio or empirical potential energy surfaces are available. Initially, Born and half-on-energy-shell approximations will be used and eventually fully off-energy-shell scattering wave functions.

\section{Kansas State University Manhattan, KS 66506}

\section{Department of Physics}

\section{Atomic Physics with Highly Charged Ions Richard, $P$. 913-532-6783 $\$ 1,806,000$}

Investigations are made to elucidate the interactions of accelerated highly charged ions with matter. The primary goal of the project is to understand at the most basic level binary collisions involving highly charged ions and individual targets and to provide conceptual frameworks and descriptions useful in understanding interactions of highly charged ions with matter in more complex situations. The facilities used include: (a) a cryogenic electron beam ion source (CRYEBIS) capable of delivering highly charged ions up to bare argon and $\mathrm{Xe}^{46+}$ at velocities between 0.1 and 20 a.u., (b) a tandem-LINAC accelerator delivering fast beams with specific energies up to $4 \mathrm{MeV} /$ $a m u$ in charge states up to, for example, hydrogen-like chlorine, and (c) an ion-ion collision facility. The targets used include neutral gases, clusters, and solid surfaces. Quantities measured include electron spectra, both characteristic and continuum; differential and total cross sections for electron transfer, excitation, and ionization; and recoil momentum distributions. Recent experiments have focused on the role of quasi-free electron scattering in ion-atom collisions, and the relationship between electron-ion and atom-ion scattering. Saturation effects in ionization of light targets by very highly charged projectiles are studied through total cross-section measurements. An atomic hydrogen target is in use for the study of continuum electrons produced by fast ion bombardment, and a supersonic cold $\mathrm{He}$ gas jet has been recently built for high resolution recoil momentum spectroscopy in fast-ion/He collisions. The effective threshold for direct ionization in collisions of slow bare nuclei with neutral targets, in competition with well understood electron capture, has been found recently and studies of the mechanisms to account for this process are underway. The first measurements of electron capture from Rydberg targets by highly charged -projectiles has been carried out, and an effort to characterize the final states populated is in progress. The mechanisms for the breakup of molecules by fast ion bombardment and the importance of the alignment of the molecule with respect to the beam are under study. Interactions of slow, highly charged ions with graphite and mica surfaces are underway. All projects are part of a joint experimental/theoretical study, and the development of theoretical techniques, models, and conceptual frameworks is an integral part of the program. 


\section{University of Kentucky. Lexington, KY 40506}

\section{Department of Chemistry}

\section{Laser Spectroscopy and Dynamics of Transient Species \\ Clouthier, D.J. 606-257-1790

The goal of this program is to study the vibrational and electronic spectra and excited state dynamics of a number of transient sulfur and oxygen species. We have obtained high-resolution of FT-IR spectra of formyl chloride ( $\mathrm{HCOCl}$ and $\mathrm{DCOCl}$ ) and rotationally analyzed several bands to provide a detailed description of the ground state rovibrational energy levels. Interactions between vibrational levels leading to perturbations in the spectra have allowed us to obtain substantial information about a number of dark states. We have also recorded infrared spectra. of sulfine $\left(\mathrm{H}_{2} \mathrm{CSO}\right)$, reassigned the low resolution spectrum, and rotationally analyzed the $\nu_{8}$ band. In a study of the reactions of molecular fluorine with hydrogen sulfide, carbonyl sulfide, and carbon disulfide, we have detected the $\mathrm{FS}_{2}$ free radical for the first time. A vibrational and rotational analysis of the spectrum, in conjunction with high quality $a b$ initio calculations, proves that the spectrum is the $\widetilde{\mathrm{A}}^{2} \mathrm{~A}^{\prime}-\tilde{\mathrm{X}}^{2} \mathrm{~A}^{\prime \prime}$ band system of $\mathrm{FS}_{2}$ with the following structural parameters: $r(\mathrm{~S}-\mathrm{F})=1.651 \AA, \mathrm{r}(\mathrm{S}-\mathrm{S})=1.865 \AA$, and $\theta(\mathrm{FSS})=109.1^{\circ}$ in the ground state and $\mathrm{r}(\mathrm{S}-\mathrm{F})=1.642$ $\AA, r(\mathrm{~S}-\mathrm{S})=2.09 \AA$ and $\theta(\mathrm{FSS})=97.1^{\circ}$ in the excited state. We have also obtained sub-Doppler spectra of the $S_{1}-S_{0}$ systems of thioformaldehyde, shown that there are extensive interactions with the ground and excited triplet state energy levels, and concluded that there is substantial rotation-induced vibrational mixing in the ground state.

\section{Massachusetts Institute of Technology Cambridge, MA 02139}

\section{Department of Chemical Engineering}

\section{Aromatics Oxidation and Soot Formation in Flames \\ Howard, J.B. \\ 617,-253-4574 \\ $\$ 119,000$}

The oxidation of aromatics and the formation of soot in flames are being studied: with emphasis on experimental identification of important molecular species, including fullerenes, characterization of soot structure, and measurement of concentration profiles of: molecular species and soot through the reaction and post flame zones of low-pressure one-dimensional flames. The species identifications, soot structures characteristics, and net reaction rates calculated from the concentration profiles are used to test and to refine hypothesized reaction mechanisms. Proposed mechanisms of benzene oxidation are being tested; and refined as appropriate, using measured concentration profiles of radical and stable species present during benzene oxidation in flames. The research on soot formation is concerned with the particle inception or nucleation stage and the study of soot structure at all stages of growth in order to obtain mechanistic information from evidence of growth steps recorded in the structure of particles. The ultimate objective is to understand how nascent soot particles are formed from high molecular weight compounds, including the roles of planar and curved PAH and the relationship between soot and fullerenes. The objective of the research on fullerenes is to identify the range of fullerenes formed in flames, the nature of the precursor species, and the mechanisms and kinetics of the formation reactions.

\section{Department of Chemistry}

\section{Spectroscopic and Dynamical Studies of Highly Energized Small Polyatomic Molecules \\ Field, R:W:; Silbey, R.J: 617-253-1489}

Studies of intramolecular vibrational redistribution (IVR) and unimolecular isomerization have focused on the acetylene molecule $\left(\mathrm{C}_{2} \mathrm{H}_{2}\right)$ and have utilized the spectroscopic techniques of dispersed fluorescence (DF), stimulated emission pumping (SEP), and infrared-ultraviolet (IR-UV) double resonance. DF and SEP. Sensitive and selective absorption-based spectroscopic techniques, which are suited to detection and characterization, have also been under development. These techniques are based on the combination of magnetic rotation spectroscopy (MRS), which provides selectivity, to the lowest rotational levels of free radicals, and frequency modulation (FM) spectroscopy. In a collaboration with T. Sears and G. Hall at Brookhaven National Laboratory, the velocity and internal state distributions of photolytically generated free radicals (CN) were sensitivity. monitored by transient FM spectroscopy. In a collaboration with E. Eyler at the University of Delaware, the sideband phase and amplitude stability on which the sensitivity of FM spectroscopy depends was shown to be preserved when an FM'ed cw dye laser was pulse amplified and frequency tripled. The study of IVR in acetylene is based on a superpolyad model in which the initial stages of IVR are described by a few spectroscopically determined resonance parameters. The superpolyad model describes the frequency and intensity patterns in the spectrum and the rates and pathways for energy flow in a computationally simple form; which is explicitly scalable in $\mathrm{E}_{\text {vibration. }}$. The model also guides selection of initial states, accessible via IR-UV-SEP; that are optimally coupled to dynamical features such as the acetylene-vinylidene isomerization coordinate. Despite severe overlap of polyad features in the DF spectrum of acetylene, it has proven possible to "unzip" the spectrum into separate polyads. This provides a quick survey of the unimolecular dynamics at energies where breakdown of the predicted polyad patterns would signal the onset of isomerization. 


\section{University of Massachusetts at Amherst \\ Amherst, MA 01003}

\section{Department of Chemical Engineering}

\section{Probing Flame Chemistry with MBMS, Theory, and Modeling \\ Westmoreland, P.R. 413-545-1750

Elementary reactions in combustion are studied using molecular beam mass spectrometry (MBMS) of freeradical and stable species in flames, ab initio calculations of thermochemistry and transition states, new kinetics from reaction theories, and tests of mechanisms using whole-flame modeling. The present focus is on oxidation and molecular-weight growth chemistry in forming aromatics. Low-pressure propene and ethene flat flames are being studied for their $\mathrm{C}_{3} \mathrm{H}_{3}$ and $\mathrm{C}_{2} \mathrm{H}_{3}$ kinetics. One key measurement, $\mathrm{HO}_{2}$ in a lean propene flame $(\phi=0.229)$, showed that allyl $+\mathrm{O}_{2}$ does not make propadiene $+\mathrm{O}_{2}$. Meanwhile, new pathways were found for $\mathrm{C}_{2} \mathrm{H}_{3}+\mathrm{O}_{2}$, yielding $\mathrm{CHO}+\mathrm{CH}_{2} \mathrm{O}$ at low temperatures via a dioxirane intermediate but $\mathrm{C}_{2} \mathrm{H}_{2}+\mathrm{HO}_{2}$ at high temperatures via disproportionation. These predictions come from HF/6-31G* transition-state modeling by the BAC-MP4 methodology of Melius. Current experiments are on ethene and mixed $\mathrm{C}_{3} \mathrm{H}_{4} / \mathrm{LPG}$ flames.

\section{Department of Chemistry}

\section{Theory of the Dissociation Dynamics of Small Molecules on Metal Surfaces: Finite Temperature Studies \\ Jackson, B.E. 413-545-2583

Realistic theoretical models are used to examine the dynamics of some molecule-surface reactions important in catalysis. Time-dependent techniques are used to treat the necessary degrees of freedom quantum mechanically. The dissociative adsorption of molecular hydrogen and its isotopes on metals has been studied in detail. Three molecular degrees of freedom are treated quantum mechanically, and the other three classically. Dissociation probabilities were computed for many initial molecular states, and combined to stimulate molecular beam experiments. Studies have been made of Eley-Rideal processes in which a gas phase $\mathrm{H}$ or $\mathrm{D}$ atom reacts with an adsorbed $\mathrm{H}, \mathrm{D}$, or $\mathrm{Cl}$ atom. Using a simple collinear model, observed activation energies have been explained in terms of enhanced reactivity due to adsorbate vibrational excitation. A fully quantum three-dimensional calculation has been implemented, which allows for the determination of reaction cross sections and product translational and rovibrational state distributions. Detailed studies of Eley-Rideal reaction dynamics have been made for several model potential energy surfaces. The effects of isotopic substitution have been examined. A mechanism in which the incident atom becomes trapped due to collisions with adsorbed atoms has been investigated.

\section{University of Michigan Ann Arbor, MII 48109}

\section{Department of Atmospheric, Oceanic, and Space Sciences}

\section{Energy-Transfer Properties and Mechanisms \\ Barker, J.R. \\ 313-763-6239}

$\$ 110,000$

This project studies the mechanisms and properties of energy transfer involving moderate-sized molecules. A fuller understanding of highly excited molecules is obtained by a combination of experiments and modeling. In the experiments, the population distributions of the excited molecules are monitored with various techniques, including time- and wavelength-resolved IR emission and resonance-enhanced multiphoton ionization. The aim is to assemble a reliable data base and to develop a workable theoretical model for prediction of energy transfer properties. Another important objective is to determine the disposal of energy in translational, rotational, and vibrational degrees of freedom as highly excited molecules are deactivated. In the modeling effort, collisional/reaction master equation formulations are used to investigate the effects of the energy transfer properties on chemical reaction systems of interest in combustion and in other systems that experience temperature and pressure extremes.

\section{University of Minnesota Minneapolis, MN 55455}

\section{Department of Chemistry}

\section{State-to-State Dynamics of Molecular Energy Transfer \\ Gentry, W.R.; Giese, C.F. 612-625-2894

The goal of this research program is to elucidate the elementary dynamical mechanisms of vibrational and rotational energy transfer between molecules at a quantum-state resolved level of detail. Molecular beam techniques are used to isolate individual molecular collisions, and to control the kinetic energy of collision. Lasers are used both to prepare specific quantum states prior to collision by stimulated-emission pumping (SEP), and to measure the distribution of quantum states in the collision products by laser-induced fluorescence (LIF). Current activity focuses on resonant and near-resonant vibrationto-vibration energy transfer in collisions of two NO molecules.

\section{Variational Transition State Theory Truhlar, D.G. 612-624-7555 \\ $\$ 111,000$}

This project involves the development and application of variational transition state theory (VTST) and semiclassical transmission coefficients to gas-phase reactions. The work involves bold development of the theory and development of practical techniques for applying the theory to various classes of transition states and for interfacing 
reaction-path dynamics calculations with electronic structure theory and applications to specific reactions. We have developed new general strategies for obtaining potential energy surface information for reaction-path dynamics calculations, and we are developing practical methods that eliminate the need for fitting processes. The new methods are called direct dynamics. We have developed one approach to direct dynamics that we call interpolated variational transition state theory (IVTST). In this approach, we base the dynamics calculation on electronic structure calculations, including energies, gradients, and hessians, at the reactants, products, saddle point, and zero, one, or two additional points near the saddle point. Our newest direct dynamics scheme is called VTST with interpolated corrections (VTST-IC) or dual-level direct dynamics or triple-slash (/II) dynamics. This approach involves using straight direct dynamics with NDDO-SRP parameterization as a first step and augmenting it with high-level data at selected geometries along the reaction path. Applications are being made to combustion processes.

\section{National Institute of Standards and Technology, Gaithersburg Gaithersburg, MD 20899}

\author{
Chemical Science and Technology Laboratory
}

\section{Optically Driven Surface Reactions State-Resolved Probes of Surface Dynamics Cavanagh, R.R.; King, D.S. 301-975-2368

Lasers and state-resolved diagnostics are used to initiate and follow chemical processes on solid surfaces. Optical excitations allow the study both of thermal and nonequilibrium chemistries that might arise naturally during catalytic reaction and materials processing. Laser wavelengths ranging from the IR through the UV are available to initiate chemical transformations by creating thermal, adsorbate-localized, or substrate-mediated excitations. Quantum state resolved diagnostics of the reaction products allow for a better understanding of the detailed reaction mechanism(s) that follow and the dependence of reaction pathway(s) on the excitation mechanism. Previous work in this laboratory clearly demonstrated the first evidence for the importance of hot-carrier-driven chemistry on a metal surface [NO/Pt(III)], surface state-driven chemistry on a semiconductor [NO/Si(III) $7 \times 7$ ], and substrate and adsorbate quenching effects in adsorbate photolysis $\left[\mathrm{Mo}(\mathrm{CO})_{6} / \mathrm{Si}(\mathrm{III})\right]$. Current work is directed at understanding the dynamics of photostimulated oxidation of carbon surfaces and testing theoretical models of hot electron-stimulated desorption dynamics.

\section{Kinetics Database for Combustion Modeling \\ Tsang, $W$. 301-975-2507}

The computer simulation of combustion processes provides a means of making optimum use of increasing capabilities in computational power and fundamental understanding to supplement physical testing. It can therefore have important influences on design and optimization of combustion devices for energy efficiency and pollution minimization. The key ingredient for taking advantage of this technology is the availability of a reliable database of chemical kinetic and thermodynamic information bearing on all the species, stable and unstable, that are formed in the course of the reaction. This project seeks to fulfill this need through the development of a database of evaluated and estimated chemical kinetic and thermodynamic information. The procedure has been to start with the simplest of hydrocarbons, methane, and then add increasingly complex and more realistic fuel molecules containing the functional groups that are the key to their reactivity and the basis for estimation. Thus smaller saturated alkenes, alkynes, and aromatics have now been examined. An important aspect of this work is the examination of techniques for estimation. Much progress has now been made in developing an understanding of the limitations of the general procedures for extrapolation of data to the high temperatures required for combustion applications.

\section{Physics Laboratory}

\section{Spectroscopic Investigation of the Vibrational Quasi-Continuum Arising from Internal Rotation of a Methyl Group Hougen, J.T. 301-975-2379 \\ $\$ 75,000$}

This project studies the vibrational quasi-continuum in acetaldehyde, methanol, hydrogen peroxide, and related molecules because internal rotation is important in promoting intramolecular vibrational redistribution (IVR). It aims to understand: (1) torsional motion below and above the barrier, (2) traditional vibrational states, and (3) interactions involving levels with excitation of both kinds of motion. A global quantum-mechanical fit to measurement accuracy of acetaldehyde torsion-rotation levels below the barrier $\left(v_{t} \leq 2\right)$ and just above the barrier $\left(v_{t}=3\right.$ and some $\left.v_{t}=4\right)$ has been achieved, and a manuscript containing this fit together with an overview of internal rotation levels above the barrier, a discussion of torsion-rotation interactions above the barrier, and sample intensity calculations is in preparation. An analogous fit of levels below and just at the barrier for the related molecule methanol has also been completed. Work is continuing on attempts to use analytical tools from classical mechanics (e.g., Poincarẽ surfaces of section and coherent state projections) to unify understanding of torsion-rotation levels. Theoretical work has begun on developing a new vibration-torsion-rotation interaction believed to be responsible for methyl rotor enhancement of IVR rates. 


\section{University of New Orleans \\ New Orleans, LA 70148}

\author{
Department of Chemistry
}

\section{Identification and Temporal Behavior of Radical Intermediates Formed during the Combustion and Pyrolysis of Gaseous Fuel Kern, R.D., Jr. 504-286-6847 \\ $\$ 90,000$}

The role of propargyl radicals with regard to the efficient production of benzene and the subsequent high yields of soot was investigated by studying the pyrolysis of propargyl bromide. Reactant and product profiles for the decomposition were obtained over the temperature and total pressure ranges, $1310-1740 \mathrm{~K}$ and $0.20-0.34 \mathrm{~atm}$, by analyzing the reflected shock zone by time-of-flight mass spectrometry. Software was written to identify the various $\mathrm{m} / \mathrm{e}$ values and to measure their respective peak heights recorded by a high-speed transient digitizer. A parallel dissociation scheme is proposed for the initiation step: one channel produces singlet cyclopropenylidene and hydrogen bromide; the second channel yields propargyl radical and bromine atoms. The relative contributions of these two channels were determined to be equal over the temperature and presure range covered. An important observation is the twofold increase in benzene yield when hydrogen is added to the preshocked mixture. The explanation for this result is the production of additional propyne and allene and the consequent increase in the concentration of propargyl radicals. High-level theoretical calculations to determine the activation energies for the insertion reactions of singlet cyclopropenylidine and its structural isomers into hydrogen are being performed.

\section{New York University New York, NY 10003}

\section{Department of Chemistry}

\section{Accurate Polyatomic Quantum Dynamics Studies of Combustion Reactions Zhang, J.Z.H. 212-998-8412 \\ $\$ 75,000$}

New computational methods are being developed for the time-dependent approach to polyatomic combustion reactions. The new development is aimed at tackling more complex reaction systems that require a large number of basis functions in quantum dynamical approach. The proposed method (normalized angular quadrature scheme) enables one to obtain stable and accurate results without the need for large-matrix storage and large-matrix multiplications. As a result, the benchmark $\mathrm{H}_{2}+\mathrm{OH}$ and its isotopic reaction $\mathrm{HD}+\mathrm{OH}$ can now be calculated efficiently on a medium-sized workstation. Application of this new method to other combustion reactions is in progress.

\section{University of North Carolina at Chapel Hill Chapel Hill, NC 27599}

\section{Department of Chemistry}

\section{The Energetics and Dynamics of Free Radicals, Ions, and Clusters \\ Baer, T. 919-962-1580

The dissociation dynamics of energy selected ions are investigated by photoelectron photoion coincidence (PEPICO). Molecules are prepared in a molecular beam so that their internal as well as translational temperature is near $0 \mathrm{~K}$. The primary experimental information includes ionization and fragment appearance energies, and ion time-of-flight (TOF) distributions. The latter permit the measurement of dissociation rates and product energy distributions. A recent study of the butene ion dissociation has permitted the analysis of three very different dissociation reactions (the loss of $\mathrm{H} \mathrm{CH}_{3}$ and $\mathrm{CH}_{4}$ ) by a combination of $a b$ initio molecular orbital and RRKM statistical theory calculations. The aim is to develop simple methods for calculating dissociation rates with the statistical theory. The role of angular momentum in slowing down the reaction rates is also determined. Measurements of dissociation onsets have yielded new values for heats of formation of unstable species, including the $t$ butyl ion. Present experiments are designed to measure the onsets for the loss of neutral free radicals from dimer ions. Among the reactions being investigated are those that produce the neutral t-butyl free radical. These experiments are made possible by the narrow TOF distributions achieved with the cold molecular beam. It permits distinguishing dimer ions formed from the photoionization of neutral dimers from those produced by dissociative ionization of neutral trimers.

\section{North Dakota State University Fargo, ND 58105}

\section{Department of Chemistry}

\section{3. "Infrared Laser Studies of the Combustion Chemistry of Nitrogen" \\ Hershberger, J.F. 701-231-8225

The project under investigation is part of a broad effort to understand at a molecular level the detailed kinetics of combustion processes. The studies in our laboratory concentrate on radical-radical and radical-molecule chemical reactions which either form or destroy $\mathrm{NO}_{\mathrm{x}}$. Many of these reactions are important in $\mathrm{NO}_{\mathrm{x}}$ control strategies such as thermal de-NO $\mathrm{NO}_{\mathrm{x}}$ and NO-reburning. The emphasis is primarily on the quantitative determination of product branching ratios for reactions that have several possible channels. Used UV laser photolysis to inititiate these reactions, and infrared diode laser spectroscopy to probe reaction products. Some of the reactions recently studied and/or currently under study include $\mathrm{NH}+\mathrm{NO}, \mathrm{NH}+$ $\mathrm{NO}_{2}, \mathrm{NH}_{2}+\mathrm{NO}_{2}$, and $\mathrm{CH}_{2}+\mathrm{NO}$. 


\section{University of Oregon Eugene, OR 97403}

\section{Department of Chemistry}

\section{Dynamical Analysis of Highly Excited Molecular Spectra \\ Kellman, M.E. 503-346-4196 \\ $\$ 95,000$}

A framework for analysis of highly excited vibrational states of polyatomic molecules is investigated using techniques of nonlinear dynamics, especially bifurcation theory. The goal is classification of the motions of molecules excited to the regime of chaotic dynamics, and determination of patterns resulting from these, motions in experimental spectra. Several research areas are being investigated with application to species and methods of interest in combustion processes. The first is a method based on diabatic correlation diagrams applied to polyatomic spectra with chaotic dynamics, specifically, triatomics such as $\mathrm{H}_{2} \mathrm{O}$, and larger molecules such as acetylene. For the triatomics, the critical points of an effective Hamiltonian used for fitting spectra are analyzed, giving the large-scale bifurcation structure of the molecular phase space. An assignment procedure using the bifurcation analysis, in tandem with the correlation diagram procedure, is being investigated. A more phenomenological extension to acetylene is being investigated, where the bifurcation structure is harder to obtain, and at present, unknown. The final research area, with application to experimental spectra of $\mathrm{CS}_{2}$, is bifurcation and semiclassical quantum analysis of two degree-offreedom systems with such strong coupling that earlier methods of analysis of chaotic systems are inapplicable.

\section{Pennsylvania State University, University Park University Park, PA 16802}

\section{Department of Chemistry}

\section{Metal Cluster Alloys and Oxides Elucidating Structural and Electronic Effects in Governing the Reactivity and Catalytic Role of Matter in Finite Dimensions}

Castleman, A.W., Jr. 814-865-7242

Our research program is directed toward understanding catalysis at the molecular level, with the overall objective of providing the basis for the eventual designs of ones that can provide a high degree of selectivity and yield facile conversions and desired reaction products. Gurrent focus is on clusters composed of oxides, both in terms of their own innate catalytic activity as well as their use as supports of metal catalysts, and in carbides that often display considerable selectivity and represent promising materials for further development. Attention is directed to determining the influence of oxidation state, stoichiometry, and charge on various properties and reactions. During the past year new insight has been gained into the role of the electronic structure of the constituent metal atoms in terms of the reactivities of binary metal MetCars, and their influence on reactions including those of ketones, organic halides, and nitrogen oxides. Other related work on cluster reactivities has revealed the influence of degree of aggregation through studies of metals such as magnesium and various other alloys composed of copper bound to other metal atoms, and reactions of transition metal oxide clusters with small organic molecules, ammonia, $\mathrm{N}_{2} \mathrm{O}, \mathrm{H}_{2}$, and water.

\section{University of Pennsylvania Philadelphia, PA 19104}

\section{Department of Chemistry}

\section{Spectroscopy and Reactions of Vibrationally Excited Transient Molecules Dai, H.L. 215-898-5077 $\$ 110,000$}

The reactions and energy transfer properties of highly vibrationally excited molecules with chemically significant energy are characterized through a combination of laser and Fourier transform spectroscopic techniques. These experiments generate detailed information on the behavior of a highly excited molecule during a collision that is useful to the understanding of how vibrational energy drives chemical reactions. We have so far characterized the collisional deactivation of $\mathrm{NO}_{2}$ excited with as much as 22,000 $\mathrm{cm}^{-1}, \mathrm{CS}_{2}$ and $\mathrm{SO}_{2}$ up to $32,000 \mathrm{~cm}^{-1}$, and pyrazine with $52,000 \mathrm{~cm}^{-1}$ energies. Of the many unanticipated observations is the identification of intramolecular electronic coupling in enhancing the collisional quenching of highly vibrationally excited molecules. Results show that there is a dramatic increase in the average energy removed per collision for $\mathrm{NO}_{2}$ excited above $\sim 10,000 \mathrm{~cm}^{-1}$ and for $\mathrm{CS}_{2}$ above $\sim 26,000 \mathrm{~cm}^{-1}$. These energies correspond to the origins of the lowest excited $\tilde{A}^{2} B_{2} / \tilde{B}^{2} B_{1}$ states of $\mathrm{NO}_{2}$ and the lowest excited $\mathrm{R}^{3} \mathrm{~A}_{2}$ state of $\mathrm{CS}_{2}$. Mixing between these excited electronic states with the ground electronic state enhances collisional relaxation by allowing the electronic transition dipole to contribute to collisional energy transfer.

\section{Intermolecular Interactions of Hydroxyl Radicals on Reactive Potential Energy Surfaces \\ Lester, M.I. $215-898-4640$ \\ $\$ 105,000$}

This program is focused on the characterization of the interaction potentials between the hydroxyl radical in its ground $\mathrm{X}^{2} \Pi$ and excited $\mathrm{A}^{2} \Sigma^{+}$electronic states and various collision partners. This is accomplished by aggregating the collision partners in a weakly bound complex and probing these binary complexes through spectroscopic measurements and half-collision dynamical studies. The $\mathrm{OH}-\mathrm{Ar}$ system has proven to be a prototype for examining the interaction potential of an open-shell system since it is amenable to experimental investigation and theoretically tractable from first principles. In addition, recent studies of $\mathrm{OH}-\mathrm{H}_{2} / \mathrm{D}_{2}$ complexes are providing a detailed picture of the $\mathrm{OH}\left(\mathrm{A}^{2} \Sigma^{+}, \mathrm{X}^{2} \Pi\right)+\mathrm{H}_{2} / \mathrm{D}_{2}$ potential energy surfaces and the reaction dynamics taking place on these surfaces. Current work is aimed at understanding the $\mathrm{OH}\left(\mathrm{A}^{2} \Sigma^{+}\right)+$ 
$\mathrm{N}_{2}$ system and the origin of the surprisingly large quenching cross section measured for this collision partner.

\section{Princeton University Princeton, NJ 08544}

\section{Department of Chemistry}

\section{Analysis of Forward and Inverse Problems in Chemical Dynamics and Spectroscopy Rabitz, $H, A$. 609-258-3917 $\$ 105,000$}

This research is concerned with fundamental issues in molecular science and practical solutions in chemical kinetics engineering problems. The first part of the research aims to develop and apply new algorithms to optimally draw on $a b$ initio quantum chemistry calculations and available laboratory data in order to gain a quantitative understanding of the effect of molecular interactions on spectroscopic, dynamical, and kinetic events, Specifically, mathematical and numerical tools based on functional sensitivity analysis, inverse problem theory, and reproducing kernel theory have been developed for identifying the relationship between potential energy surfaces and observables (forward analysis), and for constructing physically acceptable potential energy surfaces from either laboratory data or ab initio calculations. The second part of the research aims to develop effective dimension reduction methods for constructing simplified, yet accurate, combustion models. Here a recently formulated algebraic-based method, along with nonlinear perturbation theory, has been employed to develop rigorous and efficient nonlinear lumping schemes for combustion model simplification.

\section{Department of Mechanical and Aerospace Engineering}

\section{Comprehensive Mechanisms for Combustion Chemistry: An Experimental and Numerical Study with Emphasis on Applied Sensitivity Analysis Dryer, F.L. 609-258-5206 \\ $\$ 110,998$}

This program addresses improving understanding of combustion chemistry through experimental flow reactor studies in the temperature range $550-1200 \mathrm{~K}$, the pressure range 1-20 atmospheres, and with characteristic reaction times from $10^{-2}-3$ seconds. Through the use of techniques based on elemental gradient-feature sensitivity and path analyses, computations are performed to obtain elementary rate information and to develop and study comprehensive chemical kinetic mechanisms. Elementary kinetic data are obtained from perturbation studies of the $\mathrm{CO} / \mathrm{H}_{2} /$ oxidant reaction system by small amounts of hydrocarbons and/or hydrocarbon oxygenates. Of special interest here are the reactions of $\mathrm{HO}_{2}$ with $\mathrm{CH}_{3}$ and other species. Reaction systems of interest include those for pyrolysis and oxidation of simple oxygenates (especially formaldehyde and acetaldehyde), simple olefins (especially ethene), and ethane. The research emphasizes the extension of the present knowledge, based on reaction mechanisms of these small molecules, to pressures and temperatures where the reaction of radicals with oxygen and the reactions involving $\mathrm{RO}_{2}$ and $\mathrm{HO}_{2}$ are important.

\section{Aromatic-Radical Oxidation Kinetics Glassman, $I, ;$ Brezinsky, $K$. 609-258-5199 \\ (18 months)}

Elucidation of the details of the oxidation of the aromatic ring of monocyclic aromatic species, key elements in automotive and industrial fuels, has been the main focus of this research effort. Special attention has been focused on the oxidation of $\mathrm{C}_{5}$ species, in particular cyclopentadiene (CPD), that early work in this laboratory has shown to play an important role in the sequence of steps that bridge the opening of the aromatic ring and the eventual production of hydrocarbon combustion projects. Modelling efforts have been partially successful in defining the detailed steps in benzene and toluene oxidation. However, the models have had only limited success in predicting experimental results from flow reactors, flame speed experiments, shock tubes, etc. The main difficulty appears to be with $C_{5}$ reactions and their rates. Extensive chemical analysis and flow reactor experimentation has resolved the dilemma of early $\mathrm{CO}_{2}$ during cyclopentadiene oxidation. these efforts have revealed many interesting chemical and catalytic effects peculiar to cyclopentadiene. Contamination of the reactor tube by iron coatings was found to have catalytic effects under certain operating conditions. Detailed experiments have revealed that operation at higher temperatures and under lean conditions, the homogeneous gas phase oxidation of cyclopentadiene can be readily evaluated. Interesting data are now being obtained and the initial results appear to be reasonable for understanding and developing models of the cyclopentadiene oxidation process.

\section{Rice University Houston, TX 77251}

\section{Department of Chemistry}

\section{Infrared Absorption Spectroscopy and Chemical Kinetics of Free Radicals Curl, R.F., Jr.; Glass, G.P.} 713-527-4816

This research is directed at the detection, monitoring, and study of the chemical kinetic behavior by infrared absorption spectroscopy of small free-radical species thought to be important intermediates in combustion. In order for soot to be formed in a flame, it is necessary that aromatics be present. For flames of nonaromatic fuels, the production of aromatics in the flame may be initiated by the recombination of propargyl $\left(\mathrm{HCCCH}_{2}\right)$ radicals. Others have proposed that propargyl is produced by the reaction of singlet methylene $\left({ }^{1} \mathrm{CH}_{2}\right)$ with acetylene $(\mathrm{HCCH})$. This research has confirmed this proposal by direct observation by infrared spectroscopy of the propargyl produced by the reaction between singlet methylene and acetylene. Further, the rate constant for this reaction has been measured by comparing the intensity of the propargyl infrared absorption with that of $\mathrm{CH}_{3}$ produced by a competing reaction of ${ }^{1} \mathrm{CH}_{2}$ with $\mathrm{H}_{2}$ or with $\mathrm{CH}_{4}$. The rate constant for production of propargyl is found to be $2.3(7) \times 10^{-10} \mathrm{~cm}^{3}$ $\mathrm{sec}^{-1}$ at room temperature. Better calibration measurements to improve the accuracy are now in progress 


\section{University of Rochester Rochester, NY 14627}

\section{Department of Chemistry}

\section{Low-Energy Ion-Molecule Reactions and Chemiionization Kinetics \\ Farrar, J.M. \\ 716-275-5834 \\ $\$ 94,000$}

Crossed ion beam/neutral beam reactive scattering experiments are being performed with the goal of using energy disposal measurements and angular distributions to extract dynamical information on collision mechanics and features of the potential surface mediating the reaction. Attention has been focused on the proton and hydrogen atom transfer reactions of $\mathrm{O}^{-}$with $\mathrm{HF}, \mathrm{D}_{2}$, and $\mathrm{H}_{2}$, where vibrational state resolution of the products has been accomplished. Over the collision energy range from 0.40 to $0.60 \mathrm{eV}$, the $\mathrm{O}^{-}+\mathrm{HF}$ system shows an increasing tendency to partition excess reagent translation into product translation. Such behavior represents a transition toward repulsive energy release as the collision system samples the low-energy repulsive wall with both $\mathrm{O}-\mathrm{H}$ and $\mathrm{H}-\mathrm{F}$ bonds compressed. In the $\mathrm{O}^{-}+\mathrm{D}_{2}$ and $\mathrm{O}^{-}+\mathrm{H}_{2}$ systems, product vibrational states of $\mathrm{OD}^{-}$up to $\mathrm{v}^{\prime}=3$ and $\mathrm{OH}^{-}$ up to $\mathrm{v}^{\prime}=2$ are resolved. The reaction dynamics are direct at all energies, with available energy partitioned preferentially into product vibration, particularly for forward scattered products. Interesting and unusual rapid variations in the product state angular distributions near a collision energy of $0.30 \mathrm{eV}$ show anomalous behavior that may yield detailed information on the potential energy surface near the transition state. A series of experiments on $\mathrm{O}^{-}+\mathrm{HF}, \mathrm{H}_{2} \mathrm{O}$, and $\mathrm{NH}_{3}$, in which vibrationally excited molecules are prepared by laser excitation is planned.

\section{University of Southern California Los Angeles, CA 90089}

\section{Department of Chemistry}

\section{Reactions of Carbon Atoms Using Pulsed Molecular Beams \\ Reisler, $H$. 213-740-7071

The reaction dynamics of carbon atoms in their ground state are being studied using crossed pulsed molecular beams. A beam of carbon atoms is prepared by laser ablation of graphite and crosses a molecular beam containing a second reactant. Products are detected state-selectively. The effects of translational and internal energy on enhancing reactivity are being studied. The role of translational energy on the product state distribution in the reaction of atomic carbon with nitrous oxide has been examined. The newly formed CN bond is highly internally excited, and its excitation is not affected significantly by translational energy. In contrast, the "old" NO product is formed internally cold at low translational energies, and its vibrational and rotational energies increase with increasing collision energy. These results indicate a direct reaction mechanism. Translational energy also enhances endoergic reactions of atomic carbon with $\mathrm{H}$-containing molecules such as methanol and hydrogen bromide. The effect of vibrational energy on reactivity is being studied using infrared radiation to excite the reactants to overtone and combination bands. In addition, a new pulsed radical source has been constructed for studies of reactions of free radicals.

\section{The Stabilization Theory of Dynamics} Taylor, H. S. 213-740-4112

The stabilization method and related $\mathrm{L}^{2}$ methods of continuum processes are being developed and applied to predicting the properties of chemical reactions that are of great importance in flames, atmospherics, and environmental control. The methods being developed are designed to simulate in detail such reactions. They are also designed to enable the researcher to answer the questions as to "why" the reactions act quantitatively as studied. Interpretation is an important part of the effort. New mathematical methods are being developed that efficiently compute not only the cross sections for the various reactions under study, but also quantities such as densitydensity correlation functions, which yield insight into mechanism. Much time is spent creating both serial and parallel programs to put into practice the new mathematical methods developed to treat these problems. The short-lived reaction intermediate states in the reaction $\mathrm{O}_{2 t}$ $+\mathrm{H} \rightarrow \mathrm{OH}+\mathrm{O}$ are being studied by the stabilization methods as aided by our new Chebyshev series method of computing bound and scattering states. More diverse systems of greater complexity and dimensionality will be investigated. The mathematical methods developed here are being and can continue to be "spun off" and transferred to the problem of simulating electron devices of such small scale that quantum effects dominate the operation of nanometer size switches, diodes, and lasers. Such nanostructure technology may become the electronics industry of the future.

\section{Reactions of Small Molecular Systems Wittig, $C$. 213-740-7368 \\ $\$ 110,000$}

This program examines fundamental processes relevant to hydrocarbon combustion. The main issues are mechanisms, rates, dynamics, etc. for unimolecular and bimolecular reactions. Emphasis is placed on a sophisticated experimental technique: high-n Rydberg time-of-flight (HRTOF) spectroscopy. Specialized laser equipment is also used: a tunable, high-energy, narrowbandwidth parametric oscillator (to vibrationally excite reactants) and a femtosecond resolution pump-probe system. Ties are maintained with theoretical efforts dealing with the same systems. The scientific goals include (1) experimental studies of the HOCO radical intermediate: microcanonical $k(E)$ values from threshold to several thousand wavenumbers above threshold; an accurate measurement of the HOCO well depth; $\mathrm{CO}_{2}$ vibrational distributions from the $\mathrm{H}+\mathrm{CO}_{2}$ channel, which helps to establish transition state properties and the barrier height; overtone spectra below and above threshold; resonances above threshold; (2) unimolecular decomposition of radicals such as $\mathrm{CH}_{3} \mathrm{O}$ and $\mathrm{C}_{2} \mathrm{H}_{5}$ : spectra below and above threshold; product vibrational resolution via HRTOF, which helps establish transition state properties; and (3) photodissociation studies using the HRTOF method: development of a source of monoenergetic atomic hydrogen 
at energies betweeen 4000 and $8000 \mathrm{~cm}^{-1}$; bond dissociation energies for radicals and stable molecules; preparation of radical-molecule complexes such as $\mathrm{CH}_{3} \mathrm{O}-$ $\mathrm{C}_{2} \mathrm{H}_{2}$ by photoejecting hydrogen from a weakly bound complex.

\section{Stanford University Stanford, CA 94305}

\section{Department of Mechanical Engineering}

\section{Spectroscopy and Kinetics of Combustion Gases at High Temperatures \\ Hanson, R.K.; Bowman, C.T. 415-723-1745 \\ $\$ 135,000$}

This program involves two complementary activities: (1) development and application of $\mathrm{cw}$ laser absorption methods for sensitive detection of species and measurement of fundamental spectroscopic parameters at high pressures and temperatures; and (2) shock tube studies of chemical reactions relevant to combustion. Species currently under investigation in the spectroscopic portion of the research include $\mathrm{OH}, \mathrm{HO}_{2}$, and $\mathrm{CH}_{3}$. Shock tube studies of reaction kinetics are focused on the reactions $\mathrm{C}_{2} \mathrm{H}_{6} \rightarrow 2 \mathrm{CH}_{3}, \mathrm{H}+$ $\mathrm{O}_{2} \rightarrow \mathrm{HO}_{2}$, and $\mathrm{CO}+\mathrm{OH} \rightarrow \mathrm{CO}_{2}+\mathrm{H}$.

\section{State University of New York at Stony Brook Stony Brook, NY 11794}

\section{Department of Chemistry}

\section{Ionization Probes of Molecular Structure and Chemistry \\ Johnson, P.M. \\ 516-632-7912 \\ $\$ 90,000$}

Ionization processes in intense wavelength tunable laser fields are being used to investigate the spectroscopy and photochemistry of ions and molecules. Resonant multiphoton ionization, laser threshold ionization spectroscopy, and multiphoton fluorescence spectroscopy provide sensitive tools for the detection of transient species and for examining the excited state structure and dynamics of molecules. These methods also provide means of the detection and identification of minute quantities of molecular species in difficult environments such as the mixtures produced in combustion reactions. A newly developed primary tool in these studies is mass analyzed threshold ionization spectroscopy, which provides high resolution ion vibrational spectra of selected molecules. In this technique the various ionization thresholds that mark the energy states of an ion in transitions from a neutral state are measured by using the fact that very highly excited neutral states near each threshold can be ionized by an electric field. The ions produced in this way are sent through a mass spectrometer so the optical spectrum of each mass is obtained with high sensitivity. This technique is being applied to the detection and identification of the products of the photochemical oxidation reactions of mixed clusters systems such as aromatics and $\mathrm{O}_{2}$ or $\mathrm{N}_{2} \mathrm{O}$.
University of Utah Salt Lake City, UT 84112

\author{
Department of Chemistry
}

\section{Thermochemistry and Reactivity of Transition-Metal Clusters Armentrout, P.B. 801-581-7885}

The objective of this project is to obtain information regarding the thermodynamic properties of transition metal clusters, their binding energies to various ligands, and their reactions by using a metal cluster guided ion beam mass spectrometer and a cluster ion photodissociation spectrometer. Recent progress includes studies of the reactions of vanadium, chromium, and iron cluster ions with $\mathrm{D}_{2}$. Metal cluster monodeuteride ions are observed as the major product for all systems. For larger clusters, the dideuteride cluster ions are also observed. Analysis of the kinetic energy dependence of the cross sections for these products yields cluster ion-deuterium atom bond energies. Analysis of this provides information regarding the geometric and electronic structures of the bare clusters. Studies of the reactions of iron and vanadium clusters with $\mathrm{O}_{2}$ have also been obtained and should yield cluster monoxide and dioxide bond energies.

\section{University of Washington Seattle, WA 98195}

\section{Department of Chemistry}

\section{Atomic Probes of Surface Structure and Dynamics \\ Jonsson, H.; Heller, $E$. 206-685-1804 \\ $\$ 109,000$ \\ (24 months)}

This project involves theoretical calculations of atomic and molecular interactions with surfaces. A wide range of techniques are used and developed to address the various aspects of the problem. A reversible work-based transition state theory has been developed to estimate transition rates in multidimensional systems, including quantum degrees of freedom. This has been applied to a fully quantum and thermally averaged $\mathrm{H}_{2}$ molecule on a $\mathrm{Cu}(110)$ surface. Also, a method for finding the minimum energy path for a transition in a multidimensional system has been developed and applied to this problem. The scattering of atoms, in particular, $\mathrm{H}_{\mathrm{e}}$ atoms, from overlayers and defects on metal surfaces, such as steps and adatoms has been studied by time-independent scattering calculations as well as time-dependent Gaussian wavepacket calculations. The calculations aid the interpretation of experimental measurements taken by others on atomic structure and kinetic processes at surfaces, such as diffusion, annealing, and growth. A new semi-classical technique, "Cellular Dynamics," is being developed and will allow faster and more accurate atom scattering calculations, not only of diffractive scattering, but hopefully also of the selective adsorption resonances, which can provide very useful information about atom surface interactions. 


\section{Üniversity of Wisconsinin at Madison Madisón, Wi 53706}

\section{Department of Chèmistry}

\section{Vibrätional State Control of Phótódissóciatiòn \\ Crim, F. $\bar{F}$. \\ 608-263-7364}

The fundamental and practical importance of highly vibrationally excited molecules in combustion processes, atmospheric chemistry, plasmas, and a host of other environments motivates their detailed experimental investigation. This research uses a combination of laser excitation to prepare highly vibrationally excited molecules with single-quantum-state resolution, and spectroscopic detection to monitor the excited molecule or its decomposition product, in studies of the unimolecular reaction; photodissociation, and bimolecular reaction dynamics of vibrationally energized molecules. A collection of state preparation and detection techniques gives these measurements broad scope. The excitation approaches are vibrational overtone excitation, stimulated emission pumping, and stimulated Raman excitation, and the detection methods are UV and VUV laser-induced fluorescence and laser-induced grating spectroscopy. By selectively preparing vibrational states and subsequently dissociating or reacting them, these experiments explore normally inaccessible regions of both the ground and electronically excited potential energy surfaces. These approaches have even achieved laser control of the course of a chemical reaction. The experiments provide new insights into the structure and dynamics of vibrationally excited molecules, which play an important role in fundamentally and practically important processes.

\section{Spectroscopy Studies of Methyl Group Internal Rotation and of Organic Free Radical Vibration \\ Weisshaar, J.C. 608-262-0266

One long-term goal of this work is to develop new techniques for measuring vibrational spectra of polyatomic neutral-free radicals. Toward that end, an optical parametric oscillator (OPO) has been constructed based on the crystal KTP and pumped by the second harmonic of an injection-seeded Nd:YAG laser to provide tunable near-IR. Testing of this device is underway. IR absorption will be detected by depletion of laser-induced fluorescence from $\nu$ $=0$ radicals formed by photolysis of suitable precursors upstream in a pulsed nozzle expansion. In addition, work will be initiated to address the spectroscopic problem of internal rotation of methyl and silyl groups attached to aromatic rings. Statistical modeling of chemical reactions of molecules that include methyl rotors requires good intuition about whether internal rotation is nearly free or hindered. Internal rotors present relatively tractable examples of noncovalent interactions that determine the energetics of different molecular conformations as well. Such rotors also accelerate intramolecular vibrational redistribution and alter photochemical pathways. From a combination of spectroscopic experiments and ab initio calculations, an understanding is developing of the pattern of rotor barriers vs. electronic state and also the effects of chemical substitution at different locations on the benzene ring. The result is a growing intuition about the mechanism by which noncovalent interactions within a molecule determine the potential energy function for internal rotation.

\section{Atomic Physics}

\section{California Institute of Technology Pasadena, CA 91125}

\section{Division of Physics, Mathematics, and Astronomy}

\section{Spectroscopy with Nonclassical Light Kimble, $H . J$. 818-395-8340

Radiative interactions of simple atomic systems with light, which is manifestly quantum or nonclassical in its characteristics, will be investigated. Examples of nonclassical radiative fields include squeezed states of light and states that exhibit photon antibunching and subPoissonian photon statistics. Frequency tunable sources of such fields are being developed in the laboratory with the objective of exploring a variety of applications in atomic spectroscopy. From the perspective of quantitative spectroscopic analysis, squeezed light offers the potential for enhanced sensitivity beyond the usual quantum limit set by the vacuum fluctuations of the electromagnetic field (the so called coherent-state or shot-noise limit). However, in addition to the possibility for improved measurement sensitivity, the coupling of atoms to squeezed, antibunched, or sub-Poissonian fields should lead to fundamentally new atomic radiative processes relevant to diverse problems in optical physics, including resonance fluorescence and laser operations. Apart from the scientific objectives, the research program should make important contributions to a number of areas of technological significance, including spectroscopic detection of weakly absorbing or transient species and efficient harmonic generation from the near infrared into the blue.

\section{California State University, Fullerton Fullerton, CA 92634}

\section{Department of Physics}

\section{Few-Body Coulomb Systems Feagin, J.M.} 7.14-773-3366

Recent work has been motivated by a long-standing pursuit to propagate : a wavepacket near the threshold for few-body breakup by solving the time-dependent Schrodinger equation numerically but directly on a finite grid. Sets of collective coordinates especially suited for the process have been devised, and the Wannier description is presented in terms of a familiar theory of small oscillations that emphasizes Coulomb instabilities. The Wannier description of two-electron threshold escape has been extended to fourth-order internal coordinates. The Wannier threshold law has been rederived and corrections that 
agree with data on both hydrogen and helium to 10 electronvolt above threshold have been made. This fourthorder S-wave description to positron impact, including use of higher partial waves has been generalized to calculate differential cross sections in energy and detector angle of the electron pair. The fourth-order wavefunction will be used to establish the boundary conditions of a numerical wavepacket program.

\section{Clark Atlanta University Atlanta, GA 30314}

\section{Department of Physics}

\section{Studies of Photon and Electron Interactions with Atoms and Ions Msezane, A.Z. 404-880-8663}

Electron and photon interactions with ground and excited atoms, ions and molecules of importance in lasers, astrophysical, and laboratory fusion plasmas are studied to understand the underlying physics, and the results are used to guide measurements. The analytic properties of the electron differential cross sections (DCSs) in the complex K-plane together with Padẽ approximations are used to recompute the inelastic and elastic cross sections and optical oscillator strengths. Small-angle electron DCSs for optically allowed transitions in atoms, ions, and molecules are determined using a universal function derived recently to delineate dynamic effects as a function of impact energy. The R-matrix method is used with extensive configuration interaction target wave functions that account for correlation and core-polarization effects. It is used to calculate integral and differential cross sections. Detailed multistate photoionization calculations are performed for ground and innershell excited states to investigate and understand recent predictions and experimental measurements demonstrating strong enhancement of shake-up satellites and many-electron effects in sodium and potassium.

\section{University of Colorado Boulder, CO 80309}

\section{Department of Physics}

\section{Physics of Correlated Systems Greene, C.H. 303-492-4770}

Quantum mechanical calculations are being carried out for a number of highly correlated systems that have resisted theoretical efforts to this day. This research effort is attempting to understand the behavior of open-shell atomic systems in regimes that are not readily handled by conventional theoretical methods. Substantial headway has allowed calculating spectra of great complexity even for transition metal atoms such as titanium. A calculation is underway on very high two-electron excitations of the negative ions of lithium and sodium. Studies of strongly correlated ions will continue as for other two- and threeelectron species that continue to pose extreme difficulties for atomic theory. A new project is underway to explore the interactions of three identical bosons. This should elucidate some issues relevant to current experimental efforts to achieve Bose-Einstein condensation.

\section{University of Connecticut Storrs, CT 06269}

\section{Department of Physics}

\section{Experiments in Ultracold Collisions Gould, P.L. 203-486-2950}

The goal of this project is to investigate atomic collisions at temperatures below $10^{-3} \mathrm{~K}$. These extremely low energy collisions exhibit several unique features: large collisional deBroglie wavelength, sensitivity to long-range interatomic potentials, and the possibility of spontaneous decay during the course of the slow collision. Applications of laser cooling require understanding and/or control of these collisions since they are an important loss mechanism for high density samples. Rubidium atoms are cooled with diode laser light, then compressed and confined in a magneto-optical trap. Inelastic trap loss collisions are observed by measuring the ejection rate of atoms from the laser trap. Having carefully characterized the trapped sample, a detailed investigation of the conditions necessary for escape from the trap is now underway. The important role of excited-state hyperfine structure in these collisions has been recently examined and the temperature dependence of the collisional rate has been measured. A dramatic reduction of the loss rate at low temperatures has been observed. Efforts to suppress inelastic collisions with laser light have been successful. Experiments are also underway to directly detect, via state-selective ionization, products of specific collisions such as those which change fine-structure state.

\section{Plasma Density and Field Effects on Atomic Reactions \\ Hahn, Y. 203-486-4469}

A systematic theoretical study of the plasma environmental effects on atomic collision rates for high temperature fusion plasma is carried out. The atomic reaction rates modified by the plasma field are useful for plasma modelling and diagnostics. A comprehensive theory of spectral intensities and broadening has been formulated, based on the conventional pressure broadening theory and the rate equation approach. It involves three main steps: (a) an effective plasma potential $\nabla_{p}(t)$ is constructed, which contains all the 'slow' part of the plasma perturbations and depends explicitly on the plasma parameters, such as the plasma density and temperature; (b) the ionic states distorted by $V_{p}$ are generated, in the quasi-adiabatic approximation, and the various atomic rates of the 'fast' perturbations are evaluated using these distorted states, and (c) finally, a set of discrete-continuum form of coupled rate equations is constructed, which provides the population density of ionic excited and charge states. The theory has been applied in detail to hydrogen plasmas and will be extended to include impurity ions, e.g., ions of $\mathrm{C}, \mathrm{Si}$, and $F$. In the course of this study, a phenomenon of momentum coherence effect was discovered, in which the time-dependent external field can coherently interfere 
with moving electrons to enhance rates of processes in which they participate. The experimental consequences of such enhancement are being estimated, and its possible application to the tokamak divertor cooling problem is being addressed. The calculation is being completed of the plasma-distorted rates for ions important for fusion plasmas.

\section{Georgia Institute of Technology Atlanta, GA 30332}

\section{School of Physics}

\section{Statistical Fluctuations in Lasers Roy, $R$. 404-894-5265}

$\$ 93,000$

Phase-locking of two Nd lasers mutually coupled through overlap of their electric fields was demonstrated in a series of experiments and showed that the chaotic intensity fluctuations of these lasers can be synchronized. A novel scheme to digitally encode information on the chaotic output of a modulated laser and decode it with a synchronized receiver laser was developed. Experiments to test such a scheme are in progress. A new instability was discovered, first predicted theoretically in 1993, in the operation of coupled lasers for a range of coupling coefficients. This is directly relevant to the fabrication of semiconductor and solid state laser arrays, a topic of much technological interest. Experiments on erbium doped fiber lasers have shown a phase transition-like behavior at threshold and indicate that a new mechanism may contribute significantly to the observed intensity fluctuations. These lasers are unique in that several thousand modes operate simultaneously, and the coupling between these modes due to nonlinear interactions such as four wave mixing is instrumental in determining their stability characteristics. A theoretical model (due to Kuramoto) of coupled nonlinear oscillators is being investigated to explain the measurements.

\section{Harvard University Cambridge, MA 02138}

\section{Harvard-Smithsonian Center for Astrophysics}

\section{Theoretical Investigations of Atomic Collisions \\ Dalgarno, $A$. \\ 617-495-4403

Collisions at ultralow temperatures are under study. A comparison between semiclassical and quantal treatments demonstrates the existence of a quantum suppression effect. It has been shown that the scattering length in elastic collisions is manifested directly in the variation with frequency of the photoassociation fluorescence spectrum of cold times. Double photoionization of excited singlet and triplet metastable states of the helium isoelectronic sequence is being explored. Detailed calculations of the long-range forces between excited alkali metal atoms are also in progress.

\section{Kansas State University Manhattan, KS 66506}

Department of Physics

\section{Atomic Physics of Strongly Correlated Systems \\ Lin, C.D. \\ 913-532-1617 \\ $\$ 154,000$}

This project aim at the understanding the behavior of Coulomb three-body systems in atoms and molecules and includes study of excitation and rearrangement collision processes. Using hyperspherical coordinates, collisions between positrons and atoms are investigated to calculate excitation and positronium formation cross sections. The ionization cross sections are also being calculated. The hyperspherical coordinates are also being used to study triply excited states of three-electron atoms and ions. Transitions involving one and two electrons in excitation, ionization, and charge transfer processes in ion-atom collisions are also being investigated over a broad energy range.

\section{University of Kansas \\ Lawrence, KS 66045}

\section{Department of Chemistry}

\section{Atomic Physics in Strong Fields Chu, S.I. 913-864-4094}

$\$ 96,000$

New ab initio formalisms are being used to examine (1) angular distributions and partial rates for multiphoton ionization of atoms in intense fields; (2) laser-induced chemical bond "softening" and "hardening" and stabilization of molecules in intense one- and two-color laser fields; and (3) Rydberg atom multiphoton dynamics in half-cycle laser pulses. In this context, particular attention is given to above threshold ionization of $\left(h^{-}\right)$in intense laser fields. A new complex scaling generalized pseudo spectral method is introduced for the discretization and solution of the non-Hermitian Floquet Hamiltionians derived for this purpose. In addition, a novel stationary formulation for the treatment of general time dependent Hamiltionians is used to study the effects of laser pulses on multiphoton processes.

\section{University of Kentucky Lexington, KY 40506}

\section{Department of Physics and Astronomy}

\section{The Dynamics of Weakly-Bound States in Collisions and Fields Cavagnero, M.J.; Harmin, D.A. 606-257-6733

Several processes associated with the dynamics of weakly bound atomic electrons in time-dependent fields are investigated. A theory of the time evolution of state amplitudes described by a complex web of interacting energy levels is developed and applied to the experimental state detection 
technique of selective field ionization. A new Floquet approach to inelastic ion-atom collisions is applied to distant collisions of Rydberg atoms with charged projectiles, processes that are important in plasma environments. A reformulation of the problem of weakly bound electrons in dynamic two-center fields will be made to extend multichannel quantum-defect theory to time-dependent processes, A study of electron capture and ionization in ion collisions with circular and elliptic Rydberg atom targets will examine fundamental three-body processes in or near the correspondence principle limit.

\section{Coherent Excitation of Autoionizing Resonances \\ Martin, N.L.S. \\ 606-257-5840 \\ $\$ 77,600$}

To investigate fundamental interference effects in the electron impact ionization of atomic cadmium, $(e, 2 e)$ spectroscopy is being used. For small scattering angles, these interference effects are due to the coherent excitation of $J=0,1,2$ autoionizing levels and continua. The experiments probe both the magnitude and phase of excitation amplitudes. Experiments carried out at small momentum transfer have shown that plane wave Born approximation calculations using pseudo-relativistic Hartree-Fock wavefunctions, which might be expected to describe accurately the ionization process under these conditions, incorrectly predict both the magnitudes and phases of these complex amplitudes. The experimental phase discrepancies are similar to other workers' calculated phase differences between the Coulomb-Born and Plane Wave Born approximations, and the experimental magnitude ratios are about a factor of two different from the calculated values. Experiments are now in progress to observe the interference effects at larger scattering angles. Measurements to date lead to the somewhat surprising conclusion that the plane-wave Born approximation predictions get better, rather than worse, with increasing scattering angle. It is found that more partial waves are required to describe the ionization process; calculations have been carried out for $J=0$ to 7 . One interesting prediction of the Born calculations is currently being experimentally investigated, viz., for a momentum transfer of 1 a.u. the octupole transition to the $J=3$ levels of the main autoionizing configuration should become comparable in importance to the dipole transition to the $\mathrm{J}=1$ levels.

\section{University of Louisville Louisville, KY 40292}

\section{Department of Physics}

\section{Hydrogen Atom and Molecule Collisions Kielkopf, $J$. 502-852-5990

Radiative collisions involving excited states of atomic hydrogen are being studied with optical, vacuum ultraviolet, and laser spectroscopy. The source of hydrogen atoms for these experiments is the plasma produced when a Q-switched laser is focused into $\mathrm{H}_{2}$. Time-resolved shadowgraph and schlieren images are combined with spectroscopic measurements of temperature and electron density to yield a model for the development of the plasma and the state of the gas during and after the shock wave produced by the incident laser energy. Well-defined conditions in a dense atomic gas are selected by varying the time delay from breakdown. Current work includes measurements and theoretical analyses of $\mathrm{H}-\mathrm{H}$ and $\mathrm{H}-\mathrm{H}^{+}$ collisions at densities high enough to observe several atoms interacting simultaneously with the radiator, radiative collisions of unexcited atomic $\mathrm{H}$ with excited alkali atoms, and bound-free continua from radiative dissociation of $\mathrm{H}_{2}$ and $\mathrm{OH}$.

\section{Michigan Technological University Houghton, MI 49931}

\section{Department of Physics}

\section{Theoretical Hyperfine Structure Constants for Transition-Metal Atoms and Ions Beck, D.R.

Accurate values for properties of transition metal atoms and positive ions important for plasma diagnostics, catalysis, deep level traps in semiconductors, hydrogen storage, astrophysics, etc., are determined. Properties of these species can exhibit large many-body and relativistic effects. Is study uses newly developed relativistic configuration interaction (RCI) algorithms, which are now as robust as the nonrelativistic equivalents. New algorithm improvements include parallelization of the RCI code and a two order of magnitude speed-up of the oscillator strength program. Hyperfine structure (hfs) calculations on the complicated $\mathrm{Nb}$ II $(\mathrm{d}+\mathrm{s}) * * 4$ levels were completed. By thoroughly including core-valence effects, average energy errors were reduced to $450 \mathrm{~cm}^{-1}$, and the magnetic dipole constant determined to $4 \%$. Hyperfine structure results were obtained for La II and U I in good agreement with experiment. A study of the lifetimes of astrophysically important $\mathrm{Nb}$ II $4 \mathrm{~d} 35 \mathrm{p}$ levels is nearly complete. Lifetimes will become one of the properties emphasized in this project.

\section{University of Nebraska at Lincoln Lincoln, NE 68588}

\section{Department of Physics and Astronomy}

\section{Dynamics of Collision Processes Starace, A.F. 402-472-2795}

This project seeks to obtain a unified point of view for understanding the dynamics of a number of collision processes involving electron-alkali systems, specifically, single- and multiphoton detachment of negative alkali ions, low-energy electron alkali scattering, and collisional detachment of negative alkali ions. Emphasis is placed on discovering propensity rules and characteristics of highly excited, correlated-electron states produced in these processes. Eigenchannel R-matrix methods will be used to perform the numerical calculations proposed. Recent work has focused on highly excited, correlated two-electron features in $\mathrm{Li}^{-}$and $\mathrm{Na}^{-}$hotodetachment processes. Calculated predictions have been compared and contrasted with similar calculated results for the negative hydrogen ion. 


\section{University of Nevada at Reno Reno, NV 89557}

\section{Department of Physics}

\section{Screening Resonances in Plasmas Winkler, $P$. 702-784-6792}

The interaction potential between those atomic ions and electrons in plasmas is being formulated. The force between the charges is modified by short-range order and dynamical screening effects introduced by neighboring ions and fast electrons, respectively. These modifications determine one-electron properties (e.g. spectral lines which serve as key quantities in plasma diagnostics) as well as more-electron properties (e.g. dielectronic recombination rates which account for plasma losses). The usual Debye-Hückel treatment of screening to encompass also dynamic screening effects is being extended to take into account ion motions, using a highly flexible and easily calculable algorithm. This algorithm allows evaluating simultaneously level broadening and continuum threshold lowering. The crucial features of line broadening and line splitting (due to deviations of the immediate ion environment from sphericity) have been incorporated into the theory. For the first time, an analytical formula for the broadening of transition lines has been found in the important case of a hydrogen plasma. An extension of the conventional framework of many-body theory has been made in the development of a multichannel propagator approach. The newly established computer code allows calculating atomic properties and processes in various plasma environments with an accuracy that compares well with the best atomic computations these days. The results of the first pilot study of energies and widths of scattering resonances have been published. The main focus of this study was the stability of the negative hydrogen ion in plasma environments, this ion being the main contributor to the opacity of white dwarf stars and one of the main contributors to the opacity of the solar chromosphere. The study supports a surprisingly high degree of stability of the negative hydrogen ion in low temperature and low density plasmas.

\section{University of New Mexico Albuquerque, NM 87131}

\section{Department of Physics and Astronomy}

\section{8. $H^{-}$Spectroscopy Bryant, H.C. 505-277-3044}

\section{$\$ 130,000$}

Experimental studies are being continued on the spectroscopy of the negative ion of hydrogen. Earlier work on this project has been done on an $800 \mathrm{MeV} \mathrm{H}^{-}$beam at LAMPF using laser beams Doppler-shifted into the vacuum ultraviolet. The last measurement made before this beam line closed was a study of the interaction of the $\mathrm{H}$ with a series of thin foils and a determination of the branching ratios for the production of parabolic substates of the hydrogen atom as a function of foil thickness. The carbon foils used, ranging in thickness from 10 to $500 \mu \mathrm{g} /$ $\mathrm{cm}^{2}$, were considered to cause sub-femtosecond perturbations of the negative ion. This extensive data set is currently under analysis. An experiment to study excess photon detachment of electrons from $\mathrm{H}^{-}$using a $35 \mathrm{keV}$ beam at Los Alamos is being designed and built. In this experiment, a seeded YAG 1.06 pulsed laser beam is focussed on the $\mathrm{H}^{-}$beam and the energy spectrum of the detached electrons observed. A series of energy peaks, separated by the energy of a single light quantum, is expected to appear. An experiment to observe the region of the Feshbach and shape resonance in $\mathrm{H}^{-}$under high resolution with a tunable VUV laser beam developed at Los Alamos is planned. Design work is proceeding to conduct similar research using a $400 \mathrm{MeV} \mathrm{H}^{-}$beam at Fermilab; this will allow the continuation of the high energy work begun at LAMPF. Planning is also underway for a collaborative study of two-electron systems on the U8 beam line at the Advanced Light Source at Lawrence Berkeley Laboratory.

\section{City College of New York New York, NY 10031}

\section{Department of Physics}

\section{Molecular Structure and Collisional Dissociation \\ Becker, K.H. \\ 212-650-5613

Electron collision techniques are used to probe the structure and the collisionally induced break-up of various technologically important species. We have studied the electron-impact ionization and dissociative ionization of $\mathrm{SO}_{2}$, methane $\left(\mathrm{CH}_{4}\right)$, and the free radicals $\mathrm{CH}_{3}, \mathrm{CH}_{2}$, and $\mathrm{CH}$. Work on $\mathrm{BCl}_{3}$ and the $\mathrm{BCl}$ radical as well as work on the silane radicals $\mathrm{SiH}_{\mathrm{x}}(\mathrm{x}=1-3)$ is in progress. The work involving the $\mathrm{CH}_{\mathrm{x}}$ and $\mathrm{SiH}_{\mathrm{x}}$ free radicals is motivated (i) by data needs for the further development and quantitative interpretation of novel diagnostics techniques applied to low-temperature plasmas (e.g., threshold ionization mass spectrometry) and (ii) by more fundamental aspects (comparison with calculations, investigation of potential scaling laws, and data trends for targets of similar structure). Simultaneously, we have extended a semiempirical method of calculating electron-impact ionization cross sections of atoms to the systematic calculations of ionization cross sections along the hydrogen-, helium-, and lithiumlike isoelectronic sequence, to the multiple ionization of atoms and to the ionization of molecules free radicals and clusters.

\section{University of Notre Dame Notre Dame, IN 46556}

\section{Department of Physics}

\section{Rydberg States in Multiply-Charged Ions Livingston, A.E. 219-631-7554 \\ $\$ 78,009$}

Atomic properties of multiply-charged ions are investigated using excitation of energetic heavy-ion beams. Spectroscopy of excited atomic transitions is applied from the visible to the extreme ultraviolet wavelength regions 
to provide accurate atomic structure and transition rate data in selected highlyionized atoms. High resolution position-sensitive detection has been introduced for measurements in the extreme ultraviolet region. The detailed structures of Rydberg states in highly charged berylliumlike ions are measured as a test of long-range electron-ion interactions. The measurements are supported by multiconfiguration Dirac-Fock calculations and by many-body perturbation theory, Additional studies of high-angularmomentum Rydberg transitions are being performed to establish reference wavelengths and improve the accuracy of ionization energies in highly charged systems. Precision wavelength measurements in highly charged few-electron ions are underway as tests of the most accurate relativistic atomic structure calculations. Precision lifetime measurements in laser-excited alkali atoms have been initiated to establish the accuracy of relativistic atomic many-body theory in many-electron systems:

\section{Pennsylvania State University, Lehman Lehman, PA 18627}

\section{Department of Physics}

\section{Electron Transfer, Ionization, and Excitation in Atomic Collisions Winter, T,G.; Alston, S,G. 717-675-9221}

Electron transfer, ionization, and excitation in intermediate- and high-energy ion-atom collisions are studied using theory, Recently, proton-sodium-atom collisions have been treated for proton energies ranging from 1 to 100 kiloelectronvolts. A Sturmian-pseudostate approach has been employed to account for the strong coupling among electron-transfer, ionization, and excitation channels, with generally very good agreement with experimental results. Several perturbative calculations (valid when transition probabilities are small) have also been carried out recently. A distorted-strong-potential-Born approach has been applied to electron transfer by protons from targets along isoelectronic sequences (for example, for the $1 \mathrm{~s}^{2} 2 \mathrm{~s}^{2}$ configuration for Be through $\mathrm{Ne}^{6+}$ targets) as well as sequences involving various stages of ionization (for example, Ne through $\mathrm{Ne}^{9+}$ ), and scaling rules have been determined. A second-order Faddeev approach has been applied to electron transfer in proton-helium-atom collisions at lower intermediate collision energies; there is good agreement with experimental differential cross sections at a proton energy of 293 kiloelectronvolts. Other perturbative studies of electron transfer have also been carried out.

\section{Rice University Houston, TX 77005}

\section{Department of Space Physics and Astronomy}

\section{Quantum Properties of Strongly Magnetized Plasmas \\ Weisheit, J.C, 713-527-4654}

The goal of this research program is to study effects of intense magnetic fields on atomic quantities, such as electronic structure, transition rates, and lineshapes, that are needed to determine quantum transport coefficients and equations of state. Strong fields (B $>10^{7}$ Gauss) arise in a variety of transient laboratory environments, z-pinches, many laser-produced plasmas, and even some chemical explosions as well as certain kinds of stars. In earlier work, the point of departure was Kadomtsev's generalization of the Thomas-Fermi equation for the atomic charge density $n(r)$. Correct axial symmetry was imposed at the onset. Numerical results for helium and carbon atoms in megagauss to gigagauss fields revealed that deformed atoms are much more tightly bound than those described by Kadomtsev's spherical model. Recent work has focused on the influence of aspherical charge densities on electrical resistivity in dense, strongly magnetized plasmas. The Boltzmann transport equation is being solved via Krook's approximation, which permits an effective collision frequency to be introduced for electron-ion scatterings.

\section{University of Southern California Los Angeles, CA 90089}

\section{Department of Physics and Astronomy}

\section{Amplitude Modulation of Atomic Wavefunctions \\ Cooke, W.E.}

213-740-1128

This project will use a novel laser excitation method in conjunction with the electron-electron interaction in a multi-electron atom to modulate the electronic wavefunction of a single electron within an excited atom. Using this method, many bits of digital information can be stored within a single atom. This project will provide experimental confirmation of this process. Methods will be investigated to retrieve the stored information, and the limits of the information storage capacity will be explored. These novel wavefunctions are expected to have significant effects on other radiative processes, which should be important for the development of new coherent radiation sources. These radiative effects will be examined. Current progress includes the development and characterization of a high-power, short-pulse blue laser and the construction of an interferometer for encoding time information onto the laser. Initial, low-power experiments have shown that the atom recognizes the laser pulses' timing. Future experiments will explore the high power limitations of this method. 


\section{Behavior of Atoms in a Strong Radiation Field \\ Shakeshaft, $R$. \\ 213-740-7888 \\ $\$ 104,000$}

This project is a theoretical study of the interaction of an atomic system with a strong (synchrotron or laser) radiation field. Atomic systems containing two electrons, e.g., helium and the negative hydrogen ion, are of primary interest. Among the processes being explored, double ionization is of special interest. Double ionization is challenging not only because of the importance of the electron-electron correlation but also because the boundary conditions for two-electron escape are complicated. We have developed and successfully applied a method for calculating differential cross sections for double ionization by one photon, and we are currently extending these calculations to include absorption by more than one photon; our eventual goal is to treat the radiation field nonperturbatively.

\section{University of Tennessee at Knoxville Knoxville, TN 37996}

\section{Department of Physics and Astronomy}

\section{The Production and Destruction of Metastable Negative Ions \\ Pegg, D.J. 615-974-7831}

The structure of few-electron atomic negative ions and the manner in which they interact with electromagnetic radiation via the process of photodetachment are investigated. The primary motivation for the work is to gain an understanding of the role of correlated electron motion in atomic structure. The work also has a potential application in areas involving cold plasmas such as those used in the processing of materials. Thus far, the negative ions of the first six elements of the periodic table have been studied. A crossed laser-ion beam apparatus is used to make energy- and angle-resolved measurements of the detached photoelectrons. The spectra provide information on photoelectron kinetic energies, yields, and angular distributions. Electron affinities, asymmetry parameters and cross sections have been determined from the measurements. Recent experiments include the first investigation of the angular distribution of photoelectrons detached from the excited negative ion of carbon and the first observation of a Feshbach resonance in the cross section for the photodetachment of the negative ion of lithium. Structure in detached electron spectra arising from ion-atom collisions has been identified with the autodetaching decay of shape resonance states in negative lithium and boron ions.
University of Texas at Austin Austin, TX 78712

\section{Department of Physics}

\section{State-to-State Energy Transfer in Atomic Collisions \\ Keto, J.W. \\ 512-471-5029 \\ $\$ 121,000$}

Measurements of state-to-state energy transfer rates for $\mathrm{Xe}\left(5 \mathrm{~d}, 5 \mathrm{~d}^{\prime}\right)$ states in collisions with other rare gases are being pursued. These results are relevant to kinetic models of excimer lasers and the nuclear-pumped, infrared xenon laser. In many previous experiments, strong coupling to these states through avoided crossings with Xe $6 \mathrm{p}, 6 \mathrm{p}^{\prime}$, $7 p$ ) were suggested, but not confirmed, because the dynamics of Xe(nd) could only be indirectly observed. The 5d excited states fluoresce in the far infrared, where detectors are too slow for studies of dynamics. Experiments are now being attempted to observe the dynamics of states of $\mathrm{Xe}\left(5 \mathrm{~d}, 5 \mathrm{~d}^{\prime}\right)$ using two-color, pulse-probe laser-induced fluorescence. An example sequence is first to excite Xe 6p[1/ $2 \mathrm{~J}_{0}$ in a two photon transition from the ground state. Collisional population of $5 \mathrm{~d}[1 / 2]_{1}$ is monitored by excitation to $\mathrm{Xe} 7 \mathrm{p}[3 / 2]_{2}$ using a laser tuned to $1149.44 \mathrm{~nm}$. By measuring the fluorescence of $\mathrm{Xe} 7 \mathrm{p}[3 / 2]_{2}$ as a function of the delay between the lasers, the dynamics for population of $\mathrm{Xe}(5 \mathrm{~d})$ can be measured. The results will be compared with those predicted by recently developed theory.

\section{University of Toledo Toledo, OH 43606}

\section{Department of Physics and Astronomy}

\section{Semiempirical Studies of Atomic Structure \\ Curtis, L.J. 419-537-2341}

The goal of the project is to develop a precise, comprehensive, and reliable base of atomic structure data for complex atomic ions through the use of precision experimental measurements, sensitive semiempirical data systematizations, and specialized theoretical computations. The properties studied involve both primary data (wavelengths, frequency intervals, relative intensities, production and decay rates, etc.) and derived structural parameters (energy levels, ionization potentials, electric polarizabilities, lifetimes, transition probabilities, excitation functions, etc.). Measurements are made primarily through the use of fast ion beam excitation methods, which are combined with available data from laser- and tokamak-produced plasmas, astrophysical observations, and conventional light sources. Large blocks of data are systematized and parametrized along isoelectronic, homologous, isoionic, Rydberg, and yrast sequences and series. Examples of investigations conducted during the past year include: (1) extensions of lifetime measurements in $\mathrm{Au}$ II and $\mathrm{Hg}$ III in the Pt sequence to the homologous systems $\mathrm{Ag}$ II and $\mathrm{Cd}$ III in the Pd sequence; (2) precision determinations of the dipole polarizabilities of $\mathrm{Mg}^{\text {plus }}, \mathrm{Zn}^{\text {plus }}$, and $\mathrm{Ca}^{\text {plus }}$ based on lifetime measurements and the quantitative specification of oscillator 
strength cancellation effects; (3) new measurements of allowed and forbidden $\Delta n=1$ transition probabilities for ions in the Be and Ne sequences, which permit predictive extrapolations of these quantities to systems for which lifetimes are immeasurably short.

\section{Negative Ion-Atom Collisions Kvale, T.J. 419-537-2980}

This research project involves the measurement of cross sections of the prominent scattering processes in relatively simple negative ionatom collision systems. The primary motivation for this work is to provide increasingly stringent tests of our understanding the physics of ionatom collisions at intermediate energy. Studies of single- and double-electron detachment have been recently completed. The experiments in progress involve measuring ion energy-loss $\mathrm{H}^{-}+$noble gas collisions over the energy range from 5 to $100 \mathrm{KeV}$. These measurements will permit the determination of absolute cross sections for the direct excitation of discrete target states and/or target ionization processes in which $\mathrm{H}^{-}$survives the collision. In addition to target ionization total cross sections, ionization cross sections differential in energy-loss will also be determined. To date, there are no other measurements or theoretical calculations for these inelastic scattering processes in the intermediate energy region. Depending on scheduling, the experiments will involve atomic hydrogen targets in addition to the noble gas targets.

\section{Tulane University New Orleans, LA 70118}

\section{Department of Physics}

\section{Few Electron Transitions in Atomic Collisions \\ McGuire, J.H. \\ 504-865-5520}

Describing reactions involving more than one electron requires information about the dynamics of few-electron phenomena, i.e., information required to describe how relatively complex, many-body systems change. Theoretical techniques for calculating cross sections for multielectron processes have been developed, which may be used to probe mechanisms for the dynamics of multielectron effects. One method uses a Born expansion in the projectile interaction strength, $\mathrm{Z}_{\mathrm{p}}$, and incorporates all terms in the electron-electron interaction. The leading term in $Z_{p}$ is reduced to a closed analytic form. The second order term is expressed as a one-dimensional energy integral over a product of two off-energy-shell first-order amplitudes, and so on. This method has been used to calculate cross sections for double excitation of helium to the $n=2$ levels of helium by the impact of protons, ions, electrons, and antiprotons. Cross sections have been analyzed for ionization plus excitation by charged particle impact in helium. Many-body perturbation theory has been used to calculate cross sections for double and single ionization in helium by photon impact. Preliminary calculations for two electron transitions in collisions of charged particles with molecules have also been performed.
Vanderbilt University

Nashville, TN 37235

\section{Department of Computer Science}

\section{Theoretical Studies of Atomic Transitions Fischer, C.F. 615-322-2926 $\$ 147,000$}

Atomic structure calculations are performed to predict properties such as energy levels, binding energies, transition probabilities, lifetimes, hyperfine structure, isotope shifts, and photoionization cross-sections. Accurate computational procedures are devised so that properties can be predicted even when they cannot be obtained from experiment and also to assist in the identification of observed data. The method used is the multiconfiguration Hartree-Fock (MCHF) method, optionally corrected for relativistic effects in the Breit-Pauli approximation. Fully relativistic Dirac-Fock calculations also are performed using the GRASP code. Recent calculations have stressed the application of systematic procedures, which include an estimate of the uncertainty in a predicted result. Special emphasis is on transition probabilities of lighter atoms. Intercombination lines have been investigated in $N$ IV and N III in the Breit-Pauli approximation. Results were checked using GRASP and extended to the isoelectronic sequence. A new procedure has been developed for transition calculations using independently optimized wavefunctions. Also under development are new algorithms using spline basis methods. The bound-state methods have been combined with spline methods to permit accurate determination of resonances in negative ions such as negative boron.

\section{University of Virginia Charlottesville, VA 22901}

\section{Department of Physics}

\section{Studies of Autoionizing States Relevant to Dielectronic Recombination Gallagher, T.F. 804-924-6817 $\$ 117,000$}

This program is focused on two electron processes in excited atoms, of practical importance in dielectronic recombination as occurs in fusion plasmas and in shortwavelength lasers. The experimental approach employed involves multistep laser excitation of the two valence electrons in alkaline earth atoms to doubly excited states, which usually decay by autoionization. This laser excitation process is the inverse of dielectronic recombination and gives detailed information about both autoionization and dielectronic recombination rates. Measurements in the past year have shown for the first time the enhancing effect of small electric fields ( 1 volt $/ \mathrm{cm}$ ) on dielectronic recombination. More precisely, enhancement of dielectronic recombination from a continuum of finite bandwidth in a broad autoionizing state was observed in a well controlled experiment. The energies and autoionization rates of $\mathrm{Mg}$ 3 pnf $J=2$ and 4 states have been measured and found to agree with an independent electron model. These measurements imply that the autoionization rates of all $\mathrm{Mg}$ 
3 pn $\ell$ (where $\ell \geq 3$ ) states can be calculated with confidence. These measurements complete those planned for $\mathrm{Mg} 3 \mathrm{pn} \ell$ autoionization rates.

\section{Western Michigan University Kalamazoo, MI 49008}

\section{Department of Physics}

\section{Near Threshold Excitation and Ionization of Open-Shell Atoms \\ Berrah, N. 616-387-4955

The objective of this research is to probe fundamental processes of atomic structure and the dynamics associated with the interaction of synchrotron radiation with atoms. The research aims at investigating, in atomic innershells, many-body effects due to the electron-electron interaction. Near-threshold photoexcitation-photoionization experiments will be performed to study electron correlation satellites in rare gases first and open-shell atoms. Quantitative studies of such processes are expected to yield important information about interaction mechanisms, providing benchmarks for developing theoretical models. Current activities are focused on investigating electron correlations in rare gases, and in particular in He. Measurements of double to single ionization between 280 and $1210 \mathrm{eV}$ have been performed at the BESSY synchrotron facility. These measurements, when compared with the most recent calculations, show the importance of initialstate correlations and final-state correlations. We have recently made measurements at the Advanced light Source (ALS) in Berkeley, California on the autoionization of $\mathrm{Ar}$ and $\mathrm{Ne}$ and on the excitation and photoionization of $\mathrm{Kr}$.

\section{Correlated Charge-Changing Ion-Atom Collisions \\ Tanis, J.A. 616-387-4960 \\ $\$ 102,000$}

Experimental investigations of atomic interactions in collisions of few-electron ions with neutral gas targets or electrons are conducted. Interactions involving excitation, ionization, and charge transfer (capture) are studied. The primary emphasis of this work is the study of two-electron processes, and in particular, those collision processes for which the electron-electron interaction is important. This work centers around three general areas of investigation: (1) emission of electrons along the beam direction and traveling with the beam velocity, i.e., cusp-electron production, (2) double (or multiple) ionization of helium or other targets, and (3) resonant recombination involving either resonant transfer excitation (RTE) or dielectronic recombination (DR). Specific collision processes are isolated and identified, for the most part, using coincidence techniques in which projectile charge-changing events are associated with electrons, recoiling target ions, or X-rays. Measurements are conducted using accelerators at Western Michigan University, Indiana University, Lawrence Livermore Laboratory, Michigan State University, and the Institute of Nuclear Research, Debrecen, Hungary.

\section{The College of William and Mary Williamsburg, VA 23187}

\author{
Depàrtmènt of Physics
}
274. Negative Ion Detachment Cross Sections Champion, R.L.; Doverspike, L.D. $\quad \$ 112,000$ 804-221-3510

The current goal of the research project is to develop an understanding of the collisional dynamics for systems where negative and positive ions collide with atoms or molecules as well as well-characterized metallic or metaloxide surfaces. The studies will focus on gas phase two-body collisions and on collisionally induced desorption of electron electrons and ions from surfaces. The collision energies for each of these experiments range from a few electronvolts up to about $0.5 \mathrm{keV}$. Cross sections for collisional and associative electron detachment, charge transfer, and negative ion-molecule reactions will be determined for reactants, which will include atomic oxygen, chlorine, and fluorine as targets. Additional measurements are planned for the reactants $\mathrm{H}_{3}{ }^{+}+\mathrm{H}_{2}$ and their isotopic variants. Experiments to be performed with surfaces will concentrate upon secondary ion and electron emission, which results from the impact of low-energy $(<500 \mathrm{eV})$ atomic species. In particular, the role of sputtered negative ions, some of which apparently autodetach, is being examined as a principal source of secondary electron emission for most metal oxide surfaces.

\section{Chemical Energy}

\section{University of Arizona Tucson, AZ 85721}

\section{Department of Chemistry}

\section{A Model Approach to Hydrodenitrogenation Catalysis Wigley, D.E. 602-621-4118}

$\$ 87,800$

Available mechanistic information regarding metalmediated hydrodenitrogenation (HDN) catalysis is not extremely detailed. Studies continue of soluble model compounds that stimulate HDN substrate/catalyst interactions. The primary focus is on the six-membered heterocyclic compounds such pyridine and its derivatives, bound to mid- or high-oxidation state tantalum centers. These studies have uncovered the following observations: (1) The $\eta^{2}(N, C)$ binding mode renders a pyridine ligand susceptible to an apparent nucleophilic attack by $\mathrm{LiR}$ or $\mathrm{RMgCl}$ reagents and results in $\mathrm{C}-\mathrm{N}$ bond cleavage. (2) This C-N bond scission occurs unambiguously via an intramolecular attack of a migrating alkyl ligand; therefore, the same metal center is capable of activating the pyridine $\mathrm{C}-\mathrm{N}$ bond and delivering the alkyl reagent that induces its scission. (3) This bond cleavage process occurs with a buildup of negative charge on the migrating alkyl or aryl ligand, i.e., the ligand migrates as an electrophile. These results offer new insight in HDN related processes, including the manner by which nitrogen heterocycles may 
be further degraded after C-N bond cleavage. Furthermore, this information is especially relevant to reactions of pyridine, since pyridine HDN often provides only a minor fraction of $\mathrm{C}_{5}$ products.

\section{Boston College Chestnut Hill, MA 02167}

\section{Department of Chemistry}

\section{High-Temperature Chemistry of Aromatic Hydrocarbons \\ Scott, L.T. $\$ 105,400$ \\ $617-552-8024$}

This work focuses on the fundamental molecular processes involved in the rearrangements and interconversions of polycyclic aromatic hydrocarbons (PAHs) under conditions of thermal activation. PAH ring systems figure prominently in the molecular architecture of coal, but prior to this systematic program of study, little was known about the chemical transformations that PAHs undergo at high temperatures, such as those employed in the uncatalyzed gasification and liquefaction of coal. This year a study has been completed of $\left[2 \mathrm{a}-{ }^{13} \mathrm{C}\right]$ acenaphthylene that provides dramatic new evidence for the hydrogenshift/ring-contraction reaction in benzene rings at high temperatures. Parallel studies on $\left[9_{-}^{2} \mathrm{H}, 9\right.$ $\left.{ }^{13} \mathrm{C}\right]$ phenanthrene and $\left[1-{ }^{2} \mathrm{H}, 1^{13} \mathrm{C}\right]$ naphthalene have provided the first compelling evidence that ortho hydrogen atoms on an aromatic ring switch places with one another at high temperatures and, as predicted, do so faster than the underlying carbon skeleton becomes scrambled. A new dicyclopentaanthracene and all three of the previously unknown dicyclopentapyrenes have been synthesized by pyrolysis reactions. The long-range objectives of this research are to uncover all the principal reaction channels available to PAHs at high temperatures and to establish the factors that determine which channels will be followed in varying circumstances.

\section{California Institute of Technology Pasadena, CA 91125}

\section{Department of Chemistry}

\section{Synthetic and Mechanistic Investigations of Olefin Polymerization Catalyzed by Early Transition Metal Compounds Bercaw, J.E. 818-395-6577 \\ $\$ 148,000$}

The objectives of this research program are (1) to discover new types of chemical transformations between hydrocarbons and transition-metal compounds; (2) to investigate their mechanisms; and (3) to explore the possibilities of coupling these transformations with others to catalyze chemical reactions for the preparation of fuels, commodity chemicals, and polymeric materials. A recent focus is the catalytic polymerization of olefins. Ziegler-Natta catalysis is a well-established and commercially very important process; however, it is clear that new (and superior) polymers with different microstructures and new homo-block copolymers could be made from the same readily available monomers if sufficient control over the catalytic process could be achieved. Racemic yttrocene and scandocene derivatives with linked cyclopentadienyl ligands have been prepared. The alkyl and hydride derivatives function as well-defined, single component, isospecific alpha olefin polymerization catalysts. Synthetic strategies to more active single component, isospecific catalysts are being developed. Well defined, syndiospecific catalysts have recently been prepared. A ligand capable of affording only one enantiomer of a chiral catalyst has been synthesized, and the X-ray structure of a chiral yttrium hydride dimer has been recently completed. The absolute facial preferences for olefin insertion into $\mathrm{Y}-\mathrm{H}$ and $\mathrm{Y}-\mathrm{C}$ bonds is under investigation using NMR methods that utilize a chiral partially deuterated olefin recently developed in the California Institute of Technology labs.

\section{University of California, Davis Davis, CA 95616}

\section{Department of Chemical Engineering}

\section{Effects of Supports on Metal Complexes and Clusters: Structure and Catalysis Gates, B.C. 916-752-3953 \\ $\$ 97,000$.}

The research is a fundamental investigation of the effects of supports on the structure and catalytic properties of metal complexes and clusters. The metals are Rh and Ir. The supports are $\mathrm{MgO}$ and zeolite LTL. MgO is used as high-area powders and as crystallographically defined ultrathin layers on single-crystal Mo. Like MgO, the zeolite is basic, incorporating $\mathrm{K}^{+}$and $\mathrm{Ba}^{2+}$ exchange ions. With precursors such as $\operatorname{Tr}(\mathrm{CO})_{2}$ (acac)], metal subcarbonyls such as $\left[\operatorname{Ir}(\mathrm{CO})_{3}(\mathrm{OMg})_{3}\right]$ are formed (where the braces denote groups terminating the $\mathrm{MgO}$ ). With precursors such as $\left[\mathrm{HIr}_{4}(\mathrm{CO})_{11}\right]^{-}$and $\left[\mathrm{Ir}_{6}(\mathrm{CO})_{15}\right]^{2-}$, supported clusters such as $\mathrm{Ir}_{4}$ and $\mathrm{Ir}_{6}$ will be formed. The supported species will be characterized structurally with IR, EXAFS, and NMR spectroscopies, TPD, $\mathrm{H}_{2}$ chemisorption, and imaging methods. The samples will be tested as catalysts for ethylene hydroformylation toluene hydrogenation, and nbutane hydrogenolysis. The goals are to determine how the support structure and composition affect the structure of the well-defined supported metal complexes and clusters and their catalytic properties.

\section{University of California, Irvine Irvine, CA 92717}

\section{Department of Chemistry}

\section{Synthesis and Chemistry of Yttrium and Lanthanide Metal Complexes \\ Evans, W.J. 714-824-5174 \\ $\$ 112,899$}

The research objectives are to study how the lanthanides, a series of inexpensive metals available in the United States with have unique physical properties that make them valuable components in optical and magnetic devices, can be effectively incorporated into useful, energy-saving materials and how their special physical 
properties can be translated into reactivity that efficiently transforms substrates like $\mathrm{CO}$ and $\mathrm{CO}_{2}$ into more valuable chemical products. To accomplish these goals, an understanding of how ligands affect the attachment of substrates and other compounds to the lanthanides is needed as well as information on the reactivity of these metals attached to ligands that are compatible with practical applications. Recent studies of alkoxide-ligated yttrium and lanthanide complexes have shown that heterometallic bridging can be used, not only to combine the lanthanides with other metals, but also to provide access to coordination environments previously unavailable. For example, insoluble lanthanide compounds of the type $\mathrm{LnZ}_{3}$, where $\mathrm{Z}=\mathrm{Me}, \mathrm{OMe}, \mathrm{NMe}_{2}$, can be solubilized with trialkyl group compounds to make reactive species of the type $\mathrm{NdGa}_{3}\left(\mathrm{NMe}_{2}\right)_{3} \mathrm{Me}_{9}$ and $\mathrm{YAl}_{3} \mathrm{Me}_{12}$. The latter compound can incorporate additional amounts of aluminum in the form of $\mathrm{Al}_{2} \mathrm{Me}_{6}$ trapped in channels in the solid state.

\section{University of California, Riverside Riverside, CA 92521}

\section{Department of Chemistry}

\section{Study of the Surface Chemistry of Hydrocarbon Radicals and of Carbonium Ions on Metal Oxide Surfaces Zaera, $F$ 909-787-5498

In this project the surface reactions of saturated hydrocarbons on metal oxides are being studied by using a combination of surface-sensitive techniques. The main long-term goal is to obtain specific relations between the reactivity of the oxides and the structural details of their surfaces. Given that the main difficulty in pursuing this kind of research has proven to be the low probability for the initial dissociative absorption of the hydrocarbon molecules, that step will be bypassed by exposing oxide surfaces directly to species resembling those expected to form on the surface after activation, that is, to moieties in either radical or ionic states. The relevant metal oxide surfaces will be prepared either by cleaving oxide single crystals or by growing films in situ via the oxidation of metal surfaces under vacuum and will be characterized by using X-ray photoelectron spectroscopy (XPS), ion scattering (ISS), and low-energy electron diffraction (LEED) in order to determine the stoichiometry, oxidation state of the constituent elements, and degree of crystallinity of the solids. The chemistry of hydrocarbon moieties either in the form of radicals or as carbonium ions will later be studied on those oxide surfaces. Radicals will be prepared in one of two ways, either by thermal or photolytic excitation of suitable absorbed precursors (alkyl halides of azo compounds), or by direct pyrolytic decomposition of those precursors in the gas phase. The cationic species will also be made in the gas phase by ionizing hydrocarbon molecules, mass filtering the ions of interest, and decelerating them to low kinetic energies $(1-10 \mathrm{eV})$. The species formed on the surface will be characterized by combining results from experiments using reflection-absorption infrared (RAIRS) and static secondary ion mass (SSIMS) spectroscopies, and the charge transfer between the absorbed moieties and the oxides will be measured directly by using XPS. Finally, the chemistry of these systems will be studied by using thermal desorption techniques.
University of California, Santa Barbara Santa Barbara, CA 93106

\section{Department of Chemical and Nuclear Engineering}

\section{Alkane Activation and Reactivity on Iridium, Platinum, and Ruthenium Surfaces \\ Weinberg, W.H. 805-893-8528

In this project, the dynamics and kinetics of molecular trapping, trapping-mediated activation, and direct dissociation of methane, ethane, propane, i-butane, and cyclopropane on the $\operatorname{Ir}(110)-(1 \times 2), \operatorname{Ir}(111), \operatorname{Pt}(110)-(1 \times 2)$, and $R u(001)$ surfaces will be studied. Both supersonic molecular beam scattering and "bulb" (microreactor) measurements will be made with evaluation of the reaction rates by mass spectrometry, Auger electron spectroscopy, and X-ray photoelectron spectroscopy. The rate coefficients of the elementary surface reactions (dehydrogenation, carbon-carbon bond cleavage, and hydrogenation with coadsorbed atomic hydrogen) will be quantified spectroscopically. The (vibrational) spectroscopies to be used are Fourier transform reflectionabsorption IR and high-resolution electron energy loss spectroscopies. These results will provide a valuable quantification of both the reactivity and the selectivity of $\mathrm{C}-\mathrm{H}$ bond activation for both trapping-mediated and direct dissociative chemisorption as a function of electronic and geometric structure within the Group VIII transition metals. During the past year, the kinetic rate parameters, preexponential factors and activation energies, for selective C-H, C-D, and C-C bond cleavage have been quantified on the $\operatorname{Ir}(\mathrm{III})$ surface for the molecules: di-1, ${ }_{1-}{ }^{13} \mathrm{C}_{-} \mathrm{C}_{2} \mathrm{H}_{6}, \mathrm{C}_{2} \mathrm{D}_{6}, \mathrm{C}_{3} \mathrm{H}_{8}, \mathrm{CH}_{3} \mathrm{CD}_{2} \mathrm{CH}_{3}, \mathrm{C}_{3} \mathrm{D}_{8}, \mathrm{n}-\mathrm{C}_{4} \mathrm{H}_{10}$, i$\mathrm{C}_{4} \mathrm{H}_{10}$, i- $\left(\mathrm{CH}_{3}\right)_{3} \mathrm{CD}$, i- $\left(\mathrm{CD}_{3}\right)_{3} \mathrm{CH}$, and $\left(\mathrm{CH}_{3}\right)_{4} \mathrm{C}$. In addition, the activation of di-1, $1,{ }^{13} \mathrm{C}-\mathrm{C}_{2} \mathrm{H}_{6}$ at defect sites has been examined. It was found that $\mathrm{C}-\mathrm{H}$ bond activation, with activation energies between $10.4 \mathrm{kcal} / \mathrm{mol}$ for ethane and $11.4 \mathrm{kcal} / \mathrm{mol}$ for higher alkanes, is favored by 0.44 to 0.63 $\mathrm{kcal} / \mathrm{mol}$ over C-D bond activation for primary, secondary, and tertiary C-H (C-D) bonds. Selective activation of secondary and tertiary bonds is manifested by activation energies that are $300 \mathrm{cal} / \mathrm{mol}$ and $100 \mathrm{cal} / \mathrm{mol}$ less than for primary $\mathrm{C}-\mathrm{H}$ bonds. The activation energy of carboncarbon bond cleavage in $\mathrm{C}_{3} \mathrm{H}_{6}$ and $\left(\mathrm{CH}_{3}\right)_{2} \mathrm{CD}_{2}$ is $18.4 \mathrm{kcal} /$ mol, but it is 19.1 in $\mathrm{C}_{3} \mathrm{D}_{8}$. In $\mathrm{i}-\mathrm{C}_{4} \mathrm{H}_{10}$, and $\mathrm{i}-\left(\mathrm{CH}_{3}\right)_{3} \mathrm{CD}$, the activation energy of $\mathrm{C}-\mathrm{C}$ bond cleavage is 17.47 , while for $\mathrm{i}-\left(\mathrm{CD}_{3}\right)_{3} \mathrm{CH}$ it is $18.0 \mathrm{kcal} / \mathrm{mol}$. In neopentane, $\mathrm{C}-\mathrm{C}$ bond cleavage has a $16.1 \mathrm{kcal} / \mathrm{mol}$ barrier. The observed secondary kinetic isotope effect in C-C bond activation for these alkanes reflects the required bending deformation of the methyl groups to allow for the proper coordination of the surface Ir atom to the $\mathrm{C}-\mathrm{C}$ bond. 


\section{Department of Chemistry}

\section{Studies Relevant to the Catalytic Activation of Carbon Monoxides Ford, P.C. 805-893-2443}

This research is concerned with quantitative investigations of fundamental reactions relevant to the catalytic activation of carbon monoxide and other $\mathrm{C}_{1}$ compounds. New carbonylation catalysts heterogenized on polystyrene-based polymers have been developed and exploratory studies are being carried out to apply water gas shift catalysts to the destruction of persistent pollutants. Fast reaction techniques have been applied to prepare and to investigate reactive organometallic intermediates relevant to the activation of hydrocarbons toward carbonylation and to the formation of carbon-carbon bonds via the migratory insertion of $\mathrm{CO}$ into metal alkyl bonds. The goals are to delineate the fundamental principles of catalytic processes for the activation of $C_{1}$ compounds such as $\mathrm{CO}$ and of hydrocarbons and other substrates in order to establish guidelines for the design and engineering of new chemical systems for more efficient and environmentally friendly applications of energy and chemical feedstocks

\section{Carnegie-Mellon University Pittsburgh, PA 15213}

\section{Department of Chemical Engineering}

\section{3. $\mathrm{H}_{2} \mathrm{SO}_{4}$-Modified $\mathrm{ZrO}_{2}$ and $\mathrm{ZrO} \mathrm{O}_{2} / \mathrm{SiO}_{2}$ Aerogels as Solid Superacids \\ Ko, E.I. 412-268-3857 \\ $\$ 97,000$}

Prehydrolysis was used to improve the homogeneity of zirconia and silica in a 50-50 (mol\%) mixed oxide aerogel. Specifically, varying the molar ratio of water to silicon precursor (the prehydrolysis ratio) allowed a fine degree of control of the product's textural and acidic properties. As prehydrolysis ratio was increased from 0.00 to $3.22 \mathrm{mols}$ $\mathrm{H}_{2} \mathrm{O} / \mathrm{mol} \mathrm{Si}$, molecular-scale mixing of the two oxides improved, resulting in increased acid site density, fractional Brönsted acidity, and 1-butene isomerization activity. The usefulness of prehydrolysis in promoting homogeneity of mixed oxides was further demonstrated for titania-silica aerogels over the entire composition range at a fixed prehydrolysis ratio. Samples prepared without prehydrolysis were found to have lower 1-butene isomerization activity, total site densities, and fractional Brönsted site populations than those prepared with prehydrolysis. These observations were explained in terms of the segregation of silica in non-prehydrolyzed samples in the form of silicarich patches that obscured much of an underlying titania-enriched core. The results of zirconia-silica and titania-silica together have clearly demonstrated the advantages of using sol-gel preparation to understand the acidic properties of mixed oxides.

\section{Colorado State University Fort Collins, CO 80523}

\author{
Department of Chemistry
}

\section{Polyoxoanion-Mediated Methane Activation and Functionalization: Molecular Design of New Homogeneous and New Solid-State/Heterogeneous Catalysts \\ Finke, R.G. \\ 970-491-2541 \\ $\$ 140,068$ \\ (14 months)}

A primary goal of this research is to develop a chemical paradigm for polyoxoanion supported transition-metal catalysis, with an emphasis on small molecule $\left(\mathrm{H}_{2}, \mathrm{O}_{2}\right.$, $\mathrm{H}_{2} \mathrm{O}_{2}, \mathrm{CH}_{4}$ ) catalytic activation and functionalization $\left(\mathrm{CH}_{4}, \mathrm{RH}\right)$. Again this past year, several projects were completed and the results published, specifically: (i) a paper detailing the discovery of novel polyoxoanion and $\mathrm{Bu}_{4} \mathrm{~N}^{+}$stabilized $\operatorname{Ir}(\mathrm{O})_{\sim 300}$ and $\operatorname{Ir}(\mathrm{O})_{\sim 900}$ nanoclusters; (ii) an important mechanistic paper detailing a more general approach to distinguishing heterogeneous from homogeneous catalysts (a long-standing problem in catalysis); (iii) a full paper detailing the synthesis and characterization of our prototype polyoxoanion-supported transition metal precatalyst, [(1,5-COD)Ir $\left.\bullet \mathrm{P}_{2} \mathrm{~W}_{15} \mathrm{Nb}_{3} \mathrm{O}_{62}\right]^{8-}$; and (iv) a full paper on the polyoxoanion-supported complex $\left[\left(\mathrm{C}_{5} \mathrm{Me}_{5}\right) \mathrm{Rh} \bullet \mathrm{P}_{2} \mathrm{~W}_{15} \mathrm{Nb}_{3} \mathrm{O}_{62}\right]^{7-}$. The discovery of reproducible size and reproducible activity $\mathrm{Ir}_{\text {approximately } 300}$ and $\operatorname{Ir}_{\text {\{approximately90\} }}$ nanocluster catalysts that can be isolated and redissolved, plus the high catalytic reactivity demonstrated for these novel materials, offer many possibilities for catalysis. These new materials are, therefore, the focus of present efforts.

\section{Diosmacycloalkanes as Models for the Formation of Hydrocarbons from Surface Methylenes \\ Norton, J.R. \\ 303-491-5609

While olefin exchange with diosmacyclobutanes occurs stereospecifically through a ring-opened intermediate, isomerization of dimethylmaleate is observed with the closely - related $\mathrm{Os}_{2}(\mathrm{CO})_{5}\left(\mathrm{Me}_{2} \mathrm{PC}\left(\mathrm{H}_{2}\right) \mathrm{PMe}_{2}\right)_{2}$. We are investigating the source of this difference in exchange mechanism, as well as attempting the synthesis of related compounds [such as $\mathrm{Os}_{2}(\mathrm{CO})_{7}\left(\mathrm{Me}_{2} \mathrm{PC}\left(\mathrm{H}_{2}\right) \mathrm{PMe}_{2}\right)$ ] to test the generality of the ring-opening mechanism in olefin exchange reactions. Protonation of mono- and dinuclear dialkyl and olefin osmium complexes gives cationic alkyl, alkylidene, and olefin complexes. In the case of $\mathrm{Os}(\mathrm{CO})_{4}\left(\mathrm{C}_{2} \mathrm{H}_{4}\right)$, both the kinetic and thermodynamic sites of protonation are $\mathrm{Os}$, but $\mathrm{C}_{2} \mathrm{H}_{4}$ inserts into the Os-H bond in the presence of as weak a coordinating ligand as $\mathrm{Et}_{2} \mathrm{O}$. We are examining the differences in reactivity of this variety of cationic Os complexes toward olefins, alkynes, and CO. Diosmacyclobutanes will be prepared with cyclic, strained olefins such as norbornene, cyclobutene, and cyclopropene to assess (1) the effect of ring strain on the relative binding affinities and (2) the potential for strained diosmacyclobutanes to cleave $\mathrm{C}-\mathrm{C}$ and Os-Os bonds to form tethered alkylidene complexes: $(\mathrm{CO})_{4} \mathrm{Os}=\mathrm{CHR}-\mathrm{RHC}=\mathrm{Os}(\mathrm{CO})_{4}$. 


\section{University of Colorado Boulder, CO 80309}

\section{Department of Chemistry and Biochemistry}

\section{Syntheses and Reactions of Pyrrole Complexes \\ DuBois, M.R. \\ 303-492-5039}

$\$ 98,838$

The objectives of the project are (1) to synthesize new transition metal pyrrole complexes and (2) to investigate how the metal ion coordination affects the reactivity of the heterocycle. An understanding of how coordinated pyrrole ligands might be activated toward reduction, ring opening, or nucleophilic addition reactions may provide a basis for understanding basic mechanisms of the hydrodenitrogenation catalysts. A series of indole complexes of the formula $\left[\left(\eta^{6}\right.\right.$-indole $) \operatorname{Ru}\left(\eta^{6}\right.$-cymene $\left.)\right](\mathrm{OTf})_{2}, 1$, have been synthesized and characterized. The acidities of the coordinated indole ligands in derivatives of 1 were quantitatively measured by $\mathrm{pH}$ titrations of the complexes in aqueous solution. The pKa value determined for coordinated indole was found to be 7.71. The deprotonated [(cymene)Ru(indolyl)]OTf derivatives, 2 , were also isolated and characterized by spectral methods. The data, particularly the ${ }^{13} \mathrm{C}$ data, suggested that the indolyl ligand maintains an $\eta^{6}$ coordination through the carbocyclic ring. This has been confirmed by an X-ray diffraction study of the 2,3dimethylindolyl complex. Reactions of 2 with electrophilic metal centers were carried out, and new heteronuclear complexes [(cymene)Ru(indolyl-N)Pd( $\left.\left.\mathrm{PPh}_{3}\right) \mathrm{Cl}_{2}\right] \mathrm{OTf}, \quad 3$, and [(cymene)Ru-(indolyl-N] $\left.]_{2} \mathrm{Cu}\right](\mathrm{OTf})_{3}, 4$, have been isolated and characterized. Further reaction of 4 with $\mathrm{Al}(\mathrm{OtBu})_{3} \mathrm{H}^{1}$ at $-78^{\circ}$ proceeded selectively to give a single hydride addition product. One-and two dimensional NMR data indicated that hydride addition to the 4 position of each carbocyclic ring had occurred. The $\mathrm{Cu}(\mathrm{I})$ coordination greatly enhanced the regioselectivity of this reaction relative to the nucleophilic additions to [(cymene)Ru(indolyl)]OTf.

\section{Columbia University New York, NY 10027}

\section{Department of Chemistry}

\section{Model Studies in Hydrocarbon Oxidation Parkin, $G$. 212-854-8247 $\$ 93,657$}

The research performed during the last grant period has continued with an investigation of the chemistry of molecular terminal chalcogenido complexes. By studying the chemistry of a series of complexes with $M=O, M=S, M=S e$, and $M=T e$ bonds, it is hoped that this research will provide results that are relevant to systems concerned with both hydrocarbon oxidation and hydrodesulfurization processes. For example, we have synthesized the first series of oxo, sulfido, selenido, and tellurido derivatives of hafnium $\mathrm{Cp}{ }_{2} \mathrm{Hf}(\mathrm{E})\left(\mathrm{NC}_{5} \mathrm{H}_{5}\right)(\mathrm{E}=\mathrm{O}, \mathrm{S}, \mathrm{Se}, \mathrm{Te})$, and the first mononuclear telluroformaldehyde complex of tantalum $\mathrm{Cp}^{*}{ }_{2} \mathrm{Ta}\left(\eta^{2}-\mathrm{TeCH}_{2}\right) \mathrm{H}$. Studies on these complexes have revealed interesting differences in the chemistry of the systems as a function of the chalcogen. For example, coupling and cleavage reactions play an active role in a variety of important transformations. However, in spite of the potential importance of reactions involving the interconversion of $[\mathrm{M}](\mathrm{E})_{2}$ and $[\mathrm{M}]\left(\eta_{2}-\mathrm{E}_{2}\right.$ moieties, relatively few well-characterized examples of such transformations have been described. Significantly, we have reported the first examples of such transformations for tellurium, thereby suggesting that such reactions are more facile for tellurium than its lighter congeners.

\section{University of Connecticut Storrs, CT 06269}

\section{Department of Chemistry}

\section{Synthetic Todorokite: Preparation, Characterization, and Applications Suib, S.L. 203-486-2797

The goals of this project are to prepare new octahedral molecular sieve (OMS) and octahedral layer (OL) materials by several methods including sol-gel, reflux, and autoclave methods; to prepare and characterize isomorphously substituted $\left(\mathrm{Ni}^{2+}, \mathrm{Cr}^{3+}\right.$ ) OMS and OL materials; to explore presently unused characterization methods for OMS and OL systems such as diffuse reflectance UVvisible spectroscopy; and to optimize catalytic properties of OMS and OL for dehydrogenation of alkanes to terminal olefins and oxidation of alkanes to terminal alcohols. Materials with transition metals substituted into framework or tunnel sites of OMS and OL will be prepared. Characterization of such systems will be done with a variety of methods in order to study structural, compositional, surface, electronic, electrical, morphological, thermal, magnetic, electron transfer, redox, and catalytic properties. In situ characterization methods using X-ray diffraction and absorption and optical (infrared) methods will be used to monitor changes that occur with OMS and OL catalysts during reaction. Specific compositions have been chosen in order to optimize catalytic properties in dehydrogenation and oxidation reactions of hexane and similar alkanes.

\section{University of Delaware Newark, DE 19716}

\section{Center for Catalytic Science and Technology}

\section{Synthetic Reactions of Oxametallacycles and Related Intermediates on Transition Metal Surfaces}

Barteau, M.A. 302-831-8905

The goal of this research is to understand, by means of surface science studies, the elementary processes involved in the synthesis of oxygen-containing fuels and chemicals on transition metals, and the dependence of these processes upon the nature of the catalyst. This research utilizes Temperature Programmed Desorption (TPD), High Resolution Electron Energy Loss Spectroscopy (HREELS), and X-ray Photoelectron Spectroscopy (XPS) 
as its principal tools. Application of these techniques has identified pathways, kinetics, and reaction intermediates relevant to the catalytic chemistry of oxygenates. Current research examines the chemistry of epoxides, halohydrins, and esters on transition metal surfaces in order to isolate and to characterize key oxametallacycle intermediates, to examine the regioselectivity of bond-making and bondbreaking processes of these oxygenates on metal surfaces, and to connect these observations to established and emerging examples in homogeneous and heterogeneous catalysis. Studies of ring opening and isomerization of light epoxides on rhodium and palladium single crystal surfaces have been completed, and the synthesis of epoxides on well-ordered silver surfaces is currently being investigated. This work holds the potential for establishing new principles for surface organic syntheses, of discovering new chemistry, and thus of providing guidance for the development of new catalysts and processes for oxygenate synthesis.

\section{Department of Chemistry and Biochemistry}

\section{Oxidation Catalysis with}

Tris(pyrazolyl)borate Metal Complexes
Theopold, K.H.
302-831-1546

This project involves the development of catalysts for the oxidation of organic substrates using dioxygen as the source of the oxygen. In particular, the approach involves coordination and symmetric cleavage of the $\mathrm{O}_{2}$ molecule into two reactive metal-oxo moieties by hindered tris(pyrazolyl)borate complexes of late transition metals. The feasibility of this scheme has been previously demonstrated using a set of cobalt complexes. In the initial phase of the research the mechanism of the cobalt mediated stoichiometric reaction will be elucidated in detail, and some reactions of the cobalt system $\left[\mathrm{Tp}^{\prime} \mathrm{Co}, \mathrm{Tp}^{\prime}=\right.$ hydridotris(3-t-butyl-5-methylpyrazolyl)borate] related to oxidation catalysis will be investigated. Building on this, the metal complexes will be modified to facilitate catalytic turnover. To this end the binding equilibrium for $\mathrm{O}_{2}$ must be shifted, and the ligands must be "hardened" against oxidative degradation. This will be done by appropriate substitution of the ligand and/or the metal. In the long term, catalytic oxidations of various substrates as well as the design of ligands for regio- and stereo-selective oxidations will be investigated.

\section{Harvard University Cambridge, MA 02138}

\section{Department of Chemistry}

\section{Mo-Catalyzed Heteroatom Removal Reactions: Effects of Promoters on Mechanism \\ Friend, C.M. 617-495-4052}

$\$ 103,000$

Desulfurization reactions have been investigated on Coand S-modified $\mathrm{Mo}(110)$ in an effort to understand promoters used in hydrodesulfurization catalysts. In parallel, the structures of several important intermediates have been determined using vibrational spectroscopy and near edge X-ray absorption fine structure. Sulfur is generally found to inhibit dehydrogenation reactions that lead to nonselective reaction, as specifically shown for 2,5dihydrothiophene and benzenethiol. Benzenethiol reacts via a disulfide intermediate, so that the total reactivity remains high. Investigations of Co overlayers indicate that Co promotes hydrogenolysis, leading to an increase in selectivity. The combined effect of $\mathrm{Co}$ and $\mathrm{S}$ on desulfurization chemistry are also currently under study.

\section{University of Illinois at Urbana-Champaign Uribana, IL 61801}

\section{School of Chemical Sciences}

\section{Electron Transfer Activation of Coordinated Thiophene Rauchfuss, T.B. 217-333-7355}

The presence of organic sulfur compounds in fossil fuels poses very serious environmental and engineering challenges. The most effective method for addressing these problems is through the hydrodesulfurization (HDS) process whereby the sulfur is removed by hydrogenolysis of C-S bonds in the fossil fuel matrix. The project objectives are threefold: (1) elucidate mechanisms for metal-catalyzed HDS, (2) develop new methods for desulfurization of fossil fuels, and (3) develop new uses for organosulfur components of fossil fuels. Most of these studies employ thiophenes as representative substrates. Experiments focus on HDS pathways that involve electron transfer to a metal-thiophene ensemble followed by protonation, i.e., heterolytic hydrogen activation. The stereochemistry and energetics for individual steps are examined for model systems based on ruthenium complexes. New desulfurization methods and new uses for the organosulfur components in fossil fuels are developed through the addition of nucleophiles to metal thiophene ensembles.

\section{Indiana University Bloomington, IN 47405}

\section{Department of Chemistry}

\section{Chemical Principles Relevant to Materials Precursor Design and Synthesis Caulton, K.G. 812-855-4798

The project objective is to determine, by a series of case studies, which chemical routes are particularly facile for the conversion of mixed-metal alkoxides, $\mathrm{M}_{\mathrm{a}} \mathrm{M}_{\mathrm{b}}^{\prime} \mathrm{L}_{\mathrm{n}}$, to solid-state materials $M_{a} M_{b}^{\prime} X_{m}$. The transformation from molecule to infinite lattice solid will be effected by thermolysis, hydrolysis, and plasma treatment. The groups $\mathrm{L}$ to be considered include simple hydrocarbon-derived alkoxides, heavily fluorinated alkoxides, and vicinal diolates. These are chosen to incorporate progressively more complex chemical features, each of whose typical reaction patterns are well-established. Chemically-facile routes are expected in certain cases because elimination of known 
neutral organic molecules can be envisioned. This research involves establishing whether such expectation will be realized under CVD processing conditions. Incorporation of groups $\mathrm{L}$ containing mobile protons $\left(\mathrm{H}^{+}\right.$will also be considered as a "trigger" for precursor processing at especially low temperatures. In every case, mechanistically diagnostic experiments will be executed in order to allow generalization of these results to make more rational the design of effective molecular precursors to technologicallyvaluable solid materials.

\section{Metal Alkoxides Models for Metal Oxides Chisholm, M.H. 812-855-6606 $\$ 122,900$}

Following on from recognition of the fact that alkoxidesupported clusters of molybdenum and tungsten have central $\mathrm{M}_{\mathrm{x}} \mathrm{O}_{\mathrm{y}}$ cores that mimic the structures of ternary metal oxides of those metals, an extensive organometallic chemistry associated with these species was discovered. These molecular species provide models for catalytic reactions involving these metal oxides. The molecular cluster originally formulated a $\mathrm{W}_{6}(\mu-\mathrm{H})_{4}\left(\mu-\mathrm{O}^{\mathrm{i}} \mathrm{Pr}\right)_{8}(\mathrm{H})\left(\mathrm{O}^{\mathrm{i}} \mathrm{Pr}\right)_{5}$, formed in the hydrogenation of $\mathrm{W}_{2}\left({ }^{\mathrm{i}} \mathrm{Bu}\right)_{2}\left(\mathrm{O}^{\mathrm{i}} \mathrm{Pr}\right)_{4}$, has been shown by a variety of labelling studies to be $\mathrm{W}_{6}(\mu-\mathrm{H})_{4}(\mu-$ $\left.\mathrm{O}^{\mathrm{i}} \mathrm{Pr}\right)_{7}\left(\mu-\mathrm{C}^{\mathrm{i}} \mathrm{Pr}\right)(\mathrm{H})\left(\mathrm{O}^{\mathrm{i}} \mathrm{Pr}\right)_{5}$ where the $\mu$-Cipr ligand arises from the degradation of an ${ }^{i} \mathrm{Bu}$ ligand by $\alpha-\mathrm{CH}$ activation. This cluster adopts a single geometry and shows extremely slow intramolecular exchange of $\mu$ and terminal ligands. By Nuclear Overhauser Effect difference spectroscopy an absolute assignment of the $5 \mathrm{H}$ ligands with their ${ }^{1} \mathrm{H}$ NMR signals has been possible along with several of the $\mathrm{O}^{\mathrm{i}} \mathrm{Pr}$ ligands. With this knowledge it has been possible to interrogate the reactivity of the cluster and show that it undergoes site selective $\mathrm{D}$ for $\mathrm{H}$ or $\mathrm{O}^{\mathrm{i}} \mathrm{Pr}-\mathrm{d}_{7}$ for $\mathrm{O}^{\mathrm{i}} \mathrm{Pr}$ exchange on reaction with $\mathrm{D}_{2}$ and $\mathrm{Pr}^{\mathrm{i}}-\mathrm{d}_{7} \mathrm{OD}$, respectively. The cluster also catalyses a slow hydrogenation of ethene to ethane by exclusive use of the terminal W-H ligand. $\mathrm{W}_{2}(\mu-\mathrm{H})(\mathrm{OR})_{7}$ compounds where $\mathrm{R}={ }^{\mathrm{i}} \mathrm{Pr}$ or ${ }^{\mathrm{c}}$ Pen react with $\operatorname{ArN}=\mathrm{NAr}$ compounds by way of reductive cleavage of the N-N bond. Related reactions with RNHNHR and $\mathrm{R}_{2} \mathrm{NNH}_{2}$ compounds have been investigated leading either to $\mathrm{RN}^{2-}$ or $\mathrm{R}_{2} \mathrm{NN}^{2-}$ containing compounds. A novel cluster $\mathrm{W}_{3}(\mu-\mathrm{H})\left(\mathrm{O}^{\mathrm{i}} \mathrm{Pr}\right)_{9}(\mathrm{NPh})(\mathrm{py})$ has been characterized. $\mathrm{W}_{2}\left(\mathrm{OCH}_{2}{ }^{\mathrm{t}} \mathrm{Bu}\right)_{6}(\mathrm{py})_{2}$ in hydrocarbon solutions has been shown to act as a selective catalyst for the hydrogenation of linear 1,3-dienes to 3-enes, cyclic conjugated dienes to cyclic monoenes. $\alpha$ - and internal olefins are not hydrogenated but the strained cyclic enes, norbornene and cyclopentene, are converted to norbornane and cyclopentane, respectively.

\section{A Model Approach to Vanadium Involvement in Crude Oil Refining Christou, $G$. 812-855-2399}

The project is directed toward identifying the initial fate of crude oil vanadyl impurities under the reducing and sulfur-rich conditions of industrial hydrodemetallation (HDM) and hydrodesulfurization processes. The impurities are ultimately converted to insoluble vanadium sulfides (primarily $\mathrm{V}_{2} \mathrm{~S}_{3}$ and $\mathrm{V}_{3} \mathrm{~S}_{4}$ ), which lower the activity of, and eventually poison, the Mo heterogeneous catalyst. Recent work has concentrated on characterization of discrete V/S clusters that represent models for intermediate stages of $\mathrm{V}$ sulfide polymer growth. The complex $\mathrm{V}_{2} \mathrm{O}(\mathrm{SPh})_{4}(\mathrm{bpy})_{2}\left(\mathrm{bpy}=2,2^{\prime}\right.$-bipyridine) contains a $\left[\mathrm{V}_{2}(\mu-\mathrm{O})(\mu-\mathrm{SPh})_{2}\right]^{2+}$ core and contains both a V-V single bond and antiferromagnetically coupled electrons. It has been studied by VT magnetic susceptibility, VT ${ }^{1} \mathrm{M}$ NMR studies, and EHT MO calculations. The related anion $\left[\mathrm{V}_{2}\left(\mathrm{SCH}_{2} \mathrm{CH}_{2} \mathrm{~S}\right)_{4}\right]^{2-}$ has been identified as also possessing a V-V bond and antiferromagnetically coupled electrons and has been studied similarly. In contrast, $\left[\mathrm{V}_{3} \mathrm{~S}\left(\mathrm{~S}_{2}\right)_{3}(\mathrm{bpy})_{3}\right]^{+}$is diamagnetic, the first V/S cluster to be so, and represents a model for the currently unidentified, diamagnetic V/S species that have been detected by ${ }^{51} \mathrm{~V}$ NMR on the surface of partially spent Mo catalysts. Other complexes under study include $\mathrm{V}_{3} \mathrm{O}_{2} \mathrm{Cl}_{2}\left(\mathrm{SCH}_{2} \mathrm{CH}_{2} \mathrm{~S}\right)_{2}(\mathrm{bpy})_{2}, \quad \mathrm{~V}_{3} \mathrm{OCl}_{3}\left(\mathrm{SCH}_{2} \mathrm{CH}_{2} \mathrm{~S}\right)_{2}(\mathrm{bpy})_{2}$, and $\left[\mathrm{V}_{6} \mathrm{O}_{2} \mathrm{~S}_{4}\left(\mathrm{SCH}_{2} \mathrm{CH}_{2} \mathrm{~S}\right)_{6}\right]$, which represent discrete models of vanadyl units adsorbed on the surface of the growing $\mathrm{V}_{2} \mathrm{~S}_{3} / \mathrm{V}_{3} \mathrm{~S}_{4}$ phases. In addition, the work has been extended to include a variety of $\mathrm{V}$ carboxylate complexes; the latter organic functionality is common in crude oils.

\section{University of Iowa Iowa City, IA 52242}

\section{Department of Chemistry}

\section{Synthesis and Chemistry of Cationic $d^{0}$ Metal Alkyl Complexes \\ Jordan, R.F. 319-335-2212}

The objective of this research is to design and synthesize new types of electrophilic organometallic complexes for use in fundamental studies of olefin polymerization and $\mathrm{C}-\mathrm{H}$ activation chemistry, and for exploitation in catalysis. Earlier studies of $\mathrm{Cp}_{\mathrm{p} 2} \mathrm{Zr}(\mathrm{R})(\mathrm{L})^{+}$complexes identified the key features required for high insertion reactivity in early metal systems: an electrophilic metal center, a $\mathrm{d}^{\mathrm{o}}$ metal electron configuration, and one or more vacant (or virtual) coordination sites cis to the M-R ligand. Current work is directed to the development of new classes of cationic early metal alkyls, which incorporate these features in non-C $\mathrm{p}_{2} \mathrm{M}$ ligand environments. A series of $\mathrm{Zr}$ and $\mathrm{Hf}$ alkyl complexes $\left(\mathrm{N}_{4}\right.$-macrocyle) $\mathrm{M}(\mathrm{R})_{2}\left(\mathrm{R}=\mathrm{CH}_{3}\right.$, $\mathrm{CH}_{2} \mathrm{Ph}, \mathrm{CH}_{2} \mathrm{SiMe}_{3}$ ) containing dianionic tetra-aza macrocycles $\left(\mathrm{N}_{4}\right.$-macrocycle $=\mathrm{Me}_{8}$-taa, $\mathrm{Me}_{4}$-taen $)$ in place of $\mathrm{Cp}$ ligands has been prepared. The pockets of these macrocycles are too small to accommodate the large group 4 metal ions, so the metal sits out of the $\mathrm{N}_{4}$ plane and cis structures are imposed. Base-stabilized cations $\left[\right.$ cis- $\left(\mathrm{N}_{4}\right.$-macrocycle $\left.) \mathrm{M}(\mathrm{R})(\mathrm{L})\right]\left[\mathrm{BPh}_{4}\right](\mathrm{L}=\mathrm{THF}$, $\left.\mathrm{RCN}, \mathrm{PMe}_{2} \mathrm{Ph}\right)$, and base-free cationic systems $\left[\left(\mathrm{N}_{4}\right.\right.$ macrocycle $) \mathrm{M}(\mathrm{R})]\left[\mathrm{B}\left(\mathrm{C}_{6} \mathrm{~F}_{5}\right)_{4}\right]$, have been prepared by protonolysis routes. The base-free systems are moderately active ethylene polymerization catalysts. One example, (Mes-taa) $\mathrm{Hf}\left(\mathrm{CH}_{3}\right)+$, also undergoes clean single insertion of vinyltrimethylsilane, and clean double insertion of dimethylacetylene. Ortho $\mathrm{C}-\mathrm{H}$ activation of 2-methylpyridine and vinyl $\mathrm{C}-\mathrm{H}$ activation of 2vinylpyridine have also been observed with these cationic systems. Cationic alkyls based on tetradentate Schiff base ligands, e.g., $\left(F_{6}\right.$-acen $) \mathrm{Zr}(\mathrm{R})_{+}$, have been prepared more recently. These systems are active olefin polymerization catalysts in the presence of $\mathrm{AIR}_{3}$ cocatalysts. Chiral analogues catalyze the stereoselective polymerization of propylene to isotactic polypropylene. Current efforts are 
focused on more highly electron-withdrawing chelating ligands, which should maximize the electrophilicity of the metal center in these systems and thus increase reactivity.

\section{Kansas State University Manhattan, KS 66506}

\section{Department of Chemistry}

\section{Homogeneous Models of Ammoxidation Catalysis \\ Maatta, $E$. \\ 913-532-6687

The researchers continue to exploit their discoveries of simple and efficient routes to a wide variety of organoimido-substituted derivatives of the hexamolybdate cluster, $\left[\mathrm{Mo}_{6} \mathrm{O}_{19}\right]^{2-}$. A homologous series of arylimidohexamolybdates of the formula $\left[\mathrm{Mo}_{6} \mathrm{O}_{(19-x)}(\mathrm{NAr}) x\right]^{2-}(\mathrm{Ar}=$ 2,6-diisopropylphenyl; $x=1,2,3,4,5$ ) has been prepared and comprehensively characterized by cyclic voltammetry, UV/VIS spectrophotometry, multinuclear $\left({ }^{1} \mathrm{H},{ }^{14} \mathrm{~N},{ }^{95} \mathrm{Mo}\right)$ NMR spectroscopy, and single-crystal X-ray diffraction studies. Using the sterically less-demanding $o$-tolylimido ligand $\left(\mathrm{NAr}^{\prime}\right)$, a polyimido-hexamolybdate species, $\left[\mathrm{MO}_{6} \mathrm{O}_{14}\left(\mathrm{NAr}^{\prime}\right)^{6}\right]^{2-}$, whose structure features the first incorporation of an organoimido ligand into a $\mu^{2-}$ bridging site within the hexamolybdate has been prepared. The reactivity of this unique imido ligand is being investigated. Reactions designed to prepare hexamolybdate derivatives bearing benzyl- and allylimido ligands have led instead to reduced hexamolybdate species and the production of small amounts of benzonitrile and acrylonitrile, respectively. Studies of these transformations are underway.

\section{Lehigh University Bethlehem, PA 18015}

\section{Department of Chemical Engineering}

\section{Molecular Structures and Reactivity of Mixed Metal Oxide Monolayer Catalysts Wachs, I.E. 215-758-4274 $\$ 95,551$}

Metal oxide monolayer catalysts, supported metal oxide catalysts possessing the active metal oxide components as a surface phase, find extensive applications in the energy industries of petroleum refining, pollution control from power generation plants, and automotive pollution control. To help bridge the knowledge gap between model and industrial metal oxide monolayer catalysts, a fundamental research program will address the relationships between the molecular structures and surface acidity and the molecular structures and surface redox chemistry of mixed metal oxide monolayer catalysts. For the fundamental surface acidity portion of the research program the alumina-supported tungsten oxide system will be the focus of the investigation, and for the fundamental surface redox chemistry portion of the research program the alumina-supported vanadium oxide system will be the focus. The influence of secondary metal oxides upon the molecular structures and reactivity of these systems will be investigated. The molecular structures will be primarily determined with in situ Raman spectroscopy, but complementary structural spectroscopies (solid state nuclear magnetic resonance (NMR) and extended X-ray absorption fine structure (EXAFS) and X-ray absorption near-edge structure (XANES)) will also be applied. The surface chemistry will be probed by surface acidity and surface redox measurements. This fundamental information should allow better understanding of the synergistic interactions that occur in mixed surface metal oxides.

\section{Department of Chemistry}

\section{Mechanisms and Controlling Characteristics of the Catalytic Oxidation of Methane}

Klier, K.; Simmons, G.W.;

$\$ 124,000$

Herman, R.G.

610-758-3577

The general objectives addressed in this research are: the mode of methane activation on metals; the structuresensitivity of the $\mathrm{C}-\mathrm{H}$ bond activation; the nature of surface species originating from methane, oxygen, and dopants; the relationship between surface structure and dynamics of elementary catalytic steps; and the controlling characteristics of partial oxidation of methane. Palladium was the metal of choice because of its ability to activate methane at relatively low temperatures and a weak Pd-O surface bond. New fundamental methodology involving angle-resolved X-ray photoelectron spectroscopy (ARXPS), surface core level shifts, and X-ray photoelectron diffraction (XPD) at high energy resolution and valence band (VB) spectroscopy has also been developed. The resultant model of angular dependence in the photoelectron intensity attenuation has been extended to other overlayer systems (i.e., CO, S, Cl, and NO on Pd[100]), as well as to extraction of information of the initial state atomic orbital character of trigonal prismatic layered $\mathrm{MoS}_{2}$.

\section{Louisiana State University Baton Rouge, LA 70803}

\section{Department of Chemical Engineering}

\section{Gallium Zeolites for Light Paraffin Aromatization \\ Price, G.L. 504-388-3068}

Gallium loaded MFI (which is useful for light paraffin aromatization) is currently being investigated, and some new areas of research closely related to the original work have been opened. Recent work has focused on the fate of protons when gallium or other metals are driven into the zeolite. Proton depletion can be detected in all cases and catalytic activity can be correlated with the decreasing proton and increasing metal cation content. IR investigations confirm the proton replacement on metal incorporation, and have also been used to detect restoration of proton acidity that occurs on oxidation or steaming of gallium-containing materials. New avenues of research include copper and indium loaded materials and the use of zeolite-beta as a substrate. 


\section{Department of Chemistry}

\section{Polynuclear Aromatic Hydrocarbons with Curved Surfaces: Models and Precursors for Fullerenes Rabideau, P.W. 504-388-8859

The remarkable discovery that buckminsterfullerene, $\mathrm{C}_{60}$, is a stable molecule has led to a flood of research focused on this new family of three-dimensional carbon cages known as fullerenes. These unique structures can be produced by laser vaporization of graphite or coal, and metal derivatives show promise as superconductors. This program deals with the synthesis, structural study, and chemistry of polynuclear hydrocarbons with carbon frameworks represented on the buckminsterfullerene surface. These curved-surface hydrocarbons are expected to. serve as models for the fullerenes in some of their chemical and physical properties. The simplest example of such a hydrocarbon is corannulene, $\mathrm{C}_{20} \mathrm{H}_{10}$, which represents the polar cap of buckminsterfullerene. However, corannulene undergoes rapid bowl-to-bowl inversion that compromises its properties as a curved-surface hydrocarbon, and this program produced the first "locked" bowl-shaped hydrocarbon by the addition of a second five-membered ring to corannulene producing cyclopentacorannulene. More recently, this program produced the first semibuckminsterfullerene $\left(\mathrm{C}_{30} \mathrm{H}_{12}\right)$ or "buckybowl" representing half of the $\mathrm{C}_{60}$ surface. A second "buckybowl" has now been produced that corresponds to exactly half of buckminsterfullerene. In theory, this $\mathrm{C}_{30} \mathrm{H}_{12}$ might be dimerized to ultimately produce buckminsterfullerene itself, and this exciting reaction is being explored.

\section{University of Louisville Louisville, KY 40292}

\section{Department of Chemistry}

\section{Metallocarboxylate Chemistry Gibson, D.H. 502-852-5977}

This project is concentrated on the organometallic chemistry of $\mathrm{CO}_{2}$. In particular, focus is on the synthesis, characterization, and reactions of bimetallic compounds with carbon dioxide bridging ligands. The compounds are models for intermediates in the catalytic fixation of $\mathrm{CO}_{2}$. In the present period systems have been studied that have the carboxyl carbon bound to a late transition metal ( $\mathrm{Fe}$ or $\mathrm{Re}$ ) and one or both carboxyl oxygens bound to an $\mathrm{SnR}_{3}$ moiety ( $\mathrm{R}=\mathrm{Me}, \mathrm{n}-\mathrm{Bu}$, or $\mathrm{Ph}$ ). The thermolysis reactions of ten such compounds, six of which have been characterized by X-ray crystallography, have been examined in an effort to correlate structural parameters with the propensity for decarboxylation or disproportionation. Factors that influence the ease and mode of decomposition are: the presence of electron-donating or electron-withdrawing groups on either metal center and whether one or both oxygens are coordinated to the tin atom. Most important, however, in determining whether simple decarboxylation occurs or disproportionation results is the stability of the corresponding transition metal anion. In cases where this anion is known to be stable, decarboxylation takes place;otherwise, disproportionation predominates.
Also, research has been initiated on bimetallic compounds having both late and early transition metals bound to bridging $\mathrm{CO}_{2} ; \mathrm{Cp}{ }^{*} \mathrm{Ru}\left(\mathrm{CO}_{2}\left(\mathrm{CO}_{2}\right) \mathrm{Zr}(\mathrm{Cl}) \mathrm{Cp}_{2}\right.$ and $\mathrm{Cp}^{*} \operatorname{Re}(\mathrm{CO})(\mathrm{NO})\left(\mathrm{CO}_{2}\right) \operatorname{Zr}(\mathrm{Cl}) \mathrm{Cp}_{2}$ have been made and structurally characterized.

\section{University of Maryland at College Park College Park, IMD 20742}

\section{Department of Chemistry and Biochemistry}

\section{Odd-Electron Organometallic Chemistry of Relevance to Hydrocarbon Functionalization}

Poli, $R$. 301-405-1809

Significant progress has been achieved in the investigation of transition metal hydride complexes that are potential precursors to highly unsaturated odd-electron organometallics. High yield syntheses of compounds $\mathrm{Cp}^{*} \mathrm{MoH}_{3}$ (dmpe) and $\mathrm{Cp}^{*} \mathrm{MoH}_{3}$ (dppe) have been developed. The electrochemical oxidation of these materials affords relatively long-lived, EPR active radicals whose mechanism of decomposition is currently being investigated. Such a mechanism may involve either deprotonation or solvent-assisted disproportionation. To elucidate this mechanism, the protonation of the above materials is also being investigated. Complexes $\left[\mathrm{Cp}^{*} \mathrm{MoH}_{4}(\mathrm{~L}-\mathrm{L})\right]^{+}$and $\left.\left[\mathrm{Cp}^{*} \mathrm{MoH} \mathrm{H}_{2}(\mathrm{MeCN}) \mathrm{L}-\mathrm{L}\right)\right]^{+}$have been generated and their solution structure is being investigated by NMR techniques. The products of oxidation and protonation of $\mathrm{Cp}$ * $\mathrm{MoH}_{5}\left(\mathrm{PMe}_{3}\right)$ are also being investigated. Compound $\mathrm{CpMoH}\left(\mathrm{PMe}_{3}\right)_{3}$ also gives a relatively stable radical upon one-electron oxidation. Protonation takes place faster at the hydride ligand to give an intermediate complex with a labile $\mathrm{H}_{2}$ ligand which is immediately replaced if the reaction is carried out in $\mathrm{MeCN}$, to give $\left[\mathrm{Cp}^{*} \mathrm{Mo}(\mathrm{MeCN})\left(\mathrm{PMe}_{3}\right)_{3}\right]^{+}$(protonation at the metal) which is obtained when the reaction is carried out in a non-coordinating solvent. This classical dihydride does not eliminate $\mathrm{H}_{2}$ in $\mathrm{MeCN}$ on a time scale of months at room temperature \{exclamation_point\} Hydrogen atom abstraction reactions by the trityl radical on the above hydride materials are also of current interest in the laboratory. The above trihydrides react with $\mathrm{Ph}_{3} \mathrm{C}$ in a $1: 3$ ratio, even if a substoichiometric amount of trityl: is used. The final products, which formally derive from the 15-electron " $\mathrm{Cp}^{*} \mathrm{Mo}(\mathrm{L}-\mathrm{L})$ " species, are currently under in. vestigation. Future work will be directed to the detailed mechanistic investigation of the $\mathrm{H}$ atom abstraction reactions on mono and polyhydride complexes and their follow-up chemistry, with a particular interest in intraand inter-molecular $\mathrm{C}-\mathrm{H}$ activation processes. 
Massachusetts Institute of Technology Cambridge, MA 02139

\section{Department of Chemistry}

\section{High-Pressure Heterogeneous Catalysis in a Low-Pressure, Ultrahigh Vacuum Environment \\ Ceyer, S.T. \\ 617-253-4537 \\ $\$ 109,000$}

The major thrust of this project is to carry out high-pressure, heterogeneous catalytic reactions in a lowpressure, ultrahigh vacuum environment. These studies have now become possible because of the culmination of several investigations in the laboratory over the last five years resulting in the development of new physical processes and techniques: collision-induced absorption; collision-induced recombinative desorption; bulk vibrational spectroscopy; and the synthesis of adsorbed, reactive intermediates by translational and collisioninduced activation. These new processes allow the simulation of a high-pressure environment while maintaining the single-collision conditions in which microscopic reaction steps and intermediates can be elucidated and detected by molecular beam scattering coupled with highresolution electron energy loss spectroscopy. Results to date show that bulk $\mathrm{H}$ is the reactive species in the high pressure reaction involving the hydrogenation of $\mathrm{C}_{2} \mathrm{H}_{4}$.

\section{Controlled Synthesis of Polyenes by Catalytic Methods \\ Schrock, R.R. 617-253-1596}

A way has been found to synthesize totally new polyenes in a controlled living fashion from dipropargyl derivatives employing well-characterized alkylidene complexes of the type $\mathrm{M}\left(\mathrm{CHCMe}_{2} \mathrm{R}\right)(\mathrm{NAr})\left(\mathrm{OR}^{\prime}\right)_{2}(\mathrm{M}=\mathrm{Mo}$ or $\mathrm{W}, \mathrm{R}=\mathrm{Me}$ or $\mathrm{Ph}, \mathrm{Ar}=2,6$ diisopropylphenyl, $\mathrm{R}^{\prime}=\mathrm{OCMe}_{3}, \mathrm{OCMe}_{2}\left(\mathrm{CF}_{3}\right)$, $\mathrm{OCMe}\left(\mathrm{CF}_{3}\right)_{2}$, or various phenoxides) as catalysts. Dipropargyl derivatives of the type $\mathrm{HC} \equiv \mathrm{CCH}_{2} \mathrm{XCH}_{2} \mathrm{C} \equiv \mathrm{CH}$ $\left(\mathrm{X}=\mathrm{NR}, \mathrm{O}, \mathrm{C}\left(\mathrm{CO}_{2} \mathrm{R}\right)_{2}\right.$, SiMe 2 , and so forth) are cyclopolymerized to give soluble polyenes that contain either six-membered rings (head-to-tail cyclopolymerization) or five-membered rings (tail-to-tail cyclopolymerization). The reaction can be controlled by varying the solvent and the type of catalyst so that "dangling"chains resulting from simple insertion of one of the propargyl groups are absent. Addition of one of the acetylene bonds to an alkylidene to yield a new disubstituted alkylidene normally would essentially terminate polymerization, since the disubstituted alkylidene would not react readily with more terminal acetylene. This problem is avoided by the speed of the intramolecular cyclization reaction to give a fivemembered ring and a new monosubstituted alkylidene. This new polymerization reaction will lead to a large number of new materials since the conditions of polymerization are relatively mild (versus Ziegler-Natta conditions) and many functionalities therefore tolerated. In addition to investigating the scope and details of this new controlled cyclopolymerization reaction, the properties (nonlinear, conductivity, electrochemical, and so forth) of these new materials as a function of chain length will be studied, a fundamental question that remains largely unresolved in the area of unsaturated polymers (polyanilines, polythiophenes, polyparaphenylene, and so forth). It seems possible that a wide variety of new materials will become available that may rival the more established unsaturated polymers in applications, as well as fundamental research, because of the control exercised. in their preparation. A catalyst has now been prepared that cyclopolymerizes dipropargyl diethylmalonate to only six-membered rings, and another that polymerizes otrimethylsilyphenylacetylene in a living manner to give low polydispersity polyenes that contain between 10 and 100 double bonds. Nonlinear optical measurements on both types of polymers are being carried out in order to correlate $\beta$ and $\gamma$ with chain length and structure.

\section{University of Massachusetts at Amberst Amherst, MA 01003.}

\section{Department of Chemical Engineering}

\section{Zeolite Characterization and Dynamics: The Effect on Molecular. Transport and Catalyst Selectivity \\ Conner, W.C.; Laurence, R.L.; Ragle, J:L. $\$ 160,048$ 413:545-0316 \\ (20 months)}

Zeolitic materials are most often crystalline aluminasilicates with microporosity (less than $20 \AA$ ) created by interconnected ring-like structures. These channels give sorbing molecules access to the intraparticle surface where chemisorption and reactions occur. Since the channels within the lattice are similar in size to sorbing molecules, the term "configurational diffusion" has been used to describe intraparticle transport(1). The limited size of the products for the reactions of hydrocarbons, selective sorption, and selectivity in isomerization and trans-alkylation reactions have been ascribed to this "shape selectivity." This research focuses on three related aspects of zeolites: the mutual interactions between adsorbing molecules and the zeolite lattice, the nature of the pore structure of the zeolite characterized during adsorption; and the influence of extreme steric constraints on cracking and isomerization reactions for cycloalkanes. Earliest perceptions of the pore structure within a zeolite have depended on the visualization of the $\mathrm{Si}(\mathrm{Al})$-oxygen crystalline bond network. This representation and analysis depends upon an image of a fixed pore configuration based primarily upon X-ray diffraction (XRD) studies of the solid structure. Recent studies employing solids nuclear magnetic resonance (NMR) and in situ XRD have documented that the shape of the adsorbing pores can change on adsorption. More recently, detailed spectroscopic studies of adsorption and of adsorbing molecules have begun to provide a picture of the pore structure and the sorbing species during sorption. In situ infrared spectrometry (specifically far-FTIR) and thermal or gravimetric analyses (DTA and TGA) can also be employed to understand the dynamic configurational changes in the sorbing species and the energetics of these interactions. Several of these techniques have been developed, and each will be used in concert to understand the effects of the interactions between adsorbing molecules, their transport, and their reactivity. Specifically, ${ }^{29} \mathrm{Si}$, ${ }^{129} \mathrm{Xe}$, and ${ }^{15} \mathrm{~N} N M R$ will be employed in conjunction with high resolution adsorption, HRADS, with DTA-TGA, and with FTIR for the initial studies of the adsorption of C6 
and $\mathrm{C7}$ cycloalkanes within ten- and twelve-member ring zeolites. In addition, the rate of adsorption/diffusion will be quantified by solids-gas chromatography (SGC). The cracking and isomerization reaction of these cycloalkanes will be studied to understand the symbiotic relationship between dynamic pore/adsorbate interactions and the resultant reactions of these cycloalkanes.

\section{University of Memphis Memphis, TN 38152}

\section{Department of Chemistry}

\section{Towards Computer Aided Catalyst Design: Three Effective Core Potential Studies of C-H Activation \\ Cundari, T.R. \\ 901-678-2629

We continue to develop and test improved approaches, in particular effective core potentials (ECPs), to computational $\mathrm{d}$ - and f-block chemistry. A joint-theory experiment study was reported for $\mathrm{XM}(\mathrm{NR})_{3}$. II-loaded complexes have been pursued in order to design more potent methane activators. The research indicates that bonding in $\mathrm{XM}(\mathrm{NR})_{3}$ is very sensitive to the nature of $\mathrm{X}$, which exerts its bonding influence through the $\sigma$ framework. Analysis suggests uses for $\mathrm{XM}(\mathrm{NR})_{3}$ as metastable precursors for $\mathrm{M}(\mathrm{NR})_{3}$ active species. Studies of tris(imido) methane activation permit an assessment of the role of $\pi$-loading, charge, geometry, and d orbital occupation. An exciting highlight concerns the role of loosely bound adducts in methane activation. Calculations suggest methane adducts which may be stable enough for experimental characterization. This study also marks introduction of a new approach in our research: molecular mechanics (MM) for high-valent d- and f-block complexes. Parameterization of MM for metals is hindered by lack of suitable force field parameters. Preliminary research indicates that ECPs can provide the metric and vibrational data for this task, thus allowing desktop modeling of metal complexes. Development of improved computational methods continues for lanthanides. Analysis of new ECPs for geometry prediction shows near quantitative agreement for all $56 \operatorname{Ln} X_{3}$ $(\mathrm{Ln}=\mathrm{Ce}-\mathrm{Lu}, \mathrm{X}=\mathrm{F}, \mathrm{Cl}, \mathrm{Br}, \mathrm{I})$. We are currently using lanthanide ECPs to probe methane activation pathways.

\section{University of Michigan Ann Arbor, MI 48109}

\section{Department of Chemistry}

\section{Hydrogen Induced $C-C, C-N$, and $C-S$ Bond Activation on Pt and Ni Surfaces Gland, J.L. 313-764-7354

Hydrogen induced reactions in absorbed organic molecules and intermediates containing $\mathrm{C}-\mathrm{S}$ and $\mathrm{C}-\mathrm{N}$ bonds on the $\mathrm{Ni}(100), \mathrm{Ni}(111)$, and $\mathrm{Pt}(111)$ single crystal surfaces are the focus of this program goal. Simple surfaces like (111) and $(100)$ have been selected so that the surface reactions kinetics for a single dominant bonding type can be studied. These studies have included both low pressure hydrogenolysis studies using TPD and vacuum spectroscopies, and in situ synchrotron based soft X-ray hydrogenolysis studies in the 0.01 torr range. In the third year of this program substantial progress has been made in the understanding of thiol and aniline hydrogenolysis on the Ni(100) and Ni(111) surfaces. Thiol hydrogenolysis is substantially more rapid on nickel than on platinum. The dominant intermediates are thiolates for methanethiol and phenylthiol on Ni. The orientation of absorbed aniline appears to be the dominant controlling factor for hydrogenolysis on both platinum and nickel. $\mathrm{Hy}-$ drogenation of the aromatic ring appears to facilitate hydrogenolysis of the $\mathrm{C}-\mathrm{N}$ bond on nickel, while on platinum direct hydrogenolysis of the $\mathrm{C}-\mathrm{N}$ bond in an aromatic intermediate appears to be dominant. The reactivity studies for metastable subsurface hydrogen with a series of simple absorbed hydrocarbons has demonstrated that hydrogenolysis is not observed for several simple hydrocarbons even with energetic hydrogen atoms coming out of the bulk; instead, selective low temperature hydrogenation reactions appear to be dominant when reaction does occur. In situ ultra soft X-ray absorption above the carbon $\mathrm{K}$ edges has been used to characterize the rates of several hydrogen addition processes primarily in amines over the past year. Temperature programmed measurements have proved particularly useful for studying the factors which control the selectivity of surface reactions over a broad range of temperature. Detailed in situ spectroscopic studies are used to characterize the surface intermediates under reaction conditions.

\section{University of Minnesota Minneapolis, MN 55455}

\section{Department of Chemical Engineering and Materials Science}

\section{Homogeneous-Heterogeneous Combustion: Chemical and Thermal Coupling Schmidt, L.D. 612-625-9391 \\ $\$ 133,000$}

The roles of homogeneous and heterogeneous reactions in combustion processes are being studied experimentally and theoretically by measuring rates and concentration and temperature profiles near reacting surfaces and by calculating these profiles for known kinetics. Laserinduced fluorescence methods are being developed to measure the concentrations of free-radical intermediates near reacting surfaces for several combustion reactions on polycrystalline platinum and rhodium as functions of surface temperatures and reactant composition, pressure, and temperature. The concentrations and internal temperatures of $\mathrm{OH}, \mathrm{NH}, \mathrm{CN}$, and other radical intermediates with and without homogeneous reaction will be measured directly. Concentration and temperature profiles are also being calculated for various reaction processes and flow conditions. Of particular interest is the occurrence of multiple steady states and oscillations for various models of homogeneous-heterogeneous processes. Reaction rate expressions for individual surface and homogeneous reactions are used to simulate the experimentally observed behavior. Particular interest centers on the selectivity of partial oxidation reaction such as production of $\mathrm{CO}$ and $\mathrm{H}_{3}$ from methane oxidation and production of formaldehyde from methanol oxidation. The objective of this 
research is to understand the contributions of each type of reaction in practical situations in catalytic reactors and combustors in order to determine their implications in reactor selectivity and pollution abatement. Rates and selectivities in reactions over $\mathrm{Pt}$ to produce $\mathrm{OH}$ and $\mathrm{HCN}$ have been compared over ceramic and metal monoliths.

\section{University of Missouri at Columbia Columbia, MO 65211}

\section{Department of Chemistry}

\section{Late Transition Metal Oxo and Imido Complexes}

Sharp, P.R.

314-882-7715

$\$ 112,687$

The synthesis and reactions of late transition metal complexes containing metal-oxygen and metal-nitrogen bonds (e.g,, oxo, imido, nitrido, and hydrazido complexes) is being studied. The goal is to develop the chemistry of these complexes as an aid to understanding the many important reactions involving late transition metal-oxygen and -nitrogen interactions and as new methods for the formation of $\mathrm{C}-\mathrm{N}$ and $\mathrm{C}-\mathrm{O}$ bonds. We have recently discovered that the gold oxo and imido complexes, $\left[(L A u)_{3}(\mu-O)\right]^{+}$ and $\left[(\mathrm{LAu})_{3}(\mu-\mathrm{NR})\right]^{+}(\mathrm{L}=\mathrm{a}$ phosphine $)$, are effective mild oxo and imido transfer reagents. The reaction of the oxo complex with [(NBD)Rh $(\mu-\mathrm{Cl}]_{2}$ yields [(NBD) $\left.\mathrm{Rh}\left(\mu-\mathrm{OAu}_{2} \mathrm{~L}_{2}\right)\right]_{2}{ }^{2+}$ where the oxo oxygen atom is in an unprecedented trigonal-pyramidal coordination environment. With (COD) $\mathrm{PtCl}_{2}$ and the gold oxo complex, $\left[(\mathrm{COD})_{4} \mathrm{Pt}_{4}(\mathrm{O})_{2}(\mathrm{OH})\right]_{3+}$ is isolated. This unusual platinum complex provides the first example of a late transition metal oxo complex capable of transferring an oxygen atom to unactivated alkenes. Major effort is being directed toward the elucidation of the requirements for transfer and the discovery of analogous imido systems and other systems capable of oxygen atom and nitrene transfer to unactivated alkenes.

\section{University of North Carolina at Chapel Hill Chapel Hill, NC 27599}

\section{Department of Chemistry}

\section{Mechanistic Studies of Transition Metal-Catalyzed Alternating Copolymerizations of Carbon Monoxide with Olefins \\ Brookhart, $M$. \\ 919-962-0362

The basic objective of this program is to elucidate the fundamental mechanisms of $\mathrm{Pd}(\mathrm{II})-$ and Ni(II)-catalyzed alternating copolymerizations of carbon monoxide with olefins. Specifically, well-defined organometallic precursors that yield active catalyst systems are being investigated to determine the catalyst resting state, details of the carbon-carbon coupling processes, the kinetics of the chain growth, and the modes of chain termination and chain transfer. Transient intermediates in the catalytic cycle are being prepared by low-temperature studies and through independent low temperature synthesis. Extensive results have been obtained using the well-defined initiator (phen) $\mathrm{Pd}\left(\mathrm{CH}_{3}\right)\left(\mathrm{OEt}_{2}\right)^{+} \mathrm{BAr}_{4}^{\prime}{ }^{-}$(phen=phenathroline, $\left.\mathrm{Ar}^{\prime}=3,5-\left(\mathrm{CF}_{3}\right)_{2} \mathrm{C}_{6} \mathrm{H}_{3}{ }^{-}\right)$. This is an active catalyst for the copolymerization of ethylene and carbon monoxide. Key intermediates in the catalytic cycle have been generated at low temperatures, and kinetics of their migratory insertion behavior has been examined. These intermediates include (phen) $\mathrm{Pd}\left(\mathrm{CH}_{3}\right)(\mathrm{CO})^{+}$, (phen) $\mathrm{Pd}\left(\mathrm{CH}_{3}\right)\left(\mathrm{C}_{2} \mathrm{H}_{4}\right)^{+}$and (phen)Pd( $\left.\mathrm{COCH}_{3}\right)\left(\mathrm{C}_{2} \mathrm{H}_{4}\right)^{+}$. Relative binding affinities of $\mathrm{CO}$ and $\mathrm{C}_{2} \mathrm{H}_{4}$ have been determined and a complete catalytic cycle has been constructed including kinetics for each step and relative populations of key species. Significant progress is also being made regarding detailed mechanistic studies of ethylene and $\alpha$-olefin polymerizations employing bidentate phosphorous ligands $\mathrm{R}_{2} \mathrm{P}\left(\mathrm{CH}_{2}\right)_{\mathrm{n}} \mathrm{PR}_{2}$. These catalytic systems have been chosen for study since they provide an opportunity to study the most fundamental details of carbon-carbon bond formation via migratory insertion reactions and because insights gained should guide the design of new catalysts for this potentially important class of polymers. A future objective of this program is to devise new catalysts for the synthesis of new copolymers based on mechanistic information acquired.

\section{Reductive Coupling of Carbon Monoxide to $C_{2}$ Products Templeton, J.L. 919-966-4575

Nitrene transfer from cationic tungsten nitrene monomers to trimethylphosphine has been accomplished. In a successful effort to increase the electrophilic character of the metal nitrene unit in $\mathrm{M}=\mathrm{N}-\mathrm{R}$ complexes a tosyl substituent was introduced. Reaction of $\mathrm{Tp}^{\prime} \mathrm{W}(\mathrm{CO})_{3} \mathrm{H}$ with tosyl azide ( $\mathrm{TsN}_{3}$ ) yields $\mathrm{Tp}^{\prime}(\mathrm{CO})_{2} \mathrm{WNHTs}$, which can be oxidized with silver salts or iodine to form the cationic nitrene complex, $\left[\mathrm{Tp}^{\prime}(\mathrm{CO})_{2} \mathrm{WNTs}^{+}\right.$. Addition of hydride from $\mathrm{BH}_{4}{ }^{-}$reforms Tp ${ }^{\prime}(\mathrm{CO})_{2}$ WNHTs while addition of $\mathrm{PMe}_{3}$ to the cation yields $\mathrm{Me}_{3}$ PNTs. These results are encouraging for developing systems that will transfer the neutral nitrene NR fragment to electron-rich olefins to form aziridines. Another significant result in $\mathrm{M}-\mathrm{N}-\mathrm{C}$ reaction chemistry is the selective regiochemistry that has been uncovered for electrophile addition to $\mathrm{W}=\mathrm{N}-\mathrm{CR}_{2}$ units. With an ancillary alkyne ligand in the coordination sphere, the nitrogen lone pair is one component of a 3-center-4electron bond. As such, it is available for protonation, and a coordinated imine ligand forms (M-NH $=\mathrm{CR}_{2}{ }^{+}$). In contrast, the regioselectivity of a proton addition is reversed when the nitrogen lone pair is involved in a simple 2center-2-electron bond. Protonation then occurs at the carbon site to form a nitrene ligand $\left(\mathrm{M}=\mathrm{N}-\mathrm{CHR}_{2}{ }^{+}\right)$. 


\section{Northwestern University Evanston, IL 60208}

\section{Department of Chemical Engineering}

\section{Solid-State, Surface, and Catalytic Studies of Oxides \\ Kung, H.H. 708-491-7492}

$\$ 132,000$

Multicomponent oxides are catalysts for a number of technologically important reactions, including the selective conversion of low-priced saturated hydrocarbons by oxidation (selective oxidation) to unsaturated hydrocarbons, aromatics, alcohols, aldehydes, or acids that are of much higher value, and for the removal of nitorgen oxides, which is an atmospheric pollutant from exhausts of leanburn, gasoline engines (lean $\mathrm{NO}_{\mathrm{x}}$ conversion). The emphasis of this project is to identify the properties of oxidic catalysts that determine their catalytic properties in these reactions. In selective oxidation, it was found that modification of a silica-supported vanadium oxide catalysts with phosphorus resulted in significant increases in the selectivity for the formation of maleic anhydride. Spectroscopic characterization of the samples showed the formation of a vanadium phosphorus compound (vanadyl phospate). However, a closer examination of the spectra showed that the samples of higher selectivities contained features not found in vanadyl phosphate, and the vanadium ions were in a lower average oxidation state than for the less selective samples. In lean $\mathrm{NO}_{\mathrm{x}}$ reduction, it was found that effective catalysts contained transition metal oxides highly dispersed in an inert matrix. A sample with poor dispersion functioned as a hydrocarbon combustion catalyst, but showed little $\mathrm{NO}_{\mathrm{x}}$ reduction activity. A model was proposed to explain the role of each catalyst component in the reaction mechanism.

\section{Department of Chemistry}

\section{Supported Organometallic Complexes: Surface Chemistry, Spectroscopy, and Catalysis \\ Marks, T.J. \\ 708-491-5658 \\ $\$ 106,400$ \\ (12 months)}

The project goal is to understand those chemisorptive processes responsible for dramatic enhancements in catalytic activity when actinide, lanthanide, and early transition element complexes are adsorbed on certain inorganic surfaces. Surface reaction chemistry is studied by chemical and spectroscopic techniques, while catalytic transformations (e.g., olefin hydrogenation) are studied by kinetic measurements, isotopic labeling, product stereochemistry, and spectroscopy. On Lewis acid supports, $\mathrm{Cp}_{2} \mathrm{MR}_{2}$ complexes $(\mathrm{Cp}=$ cyclopentadienyl-type ligand; $\mathrm{M}=\mathrm{Th}, \mathrm{U}, \mathrm{Zr}$; $\mathbf{R}=$ alkyl group) undergo $\mathrm{R}^{-}$abstraction to yield electrophiles, highly electrophilic $\mathrm{Cp}_{2} \mathrm{MR}^{+}$species, which are shown to be active catalytic centers by CPMAS NMR spectroscopy. These species can be spectroscopically, structurally, and functionally modeled in solution by $\mathrm{Cp}_{2} \mathrm{MR}^{+} \mathrm{X}^{-}$complexes, where $\mathrm{X}^{-}$is a weakly coordinat-

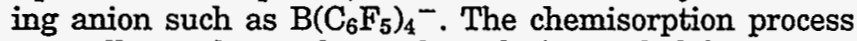
as well as the pathway by which methylalumoxane, " $\left[\mathrm{Al}\left(\mathrm{CH}_{3} \mathrm{O}\right]_{n}\right.$ " activates organo-group 4 complexes for industrial scale olefin polymerization processes can be modeled using $\mathrm{B}\left(\mathrm{C}_{6} \mathrm{~F}_{5}\right)_{3}$ and other organo-Lewis acids as abstraction reagents. Finally, these catalysts can be employed to produce completely new types of functionalized polyolefins via a ring-opening process in which exo-methylene substituted cycloalkanes open to yield exomethylene substituted polyethylenes.

\section{Chemical Interactions in} Multimetal-Zeolite Catalysts Sachtler, W.M.H.; Ipatieff, V.N. 708-491-5263

$\$ 110,321$

The problem of changing the selectivity of Rh catalysts in CO hydrogenation from hydrocarbon production to synthesis of oxygenates by "promoting" the catalyst with manganese has been addressed. By using zeolite supported samples, it was possible to synthesize samples with identical $\mathrm{Rh}$ content but with the manganese being present either as $\mathrm{Mn}^{2+}$ ions or as $\mathrm{MnO}$ particles. It was found that in the former case, the selectivity for oxygenates was $=0$; in the latter case a high yield of ethanol and ethyl acetate was obtained, suggesting that acetate ions are primary products. Subsequent catalyst characterization revealed that $\mathrm{MnO}$ and $\mathrm{Rh}$ clusters are located inside zeolite cavities and in direct physical contact with each other. As announced in our latest proposal, research towards zeolite supported acids, such as sulfated $\mathrm{ZrO}_{2}$, has been initiated.

\section{Organometallic and Surface Chemistry of Mixed-Metal Systems \\ Shriver, D.F. 708-491-5655 \\ $\$ 121,999$}

This research is focused on the reactions of ligands attached to clusters and their relation to reactions on metal surfaces. Because of their importance in surface chemistry and catalysis, most of this research concerns carbonyl, sulfur dioxide, oxo, sulfido, carbido, and hydrido ligands and their derivatives. We have demonstrated that bridging $\mathrm{CO}$ in clusters can be attached by electrophiles and subsequently converted to $\mathrm{C}, \mathrm{CCO}, \mathrm{C}_{2}$, and $\mathrm{C}_{4}$ ligands. Similarly, the attack of nucleophiles on $\mathrm{SO}_{2}$ bound to metal clusters and the conversion of coordinated $\mathrm{SO}_{2}$ to coordinated $\mathrm{S}$ or SO was demonstrated. We are currently studying the relative reactivities of bridging $\mathrm{CO}$ and $\mathrm{SO}_{2}$ ligands and extending vibrational spectroscopic characterization of the products. The latter information is relevant to the identification of ligands on metal surfaces.

\section{University of Oklahoma Norman, OK 73019}

\section{Department of Chemistry and Biochemistry}

\section{Transition Metal-Mediated Thermal and Photochemical Carbon Dioxide Activation Nicholas, K.M. $\$ 109,776$ 405-325-3696}

The overall goals of this project are to elucidate the patterns of reactivity, both thermal and photochemical, of coordinated $\mathrm{CO}_{2}$ and to develop catalytic processes based on these patterns. Following the initial discovery of the thermal and photochemical decarbonylation of $\left(\eta^{5}-\mathrm{C}_{5} \mathrm{H}_{4} \mathrm{Me}\right)_{2} \mathrm{Nb}\left(\eta^{2}-\mathrm{CO}_{2}\right) \mathrm{CH}_{2} \mathrm{SiMe}_{3}$ to 
$\left(\eta^{5}-\mathrm{C}_{5} \mathrm{H}_{4} \mathrm{Me}\right)_{2} \mathrm{Nb}(\mathrm{O}) \mathrm{CH}_{2} \mathrm{SiMe}_{3}$, investigation of the thermolyses of a series of related alkyl $\mathrm{CO}_{2}$-derivatives 1a-d ( $\mathrm{R}=\mathrm{CH}_{2} \mathrm{SiMe}_{3}, \mathrm{CH}_{2} \mathrm{CMe}_{3}, \mathrm{CH}_{2} \mathrm{Ph}, \mathrm{Me}$ ) has revealed an $\mathrm{R}-$ and concentration dependent mechanism: in dilute solution $(<0.1 \mathrm{mM}$ ) 1a-d react by a first order process to give the corresponding oxo derivatives with a modest rate dependency on the $\mathrm{R}$ group; in more concentrated solutions Ic $\left(\mathrm{R}=\mathrm{CH}_{2} \mathrm{Ph}\right)$ converts to a mixture of oxo- and carbonyl derivatives with more complex kinetics. A mechanistic rationale involves concerted $\mathrm{CO}$ extrusion for the 1st order reactions and, for 1c at higher concentrations, O-atom abstraction from $\mathrm{Cp}_{2} \mathrm{Nb}\left(\eta^{2}-\mathrm{CO}_{2}\right) \mathrm{R}$ by 16 electron $\mathrm{Cp}_{2} \mathrm{NbR}$ (generated via $\mathrm{CO}_{2}$ dissociation) via a binuclear $\mu-\mathrm{CO}_{2}$ intermediate. Other group 5 analogues (V,Ta) of $\mathrm{Cp}_{2} \mathrm{Nb}\left(\eta^{2}-\mathrm{CO}_{2}\right) \mathrm{R}$ (1) have been prepared, providing the first complete set of $\mathrm{CO}_{2}$ complexes from the same triad. During the mechanistic studies of the $\mathrm{Cp}_{2}^{\prime}{ }_{2} \mathrm{MBA}\left(\mathrm{CO}_{2}\right) \mathrm{R}$ thermolysis the remarkable aerobic oxygenation of $\mathrm{Cp}_{2}^{\prime} \mathrm{Nb}(\mathrm{CO}) \mathrm{R}(2)$ to $\mathrm{Cp}_{2}^{\prime}{ }_{2} \mathrm{Nb}\left(\eta^{2}-\mathrm{CO}_{2}\right) \mathrm{R}$ and $\mathrm{Cp}_{2} \mathrm{Mo}(\mathrm{CO})$ to $\mathrm{CpMo}\left(\eta^{2}-\mathrm{CO}_{2}\right)$ was discovered, the first arrested cases of $\mathrm{CO}$ oxidation on a metal. Extension of the aerobic oxygenation to metal carbonyl hydrides, $\mathrm{Cp}^{\prime}{ }_{2} \mathrm{Nb}(\mathrm{CO}) \mathrm{H}$ (3) and $\mathrm{Cp}_{2}^{\prime} \mathrm{Ta}(\mathrm{CO}) \mathrm{H}(4)$, has led to the generation of novel, unstable metal formato derivatives, $\mathrm{Cp}_{2} \mathrm{M}(\mathrm{O})\left(\mathrm{O}_{2} \mathrm{CH}\right)(5,6)$, which have been spectroscopically characterized. Lowtemperature NMR monitoring of the oxygenation of the $\mathrm{Ta}$ complex indicates the intermediacy of the unique $\mathrm{Cp}_{2}^{\prime} \mathrm{Ta}\left(\eta^{2}-\mathrm{CO}_{2}\right) \mathrm{H}$ species, the first hydrido-metal- $\mathrm{CO}_{2}$ complex. The analogous reaction of 2 with $S_{8}$ was found to produce the first crystallographically characterized COS complex, $\mathrm{Cp}_{2}^{\prime} \mathrm{Nb}\left(\eta^{2}(\mathrm{C}, \mathrm{S})-\mathrm{COS}\right) \mathrm{CH}_{2} \mathrm{SiMe}_{3}(8)$. This transformation of coordinated $\mathrm{CO}$ to $\mathrm{COS}$ is the first homogeneous example of this reaction and provides a model for the key step in the heterogeneously-catalyzed manufacture of COS.

\section{Pennsylvania State University, University Park University Park, PA 16802}

\section{Department of Chemical Engineering}

\section{Enhancement of Activity and Selectivity by Metal-Support Interactions \\ Vannice, $M . A$. \\ 814-863-4803

The project objectives have been (1) to study the reaction of metal-support interactions that have a pronounced influence on adsorption and catalytic behavior, and (2) to use these effects to alter hydrogenation reactions such as those involved in fine chemicals production and to enhance low-temperature CO oxidation. Focus is on characterizing adsorbed molecules as well as the chemical and physical state of the metal and support. Acetophenone hydrogenation over $\mathrm{Pt}$ showed enhanced selectivity to phenylethanol from $70 \%$ with $\mathrm{Pt} / \mathrm{SiO}_{2}$ to over $95 \%$ with $\mathrm{Pt} / \mathrm{TiO}_{2}$. Benzaldehyde hydrogenation on $\mathrm{Pt} / \mathrm{TiO}_{2}$ has turnover frequencies 10-100 times higher than those on $\mathrm{Pt} / \mathrm{SiO}_{2}$ and $\mathrm{Pt} / \mathrm{Al}_{2} \mathrm{O}_{3}$ whereas hydrogenation of benzyl alcohol is suppressed. This results in $100 \%$ selectivity to benzyl alcohol up to conversions of $80 \%$ or more. Special active sites at the metal-support interface are proposed to explain this behavior. $\mathrm{Au} / \mathrm{TiO}_{2}$ catalysts have been found to be excellent $\mathrm{CO}$ oxidation catalysts at $300 \mathrm{~K}$ and lower.
The elimination of $\mathrm{Cl}$ does not increase activity, $\mathrm{H}_{2} \mathrm{O}$ vapor reversibly decreases activity, and UHP Au powder has no detectable activity at these conditions. In situ DRIFTS measurements have identified reversibly adsorbed $\mathrm{CO}$ on the $\mathrm{Au}$, but none on the $\mathrm{TiO}_{2}$ surface, while spectator carbonate species develop on the $\mathrm{TiO}_{2}$. A kinetic model invoking noncompetitive adsorption on two types of sites located at the metal-titania interface explains the data well. An ultrahigh vacuum system with high-resolution electron energy loss spectroscopy, X-ray photoelectron spectroscopy, Auger-electron spectroscopy and a highpressure reaction chamber have been used to investigate $\mathrm{CO}$ and acetone adsorbed on a UHP Pt foil. Both $\eta^{1}$ (endon bonding) and $\eta^{2}$ (side-on bonding) species are present on clean $\mathrm{Pt}$, but neither was detected on a $\mathrm{Pt}$ surface partially covered with $\mathrm{TiO}_{\mathrm{x}}$ although decomposition products were observed. The hydrogenation of mixtures of polyaromatic hydrocarbons is being investigated to determine if the presence of acid sites on the support, which are supplied by spilled-over hydrogen, can alter selectivity during the hydrogenation of benzene/toluene feed mixtures.

\section{Department of Chemistry}

\section{Transition-Metal-Mediated Transformations of Small Molecules Sen, $A$. 814-863-2460 \\ $\$ 106,000$}

Catalytic transformations by transition metals and their compounds is of fundamental scientific, as well as practical, importance because of the high efficiency, high specificity, and low energy demands usually associated with such systems. The current research is focused on transition metal catalyzed polymerizations. The study specifically encompasses homogeneous metal catalyzed systems for the synthesis of several different kinds of copolymers incorporating the carbonyl functionality in the backbone. The carbonyl functionality is derived form carbon monoxide, an inexpensive monomer. Such polymers are of great current interest because of their photodegradability and because they are precursors to a wide range of functionalized polymers. The principal research goals are: (a) the design of living copolymerization systems that would allow the directed synthesis of block terpolymers involving the copolymerization of two different olefins with carbon monoxide, as well as block polymers incorporating polyolefin and olefin-carbon monoxide blocks, (b) the synthesis of regiospecific, stereospecific and, ultimately, chiral alternating olefin-carbon monoxide copolymers using appropriate catalysts, (c) the synthesis of star, comb, and graft polymers with alternating olefin-carbon monoxide segments, (d) the synthesis of alternating copolymers of functionalized olefins with carbon monoxide, and finally (e) the direct synthesis of polycarbonates and polyoxalates from carbon monoxide and diols.

\section{Intercollege Materials Research Laboratory}

\section{Carbon Deposition and Deactivation of Metallic Catalysts \\ Baker, T.K.

814-863-1439

The aim of this project is to develop a fundamental understanding of the manner by which certain additives modify the catalytic behavior of selected metals. The growth of 
carbon nanofibers from the metal catalyzed decomposition of ethylene in conjunction with the gas phase product distribution provides an ideal reaction probe of the chemical composition of the active surfaces and morphological characteristics of the catalyst particles. Studies of the influence of copper additions to cobalt and iron have shown that although these respective components exhibit only limited miscibility in each other, the reaction of the bimetallics with ethylene resulted in higher carbon yields and altered selectivities compared to that observed with the single metals. This behavior is rationalized according to the notion that the presence of a small amount of copper is capable of inducing electronic perturbations in the host metal atoms, which results in a modification in the chemisorption characteristics of ethylene on such a surface. During interaction with hydrocarbons, metal particles adopt a morphology where there are at least two distinct faces: a set that is only capable of adsorbing and decomposing hydrocarbons and others that will precipitate dissolved carbon in the form of graphite platelets. Under such circumstances there are a set of faces that are completely free of deposited carbon and available for continued catalytic action.

\section{Department of Materials Science and Engineering}

\section{Determination of the Distribution of Hydrogen in Coal by Fourier Transform Infrared (FTIR) Spectroscopy Painter, P.C. 814-865-5972 \\ $\$ 105,229$}

The purpose of this research is to determine the role of hydrogen-containing functional groups in coal. The work consists of two interrelated parts: (1) the quantitative determination of the aliphatic and aromatic $\mathrm{CH}$ content by Fourier transform infrared (FTIR) spectroscopic measurements and (2) the application of a newly developed thermodynamic model that describes the role of hydrogenbonding interactions. This research is aimed at providing a knowledge of coal structure both at the level of local composition and at the larger scale of the effect of the balance of intermolecular forces on solution and swelling behavior. In recent work a new model for swelling has been developed that discards the affine deformation assumption of the old Flory-Rehner approach and uses the blob model of DeGennes to obtain an expression for the free energy. It has been determined that energetic as well as entropic factors are important in terms of coal swelling in various solvents. Furthermore, some very unusual results have been obtained using mixed solvents, so that the model is now being extended in order to describe ternary systems. A new FTIR methodology has been developed to determine interaction parameters used in this model.

\section{University of Pennsylvania Philadelphia, PA 19104}

\section{Department of Chemical Engineering}

\section{Support Effects Studied on Model Supported Catalysts \\ Gorte, R.J. 215-898-4439}

The research is aimed at understanding metal/oxide and oxide/oxide interfaces for purposes of understanding catalytic support effects. The systems under investigation are noble-metal films on ceria and lanthana and ceria films on zirconia; these systems find application in automotive, emissions-control catalysis and solid-oxide fuel cells. A variety of techniques are being applied to understanding how the oxide support affects the catalytic properties. First, surface investigations are being used to characterize the structure and adsorption properties of model catalysts. This involves the use of temperature programmed desorption, transmission electron microscopy, and ion scattering on flat oxides onto which catalytic species have been deposited by vapor deposition. Oxide crystals of various orientation and polycrystalline films are used to determine how the oxide structure affects the properties. Second, catalytic investigations, including mechanistic studies of $\mathrm{CO}$ oxidation at near-atmospheric pressures and studies of model solid-oxide fuel cells, are being used to compare the catalytic properties to the adsorption properties.

\section{Department of Chemistry}

\section{Catalytic Synthesis of Silicon Carbide Preceramic Polymers: Polycarbosilanes Berry, D.H. $\$ 94,000$ 215-898-2705}

New systems have been developed for the catalytic dehydrogenative synthesis of $\mathrm{Si}-\mathrm{C}$ and $\mathrm{Si}-\mathrm{Si}$ bonds and applied to organosilicon products such as polycarbosilanes, polysilanes, and aryl silanes. Ruthenium complexes $\left(\mathrm{Me}_{3} \mathrm{P}\right)_{3} \mathrm{Ru}(\mathrm{H})_{3}\left(\mathrm{SiR}_{3}^{\prime}\right)$ catalyze the dehydrogenation of tertiary silanes to produce a distribution of linear and branched carbosilanes. The key $\mathrm{C}-\mathrm{H}$ functionalization step is achieved by $\beta$-hydrogen elimination from silyl ligands to generate transient metal silaolefin complexes of the type of $L_{\mathrm{n}} \mathrm{Ru}\left(\beta^{2}-\mathrm{R}_{2} \mathrm{Si}=\mathrm{CR}_{2}^{\prime}\right)$. A stable silene complex, $\left(\mathrm{Me}_{3} \mathrm{P}\right)_{3}\left(\mathrm{H}_{2}\right) \mathrm{Ru}\left(\beta^{2}-\mathrm{Me}_{2} \mathrm{Si}=\mathrm{CH}_{2}\right)$, has been prepared and characterized in solution and found to react rapidly with $\mathrm{HSiR}_{3}$ to stoichiometrically yield carbosilanes. In the presence of hydrogen acceptors such as $t$-butyl ethylene catalytic dehydrocoupling is rapid at $80-100^{\circ} \mathrm{C}$. Secondary silanes such as $\mathrm{Me}_{2} \mathrm{SiH}_{2}$ undergo concurrent 1,1dehydrogenative coupling and isomerization to yield branched polysilanes $\left[\mathrm{SiMe}_{2}\right]_{x}\left[\mathrm{Si}(\mathrm{Me})\left(\mathrm{SiMe}_{3}\right]_{y}\right.$. A new class of catalysts, $\left(\eta^{6}\right.$-arene $) \mathrm{Ru}\left(\mathrm{SiR}_{3}\right)_{2}(\mathrm{H})_{2}$, has also been synthesized and characterized and shown to be active for the catalytic dehydrogenative cross-coupling of $\mathrm{Si}-\mathrm{H}$ and Ar- $\mathrm{H}$ bonds to yield aryl silanes. Current studies are aimed at increasing the selectivity of $\mathrm{Si}-\mathrm{C}$ and $\mathrm{Si}-\mathrm{Si}$ bond forming processes, understanding reactions that lead to catalyst degradation, and extending the chemistry to other organosilicon products and other main group element polymers of interest. 


\section{Inorganic Polymers and Materials} Sneddon, L.G. 215-898-8632

This project is focused on the development of new polymeric-precursor synthetic routes that enable the formation of a variety of nonoxide ceramics in processed forms. Current studies are conducted on the syntheses, properties, ceramic-conversion reactions, and applications of boron-based polymers, including polyvinylborazine, polyborazylene, borazine-modified polysilazanes, and decaborane-based polymers. In addition, investigations are conducted on the use of these polymers as reagents for the synthesis of a wide range of metal boride, metal nitride, and metal silicide ceramics. A major achievement of the last year has been the development of the first meltspinnable polymeric precursors to SiNCB ceramic composite fibers. The continued development of the fundamental synthetic methodology needed to produce new inorganic monomers and polymers is also a key component of this project.

\section{Catalytic Hydrogenation of Carbon Monoxide \\ Wayland, B.B. \\ 215-898-8633}

$\$ 127,885$

A central objective of this program is to explore and develop new strategies for reduction and hydrogenation of carbon monoxide at mild conditions of pressure and temperature. One prominent pathway for hydrogenation of $\mathrm{CO}$ to form organic oxygenates passes through a metalloformyl intermediate that is a participant in the subsequent rate limiting process. Metalloformyl species (M-CHO) are generally thermodynamically unfavorable relative to the metal hydride (M-H) and CO precursors and thus, very high pressures of $\mathrm{CO}$ are required to form finite quantities of this central intermediate. The seminal observation that rhodium porphyrin complexes have the thermodynamic capability to produce formyl species at low pressures of $\mathrm{H}_{2}$ and $\mathrm{CO}$ is utilized in guiding the design of metal complexes that have both the thermodynamic and kinetic properties necessary for catalytic CO hydrogenation. Production of formyl species from $\mathrm{H}_{2}$ and CO has now been shown to be a general property for rhodium complexes of nitrogen donor macrocycles and most recently also was observed for nonmacrocyclic tetradentate ligands with both nitrogen and oxygen donors. Structurally flexible nonmacrocyclic ligand complexes manifest reaction pathways excluded to macrocyclic ligand complexes, and have the capability of achieving oxidative addition, reductive elimination, and migratory insertion steps integral to selective $\mathrm{CO}$ hydrogenation to alcohols. An alternate reaction pathway to form organic oxygenates that occurs through initial $\mathrm{CO}$ reductive coupling has also been observed for rhodium porphyrins and the scope of rhodium complexes that can reduce and couple $\mathrm{CO}$ is currently being evaluated.

\section{University of Pittsburgh Pittsburgh, PA 15261}

\section{Department of Chemical and Petroleum Engineering}

\section{Fundemental Aspects of Selective Reduction of $N \mathrm{O}_{x}$ and Low Temperature Methane Activation Catalyzed by Zeolites d'Itri, J.L.; Hall, K.W. 412-624-9634 \\ $\$ 142,000$}

Medium pore-sized zeolites containing a wide variety of charge-compensating cations are active catalysts for selective reduction of NO by hydrocarbons and for lowtemperature acidification of $\mathrm{CH}_{4}$ by NO. The objective of the proposed research is to develop a fundamental understanding of the sites responsible for both of these chemical processes and the reaction mechanism. Metal-loaded zeolites will be prepared by ion exchange under controlled conditions that will be systematically changed in order to vary the type and concentration of metal species. The catalysts are to be characterized by a variety of techniques including FTIR, MAS-NMR, temperature-programmed reactions with probe molecules, and isotopic exchange reactions. Fundamental information regarding the individual steps involved in the reaction mechanism and which of these steps are rare limiting will be developed by isotopic transient investigations. The role of parameters such as zeolite acidity, structure, and the reducibility of the charge compensating will be probed through reaction studies and catalyst characterization, and relationships will be developed between these catalyst properties and key reaction steps such as the activation of methane. Moreover, the importance of components such as HCN and $\mathrm{NO}_{2}$ in forming a $\mathrm{N}-\mathrm{N}$ bond in these systems will be investigated.

\section{Department of Chemistry}

\section{Vibrational Spectroscopic Studies of Surface Chemical Interactions in Chemisorption and Catalysis Yates, J.T., Jr.} 412-624-8320

Catalytic reactions are being studied using vibrational spectroscopy methods coupled with electron and photon stimulation of the reactions. The vibrational spectroscopic methods include Fourier transform infrared reflection absorption spectroscopy (IRAS) on model single-crystal catalysts and transmission infrared spectroscopy (TIR) on high area powdered catalysts. Current problems include: (1) study of the role of defect sites on catalytic reactions; (2) study of the activation of chemical bonds by coordinatively unsaturated surface sites on supported metal catalysts; and (3) study of the dynamical motion of adsorbates on single-crystal surfaces and the anisotropic nature of these motions. A particularly important objective is the use of photodesorption methods to produce vacant binding sites on anchored metal carbonyls, which then have the property of activating C-H bonds. Both methane and cyclohexane have been activated using these sites to break C-H bonds at low temperatures. This work will be extended to the thermal activation regime to determine if a practical 
route for $\mathrm{C}-\mathrm{H}$ bond activation may be developed using the principles discovered by photochemical methods.

\section{Purdue University \\ West Lafayette, IN 47907}

\section{Department of Chemistry}

\section{Novel Intrazeolite Metal-Oxo Catalysts and Alloy Clusters \\ Bein, $T$. 317-494-5495

The reactivity of metal-organic compounds and organometallics in zeolite cages is studied, targeting, the preparation of novel hybrid catalysts with unusual shape selectivity. The intrazeolite chemistry of the precursor compounds, their thermal chemistry, reactivity, and catalytic activity and selectivity are examined using techniques such as EXAFS, in situ FTIR coupled to thermodesorption, Raman, UV-NIR, and ESR. In current work, methyltrioxorhenium (MTO) is being encapsulated in different forms of zeolite $Y$ having a range of acidity levels. EXAFS and vibrational studies show that at room temperature, the complex maintains its integrity in the hosts, but associates with acidic groups. The MTO-zeolite adducts are active in the metathesis of 1-hexene at room temperature, in competition with double bond isomerization and secondary metathesis products. The MTO molecule is activated by the intrazeolite protons, and simultaneously blocks their isomerization activity. In another recent study, vanadium oxo species are introduced into the channels of zeolites and the mesoporous MCM-4I host via adsorption and decomposition of different molecular precursors. EXAFS data of $\mathrm{VOCl}_{3}$ in acidic $\mathrm{Y}$ zeolite show that the chloride ligands are removed on adsorption at room temperature. Oxovanadium species attach into the framework via oxo-bridges on calcination. The Vzeolites effectively catalyze the partial oxidation of alkyl-alcohols with hydrogen peroxide at moderate temperatures.

\section{Catalytic Arene Hydrogenation Using Early Transition Metal Hydride Compounds \\ Rothwell, I.P. 317-494-7012}

$\$ 115,000$

During the last year a concerted effort has been focused on gaining a better understanding of the mechanism of arene hydrogenation catalyzed by Group 5 metal hydride compounds. The kinetics of the hydrogenation of arylphosphines (e.g., $\mathrm{PPh}_{3}, \mathrm{RPPh}_{2}$ ), etc. by niobium aryloxide systems has been studied and successfully modeled. The stereochemistry of the reactions have been explored by a variety of methods and shown to occur in a predominantly all-cis fashion. The stoichiometric reaction of tantalum polyhydrides, $\left[\mathrm{Ta}(\mathrm{OAr})_{2}(\mathrm{Cl})(\mathrm{H})_{2}(\mathrm{~L})_{\mathrm{x}}\right] \quad(\mathrm{x}=1,2)$, and $\left[\mathrm{Ta}(\mathrm{OAr})_{2}(\mathrm{H})_{3}(\mathrm{~L})_{2}\right]$ with cyclohexene, cyclohexadiene and related olefinic substrates has been surveyed. The organometallic products obtained are found to be highly dependent on the nature of the aryloxide ancillary ligation. The mechanism of styrene hydrogenation by $\left[\mathrm{Ta}(\mathrm{OAr})_{2}(\mathrm{Cl})(\mathrm{H})_{2}\left(\mathrm{PMe}_{2} \mathrm{Ph}\right)_{2}\right](\mathrm{OAr}=2,6-$ diphenylphenoxide) is being studied. The regio and stereoselectivities of various surface supported Group 5 metal species are also being evaluated. In particular, the nature of the oxide support on the hydrogenation of arene substrates is being studied.

\section{Fundamental Studies of Reactive Intermediates in Homogeneous Catalysis Squires, R.R: 317-494-7322 \\ $\$ 77,000$}

The thermodynamic properties of organic and organometallic species involved in combustion and homogeneous catalysis are being investigated with a selected ion flow tube-triple quadrupole apparatus. Accurate values for the sequential M-CO bond strengths in an extensive series of transition metal carbonyl ions, $\mathrm{M}(\mathrm{CO})_{\mathrm{n}}{ }^{-}$, and their cyclopentadienyl derivatives, $\mathrm{CpM}(\mathrm{CO})_{n}{ }^{+/-}\left(\mathrm{Cp}=\mathrm{c}-\mathrm{C}_{5} \mathrm{H}_{5}\right)$ through energy-resolved tandem mass spectrometry has been determined. A clear picture of the periodic, homologous, and isoelectronic trends in the magnitudes of $\mathrm{M}-\mathrm{CO}$ bond strengths is emerging from these measurements. Absolute heats of formation for a variety of radicals, carbenes, and biradicals has been measured. These data are essential for understanding $\mathrm{C}-\mathrm{H}$ bond energies in hydrocarbons. A new method for preparing intense. beams of structurallydefined radical ions has been developed, which is suitable for use in photoelectron studies of carbenes and biradicals. The utility of electrospray ionization for investigating catalytically-active homogeneous. solutions is currently being evaluated. The construction of a new flowing afterglow-guided ion beam instrument employing a tandem quadrupole-octopole-quadrupole analyzer is near completion. This device will be used for refined measurements of thermochemical properties of reactive organic and organometallic intermediates.

\section{Rensselaer Polytechnic Institute Troy, NY 12180}

\section{Department of Chemistry}

\section{Selective Transformations of Carbonyl Ligands to Organic Molecules Cutler, A.R. 518-276-8447

The manganese acyl complexes $(\mathrm{L})(\mathrm{CO})_{4} \mathrm{MnC}(\mathrm{O}) \mathrm{R}(\mathrm{L}=\mathrm{CO}$, $\mathrm{PPh}_{3} ; \mathrm{R}=\mathrm{CH}_{3}, \mathrm{Ph}$ ) function as extremely active hydrosilation precatalysts toward a variety of organic carbonyl substrates. $\mathrm{Mn}\left(\mathrm{PPh}_{3}\right)(\mathrm{CO})_{4} \mathrm{C}(\mathrm{O}) \mathrm{CH}_{3}$-catalyzed $\mathrm{Me}_{2} \mathrm{PhSiH}$ hydrosilation of acetone, acetophenone, and cyclohexanone, for example, occurs under conditions $\left(1 \% \mathrm{Mn}, 22^{\circ} \mathrm{C}\right.$ in $\left.\mathrm{C}_{6} \mathrm{D}_{6} ; \mathrm{Nt}=29 \pm 3 \mathrm{~min}^{-1}\right)$ where $\mathrm{RhCl}\left(\mathrm{PPh}_{3}\right)_{3}$ is an inert. or very sluggish catalyst. The manganese carbonyl catalyzed hydrosilation-then-reduction of formate esters affords silyl acetals $\mathrm{CH}_{2}\left(\mathrm{OSiR}_{3}\right) \mathrm{OR}^{\prime}$ (several characterized) then methoxysilanes, depending on the choice of manganese precatalyst and hydrosilane. Analogous disiloxyacetals $\mathrm{CH}_{2}\left(\mathrm{OSiR}_{3}\right)_{2}$ and $\mathrm{CH}_{2}\left(\mathrm{OSiR}_{3}\right)\left(\mathrm{OSiR}_{3}^{\prime}\right)\left(\mathrm{SiR}_{3}\right.$, $\mathrm{SiR}_{3}^{\prime}=\mathrm{SiMe}_{2} \mathrm{Ph}, \mathrm{SiMe}_{2} \mathrm{Et}, \mathrm{SiHPh}_{2}$, and $\mathrm{SiH}_{2} \mathrm{Ph}$ ). are generated (and some cases characterized) from the Mncatalyzed dehydrogenative silation then hydrosilation of formic acid. These thermally sensitive disiloxyacetals undergo further Mn-catalyzed reductive hydrosilation to methoxysilanes via a pathway that does not require prion disiloxane extrusion. These studies have been carried out 
with carboxylic acids in order to characterize more stable and accessible silyl esters $\mathrm{RC}(\mathrm{O}) \mathrm{OSiR}_{3}$ and disiloxyacetals $\mathrm{RCH}\left(\mathrm{OSiR}_{3}\right)_{2}\left\{\mathrm{R}=\mathrm{Me}, \mathrm{PhCH}_{2}\right\}$. Studies on the lability of $\left(\eta^{5}\right.$-indenyl)(L)(CO)Ru-alkyl complexes have been extended to aklyl and alkene ligand isomerization reactions. Carbonylation of the alkyl complexes $[\mathrm{Ru}]-\mathrm{CH}\left(\mathrm{CH}_{3}\right) \mathrm{R}$ ([Ru] $=\mathrm{Ru}(\mathrm{CO})_{2}\left(\eta^{5}\right.$-Ind) and $\mathrm{R}=\mathrm{Me}, \mathrm{Et}, \mathrm{Pr}$ ) engender a novel carbonylation-driven iso-alkyl to $n$-acyl ligand transformation, favoring $[\mathrm{Ru}]-\mathrm{C}(\mathrm{O}) \mathrm{CH}_{2} \mathrm{CH}_{2} \mathrm{R}$. Analogous i-alkyl to n-alkyl ligand isomerization was not observed. These carbonylation-driven isomerizations apparently involve the sequential coupling of reversible $\eta^{5} / \eta^{3}$ Ind ring slippage with carbonylation and then $\eta^{3} / \eta^{1}$ Ind ring slippage with alkyl ligand isomerization steps. A series of $\eta^{1}$-alkene complexes $[\mathrm{Ru}]-\left\{\mathrm{RCH}=\mathrm{CHR}^{\prime}\right\}^{+}\left\{\mathrm{R}, \mathrm{R}^{\prime}=\mathrm{H}, \mathrm{Me}\right.$, Et, $\mathrm{Pr}, \mathrm{Bu}$ : propenes through hexenes $\}$ have been characterized. In solution, these complexes provide equilibrium mixtures of cis/trans isomers as well as shifting the double bond towards the terminal position.

\section{University of Rochester Rochester, NY 14627}

\section{Department of Chemical Engineering}

\section{Dimensional Effects in Controlled Structures Support Catalysts Derived from Layered Synthetic Microstructures (LSMs) \\ Saltsburg, $H$. \\ 716-275-4582

A new class of supported catalysts has been produced using solid-state fabrication techniques typical of the microelectronics industry. Deposition of alternating, nanometer thick layers of catalyst and support on an inert wafer, followed by etching perpendicular to the flat surfaces to reveal only the edges of the layers, provides a catalyst surface in the form of nanometer-wide, micrometer-long lines (the edge of a thin plate). These Layered Synthetic Microstructures (LSMs), with Ni and silica as catalyst and support, duplicate the size effect ("structure sensitivity") which is observed during ethane hydrogenolysis on traditional silica supported $\mathrm{Ni}$ clusters of nanometer "diameter". In principle, any catalyst/ support system can be manufactured so that the catalyst and support are uniform in size and geometry with arbitrary nanometer dimensions. Surface studies can be carried out on a totally accessible surface and one which behaves catalytically like a supported cluster. The objective of this research is to develop this new structure as a tool for understanding supported catalysts. LSMs (Ni/ $\mathrm{SIO}_{2}$ ) will be fabricated using ion milling to provide higher catalyst surface areas per unit wafer area. Other supports will be studied $\left(\mathrm{Al}_{2} \mathrm{O}_{3}\right.$ carbon, $\mathrm{MgO}$, and silicaalumina). The $\mathrm{Ni} / \mathrm{SIO}_{2}$ LSMs will be tested using other structure sensitive and structure insensitive catalytic reactions. These include the reaction of $\mathrm{CO}$ and $\mathrm{H}_{2}$ which shows a rate maximum with 4 nanometer clusters; cyclopropane hydrogenation exhibits shows a rate maximum with 2 nanometer clusters; and benzene hydrogenation which is unresolved. Characterization will be carried out concurrently. TEM can be used to examine the edge array, Auger analysis will provide a spatially averaged composition, and both STM (AFM) and TPD will be used.
Fabrication of Pt based LSMs will be carried out. Platinum catalyzed reactions which would be candidates for study include: hydrogen plus oxygen at $273 \mathrm{~K}$ since in excess hydrogen the rate is structure sensitive while in excess oxygen it is insensitive; the hydrogenation of cyclohexene which is structure insensitive; and skeletal isomerization of methylcyclopentane which exhibits selectivity changes (rather than rate changes) with cluster size greater than 2 nanometers.

\section{Department of Chemistry}

\section{Transition Metal Activation and Functionalization of Carbon-Hydrogen Bonds}

Jones, W.D. 716-275-5493

The investigation of homogeneous $\mathrm{C}-\mathrm{H}$ bond activation has been continued with a variety of metal complexes. The reactive fragment $\left[\mathrm{Cp} * \mathrm{Rh}\left(\mathrm{PMe}_{3}\right)\right]$ has been found to react with a variety of alkanes and arenes to give $\mathrm{C}-\mathrm{H}$ oxidative addition products, and with fused polycyclic hydrocarbons to give eta-2 complexes and/or C-H bond activation products. The project has now been expanded to include C-C bond activation. Reaction of this same fragment with biphenylene results in aryl-aryl bond cleavage and the formation of rhodium biaryl complex. Mechanistic studies indicate that this product is a rhodium biaryl complex formed via initial aromatic $\mathrm{C}-\mathrm{H}$ bond oxidative addition followed by intramolecular rearrangement to the C-C inserted product. A homogeneous catalyst has also been found for the hydrogenolysis of the aryl-aryl bond of biphenylene, giving biphenyl. C-C cleavage reactions of biphenylene have also been observed with other rhodium and cobalt complexes. We have also examined reactions of a series of trispyrazolylborate complexes of rhodium. The alkane and arene activation products [HB(3,5dimethylpyrazolyl $\left.)_{3}\right] \mathrm{Rh}(\mathrm{CNR})\left(\mathrm{R}^{\prime}\right) \mathrm{H}_{2}$ where $\mathrm{R}=$ neopentyl have been studied. Competitive activation of alkanes has been examined, showing that this fragment is more selective than either $\mathrm{Cp}^{*} \mathrm{Rh}\left(\mathrm{PMe}_{3}\right)$ or $\mathrm{Cp}{ }^{*} \operatorname{Ir}\left(\mathrm{PMe}_{3}\right)$. Methane has been activated thermally at 2000 psi. Reaction with cyclopropane initially gives a $\mathbf{C}-\mathrm{H}$ activation product, which then converts intramolecularly to a four-membered metallacycle product. Ethylene initially reacts by way of vinylic $\mathrm{C}-\mathrm{H}$ activation, but then converts intramolecularly to an eta-2 ethylene complex.

\section{Rutgers, The State University of New Jersey Piscataway, NJ 08855}

\section{Department of Chemistry}

\section{Carbon-Hydrogen Bond Functionalization Catalyzed by Transition-Metal Systems Goldman, A.S. 908-932-5232 \\ $\$ 90,000$}

Significant progress has been made toward the goal of effcient transition-metal catalyzed alkane functionalization, particularly dehydrogenation. Based on the results of mechanistic studies of alkane photodehydrogenation catalyzed by $\mathrm{Rh}\left(\mathrm{PMe}_{3}\right)_{2}(\mathrm{CO}) \mathrm{Cl}$, complexes of the 
form $\mathrm{Rh}\left(\mathrm{PMe}_{3}\right)_{2} \mathrm{ClL}$ have been discovered to catalyze efficient thermal (non-photochemical) alkane transferdehydrogenation. Apparently, the role of hydrogen is to add to the complexes which then dissociate $L$ to afford $\mathrm{H}_{2} \mathrm{Rh}\left(\mathrm{PMe}_{3}\right)_{2} \mathrm{Cl}$ which then reacts with hydrogenacceptors to give the fragment $\mathrm{Rh}\left(\mathrm{PMe}_{3}\right)_{2} \mathrm{Cl}$; the latter then reacts with alkanes. The development of hydrogenfree systems has also been explored. $\mathrm{Rh}\left(\mathrm{PiPr}_{3}\right)_{2} \mathrm{Cl}$ was found to react in cyclooctane solution to give $\mathrm{H}_{2} \mathrm{Rh}\left(\mathrm{Pi}^{\mathrm{i}} \mathrm{Pr}_{3}\right)_{2} \mathrm{Cl}$ (ca. $60 \%$ yield) and cyclooctane. In the presence of hydrogen acceptors, catalytic transferdehydrogenation is observed. The efficiency of both the stoichiometric and catalytic reactions of $\mathrm{Rh}\left(\mathrm{P}^{\mathrm{i}} \mathrm{Pr}_{3}\right)_{2} \mathrm{Cl}$ is limited by ligand dehydrogenation reactions. Future work in this area will focus in part on the development of complexes $\mathrm{RhL}_{2} \mathrm{C} 1$ where $\mathrm{L}^{\prime}$ has properties similar to that of ${ }^{\mathrm{P}} \mathrm{Pr}_{3}$ but is more resistant to dehydrogenation. Attempt to remove $\mathrm{L}$ from $\mathrm{Rh}\left(\mathrm{PMe}_{3}\right)_{2} \mathrm{ClL}$, by using oxidants instead of light or $\mathrm{H}_{2}$, have led to the oxidation of ligand $\mathrm{C}-\mathrm{H}$ bonds; this may be a starting point for hydrocarbon oxidation catalysis. High-level ab initio MO calculations have been successfully used to model the effect of varying ligands on the thermodynamics and kinetics of $\mathrm{H}_{2}$ and $\mathrm{C}$ $\mathrm{H}$ bonds in addition to late transition metal complexes; the results should be applicable to a wide range of catalytic reactions including, but much broader than, $\mathrm{RhL}_{2} \mathrm{C1}$-catalyzed alkane dehydrogenation.

\section{Department of Physics and Astronomy}

\section{Morphological Instability in Model Thin Film Catalysts: Structure and Reactivity Madey, T.E. 908-445-5185

The goal of this work is to probe the relationship between microscopic surface structure and catalytic activity of model bimetallic catalysts, i.e., ultrathin films of metals on metal substrates. Recent studies have focused on the structure, reactivity, and electronic properties of atomically-rough W(111) and $\mathrm{Mo}(111)$ surfaces covered by ultrathin films of various transition metals. LEED has been used in an extensive survey to identify which metals induce faceting and which do not. A striking materials specificity is found: (112) oriented facets are formed on W(111) and $\mathrm{Mo}(111)$ by ultrathin films of $\mathrm{Rh}, \mathrm{Pd}, \mathrm{Ir}, \mathrm{Pt}$, and $\mathrm{Au}$, upon annealing in the range $700 \mathrm{~K}$ to ca. $1100 \mathrm{~K}$. In contrast, $\mathrm{Ti}, \mathrm{Mn}, \mathrm{Fe}, \mathrm{Co}, \mathrm{Ni}, \mathrm{Cu}, \mathrm{Ag}$, and $\mathrm{Gd}$ do not induce facets. These observations lead to a correlation between faceting and electronic properties: only elements with Pauling electronegativity $>2.0$ cause faceting. To probe electronic effects in interface formation, high resolution soft x-ray photoemission spectroscopy (SXPS) of the bimetallic systems is being carried out using synchrotron radiation at the NSLS; surface core-level shifts are measured and associated with the planar-to-facet transition. Microscopic details of metal-induced faceting are verified using an ultrahigh vacuum scanning tunneling microscope, where the dependences of facet formation on metal coverage and temperature are observed. Finally, the catalytic activity of planar and faceted surfaces is studied using a structure-sensitive reaction, $\mathrm{n}$-butane hydrogenolysis. Reaction rates and product distributions depend strongly on the presence or absence of (112) facets, and on the facet sizes.

\section{University of South Carolina Columbia, SC 29208}

\section{Department of Chemistry and Biochemistry}

\section{The Transformations of Organic Amines by Transition Metal Cluster Compounds Adams, R.D. \\ 803-777-7187 \\ $\$ 111,500$}

Recent studies have been focused on the activation of strained cyclic hydrocarbons containing sulfur by metal cluster complexes. The principal objective is to develop catalysts for the formation of polythiaether macrocycles by the ring opening cyclooligomerization of the strained ring precursors. Studies include determining the character of the activation process and the mechanisms of the cyclooligomerizations. Investigations of the ligand behavior and stability of the polythiaether macrocycles in cluster complexes are also being investigated through the preparation and crystallographic characterization of new complexes containing these ligands.

\section{University of Southern California Los Angeles, CA 90089}

\section{Department of Chemistry}

\section{Chemistry of Bimetallic and Alloy Surfaces \\ Koel, B.E. 213-740-4126}

$\$ 104,000$

The discovery of methods to prepare stable, ordered surface alloys of $\mathrm{Sn}$ and $\mathrm{Pt}, \mathrm{Pd}$, and Ni provides an enormous opportunity to make fundamental advances in understanding how chemistry is controlled on bimetallic and alloy surfaces. Angle-resolved, low-energy alkali ion scattering studies are being used to elucidate the detailed geometric structure of these surface alloys. Hydrocarbon chemisorption studies probe the reactions that occur on these surfaces. Our focus has been on the chemistry of $\mathrm{Sn} /$ Pt(111) surface alloys, where examinations are conducted on the changes that occur in dehydrogenation selectivity for the series $\mathrm{Pt}(111),(2 \times 2) \mathrm{Pt}_{3} \mathrm{Sn}(111)$ which contains three-fold Pt sites, and $(\sqrt{ } 3 \times \sqrt{ } 3) \mathrm{R}^{\circ} 0^{\circ} \mathrm{Pt} 2 \mathrm{Sn}$, which only has two-fold Pt sites. Later, the chemistry of $\mathrm{Sn} / \mathrm{Pt}(100)$ surface alloys will be explored and compared, and also the analogous $\mathrm{Sn} / \mathrm{Pd}$ and $\mathrm{Sn} / \mathrm{Ni}$ single crystal surface alloys will be studied. In parallel with chemisorption studies in UHV, hydrocarbon conversion reaction kinetics and mechanisms will be investigated over these surfaces at higher pressures, including in situ observations of catalytic reaction intermediates by Fourier transform infrared (FTIR) spectroscopy. This work will aid in developing a basic chemical foundation for the role that alloys play in $\mathrm{Pt} / \mathrm{Sn}$ reforming catalysts and may be helpful in the design of improved catalysts. 


\section{Stanford University Stanford, CA 94305}

\section{Department of Chemical Engineering}

\section{The Dynamics of Adsorption on Clean and Adsorbate-Modified Transition Metal Surfaces \\ Madix, R.J. $\$ 152,000$ 415-723-2402}

The objectives of this research are (1) to determine the probabilities of both dissociative and nondissociative adsorption of alkanes on clean and adsorbate-covered surfaces, (2) to gain an understanding of the molecular dynamics of the adsorption process, and (3) to clarify the role of precursor states in adsorption. Model metal surfaces are studied under highly controlled conditions in ultrahigh vacuum to reveal the dynamical features of the adsorption process. Molecular beams of gases are directed at these surfaces and the dependence of the adsorption probabilities for reactive and/or nonreactive adsorption are measured directly. Stochastic simulations are combined with the experiments related to nondissociative adsorption to gain insight into the energy exchange processes that lead to trapping and adsorption. Recent focus has been on the studies of the adsorption of ethane on $\mathrm{Pt}(111)$ and $\mathrm{Pt}(110)$ surfaces to determine the effects of the microroughness of the (110) surface. The molecular adsorption probabilities of ethane, propane, and xenon were determined incident along both the (100) and (110) azimuths as a function of translational energy and incident polar angle at a surface temperature of $95 \mathrm{~K}$ using molecular beams. Along the (110) direction the adsorption probability increases with increasing angle of incidence, whereas along the (100) direction it decreases with angle. To understand these observations, stochastic simulations were performed. Ethane was treated as a pseudodiatomic molecule, and the solid was represented by a three- to fivelayer slab of at least 108 platinum atoms coupled to a heat bath by friction and random forces. The methyl-methyl and the methyl-platinum forces were calculated using pairwise Morse potentials, and the platinum-platinum force constants were calculated from bulk and surface Debye frequencies. Morse parameters of $D_{e}=2.3 \mathrm{~kJ} / \mathrm{mol}, R_{0}$ $=3.1 \mathrm{~A}$, and $\mathrm{B}=1.3 \mathrm{~A}^{-1}$ gave an excellent fit. The stochastic trajectory simulations predicted the magnitude of the adsorption probability to within $20 \%$ at all incident angles and energies as well as the different dependence of the adsorption probability on polar angle at the two azimuths studied. Examination of the energy flow during the trapping process shows that excitation of rotational energy into cartwheeling motion can assist trapping, if the excitation is not excessive. The simulations suggest that ethane trapping on $\mathrm{Pt}(110)$ (2x1) is enhanced relative to $\mathrm{Pt}(111)$ because the stronger corrugation of the $\mathrm{Pt}(110)(2 \times 1)$ surface allows for more conversion of normal momentum to parallel momentum. Initial results suggest that the adsorption probabilities and the energy scaling determined experimentally for propane on $\mathrm{Pt}(111)$ are predicted by simulations using the Morse potential parameters determined from the ethane/Pt(111) system. Recently the use of these potentials in predicting the dynamics of trapping of methane and propane on Pt(111) have been explored.
Initial results suggest that the methyl-Pt two body potential indeed accounts for the measured dependence of the trapping probabilities on incident angle and energy.

\section{Department of Chemistry}

\section{Photochemistry of Intermolecular $\mathrm{C}-\mathrm{H}$ Bond Activation Reactions}

Lees, A.J.

607-777-2362

The photochemistry of the following $\mathrm{C}-\mathrm{H}$ activating organometallic systems is being investigated: $\mathrm{CpRh}(\mathrm{CO})_{2}$, $\mathrm{Cp} * \mathrm{Rh}(\mathrm{CO})_{2}, \quad \mathrm{Cp} * \operatorname{Ir}(\mathrm{CO})_{2}, \quad\left(\mathrm{HBPz} *_{3} \operatorname{Ir}(\mathrm{CO})_{2}\right.$, where $\mathrm{CP}$ $=\eta^{5}-\mathrm{C}_{5} \mathrm{H}_{5}, \quad \mathrm{Cp} *=\eta^{5}-\mathrm{C}_{5} \mathrm{Me}_{5}$, and $\mathrm{Pz} *=3,5-$ dimethylpyrazolyl. In each case the intermolecular $\mathrm{C}-\mathrm{H}$ and $\mathrm{Si}-\mathrm{H}$ bond activation reactions have been monitored in hydrocarbon and silane solutions by in situ spectroscopic measurements. Quantitative measurements (quantum efficiencies) for these photochemical processes have been obtained. The results illustrate that all the complexes are able to efficiently facilitate intermolecular $\mathrm{C}-\mathrm{H}$ bond activation of both alkanes and aromatic solvents following excitation in the near-UV region (313-366 $\mathrm{nm})$. However, for each system it has been determined that excitation within the visible region $(405-488 \mathrm{~nm})$ also leads to $\mathrm{C}-\mathrm{H}$ bond activation, albeit with considerably reduced efficiency. It appears that in each complex there are two low-lying electronically excited states that give rise to different reaction intermediates and, hence, different photochemical pathways. The photophysical processes between these two excited levels are highly influential with respect to the subsequent $\mathrm{C}-\mathrm{H}$ bond activation mechanisms. In each instance UV photolyses (313-366 nm) is understood to produce a highly reactive monocarbonyl complex that rapidly reacts with hydrocarbon solvent to efficiently form the oxidative addition complex. On the other hand, visible irradiations $(405-488 \mathrm{~nm})$ produce entirely different reaction intermediates that do not effectively undergo oxidative addition with the hydrocarbon substrate; these transient complexes are believed to involve either $\eta^{5} \rightarrow \eta^{3}(\mathrm{Cp})$ or $\eta^{3} \rightarrow \eta^{2}\left(\mathrm{HBPz} *_{3}\right)$ ligand interconversions. Currently, the photophysical and photochemical mechanisms in these key $\mathrm{C}-\mathrm{H}$ activating systems are being further investigated. 


\section{State University of New York at Buffalo Buffalo, NY 14260}

\section{Department of Chemistry}

\section{Mechanistic Examination of Organometallic Electron-Transfer Reactions \\ Atwood, J.D. 716-645-6800}

The goal of this research is to provide a mechanistic understanding of electron transfer processes between organometallic complexes to enable utilization of the reactivity of odd-electron complexes in catalytic reactions. It has been shown that metal carbonyl anions participate in single electron processes with a number of organometallic complexes and by two-electron processes with other complexes and have begun to gain an understanding of which process is expected. In the past year these studies have focused on three areas. (1) Reactions of the preformed seventeen-electron complexes $\mathrm{Cp}^{*} \mathrm{Cr}(\mathrm{CO})_{3}$. and $\operatorname{Re}(\mathrm{CO})_{3}\left(\mathrm{PCy}_{3}\right)_{2}$. with metal complexes have shown transfer of $\mathrm{H}$. and $\mathrm{Br}$. but not $\mathrm{CH}_{3}$. These transfers are not as rapid as transfer of $\mathrm{H}^{+}, \mathrm{Br}^{+}$, or $\mathrm{CH}_{3}{ }^{+}$, but are more rapid than transfers as anions $\left(\mathrm{H}^{-}, \mathrm{Br}^{-}, \mathrm{CH}_{3}^{-}\right)$. (2) Reactions of $\mathrm{Os}(\mathrm{CO})_{4}(\mathrm{H})\left(\mathrm{CH}_{3}\right)$ with a variety of metal carbonyl anions show transfer of $\mathrm{H}^{+}$forming $\mathrm{Os}(\mathrm{CO})_{4} \mathrm{CH}_{3}{ }^{-}$; no evidence is found for transfer of $\mathrm{CH}_{3}{ }^{+}$. (3) The Fischer carbene complexes, $(\mathrm{CO})_{5} \mathrm{M}=\mathrm{C}(\mathrm{OMe}) \mathrm{Ph}$, react with metal carbonyl anions in a very unusual $\mathrm{CH}_{3}{ }^{+}$transfer resulting in $\mathrm{M}(\mathrm{CO})_{5}(\mathrm{C}(\mathrm{O}) \mathrm{Ph})^{-}, \mathrm{M}=\mathrm{Cr}, \mathrm{W}$. This reaction is the reverse of the carbene formation reaction. The acyl anion has a nucleophilicity only slightly more than $\mathrm{Co}(\mathrm{CO})_{4}{ }^{-}$. For the carbene complexes the expected site for nucleophilic attack is the carbene carbon, not the methyl on OMe. This very interesting reaction is being pursued further. The studies to date have provided a much clearer understanding of the one- and two-electron processes in organometallic chemistry.

\section{Syracuse University Syracuse, NY 13244}

\section{Department of Chemical Engineering and Materials Science}

\section{The Relationship between Hydroxyl Groups on Oxide Surfaces and the Properties of Supported Metals Schwarz, J.A. 315-443-4575}

Supported metal catalysts are commonly prepared by depositing catalytic precursors from aqueous solutions of electrolytes onto high surface area oxides. A general conclusion of this lab's previous studies was that the performance of the finished catalyst depends on the characteristic properties of the hydroxyl inventory on the surface of the oxide support, both in wet and in (pseudo)dry conditions. Hydroxyl groups serve as adsorption or exchange sites during catalyst preparation. On the other hand, the configuration of hydroxyl groups still remaining on oxides after dehydration determines the acid-base characteristics of the catalyst, which is an important catalytic property. The purpose of the investigation is to characterize the relationship between the complex inventory of hydroxyl groups at oxide surfaces, the acid-base properties of oxides (both in aqueous solution and in the pseudo-dry state), and the resultant effects on the properties of catalytic materials formed by adsorption/ impregnation onto these hydroxylated supports during catalyst preparation. To this end the experimental and computational methods for measuring the Proton Affinity Distributions at the oxide/solution interfaces were developed. A common crystallographic model was used to describe the local configuration of hydroxyl groups on both the pseudo-dry oxide surface and the oxide/aqueous solution interface. This allows the extension of the concept of structurally determined intrinsic heterogeneity of pseudodry surfaces (as already known from the IR spectra of isolated surface hydroxyls) to the oxide/solution interface. The catalytic consequences of the acid sites' heterogeneity upon the impregnation step during catalyst preparation are being examined.

\section{Texas A \& M University College Station, TX $\mathbf{7 7 8 4 3}$}

\section{Department of Chemistry}

\section{Correlations between Surface Structure and Catalytic Activity/Selectivity Goodman, D.W. 409-845-0214

The project objective is to address those issues which are keys to understanding the relationship between surface structure and catalytic activity/selectivity. Of primary concern are those questions related to the origins of the enhanced catalytic properties of mixed-metal catalysts and the critical active site requirements for molecular synthesis and rearrangement. The experimental approach utilizes a microcatalytic reactor contiguous to a surface analysis system, an arrangement which allows in vacuo transfer of the catalyst from one chamber to the other. Surface techniques being used include Auger (AES), UV and X-ray photoemission spectroscopy (UPS and XPS), ion scattering spectroscopy (ISS), temperature programmed desorption (TPD), low energy electron diffraction (LEED), high resolution electron energy loss spectroscopy (HREELS), infrared reflection-absorption spectroscopy (IRAS), and scanning probe microscopies (STM/AFM). Currently the preparation, characterization, and determination of the catalytic properties of ultra-thin metal and metal oxide films are being explored. Specifically, the program is proceeding toward three goals: (1) the study of the unique catalytic properties of ultrathin metal films; (2) the investigation of the critical ensemble size requirements for principal catalytic reaction types; and (3) the modelling of supported catalysts using ultra-thin planar oxide surfaces.

\section{Solid-State NMR Studies of Zeolite Acidity Haw, J.F. 409-845-1966 \\ $\$ 136,731$}

High-Resolution ${ }^{1} \mathrm{H}$ nuclear magnetic resonance (NMR) will be used to characterize hydrogen bonding to the Bronsted acid site and H-D scrambling. The latter study may lead to a new way to characterize Bronsted acidity in 
zeolites. Other experiments will seek to thoroughly understand the ${ }^{1} \mathrm{H}$ NMR properties of HZSM-5 including the effects of chemical exchange and diffusion. The second strategy will use in situ ${ }^{13} \mathrm{C} N \mathrm{NMR}$ to look for intermediates in acid catalyzed reactions in zeolites. A laser heating system will be built for temperature jump and quench experiments that are expected to yield higher concentrations of reactive intermediates than are observed in standard variable temperature experiments. The focus of this project is Bronsted acidity in zeolites rather than reaction mechanisms per se.

\section{Catalysts and Mechanisms in Synthesis Reactions \\ Lunsford, J.H. $40.9-845-3455$ \\ $\$ 107,000$}

The objective of this research is to understand the role of surface-generated gas-phase radicals in the catalytic oxidation of hydrocarbons, with emphasis on the conversion of methane to more useful chemicals and fuels. Matrix isolation electron spin resonance (MIESR), variable ionization mass spectrometry (VIMS) and laser-induced fluorescence (LIF) methods have been used to detect radicals that emanate from or react with metal oxide surfaces during a catalytic reaction. The detection of methyl radicals using the MIESR systems has been effective in establishing the mechanism by which methane is converted to ethane and ethylene. Most recently the technique has been used to demonstrate that methyl and allyl radicals are involved in the catalytic cross coupling of methane and propylene to form butene. The VIMS system has been used to follow the reaction of methyl radicals with nitric oxide to produce nitrosomethane, which is believed to be an intermediate in the reduction of nitric oxide by methane. It has been demonstrated that water and oxygen react oyer strongly basic oxides, such as lanthanum oxide, to form hydroxyl radicals in the temperature range 1200 to $1350 \mathrm{~K}$. The hydroxyl radicals are believed to be formed by the abstraction of hydrogen atoms from water at reactive surface oxygen ions, and they may play an important role in catalytic combustion.

\section{University of Texas at Austin Austin, TX 78712}

\section{Department of Chemical Engineering}

\section{Catalytic Hydrocarbon Reactions over Supported Metal Oxides \\ Ekerdt, J.G. 512-471-4689

The primary goal of this research program is to determine how catalyst composition, redox ability, structure, and neighboring sites control the catalytic properties of metal oxides. Molybdenum, tungsten, chromium, and vanadium cations are supported on silica using preparation methods that enable molecular control over the structure and oxidation state. Supported structures featuring crystallites, isolated cations, and dimers with either a metal-metal or bridging sulfur bond are used as model templates to investigate key steps in catalytic oxidation and hydrogenation catalysis. Key steps in oxidation catalysis include $\mathrm{C}-\mathrm{H}$ abstraction and reduction of the cation site, and these are studied using oxidative dehydrogenation of butenes to butadiene and 1-butene isomerization to cisand trans-2-butene. Key steps in hydrogenation catalysis include adsorption and selective hydrogenolysis of the C-heteroatom bond, and these are studied during the hydrogenation of thiophenes and thiols. The research effort focuses on reaction studies over the different structures. When necessary the appropriate spectroscopic methods are used to characterize the cation structure and oxidation state.

\section{Department of Chemistry}

\section{Morphological Aspects of Surface Reactions \\ White, J.M. 512-471-3704}

Our work examines substrate morphology and fragments synthesized on surfaces using thermal, electron, and photon activation techniques. Our ongoing investigations into the surface chemistry of halides continued with a study of chloroiodomethane coadsorbed with hydrogen and oxygen on the (111) surface of platinum. At or below $100 \mathrm{~K}$, $\mathrm{ClCH}_{2} \mathrm{I}$ adsorbs molecularly on clean, D-covered and $\mathrm{O}$ covered surfaces. Some $\mathrm{ClCH}_{2} \mathrm{I}$ in the first monolayer dissociates during heating. In the absence of $\mathrm{D}$ and $\mathrm{O}$, dissociation begins with $\mathrm{C}-\mathrm{I}$ bond cleavage at $\sim 150 \mathrm{~K}$, and several competitive reactions ensue. Coadsorbed $\mathrm{D}$ atoms weaken the bonding between $\mathrm{ClCH}_{2} \mathrm{I}$ and the surface, decrease $\mathrm{ClCH}_{2} \mathrm{I}$ dissociation, and suppress the complete decomposition to carbon for dissociating $\mathrm{ClCH}_{2} \mathrm{I}$ molecules. In temperature programmed desorption with coadsorbed $D$, we see $H-D$ exchange products for methane but not for methyl chloride. Coadsorbed $O$ atoms slightly attenuate $\mathrm{ClCH}_{2} \mathrm{I}$ dissociation but strengthen its surface bonding. In addition to this work, we prepared a thorough review of research on the preparation and kinetic characterization of hydrocarbon fragments, primarily phenyl, vinyl, and methyl groups, on transition metals using nonthermal activation methods.

\section{Tulane University New Orleans, LA 70118}

\section{Department of Chemical Engineering}

\section{The Formation of Silica, Alumina, and Zirconia: Supported High Surface Area Monometallic and Bimetallic Catalysts Gonzalez, R.D. 504-865-5772

This study focuses on the preparation of thermally stable metal catalysts. The main thrust of this research is to improve the stability of supported metal catalysts in both an oxidizing and a reducing atmosphere. Theoretical studies suggest that when a match between the average pore size of the support and the particle size of the supported metal is achieved, the resulting catalysts should resist sintering as a result of surface diffusion. In this laboratory particle and pore diameters for $\mathrm{Pt} / \mathrm{SiO}_{2}$ catalysts prepared by the sol-gel method have been successfully matched. Thermally stable $\mathrm{Pd} / \mathrm{SiO}_{2}$ catalysts have also been prepared using the sol-gel method. However, the resulting catalysts are not stable in $\mathrm{H}_{2}$ at temperatures in excess of $650^{\circ} \mathrm{C}$. The 
conclusion from this study is that in the presence of flowing $\mathrm{H}_{2}$, an unstable $\mathrm{Pd} \beta$-hydride is formed. In order to inhibit the formation of the $\mathrm{Pd} \beta$-hydride, it is proposed to prepare Pd bimetallic clusters by alloying Pd with a second metal. Candidates include Pt and Rh. The presence of bimetallic clusters that incorporate dual metal sites is determined using EDXS (Energy Dispersive X-Ray Spectroscopy). However, the formation of a bimetallic cluster does not guarantee the inhibition of $\beta$-hydride formation. Conditions that favor the formation of the hydride can be determined using differential scanning calorimetry. Preparative techniques that inhibit its formation can then be incorporated into the preparative sequence. A second project involves the preparation of superacid zirconia catalysts using the sol-gel synthesis method. It is hoped that in the near future unfriendly liquid acids such as $\mathrm{H}_{2} \mathrm{SO}_{4}$ and $\mathrm{HF}$ can be replaced by solid acids in processes such as hydrocarbon isomerization and alkylation. Sulfonated zirconia, although a very active low temperature isomerization catalyst, deactivates very rapidly. This deactivation is related to the rapid disappearance of active acid isomerization sites and the formation of surface carbon. The acid sites responsible for rapid deactivation are in all likelihood due to strong Bronsted sites. Several solutions to this rapid deactivation are possible. In order to decrease the rate of carbon formation, a hydride donor such as methylcyclopentane or adamantane can be added to the feed. The addition of these hydride donors may result in the termination of the long-lived tertiary carbonium ions prior to $\mathrm{C}-\mathrm{C}$ bond cleavage. If cleavage does occur, the addition of a good hydrogenation catalyst such as $\mathrm{Pt}$ may result in less carbon being formed.

\section{University of Utah Salt Lake City, UT 84112}

\section{Department of Chemistry}

\section{Ligand Intermediates in Metal-Catalyzed Reactions \\ Gladysz, J.A. \\ $801-581-4300$

The first goal of this project is the synthesis, isolation, and characterization of homogeneous transition metal complexes containing ligand types $\left(-\mathrm{CHO},-\mathrm{CHOH},-\mathrm{CH}_{2} \mathrm{OH}\right.$, $-\mathrm{C},=\mathrm{CH}_{2}, \mathrm{H}_{2} \mathrm{C}=\mathrm{O},-\mathrm{OCHO},-\mathrm{OCH}_{2} \mathrm{R}, \mathrm{CO}_{2}$, etc.) intermediate in $\mathrm{C}_{1} / \mathrm{C}_{2}$ catalytic reactions. The second goal entails the characterization of ligand intermediates in other important feedstock conversions, and the identification of new types of binding modes and bond activation processes. Mechanistic understanding of key steps and insight for the design of new catalysts is sought. The following topics are under active investigation: (1) the determination of relative ligand binding affinities towards Lewis acidic metal centers, including divergent kinetic and thermodynamic $\mathrm{O}=\mathrm{C} / \mathrm{C}=\mathrm{C}$ selectivities in bifunctional nonconjugated substrates; (2) the characterization of nonclassical metal $\mathrm{C}-\mathrm{H}$ "sigma bond" complexes as reaction intermediates; (3) unusual base-induced vinylic $\mathrm{C}-\mathrm{H}$ bond activation reactions of alkene ligands; (4) the synthesis, structure, electronic properties, and reactivity of complexes that contain unsupported and supported $\mathrm{C}_{2}$ and $\mathrm{C}_{3}$ linkages spanning two metals; (5) $\mathrm{C}-\mathrm{H}$ bond activation reactions of aromatic nitrogen heterocycles such as pyrrole, and new HON processes; and (6) the synthesis, structure, and reactivity of $\mathrm{CO}_{2}$ complexes in coupled redox states.

\section{Carbon-13 NMR of Solid-State Hydrocarbons and Related Substances} Grant, D.M.; Pugmire, R.J. 801-581-8854

$\$ 118,000$

The project objective is to develop new nuclear magnetic resonance (NMR) techniques to study solid organic materials applied to gain structural and chemical information on model compounds and natural samples. The most important achievements have been (1) developing new spatial correlation techniques to measure ${ }^{13} \mathrm{C}$ chemical shielding tensors in single crystals; (2) improving theoretical methods for the calculation of shielding tensors; (3) developing a new magic angle slow turning (MAT) method of obtaining two-dimensional solid-state NMR spectra where the isotropic shift is projected along one axis and tensor powder patterns along the second axis; and (4) applying these ${ }^{13} \mathrm{C}$ shielding tensor methods in the characterization of coals of all ranks. The single-crystal correlation techniques characterize the chemical shift tensor and its orientation in the molecular frame. The accuracy of the single crystal methods is sufficiently high that it may be used along with quantum mechanical methods to refine crystal structures of fused aromatic hydrocarbons. The various NMR spectroscopic methods provide carbon-13 NMR shielding tensors for a variety of polycyclic aromatic systems and hydroaromatics such as acenapthalene, perylene, triphenylene, and several naphthalene derivatives. Emphasis is on structural details in these hydroaromatics because of their importance in fossil fuels. Theoretical calculations have been extended to these model compounds to explain and support the experimental conclusions. Spectroscopic analyses of non-protonated to protonated aromatic carbons agree with elemental analyses and dipolar dephasing NMR techniques. The new two-dimensional methods are useful for analyzing the structure of coals of various ranks; the fraction of condensed carbons obtained may be used to estimate average cluster size in condensed polyaromatic hydrocarbons, and these data have provided key parameters for use in coal devolatilization theories being developed in concert with work in the advanced combustion research institute.

\section{Virginia Polytechnic Institute and State University Blacksburg, VA 24061}

\section{Department of Chemical Engineering}

\section{Influence of Surface Defects and Local Structure on Acid/Base Properties and Oxidation Pathways over Metal-Oxide Surface \\ Cox, D.F. \\ 703-231-6829

The purpose of the project is to examine the effect of surface defects (primarily oxygen vacancies) and local structure on catalytic oxidation reactions over metal oxide materials. The $\mathrm{SnO}_{2}(110)$ surface is being investigated because of the flexibility allowed in controlling surface cation coordination numbers, oxidation states, and the 
selective introduction of two different types of surface oxygen vacancies. The effect of surface properties on the reaction pathways of oxygenates is being examined. Previous results have shown that the dissociation of Bronsted acids (formic acid, methanol, water) can be alternately controlled by either the acidity of the molecules or the surface condition. Weaker acids dissociate more readily in the presence of cations with a reduced coordination relative to the stoichiometric surface, i.e., the nature of the surface cations (acid sites) controls the activity. However, this effect is limited to a specific range of surface compositions. The most highly defective surface exhibits the lowest probability for dissociate adsorption. The relative acidity/ basicity of the two specific surface defects are currently being tested with probe reactions. New directions include the study of the effects of surface condition on the selective oxidation of propene, the characterization of point defects with STM, and ab initio density functional calculations of defect geometry and electronic structure.

\section{University of Virginia Charlottesville, VA 22903}

\section{Department of Chemical Engineering}

\section{Structure-Property Relationships for Binary Metal Oxide Catalysts \\ Davis, $R$. J. 804-924-6284}

Solid oxide surfaces often expose low-coordinated atoms that can function as active sites in catalytic reactions. In this work, the roles of local structure and second shell composition on the activity of binary mixed metal oxides that are useful for base catalysis will be studied. Initially, alkali metal-silicon mixed oxides will be studied as model catalysts. Determination of local structure and composition of these oxide catalysts requires the use of a wide variety of techniques including $\mathrm{X}$-ray absorption spectroscopy, infrared spectroscopy, and temperatureprogrammed desorption of probe molecules. One of the objectives of this project is to develop additional experimental methods that can be used to analyze the structure of basic, mixed oxide surfaces. The reactivity of these model alkali metal oxides for several reactions of importance for fuels production, chemicals production, and overall energy utilization will also be examined. Results from these studies will allow the evaluation of the effects of atomic-level mixing on the reactivity of oxide components and the proposal of new structure-property relationships for heterogeneous base catalysis.

\section{University of Washington Seattle, WA 98195}

\section{Department of Chemistry}

\section{Model Oxide-Supported Metal Catalysts for Energy Technologie Campbell, C.T. 206-543-3287

This project will identify the active form of surface $\mathrm{Cu}$ in the methanol synthesis reaction over $\mathrm{Cu} / \mathrm{ZnO}$ catalysts and elucidate the influence of $\mathrm{ZnO}$ on the morphological and chemical properties of supported $\mathrm{Cu}$ particles. The kinetics of methanol synthesis over vapor-deposited $\mathrm{Cu}$ thin films on $\mathrm{ZnO}$ single-crystal faces are compared with those over industrial $\mathrm{Cu} / \mathrm{ZnO}$ catalysts. The growth kinetics, structure, annealing behavior, and chemisorption properties of thin $\mathrm{Cu}$ films on several $\mathrm{ZnO}$ single-crystal faces are also being studied using surface spectroscopies. Other late transition metals on other oxides are also being studied. The goal is to clarify the geometric, dynamic, and energetic factors that control the microstructure of the metal/oxide interface, and to rationalize the interplay between this microstructure and chemical reactivity. Copper can form islands on the oxygen-terminated $\mathrm{ZnO}(0001)$ face that are only one atom thick, and these behave chemically much like $\mathrm{Cu}(110)$, which is one of the most catalytically active bulk $\mathrm{Cu}$ faces. One goal is to see how the substrate geometry influences the morphology, stability, and chemical properties of such thin $\mathrm{Cu}$ particles. This $\mathrm{Cu} / \mathrm{ZnO}$ system serves an important role as a prototype for metal oxide catalysts where the choice of support is critical.

\section{Homolytic Activation of Hydrocarbons and Hydrogen by Persistent Metal Radicals \\ Heinekey, $M$. 206-543-7522}

$\$ 93,077$

Preparation of highly reactive, coordinatively unsaturated rhenium cations of the form $\left[\operatorname{Re}(\mathrm{CO})_{3}\left(\mathrm{PR}_{3}\right)_{2}\right]^{+}(\mathrm{R}=$ cyclohexyl, isopropyl, phenyl) has been carried out by protonation of the corresponding neutral methyl complexes with $\mathrm{H}\left(\mathrm{Et}_{2} \mathrm{O}\right)_{2} \mathrm{~B}\left(\mathrm{Ar}^{\prime}\right)_{4}\left(\mathrm{Ar}^{\prime}=3,5-\left(\mathrm{F}_{3} \mathrm{C}\right)_{2} \mathrm{Cx}_{6} \mathrm{H}_{3}\right)$. An X-ray study of the $\mathrm{PCy}_{3}$ complex reveals that the coordinative unsaturation of the formally 16 electron complex is relieved by coordination of one of the C-H bonds of the cyclohexyl group to the metal in an agostic interaction. The cationic complexes can be reduced by one electron using sodium metal to form the corresponding 17 electron neutral radical species. This reaction represents the first practical preparation of these radical species. The 16 electron cations are very electrophilic and react with a number of small molecules including $\mathrm{H}_{2}$ and $\mathrm{N}_{2}$. The resulting $\mathrm{H}_{2}$ complexes are isolable and have properties comparable to the neutral tungsten analogs. Rapid exchange of coordinated $\mathrm{H}_{2}$ for external $\mathrm{D}_{2}$ has been observed, in addition to fast atom exchange to afford HD. The mechanism of this novel reaction is being investigated. In related investigations of highly electrophilic cations, we have found that rhenocene cation $\left(\mathrm{Cp}_{2} \mathrm{Re}^{+}\right)$ can be prepared by reductive elimination of methane from the corresponding methyl hydride complex. Rhenocene reacts rapidly with methylene chloride and the $\mathrm{C}-\mathrm{Cl}$ bond of freons to give alkyl chlorides. 


\section{University of Wisconsin at Madison Madison, WI 53706}

\section{Department of Chemical Engineering}

\section{Thermodynamic and Kinetic Aspects of Surface Acidity \\ Dumesic, J.A. 608-262-1092 \\ $\$ 110,000$}

This project involves the characterization of catalyst acidity and basicity, using microcalorimetric measurements, infrared spectroscopic studies, ${ }^{2} \mathrm{D}$ NMR studies of Bronsted acid/base sites, and reaction kinetics studies of acid-catalyzed reactions. For example, we have investigated the acid/base properties of $\gamma-\mathrm{Al}_{2} \mathrm{O}_{3}$ following addition of $\mathrm{K}_{2} \mathrm{O}, \mathrm{MgO}, \mathrm{La}_{2} \mathrm{O}_{3}, \mathrm{Eu}_{2} \mathrm{O}_{3}, \mathrm{SnO}$, and $\mathrm{SnO}_{2}$. We have studied the catalytic cracking of isobutane and 2methyl-hexane over USY-based catalysts. Most recently, we have studied the isomerization of n-butane at $423 \mathrm{~K}$ over sulfated-zirconia catalysts. These catalysts contain strong Lewis and Bronsted acid sites, having differential heats of $\mathrm{NH}_{3}$ adsorption in the range of $130-165 \mathrm{~kJ} / \mathrm{mol}$. These acid sites are responsible for the longer-term activity of the catalyst, and they deactivate more gradually under reaction conditions. Acid sites that adsorb ammonia more weakly than $120 \mathrm{~kJ} / \mathrm{mol}$ are not active for n-butane isomerization at $423 \mathrm{~K}$.

\section{Department of Chemistry}

\section{Organometallic Chemistry of Bimetallic Compounds \\ Casey, C.P. \\ 608-262-0584

Four different projects at the interface between organometallic chemistry and homogeneous catalysis are being pursued. All are designed to give increased understanding of the mechanisms of organometallic chemistry related to homogeneous catalysis. (1) Bimetallic catalysis has almost unlimited potential but very few systems are known in which there is direct evidence for involvement of a bimetallic compound. The hydrogenation of alkynes by $\mathrm{Cp}(\mathrm{CO})_{2} \operatorname{Re}(\mu-\mathrm{H}) \mathrm{Pt}(\mathrm{H})\left(\mathrm{PPh}_{3}\right)_{2}$ to give rhenium-alkene complexes suggests that it may be possible to develop a catalytic system based on more labile metal-alkene complexes. The use of indenyl systems and Mn-based systems will be investigated. (2) Hydroformylation with chelating diphosphines with wide natural bite angles near $120^{\circ}$ gives high regioselectivity for straight chain aldehydes. Attempts to explain this selectivity with steric effects have failed and the role of electronic effects of chelating ligands will be investigated. (3) $\mathrm{Cp}^{*}(\mathrm{CO})_{2} \operatorname{Re}=\operatorname{Re}(\mathrm{CO})_{2} \mathrm{Cp}^{*}$ is a highly unusual and reactive dimer of a $d^{6}-16 \mathrm{e}$ fragment. The very facile reversible addition of $\mathrm{H}_{2}$, the very facile addition of ligands, and the diverse reaction of alkynes with this fascinating compound will be investigated. (4) The reaction of $\mathrm{Cp}_{3}{ }_{3} \mathrm{Co}_{3}\left(\mu_{2}-\mathrm{H}\right)_{3}\left(\mu_{3}-\mathrm{H}\right)$ with acetylene to produce the bis ethylidyne cluster $\mathrm{Cp}_{3}{ }_{3} \mathrm{Co}_{3}\left(\mu_{3}-\mathrm{CCH}_{3}\right)_{2}$ will be investigated in detail with isotopically labeled materials. This reaction is interesting in relation to transformations on metal surfaces.
356. Mechanism and Design in Homogeneous Catalysis

Landis, C.R.

608-262-0362

$\$ 102,000$

This project is a broad-based program aimed at designing highly selective organotransition metal catalysts and at elucidating fundamental mechanisms of selectivity control in metal catalyzed reactions. This work is guided by the following assertions: (1) computer modeling methods combined with existing data on catalyst structure and selectivity is a promising new approach to the problem of designing catalysts for high selectivity (2) a new level of sophistication in catalyst design, discrimination via attractive secondary interactions (vide infra) rather than steric repulsions, should be pursued vigorously as a means of regio- and stereo-selectivity control in homogeneous catalysis and (3) of equal importance to creating highly selective catalysts is understanding, at the molecular level, how selectivity is achieved. This work is illustrated primarily by application to selectivity control in catalytic hydrogenation, hydroformylation, hydrocarboxylation, and hydrocyanation processes but the methods that are being developed are quite general.

\section{University of Wisconsin at Milwaukee Milwaukee, WI 53201}

\section{Department of Chemistry}

\section{Aluminum Coordination and Active Catalytic Sites in Aluminas, Y Zeolites, and Pillared Clays \\ Fripiat, J. \\ 414-229-5852 \\ $\$ 93,000$}

In order to achieve a more refined knowledge of the surface topology of the acid sites determined earlier from the application of ${ }^{27} \mathrm{Al}$ cross polarization technique from the protons of chemisorbed ammonia, our efforts have been directed toward the use of REDOR (rotational-echo double-resonance). A comparative examination of the REDOR responses with one pulse and cross-polarization excitation results in obtaining structural information concerning the surroundings of four, five, and six coordinated $\mathrm{Al}$ sites and discriminating between surface, intermediate, and bulk species in aluminas. Now this study is extended to silicon and aluminum in zeolites. The measurements of the numbers of Bronsted and Lewis acid sites by CO FTIR TPD have been extended to dealuminated HZSM5 (small number of Lewis sites) and dealuminated HY (large number) and the characteristics of the pentane isomerization and cracking reactions are being compared on both catalysts. The modification of zeolite micropore volume resulting from thermal dealumination have been examined by measuring the changes in the unit cell parameters as well as the $\mathrm{N}_{2}$ and $n$-pentane adsorption isotherms. For cubic faujasite and orthorhombic HZSM5, the differential decrease of the micropore volume per nonframework aluminum (NFAl) can be predicted by assuming that NFAl is concentrated in the pores available to $\mathrm{N}_{2}$. For orthorhombic $\mathrm{H}$-mordenite, NFAl is homogeneously spread in all pores, available or not to $\mathrm{N}_{2}$. The Gurvitch rule is respected in HY and HZSM5, but not in mordenite. 


\section{An Investigation of Molybdenum and Molybdenum Oxide Catalyzed Hydrocarbon Formation Reactions} Tysoe, W.T.

414-229-5222

Model oxide olefin metathesis catalysts consisting of $\mathrm{MoO}_{2}, \mathrm{MoO}_{3}$, metallic molybdenum, and oxygen overlayers on molybdenum, were characterized using various surface-sensitive techniques including X-ray and ultraviolet photoelectron spectroscopies and low-energy electron diffraction and were tested using an isolatable high pressure reactor. It was found that the reactivity order at high-reaction temperatures ( $>650 \mathrm{~K})$ was $\mathrm{MoO}_{2}>\mathrm{MoO}_{3}>$ metallic Mo. Measurement of the temperature dependence of the reaction using $\mathrm{MoO}_{2}$ revealed two different activation energies. Below $\sim 650 \mathrm{~K}$ the reaction activation energy was $\sim 6 \mathrm{kcal} / \mathrm{mol}$; a value corresponding to that found experimentally for alumina-supported molybdenum oxide. In addition, absolute reaction rates for oxide catalysts at low temperature were very close to supported catalysts with high oxide loadings. The activation energy above $\sim 650 \mathrm{~K}$ was substantially higher at $\sim 60$ $\mathrm{kcal} / \mathrm{mol}$. The metathesis activity was also found to be a function of oxygen coverage when using catalysts consisting of oxygen overlayers 'on molybdenum. The hightemperature activity was further studied by reacting ethylene over metallic molybdenum, which led to the formation of higher hydrocarbons with a products distribution which was well described by a Shultz-Flory plot suggesting their synthesis via a surface $C_{1}$ polymerization pathway. Ultraviolet photoelectron spectroscopic measurements for alkene adsorption on oxygen-covered molybdenum indicate that the alkene dissociatively adsorbs on these surfaces. Temperature-programmed desorption experiments using isotopically labelled ${ }^{13} \mathrm{C}^{12} \mathrm{CH}_{4}$ show isotope scrambling, suggesting that surface species can indeed recombine to form ethylene in accord with the catalytic results describe above. Similar photoelectron spectroscopic experiments reveal that $\mathrm{CH}_{2} \mathrm{I}_{2}$ decomposes on these surfaces to form adsorbed $\mathrm{C}_{1}$ species. This chemistry is being investigated further to probe carbene recombination. Finally, analysis of the catalyst after reaction shows that metathesis proceeds in the presence of a carbonaceous layer. A similar effect is found in palladiumcatalyzed benzene formation from acetylene. The role of this carbonaceous layer in the catalytic reaction is being investigated.

\section{Yale University New Haven, CT 06520}

\section{Department of Chemical Engineering}

\section{Acidity and Effect of Acidity on Supported Metals \\ Haller, G.L. 203-432-4378 \\ $\$ 119,000$}

A new class of silica-alumina mesoporous molecular sieves is under investigation. These materials have very narrow and variable pore size distributions, variable silical alumina ratios, and are pseudo-crystalline (diffract $\mathrm{X}$ rays, but lack three-dimensional crystallinity). These materials are solid acids that may have acid sites with strength between that of amorphous and crystalline (zeolite) silica-aluminas. The structure of these materials will be studied with solid state NMR to determine their acid strength quantitatively and to use them as reference materials to test hypotheses about the structure/acidity correlations of amorphous silica-aluminas of variable composition and acidity. Amorphous, mesoporous/pseudocrystalline, and crystalline (zeolite) silica-aluminas will be used as supports for $\mathrm{Pt}$ and $\mathrm{Pd}$ particles and the relative effect of the acidity of the supports on the activity and selectivity of several probe catalytic reactions will be determined. The structure and interaction of the metal particles with the acid sites will be studied by X-ray absorption. Pt in mesoporous silica-alumina will be explicitly compared to $\mathrm{Pt} / \mathrm{L}$-zeolite (using the same $\mathrm{Si} / \mathrm{Al}$ ratio as for the zeolite) for $\mathrm{n}$-hexane aromatization with the objective of determining the role of pore size on activity and selectivity in parallel channels.

\section{Department of Chemistry}

\section{Catalytic Oxidation of Hydrocarbons by Binuclear Fe Complexes \\ Caradonna, J.P. 203-432-5221

This project is investigating the ability of non-heme iron metalloenzyme reactivity models to catalyze the oxidation of alkane and arene molecules, including the conversion of methane and ethane to methanol and ethanol, respectively. The objective of this project is to characterize the electronic structure and reactivity properties of a series of non-heme mononuclear and dinuclear iron complexes, characterize any intermediates formed during oxygen atom transfer chemistry, and elucidate the mechanisms and specificity of the reactions. Comparisons to analogous heme-based chemistry will be made. A series of diferrous, ferric/ferrous, and diferric complexes were synthesized from simple polyamide and polycarboxylate ligands and spectroscopically characterized. The diferrous compounds, which are all powerful reductants, heterolytically decompose peracids and are capable of acting as oxygen atom transfer catalysts when reacted with oxygen atom donor compounds and simple olefins, yielding both allylic oxidation and epoxidation products. An isostructural dicobalt(II) compound gives rise to only epoxidation products. Intermediates observed during catalytic turnover and reactions with dioxygen are currently being examined.

\section{Alkane Photoreactions with Mercury Vapor Crabtree, R.H. 203-432-3925}

$\$ 95,000$

In the latest period, the $\mathrm{Hg}$ photosensitized work has moved very rapidly. $\mathrm{Hg}^{*} / \mathrm{NH}_{3}$ has proved to react with a variety of saturated fluorocarbons including $\mathrm{CF}_{4}$. The products are a variety of amine and imine species, some of which have been fully characterized. A collaboration with Prof. PEM Siegbahn (Stockholm) has shown that $\mathrm{H}+\mathrm{CF}_{4}$ has a high barrier $(20 \mathrm{kcal} / \mathrm{mol})$ accounting for the inertness of fluorocarbons under $\mathrm{Hg}^{*} / \mathrm{H}_{2}$ conditions. A variety of clues suggest that the Rydberg molecule $\mathrm{NH}_{4}$ is the true reactive species in $\mathrm{Hg}^{*} / \mathrm{NH}_{3}$ experiments. Siegbahn finds a much lower barrier (not yet quantified) for $\mathrm{NH}_{4}+$ $\mathrm{CF}_{4}$. This we ascribe to the possibility of proton coupled electron transfer in this case: electron transfer from $\mathrm{NH}_{4}$ 
to $\mathrm{CF}_{4}$, accompanied by proton transfer within the resulting incipient $\mathrm{NH}_{4}{ }^{+} / \mathrm{CF}_{4}-$ ion pair. In related work, reactions of $\mathrm{Hg}^{*}$ with alkylarenes has been shown to give C-C bond cleavage in the alkyl side chain, a very unexpected result. Theoretical work (Siegbahn) suggests that an intermediate ( $\eta^{2}$-arene)Hg exciplex is involved in an energy transfer from $\mathrm{Hg}^{*}$ to the arene and that C-C cleavage occurs in the triplet arene so formed.

\section{Separations and Analysis}

\section{University of Arizona Tucson, AZ 85721}

\section{Department of Chemistry}

\section{Raman Spectroscopy of Reversed-Phase Liquid Chromatographic Alkylsilane Stationary Phases on Thin Silica Films Pemberton, J.E. $520-621-8245$

Reversed-phase liquid chromatography (RPLC) is the most popular mode of liquid chromatography used today. The stationary phase used for RPLC typically consists of silica gel onto which an alkylsilane layer has been covalently bound. Despite intense effort, a fundamental understanding of the molecular basis of the separation process has not been achieved. In a new approach to the Raman spectroscopy characterization of RPLC interfaces, we are using a novel layered structure culminating with an outer layer of a thin silica film formed by spin coating a sol-gel solution. The sol-gel approach to the synthesis of these silica films is central to the proposed effort in that control over the microstructure of the silica layer can be achieved to make silica gel systems that are chromatographically relevant. Studies are underway to further develop and refine these sol-gel based silica thin films, to improve the surface Raman spectroscopy to enable routine characterization of covalently-bonded alkylsilane layers at chromatographically-relevant surface coverages, and to investigate the influence and interactions of mobile phase solvents and solutes on the structure, orientation, and order of alkylsilane bonded phases. This work should enable the first systematic Raman spectroscopic characterization of covalently bonded alkylsilane stationary phase behavior at silica surfaces in environments relevant to true RPLC systems to be performed thus furthering the understanding of the molecular basis for retention in RPLC systems.

\section{Auburn University Auburn, AL 36849}

\section{Department of Chemical Engineering}

\section{Interfacial Chemistry in Solvent Extraction Systems \\ Neuman, R.D. 344-844-2017}

The microscopic interfaces, i.e., association microstructures, in acidic organophosphorus extraction systems associated with $\mathrm{Na}^{+}, \mathrm{Co}^{2+}, \mathrm{Ni}^{2+}, \mathrm{Zn}^{2+}$, and $\mathrm{UO}_{2}{ }^{2+}$ are being systematically investigated using various physicochemical, spectroscopic and scattering techniques in order to resolve the physicochemical nature and structure of reversed micelles of metal-extractant complexes, the thermodynamics of the formation and growth of reversed micelles, the effect of organic phase additives on the structure of reversed micelles, and the relationship between reversed micellar structure and selectivity. A new structural model-the "open water-channel" model-for reversed micelles and a quantitative model that relates the extractability and selectivity to the size of reversed micelles in solvent extraction systems have been proposed and are being experimentally verified. Advanced laser techniques (SLLS, LIF, FRAP) are being utilized to characterize the properties, structure, and dynamics of extractant-laden liquid-liquid interfaces. A study of the permeation of metal-extractant species, in particular, association microstructures, is being initiated using a state-of-the-art surface force apparatus in order to examine interfacial/transport phenomena within supported liquid membranes (SLMs). This innovative research program will make significant contributions to the science and technology of liquid-liquid extraction and supported liquid membranes.

\section{Brigham Young University Provo, UT 84602}

\section{Department of Chemistry}

\section{Novel Macrocyclic Carriers for Proton-Coupled Liquid Membrane Transport \\ Lamb, J.D.; Bradshaw, J.S.; Shirts, R.B.; Izatt, R.M. 801-378-3145}

The metal cation selectivity of macrocyclic ligands such as crown ethers and cryptands is applied to making cation separations in hydrophobic liquid membranes and other systems. Potential macrocyclic ligand carriers are designed and synthesized, then screened for cation binding characteristics using potentiometric titration, solvent extraction, calorimetry, NMR, and X-ray crystal structure determinations. Macrocycles which demonstrate potential for separations are then incorporated into liquid membrane systems. Focus is placed on the synthesis and characterization of new proton ionizable macrocycles that permit coupling of cation transport to the reverse flux of protons. Macrocycles containing acidic moieties within the macrocyclic ring structure are particularly emphasized. State-of-the-art molecular mechanics modeling techniques are used in the design phase of the project. Current focus is being given to a new type of polymer inclusion membrane for stable, long-term separations. A new, hightemperature calorimeter is also being used to study the binding of macrocycles to metal cations at high temperatures. 
Brown University

Providence, RI 02912

\section{Department of Chemistry}

\section{Thermal Generation of the Photoacoustic Effect \\ Diebold, G.J. 401-863-3586 \\ $\$ 86,000$}

Research during this period is directed toward determining the properties of suspensions of particles using photoacoustic techniques. In particular, two problems are being addressed. First, experiments with reverse micelles are being conducted to see if thermal properties can be determined from transient grating signals. In the case of reverse micelles, a chromophore is placed inside the micelle so that heat is deposited by the laser into the interior of the micelle only. Since water has a small thermal expansion coefficient, it is only after heat diffuses into the surrounding organic liquid that a photoacoustic effect is generated. The rate of heat diffusion from the micelle thus determines the time response of the transient grating. Experiments are directed toward determining whether laboratory data can be analyzed to determine properties of micelles. Second, experiments are being carried out to determine the origin of the second harmonic component of the diffracted light signal found in solutions of carbon particles. However, development of a theory is challenging because of the difficulty in describing the overall time development of the fluid density when the driving force for the motion arises from effects that take place at individual sources spread throughout the fluid.

\section{Colorado School of Mines Golden, CO 80401}

\section{Department of Chemical Engineering}

\section{A Mechanistic Study of Molecular Sieving Inorganic Membranes for Gas Separation Way, J.D. 303-273-3519 $\$ 87,000$}

The objectives of this research are to investigate selective transport mechanisms in microporous, silica membranes and to examine the effects of membrane microstructure and surface chemistry on separation performance. A further objective is to use theoretical chemistry to simulate the adsorption and transport of penetrants in pores of molecular dimensions. Quantum mechanics simulations were used to estimate the heats of adsorption of gases on model surfaces such as silanol $\left(\mathrm{H}_{3} \mathrm{SiOH}\right)$ and silicic acid $\left(\mathrm{Si}(\mathrm{OH})_{4}\right)$. Very high mixed gas separation factors of 11.5 for $\mathrm{O}_{2} / \mathrm{N}_{2}$ and 250 for $\mathrm{CO}_{2} / \mathrm{CH}_{4}$ have been measured at $298 \mathrm{~K}$ and feed gas pressures of $21.7 \mathrm{bar}$. These unusually high separation factors are attributed to surface interactions between the penetrant molecules and the silica surface. Therefore, the surface chemistry and its role on the adsorption and transport of gases will be independently studied using FT-IR and NMR spectroscopy. These analytical techniques will be used to determine the relative populations of polar hydroxyl groups and nonpolar siloxane species on the membrane surface as a function of temperature and pressure. Measurements with mixtures containing propane indicate a capillary condensation mechanism in which the propane blocks the transport of $\mathrm{N}_{2}$ and $\mathrm{He}$.

\section{Colorado State University Fort Collins, CO 80523}

\section{Department of Chemistry}

\section{A Multinuclear Magnetic Resonance Study of the Interactions of Pollutants with Major Soil Components \\ Maciel, G.E. 970-491-6480 \\ $\$ 120,000$}

This project will provide detailed information on the fundamental chemical-physical behavior (including chemical transformations) of certain organic contaminants with major soil constituent; specifically clays and humic materials. Chemical interactions and physical behavior will be studied via state-of-the-art nuclear magnetic resonance (NMR) experiments on solid and aqueous-solution samples. The specific tasks of this project are (1) to generate, or update and refine, the chemical-structural and NMR data bases of the specific clays and humics chosen for study; (2) elucidation of the behaviors of individual pollutants with these specific humics and clays; (3) study of the fundamental interactions in clay-humic "complexes"; (4) elucidation of pollutant mobility and diffusion in the specific humic and clay systems; (5) chemical structural characterization of ternary systems (pollutant-humicclay); and (6) exploratory studies of pollutant/whole-soil systems. Results of this kind of study are needed in the long term for development of reliable and versatile soilgroundwater models of pollution.

\section{University of Delaware Newark, DE 19716}

\section{Department of Chemistry and Biochemistry}

\section{Linear and Nonlinear Spectroscopic Probing of Solute Interactions with Chemically Modified Silica Surface Wirth, M.J. 302-831-6771

The work focuses on understanding physically how organic bases are retained chromatographically, which is essential to the improvement of protein and DNA separations. The separation of these biomolecules is crucial to the investigation of health effects of energy-related compounds. Fluorescence spectroscopy is being used to probe the orientation and rotational diffusion of adsorbates on chemically modified silica surfaces to provide a fundamental understanding of chromatographic separation. The new technique of near-field scanning optical microscopy is being explored as a means of providing $100 \mathrm{x}$ higher surface selectivity than has previously been possible with evanescent wave fluorescence spectroscopy. This advance potentially allows fluorescence studies of dilute adsorbates under chromatographic operating conditions. The new technology will permit the study of mixed-mode retention spectroscopically. 


\section{Duke University Durham, NC 27708}

\section{Department of Chemistry}

\section{Fluorescence Lifetime in Chemical Analysis: The Next Generation McGown, L.B. 919-660-1545}

The two directions in this research are linked by the common thread of new multiharmonic fourier transform (MHF) technology for frequency domain fluorescence lifetime measurements. In the new technique of Total Lifetime Distribution Analysis (TLDA), the commercial MHF instrument will enable rapid fingerprinting and characterization of complex samples with unprecedented sensitivity and detail. The sensitivity derives from the use of the entire fluorescence emission from the sample in the lifetime measurement, and the detail derives from the application of self-modeling data analysis using the Maximum Entropy Method to fully exploit the responsiveness of fluorescence lifetime to the chemical composition and dynamic molecular interactions of a sample. The introduction of the MHF as a rapid, on-the-fly detector of fluorescence lifetime in capillary electrophoresis will increase the accuracy and sensitivity of laser excited fluorescence detection, providing universal applicability through the use of direct and indirect detection schemes, without compromising the speed or resolution that make CE such a valuable technique.

\section{University of Florida Gainesville, FL 32611}

\section{Department of Chemistry}

\section{The Glow Discharge as an Atomization and Ionization Source \\ Harrison, W. 904-392-0780

This research project focuses on fundamental and applied studies of the glow discharge as an analytical source for trace elemental analysis of solid samples by atomic emission, atomic absorption, atomic fluorescence, and mass spectrometry. Of special interest is the use of a radiofrequency discharge for nonconducting samples, thus eliminating the need to mix metal matrices with nonconducting materials to permit dc discharge operation. There is also interest in the chemical plasma reactions that occur under the influence of an rf discharge compared to the dc mode. Complementary techniques of atomic absorption and atomic emission are used to obtain information about sputtering and excitation mechanisms. We are also continuing the work with cryogenic cooling of the glow discharge source to study the effect of trace impurities, such as water vapor, on excitation and ionization processes. A major new direction for this project is the study of a short pulse discharge (microseconds) to power the glow discharge. By employing high power conditions in a short duty-cycle mode, we have seen preliminary results that show attractive emission possibilities. We are also beginning to assemble a time-of-flight mass spectrometer to take advantage of the short pulse characteristics of the new source. In addition, short term injection of atoms and ions in this mode permits us to study discharge reactions and diffusion processes that are impossible to explore with continuous or longer pulse methods.

\section{Development of Laser-Excited Atomic Fluorescence and Ionization Spectrometric Methods \\ Winefordner, J.D. 904-392-0556}

$\$ 109,500$

The emphasis in this research is upon the development of new, sensitive, selective spectroscopic methods for trace elemental analysis. Several projects are ongoing that involve ionization, emission, and fluorescence in flames and glow discharges. Laser enhanced ionization (LEI) in a microflame is being studied with two means of sample introduction: ultrasonic nebulization, which will permit a thorough optimization of burner design and flame gas composition, and laser ablation, which will provide the capability of analyzing single small particles and for elemental mapping of surface and depth profiles. LEI is also being studied as a high resolution spatial spectroscopic probe for flame temperatures (via detection of $\mathrm{OH}$ fluorescence) and for the in situ detection of $\mathrm{N}_{2}$ via measurement of Raman scattering. Glow discharge atomic reservoirs are also being studied using both emission spectrometry with a microcavity hollow cathode discharge and laser excited atomic fluorescence for a micro-planar discharge. Both of these glow discharge systems accept small volume discrete samples. The emission system has multielement capability on subsample volumes, and the fluorescence system has the potential to approach single atom detection in a real sample.

\section{The George Washington University Washington, DC 20052}

\section{Department of Chemistry}

\section{New High-Temperature Plasmas and} Sample Introduction Systems for Analytical Atomic Emission and Mass Spectrometry

Montaser, A. 202-994-6480

This research follows a multifaceted approach, from theory to practice, to the investigation and development of novel helium plasmas, sample introduction systems, and diagnostic techniques for atomic and mass spectrometries. Four major sets of research programs are being conducted that each include a number of discrete but complementary projects. The first program is concerned with investigation of atmospheric-pressure helium inductively coupled plasmas (He ICPs) that are suitable for atomization and ionization of elements, especially those possessing high ionization energies, for the purpose of enhancing the detecting powers for a number of elements. The second program includes simulation and computer modeling of He ICPs. The aim is to ease the hunt for new helium plasmas by predicting their structure and fundamental and analytical properties, without incurring the enormous cost for extensive experimental studies. The third program involves spectroscopic imaging and diagnostic studies of plasma discharges to instantly visualize their prevailing 
structures, to quantify key fundamental properties, and to verify predictions by mathematical models. The fourth program entails development and characterization of new, low-cost sample introduction systems. These efforts collectively offer promise of solving singularly difficult analytical problems that either exist now or are likely to arise in the future in the various fields of energy generation, environmental pollution, biomedicine, and nutrition.

\section{Hampton University Hampton, VA 23668}

\section{Department of Chemistry}

\section{Use of Ion Chromatography-dc Plasma Atomic Emission Spectroseopy for the Speciation of Trace Metals Urasa, I,T. 804-727-5398 \\ $\$ \mathbf{5 7}, \mathbf{5 0 0}$}

The research has focused on studies of the solution chemistry and speciation of trace metals using chromatographic coupled with spectroscopic techniques, The new thrust is directed towards incorporating solid phase extraction in metal speciation. Application of solid phase extraction in analytical measurements has several advantages, including; isolation of the analyte from complex sample matrix; sample preconcentration which, for dilute samples, leads to improvement in measurement sensitivity; and the possibility of some separation of the chemical species present. Several sorbents, including bonded reversed phase, bonded normal phase, and naturally occurring polymers will be studied and characterized for the extraction of neutral, charged, and hydrated metal species in solution. The extraction mechanisms involved in each case will be elucidated, Attempts will also be made to modify sorbent functionality by derivatization, thereby placing on the adsorption site desired functionalities to achieve selectivity in the extraction and speciation process. While limited separation can occur on the solid phase extraction tube, analytical separation of the extracted metal species will be achieved by the elution of the extracted species on a chromatographic column connected to the extraction tube.

\section{University of Illinois at Urbana-Champaign Urbana, IL 61801}

\section{School of Chemical Sciences}

\section{Molecular Aspects of Transport in Thin Films of Controlled Architecture Bohn, P.W. 217-333-0676 \\ $\$ 86,000$}

Augmentation of localized vibrational spectroscopy experiments with measurements of plasmon surface polarity (PSPs) in Ag- or Au-supported ultrathin ( $d \leq 35 \mathrm{~nm}$ ) poly(methyl methacrylate) films exposed to $\mathrm{CH}_{3} \mathrm{O} H$ has occurred in the past year. Measurements of shifts in PSP resonances are exquisitely sensitive, ultimately permitting changes in film thickness as small as Delta $d \leq 0.01$ $\mathrm{nm}$ to be detected. These measurements allowed to build a detailed picture of the changes in the dielectric function of the polymer as it changes from the glassy to rubbery state, It is important that these films are sufficiently thin so that the entire film is interfacial, allowing the direct study of how the polymer responds to the osmotic stress that is established at the glassy-rubbery boundary and how the induction time in Case II diffusion is related to molecular properties and details of film processing. Initial measurements in the $\mathrm{PMMA} / \mathrm{CH}_{3} \mathrm{OH}$ system confirm the form of the adsorption isotherm in a modified Thomas-Windle model, while allowing numerical values to be assigned to many parameters that heretofore have been inaccessible

\section{Kansas State University Manhattan, KS 66506}

\section{Department of Chemistry}

\section{Multidimensional Hadamard Transform Spectrometry: A New Analytical Technique Hammaker, R.M.; Fateley, W.G. $\quad \mathbf{\$ 8 5 , 0 0 0}$ 913-532-1454}

Multidimensional spectrometry is defined in terms of three spatial dimensions $\left(x_{i}, y_{i}, z_{i}\right)$ and one spectral di= mension (wavelength, $\lambda_{j}$ or frequency, $v_{j}$ ). The $x_{i}$ and $y_{i}$ or surface coordinates are accessed via a stationary twodimensional (2-D) Hadamard encoding mask and the $\mathrm{z}_{\mathrm{i}}$ or depth coordinate arises from using a photoacoustic detection system for depth profiling by optical modulation and phase-sensitive detection. Measurements utilizing twodimensional and three-dimensional spectrometry are in print. Results for this program's first images and spectra from four-dimensional spectrometry are in press. Present efforts are focused on enhancement of capabilities for performing yarious multidimensional spectrometries. A multiwavelength acoustic-optic tunable filter (AOTF) for the spectral dimension is in its final testing. $A$ new moving 2-D Hadamard encoding mask as a potential replacement for the stationary 2-D Hadamard encoding mask for the two surface dimensions has been obtained and is now being evaluated. Decoding the multiwavelength AOTF results by Hadamard methods gives a multiplex adyantage in the spectral dimension. Using the multiwavelength AOTF without decoding will proyide a multiplication advantage in images without spectral separation. The moving mask with completely open windows will allow access to any spectral region where appropriate sources, spectral separators, and detectors are available.

\section{Lehigh University Bethlehem, PA 18015}

\section{Department of Chemistry}

\section{Perforated Monolayers Regen, S.L. $610-758-4842$}

$\$ 74,000$

This program is aimed at preparing new classes of synthetic membranes that can be used to separate small molecules on the basis of their size, shape, and polarity. The general approach that is being taken is to fabricate composite membranes from "perforated monolayers" (i.e., 
monolayers that are assembled from "porous surfactants") plus highly permeable substrates such as cast films of poly[1-(trimethylsilyl)-1-propyne] (PTMSP). Research that has been carried out to date has led to the synthesis and characterization of a homologous series of calix[n]arenebased surfactants that differ in their internal diameter. Current efforts are now focusing on (i) the fabrication of composite membranes from such amphiphiles by use of Langmuir-Blodgett (LB) methods; (ii) the characterization of their barrier properties toward $\mathrm{He}, \mathrm{N}_{2}$, and $\mathrm{SF}_{6}$; and (iii) the clarification of the importance of transfer speed and surface pressure (during LB deposition) on the permeation selectivity (permselectivity), and reproducibility of these composite membranes.

\section{Louisiana State University Baton Rouge, LA 70803}

\section{Department of Chemistry}

\section{Novel Micellar and Calixarene Derivatives for Selective Luminescence Measurements \\ Warner, I.M. \\ 504-388-2829 \\ $\$ 96,000$}

This research focuses on selective measurement of polar aromatic compounds. The proposed research is divided into two major areas. The first is the continuation of our studies in the area of novel synthetic reversed micellar systems for selective and improved sensitized luminescence detection of polynuclear aromatic hydrocarbons containing polar substituents. Experiments and data from our previous grant demonstrate that detection of these compounds is not only viable, but also highly selective. The second area of our proposed research involves continued studies of calixarenes as host molecules for analytical measurements. We desire to explore these molecules for their guest:host capabilities and for selective sensitized luminescence measurements. Our studies will also focus on the ternary complexes of the calixarenes. The goal of this component of our research is to examine the similarity of the calixarenes to the cyclodextrins in terms of their binding abilities. In addition to solution studies, we propose to use the calixarenes for selective separations in the area of capillary electrophoresis.

\section{Michigan State University East Lansing, MI 48824}

\section{Department of Chemistry}

\section{Direct Examination of Separation Processes in Chromatography by Laser-Induced Fluorescence McGuffin, V.L. 517-355-9715}

The objective of this research program is to improve the understanding of fundamental hydrodynamic and physicochemical phenomena in separation science. During this period, a three-dimensional stochastic computer simulation has been developed and validated which provides a unified treatment of gas, supercritical fluid, and liquid chromatography. In this simulation, the migration of individual molecules is established through diffusion and convection within a fluid phase that is in contact with a surface. Molecular interaction and, hence, retention may arise by partitioning into permeable surfaces or by adsorption at solid surfaces. The molecular distribution and the corresponding zone profile may be examined and characterized by means of statistical moments at any specified time or spatial position during the simulation. Because this simulation monitors the migration of individual molecules, it provides the opportunity to perform hypothetical experiments and to make observations that may not be possible in a real chromatographic system. For example, the kinetic rate constants may be directly determined and their dependence upon variables such as the column radius, film thickness, distribution coefficient, and diffusion coefficients may be readily established. In addition, retention and dispersion processes may be examined in regions of discrete spatial transition (e.g., injection and elution) as well as discrete or continuous temporal transitions (e.g., temperature, mobile-phase composition, or velocity gradient). Thus, this simulation provides a powerful and versatile model with which to characterize transport phenomena in complex chromatographic separation systems.

\section{University of Missouri at Rolla Rolla, MO 65401}

\section{Department of Chemistry}

\section{A New Class of Macrocyclic Chiral Selectors for Stereochemical Analysis Armstrong, D.W. 314-341-4429

There are a large number of macrocyclic antibiotics that encompass a variety of different structures. Many of these compounds have the type of functional groups and the spacial arrangement needed for enantioselective interactions. We have worked with over a score of these macrocycles. The efficacy of four of them (in terms of enantiomeric separations) will be discussed. Their usefulness as chiral selectors in LC and particularly in CE appears to exceed that of many other common selectors. For example, CE resolution factors (Rs) for many enantiomers are in the 5 to 20 range. The chiral selectors to be discussed are: rifamycin B (an ansa compound), vancomycin, ristocetin A, teicoplanin, and thiostreptin (a cyclic peptide). Each of these compounds are ionizable but have different isoelectric points and differ in size and selectivity. Although these macrocycles are among the newest chiral selectors, they have been successfully utilized in hundreds of enantiomeric separations. Some mechanistic aspects of separations involving macrocyclic antibiotics will be discussed. As expected, when these macrocycles are used as mobile phase additives in chromatography or as chiral selectors dissolved in solution for $\mathrm{CE}$, they are treated using the pseudophase model that we developed for micelles and cyclodextrins over 14 years ago. Several research groups have "derived" and re-expressed (a euphemism for rearranging a known equation and using different symbols so that it looks original) equations that use electrophoretic parameters to calculate association constants to chiral selectors and/or micelles. As will be shown, these basic 
equations have been reported previously many times dating back at least to 1951. Despite this, some prominent groups in the CE field continue to report incorrect numbers using incorrect equations and/or assumptions.

\section{National Institute of Standards and Technology, Gaithersburg Gaithersburg, MD 20899}

\section{Chemical Kinetics and Thermodynamics Division}

\section{Ion Kinetics and Thermochemistry Pertinent to Direct Mass Spectrometric Organic Speciation \\ Sieck, L.W. 301-975-2563

This project involves the measurement of fundamental properties of gas phase ions. The National Institute of Standards and Technology (NIST) pulsed electron beam mass spectrometer (MS) is the prime experimental facility, and a Fourier transform ion cyclotron resonance. (FTICR) instrument and triple MS system are also available. Emphasis is in two areas. One area is definition of the thermochemistry of associated and cluster ions via variable-temperature equilibrium measurements. Correlation lines, which express the dependence of bond strengths upon the differences between the acidities or proton affinities of the two interacting partners, are then developed for predictive purposes for use in experimentally inaccessible systems. These investigations are augmented by collaborative efforts involving the study of intracluster ion-molecule chemistry occurring after ionization of neutral organic clusters produced in expansion beams. The second area involves extension and clarification of earlier kinetic studies from other laboratories to provide a more accurate database for gas phase ion and neutral thermochemistry. Currently, research includes extensive measurement of proton transfer equilibria as a function of temperature to provide interlocking thermochemical ladders which, when referenced to primary standards, provide absolute proton affinity and acidity scales. Related studies include ion pyrolysis and ion kinetics pertinent to plasma etching and electric breakdown in gaseous dielectrics.

\section{University of North Carolina at Chapel Hill Chapel Hill, NC 27599}

\section{Department of Chemistry}

\section{Solid-State Voltammetry and Sensors in Gases and Other Nonionic Media Murray, R.W. 919-962-6296

This project is based on using microelectrode voltammetry for design and application of quantitative electrochemical transport and kinetics experiments to semi-solid and solid redox phases. The experiments include transport and electron transfer reactions of electron donor/acceptor solutes and surfaces in polymeric solvents and liquid crystalline phases. The goals of this project include (i) developing the necessary miniaturized electrode methodologies, cells, and requisite theory for quantitative voltammetry in rigid media, (ii) exploring important characteristics of homogeneous and heterogeneous electron transfer reaction dynamics in rigid environments, in particular how the dynamics of "outer sphere" redox couples respond to rigidification of their surroundings, (iii) exploring polymerphase transport, including polymer-in-polymer diffusion of redox-labelled poly-ethers, anisotropic transport in liquid crystalline phases and polymers, and coupling between slow diffusion and homogeneous electron transfers, and (iv) learning to dynamically manipulate diffusion rates of redox sites within polymer electrolytes so as to fashion ultrathin, electrically conducting mixed valent layers by freezing the concentration gradients that are electrolytically generated at electrodes. We have recently succeeded in fashioning an example of a frozen gradient system, from a poly-ether-derivatized viologen.

\section{Purdue University West Lafayette, IN 47907}

\section{Department of Chemistry}

\section{Ion Trap Mass Spectrometry: Ion Motion, Reactions, and Applications \\ Cooks, R.G. \\ 317-494-5262 \\ $\$ 150,000$ \\ (18 months)}

Fundamental understanding of ion motion in the quadrupole ion trap is being sought by (i) laser tomography experiments which yield temporal and spatial resolution, (ii) dc pump/laser probe experiments which accelerate ions and characterize their frequencies, and (iii) novel methods of manipulating ions, including phaselocked resonant excitation, dc pulse activation, and stored waveform methods of ion ejection. Simulations of the motion of ensembles of ions, which include collision effects and ion-ion interactions, are being refined. Experiment and simulation are being applied: (i) to evaluate capabilities for measuring ion polarizability, (ii) to characterize mass shifts due to coulombic ion-ion interactions, (iii) to attempt to disperse ions by differences in ion mobility arising from different collision cross sections, and (iv) to characterize new methods of mass analysis including the use of quadrupolar dc ejection during ac activation for improved mass resolution. Fourier transforms of the induced image currents are being used to perform non-destructive ion detection. Particular chemical systems of interest include (i) the fullerenes, where electron transfer is of interest, and (ii) phenoxyl and other free radicals, the thermochemical properties of which are being measured by cluster ion dissociation.

\section{Reactions of Gaseous Metal Ions/Their Clusters in the Gas Phase Using Laser Ionization: Fourier Transform Mass Spectrometry \\ Freiser, B.S. 317-494-5270 \\ $\$ 85,000$}

The research group continues to use Fourier transform ion cyclotron resonance (FTICR) mass spectrometry to study the chemistry and photochemistry of metal-containing ions in the gas phase. Some areas of current interest are 
summarized here: (1) the reactions of $\mathrm{MC}_{60+}$, where $\mathrm{M}$ is an externally bound transition metal, with a variety of reagents are being investigated with a particular focus on using the metal center to initiate the derivation of $\mathrm{C}_{60}$. (2) A new class of distonic ions (ions in which the charge and the radical sites are spacially separated, of intense current interest) has been demonstrated with the discovery of the organometallic ion radical ${ }^{+} \mathrm{FeC}_{6} \mathrm{H}_{4}$. The properties of this species and its analogs are being probed, and other examples being sought. (3) Studies of small metal cluster ions are continuing with a focus on metal-carbon clusters, popularized by the discovery of metallocarbohedrenes $\left(\mathrm{M}_{8} \mathrm{C}_{12}: \mathrm{M}=\right.$ transition metal) by Castleman and coworkers. Structure - reactivity relationships are being developed in collaboration with several theorists. (4) A new direction we will be pursuing is the measurement of slow metastable dissociations in organometallic ions using a recently reported FTICR kinetic approach. This technique holds promise for understanding mechanisms by which multiple reductive eliminations occur.

\section{Rensselaer Polytechnic Institute Troy, NY 12180}

\author{
Department of Chemical Engineering
}

\section{Chemical Interactions betẅeen Protein Molecules and Polymer Membrane Materials \\ Belfort, $G$. 518-276-6948

To help understand the effects of protein adsorption on membrane filtration performance, the molecular interactions between cellulose acetate films and two proteins with different properties (ribonuclease $A$ and human serum albumin) have been measured with the Surface Force Apparatus. Comparison of forces between two protein layers with those between a protein layer and a cellulose acetate (CA) film, shows that at high $\mathrm{pH}$, both proteins retained their native conformation on interacting with the CA film while at the isoelectric point $(\mathrm{pI})$ or below, the tertiary structure of proteins was disturbed. Similar results have recently been obtained with hen egg lysozyme and polysulfone films. These measurements provide the first molecular evidence that disruption of protein tertiary structure could be responsible for the reduced permeation flows observed during membrane filtration of protein solutions and suggest that operating at high $\mathrm{pH}$ values away from the pI of proteins will reduce such fouling. To reduce such attractive interactions, the surface of commercial polyacrylonitrile ultrafiltration membranes has been modified using low-temperature plasma.

\section{State University of New York at Buffalo Buffalo, NY 14260}

\section{Department of Chemistry}

\section{Determination of Solvation Kinetics in Supercritical Fluids \\ Bright, F.V. \\ $716-645-6800$ \\ $\$ 65,000$}

This project is directed toward determining the effects of supercritical fluids on solvation and chemical reactions. The main goals are (1) understanding the effects of neat and entrainer-modified supercritical fluids on solute-fluid interactions; (2) determining the influence of fluid density on the disposition of energy between a dissolved solute and the fluid bath; and (3) probing the effects of continuous phase density on the internal dynamics of reverse micelles formed in supercritical fluids. In order to follow these fast molecular-level processes, modern picosecond in situ optical spectroscopy is being used. To date this work has (1) helped define how "diffusion controlled" reactions proceed in supercritical fluids; (2) determined how and on what time scale entrainers preferentially solvate solutes in the near-critical region; (3) shown that the conformation (i.e., reactivity) of flexible solutes can be altered easily with fluid density; (4) studied how lateral diffusion within reverse micelles (formed in supercritical alkanes) is affected by the continuous phase density; (5) probed how the conformation of polymers can be controlled with supercritical fluids; and (6) determined how fluid density can be used to affect the radiative and nonradiative rates of dissolved solutes in supercritical fluids. This new information is leading to a better molecular-level understanding of the unique chemistry of supercritical fluids and how fluids can be used to control reactions and solute conformation'(i.e., reactivity).

\section{Syracuse University Syracusè, NY 13244}

\section{Department of Chemical Engineering and Materials Scienice}

\section{Mechanisms of Gas Permeation through Polymer Membranes \\ Stern, S.A. \\ 315-443-4469

The objective of this study is to investigate the mechanisms of gas transport in polymers by molecular dynamics simulations. It is desired, in particular, to determine the effects of systematic changes in polymer structure on the gas transport. Accordingly, diffusion coefficients of $\mathrm{He}, \mathrm{O}_{2}$, $\mathrm{N}_{2}, \mathrm{CO}_{2}$, and $\mathrm{CH}_{4}$ in poly(dimethyl siloxane) (PDMS), poly(propyl methyl siloxane), poly(trifluoropropyl methyl siloxane), and poly(phenyl methyl siloxane) were calculated for a temperature of $300 \mathrm{~K}$. The diffusion coefficients decrease with decreasing intrasegmental mobility of the polymer chains (i.e., with increasing bulkiness of side chains and increasing glass-transition temperature) as well as with increasing "kinetic" diameter of the penetrant molecules. The calculated values of the diffusion coefficients for all gas/polymer systems studied are consistent 
with experimental values within the expected error limits. An analysis of the trajectories of the penetrant molecules in the polymers revealed two types of motions: (1) "oscillating motions" inside cavities in the polymer matrix, and (2) "jumps" from one polymer cavity to another one. The lengths of the jumps are of the order of 8-10 $\AA$, whereas the oscillating motions are of the order of $<5 \AA$. The diffusion of gases in polymers is controlled by the fractional free-volume in the polymer, the free-volume distribution, and the dynamics of the free-volume. Preliminary simulations indicate that the most highly gas-permeable polymer, namely PDMS, has a larger mean free volume and a broader distribution of free-volume voids than the other poly(organosiloxanes) studied. As a result, PDMS also has the lowest selectivity for different diffusing gases.

\section{University of Tennessee at Knoxville Knoxville, TN 37996}

\section{Department of Chemistry}

\section{Polymer-Based Separations Synthesis and Application of Polymers for Ionic and Molecular Recognition \\ Alexandratos, S.D. 615-974-3399

The synthesis of polymer-supported reagents for the selective complexation of metal ions and neutral molecules in multicomponent solutions is an area of continuing importance due to environmental concerns at many operations, including Department of Energy facilities. This research has centered on the synthesis of polymer-supported reagents with more than one type of ligand immobilized on the network. The goal is to understand the role of multisite cooperation in enhancing specificity in polymersubstrate interactions. During the current period, we have synthesized and studied (a) bifunctional polymers with immobilized ligands, one of which (studied in collaboration with Argonne National Laboratory) is now commercially available as Diphonix ${ }^{\mathrm{TM}}$ ion exchange resin and is a 1994 recipient of a R \& D-100 Award; and (b) bifunctional polymers where one type of ligand is immobilized on the network and a second type remains mobile'(in collaboration with the Oak Ridge National Laboratory). Additionally, solid-state NMR (both ${ }^{13} \mathrm{C}$ and ${ }^{31} \mathrm{P}$ ) has been introduced as a means of characterizing bifunctional polymers, Most recently, the study has begun exploring the synthesis of polymer-supported reagents as catalysts in organic chemical reactions, focusing on the aldol and Mitsunobu reactions.

\section{Study of the Surface Properties of Ceramic Materials by Chromatography Guiochon, G.} 615-974-0733

The aim of this project is the characterization of surfaces by the adsorption energy distributions of a number of selected probe compounds. The procedure followed involves the determination of the adsorption isotherms of the probes from which the adsorption energy distributions are calculated. Adsorption isotherms are measured by a chromatographic procedure, using open tubular columns whose walls are coated by a layer of the powder. This method permits an accurate determination of the isotherms. The adsorption energy distribution is derived from the isotherm by numerical solution of an integral equation relating it to the local adsorption isotherms and to the measured isotherm. Classical procedures are inaccurate because they introduce bias and artifacts. A new procedure has been developed that avoids these errors. Simulations of the measurement process have shown that the isotherms must be determined in an unusually wide range of partial pressures. This causes experimental problems that are being solved. Measurements are carried out using silica powders and various silica surfaces modified by chemical reactions. However, the adsorption energy distribution of chemically bonded silicas does not extend far into the high energy range. This makes experimental determinations difficult and inaccurate with this kind of material.

\section{Capillary Electrokinetic Separations with Optical Detection \\ Sepaniak, M.J. \\ 615-974-8023 \\ $\$ 81,000$}

This multifarious research program is dedicated to the development of capillary electrokinetic separation techniques and associated optical methods of detection. Currently, research is directed at three general objectives. First, fundamental studies of pertinent separation and band broadening mechanisms are being conducted, with the emphasis on understanding systems that include highly ordered assemblies as mobile phase additives. The additives include cyclodextrins (CDs), affinity reagents (e.g., immunochemicals), soluble polymers (as size-sieving reagents), and fullerenes. These are employed in electrophoretic (e.g., capillary electrophoresis and/ or electrochromatographic (e.g., micellar electrokinetic capillary chromatography [MECC] and capillary electrochromatography [CEC]) modes of separation. Reasonable correlations between molecular modeling-based cyclodextrin-solute interaction energies and retention behavior have been observed for certain mixtures of geometric isomers and optical isomers. In addition, nonaqueous CEC separations of common fullerenes have been performed for the first time. Recently, a dual-CD (charged and neutral) form of capillary electrokinetic chromatography has been demonstrated that exhibits unique and beneficial characteristics relative to MECC. Second, the analytical methodologies associated with these capillary separation methods are being advanced through the development of retention programming (with simplex optimization) techniques and new strategies for performing optical detection. With regard to the latter, the advantages of laser fluorimetry are extended through the inclusion of fluorogenic reagents (e.g., DNA interculation dyes) in the mobile phase. Recently, a novel instrumental configuration for capillary electrokinetic separations has been developed that permits rapid movement of the detection zone. Chemiluminescence detection using ultralow volume, molded postcapillary reactors has been demonstrated. Third, successful development of these separation and detection systems will fill current voids in the capabilities of capillary separation techniques. In particular, it should be possible to perform highly efficient and selective separations of hydrophobic compounds (e.g., higher MW polycyclic aromatic hydrocarbons), mixtures of chiral compounds, DNA fragments, and fullerene mixtures. In a new area of analytical research, fiber optic sensors are being 
developed that include electrokinetic separation mechanisms (e.g., capillary electrophoresis at the fiber terminus) to enhance measurement selectivity. A frontal electrophoretic separation of laser dyes, using a first generation sensor, has been performed to demonstrate feasibility.

\section{Texas A \& M University College Station, TX 77843}

\section{Department of Chemistry}

\section{Development of Laser-Ion Beam Photodissociation Methods Russell, D.H. 409-845-3345}

This research program focuses on numerous aspects of laser-mass spectrometry and fundamental gas-phase ion chemistry. Photodissociation methods are being developed to probe dynamics of dissociation reactions of highly activated ionic systems. In addition, the potential analytical utility of laser-ion photodissociation for structural characterization of large biomolecules is being evaluated. A photodissociation apparatus is now being used for a range of tandem mass spectrometry experiments. The ions are formed by pulsed UV laser desorption (matrix-assisted laser desorption ionization [MALDI]), and photodissociation of the mass-selected ion is performed by using a high-power, pulsed eximer, Nd:YAG or $\mathrm{N}_{2}$ laser. The primary objective of the current research is to improve the sensitivity of MS-MS experiments by 100 times (10 to 100 femtomole) and the mass resolution of MS-II by 5 to 10 times $(1,000$ to 10,000$)$. The objective of the TOF/TOF experiment is to study the dissociation reactions of very large ( $>\mathrm{m} / \mathrm{z}$ 5000) molecules. In addition, laser-ion beam photodissociation methods are being used to examine ionic clusters that are important to matrix-assisted-UVlaser desorption ionization of polar, thermally labile biomolecules. In particular, studies are conducted on excited state $\mathrm{H}^{+}$-transfer reactions and the way in which such reactions influence the dissociation chemistry of gasphase ionic systems.

\section{Texas Tech University Lubbock, TX 79409}

\section{Department of Chemistry and Biochemistry}

\section{Metal Ion Complexation by Proton-Ionizable Lariat Ethers and Their Polymers \\ Bartsch, R.A. 806-742-3069

Objectives of this research are the preparation of new metal ion complexing agents and chelating polymers and their applications in metal ion separation processes. Cyclic polyethers (crown ethers), which possess pendent, proton-ionizable functional groups, are novel agents for metal ion separations by solvent extraction and liquid membrane transport processes. Movement of the metal ion from an aqueous phase into the organic phase does not require concomitant transport of an aqueous phase anion.
This factor greatly increases metal ion extraction and transport efficiency compared with that of structurally related non-ionizable crown ethers in separations involving metal chlorides, nitrates, and sulfates. New crown ether carboxylic acids, phosphonic acid monoethyl esters, phosphonic acids, and sulfonic acids are being synthesized and tested to probe the influence of structural variation within the ligand upon the selectivity and efficiency in separations of alkali and alkaline earth metal ions. Novel chelating polymers are being prepared by condensation polymerization of proton-ionizable dibenzocrown ether monomers. In addition to ion-exchange sites, these resins also provide crown ether units for metal ion complexation. Sorption behavior of these resins for a variety of alkali metal, alkaline earth, and heavy metal cations is being assessed.

\section{Seperations on Water-Ice Dasgupta, P.K. 806-742-3064}

$\$ 100,000$

This project seeks to explore the merits of ice as a separation medium. Since such a stationary phase is easily and inexpensively produced, even modest separation efficiencies can have important impact on large scale separations. As many as possible of the following will be investigated in the project time frame: (a) the use of ice as a chromatographic medium in columnar form in liquid and gas chromatography, (b) the use of ice as a separation surface for carrying out slab electrophoresis, (c) the use of ice as a migration medium to carry out zone electrophoresis, (d) the use of ice in the separation of ionic vs. nonionic solute classes, and (e) the use of ice as a zone melting separation medium, taking advantage of the pressure-freezing point relationships of water.

\section{University of Texas at Austin Austin, TX 78712}

\section{Department of Chemical Engineering}

\section{Synthesis and Analysis of Novel Polymers with Potential for Providing Both High Permselectivity and Permeability in Gas Separation Applications \\ Koros, W.; Paul, $D$. 512-471-5866 \\ $\$ 89,000$}

Thermally stable gas separation membrane materials having high levels of aromaticity, low degrees of internal rotation but a bulky character with respect to interchain packing will be developed in this project. Future membrane formation processes may require a reactive step to achieve the necessary segmental rigidity and segmental packing distributions needed to generate super selective materials based on entropic as well as energetic mobility selective factors. This aspect will be an important focus of the work being carried out here. In this case, convenient solution-based membrane formation processes currently used can still be relied upon while also achieving a final molecular sieve-like or highly crosslinked selective skin environment. Thermal, chemical, and irradiation post treatments will be studied. Elevated temperature studies above $200^{\circ} \mathrm{C}$ will be performed for new applications such as membrane-assisted reactors. These thermal characterizations will also allow factoring of the energetic and 
entropic contributions to mobility selectivity in these unusual materials.

\section{University of Utah Salt Lake City, UT 84112}

\section{Department of Chemistry}

\section{Laser Flash Photolysis, EPR, and Raman Studies of Liquids at Elevated Pressures Eyring, E.M. 801-581-8658 $\$ 80,000$}

Rates and mechanisms of chemical reactions in liquids and in supercritical fluids are being elucidated by several experimental techniques that permit the variation of pressure between one atmosphere and 1500 atmospheres. In one experiment a frequency tripled Nd:YAG laser delivers a pump pulse to a sample cell containing either molybdenum hexacarbonyl or tungsten hexacarbonyl and one of several bidentate ligands dissolved in a solvent. Solvents include liquid toluene, liquid carbon dioxide, supercritical carbon dioxide, and supercritical ethane. The rate constant for a thermal ring closure reaction is determined at several pressures leading to a volume of activation for the reaction. From the very large positive volume of activation found just above the critical point the participation of many solvent molecules in the formation of the activated complex is deduced. A high-pressure stopped-flow spectrophotometer is being used to determine rates and mechanisms of complexation reactions between lanthanide ions and DTPA or DTPA derivatives in aqueous solutions. The ability of lignin peroxidase dissolved in a supercritical solvent to bioremediate contaminated soils is also being explored. The use of a high pressure electron spin resonance sample cell is being investigated as well as the use of a high-pressure sample cell for cyclic voltammetry.

\section{Time-Resolved Analytical Methods for Liquid/Solid Interfaces \\ Harris, J.M. \\ 801-581-3585}

Many chemical phenomena that occur at the boundaries between insulating solids and liquids (adsorption, partition, monolayer self-assembly, catalysis, and chemical reactions) are critical to energy-related analytical chemistry, These phenomena are central to the developing of chromatographic methods, solid-phase extraction, immobilized analytical reagents, and optical sensors. The goal of this program is, therefore, to develop surface-sensitive spectroscopies by which chemical kinetics at liquid/ solid interfaces can be observed on timescales from nanoseconds to seconds. Temperature-jump relaxation measurements are used to probe adsorption/desorption kinetics at liquid/solid interfaces; kinetic barriers to adsorption of molecular ions onto alkylated silica surfaces are being investigated. The migration of covalentlyattached ligands on silica surfaces has been observed, and from the temperature-dependence of the diffusion rate, the large energy barrier to migration is estimated. Surface heterogeneity of adsorption sites on silica was recently examined by time-resolved fluorescence, and its chemical origins investigated by $\mathrm{Si}^{29} \mathrm{nmr}$ spectroscopy. Surfaceenhanced Raman spectroscopy is used to study adsorption and binding to silica surfaces. Molecular dynamics simulations are being carried out to help better understand kinetic barriers to adsorption; esr probe measurements were launched to measure the mobility of silica-attached alkyl ligands on a subnanosecond timescale.

\section{Department of Metallurgical Engineering}

\section{Hydrophobic Character of Nonsulfide Mineral Surfaces as Influenced by Double Bond Reactions of Adsorbed Unsaturated Collector Species \\ Miller, J.D. 801-581-5160 \\ $\$ 100,000$}

The research efforts are directed toward establishing the fundamental features of surfactant adsorption phenomena in nonsulfide mineral flotation systems in order to develop more energy-efficient separations. Froth flotation is one of the most important examples of applied surface chemistry and this separation technology is used in the food, petroleum, pulp-paper, and mineral industries. Several novel experimental techniques such as in situ Fourier transform infrared (FTIR) internal reflection spectroscopy (IRS) with reactive internal reflection elements, multichannel laser Raman spectroscopy, nonequilibrium laser-Doppler electrophoresis, and atomic force microscopy for surface force measurements are being developed and/or used to analyze surfactant adsorption phenomena at mineral/water interfaces and to describe the impact of adsorption phenomena on the hydrophobic character of the surface. The nonsulfide flotation research includes the soluble salt, semisoluble salt, and insoluble oxide mineral systems. The surface charge/surfactant colloid adsorption model initially developed to explain the flotation behavior of alkali halides has now been used to understand and improve the flotation behavior of other soluble salt minerals including the recovery of double salts from their saturated brines and borax minerals. Atomic force microscopy in concert with FTIR/IRS techniques is currently being used to investigate the influence of the structure of interfacial water on the short-range interparticle forces responsible for the adsorption of surfactant colloids in soluble salt flotation. Significant progress continues to be made in spectroscopic characterization of interfacial water at both hydrophilic and hydrophobic surfaces by depth profiling using in situ FTIR/RS. In the category of semisoluble salt minerals, carboxylic acid adsorption by fluorite, calcite, and apatite minerals is being examined in situ by FTIR/IRS and Raman spectroscopy in order to determine the effect of bulk solution calcium concentration on surfactant adsorption at these mineral surfaces. Studies on adsorption kinetics using in situ FTIR/IRS are in progress to determine the activation energies of oleate and linoleate surfactant adsorption at the fluorite surface. In addition, linear dichroism spectroscopy is being used to examine both self-assembled and transferred LB films to determine the influence of molecular orientation on the structure of the adsorbed surfactants at mineral surfaces and the hydrophobic state. Several insoluble oxide mineral systems including sappire/sodium dodecyl sulfate and quartz/amine are being examined to describe collector adsorption phenomena with respect to adsorption density, adsorption state, and the order/ conformation of adsorbed surfactant species. The effect of surface hydration and interfacial water structure as determined from surface spectroscopy will provide further 
information on the relationship between collector adsorption phenomena and hydrophobic state. The results obtained from this research program will provide the basis for new reagent schedules to improve flotation separation efficiency and to promote energy conservation.

\section{Virginia Commonwealth University Richmond, VA 23284}

\section{Department of Chemistry}

397. Selective Methods for Quantification of
Target Species in Complex Mixtures
Rutan, S.C.
804-828-7517

The quantification of specific compounds in complex mixtures is a common goal in many analytical methods. Here, two experimental approaches are coupled with chemometric data analysis methods to explore the possibility of obtaining reliable, quantitative results subsequent to chromatographic separations. The first approach is liquid chromatography coupled with UV-visible diode array and mass spectral detection for the analysis of polyaromatic hydrocarbons, metabolites, and pesticide residues. Quantification will be achieved by using methods such as rank annihilation, adaptive filter, direct trilinear decomposition, and neural networks. Standard addition methods can be used for calibration. In the second experimental approach, thin-layer chromatography will be used to separate the analytes, and the kinetics of a subsequent derivatization reaction will be used to resolve and quantify the species of interest on the thin-layer plates. This novel approach has been applied successfully for the analysis of amino acids, and the trilinear decomposition method has given satisfactory results for the quantitative resolution of the severely overlapped amino acids, glutamine and glycine. Chemiluminescent-detection approaches based on these principles will also be investigated.

\section{Washington State University Pullman, WA 99164}

\section{Department of Physics}

\section{UV Laser-Surface Interactions Relevant to Analytic Spectroscopy of Wide Bandgap Materials \\ Dickinson, J.T. 509-335-4914

This research emphasizes basic studies of the laser desorption and ablation of materials, in particular, those with bandgaps that exceed the photon energies of the incident light. The mechanisms of emissions and formation of ground state and excited neutral species, ions, and free electrons are probed using time-resolved optical spectroscopy, photoluminescence, charged particle energy analysis, and angular distribution measurements. The existence and production of point defects and their role in (a) photodesorption processes, (b) heating and vaporization, and (c) plasma formation is of current interest. In several materials, laser- induced dislocation motion is a primary source of vacancy defects in the near surface region of wide bandgap materials. Current studies involve imaging and quantifying defect densities, the production of surface defects with particle bombardment, modeling the role of anion vacancies in photostimulated emission of cations, and studies of laser interactions with inorganic solids containing covalently bonded anions that photodecompose.

\section{University of Wyoming Laramie, WY 82071}

\section{Department of Chemistry}

\section{Solid-Matrix Luminescence Analysis Hurtubise, R.J. 307-766-6241}

The major priority in this project is the development of a basic understanding of the interactions that are needed for the room-temperature fluorescence and phosphorescence of aromatic compounds adsorbed on solid matrices. The solid matrices used are glucose, trehalose, cyclodextrins, sodium acetate, and filter paper. Heat capacity data are obtained for sodium acetate, sodium acetate/sodium chloride, and cyclodextrin/sodium chloride mixtures over a wide temperature range. These data are used along with the Debye and Einstein models of heat capacities of solids to describe the nonradiative transition from the triplet state to the ground state of the phosphor adsorbed on solid matrices. With filter paper as a solid matrix, infrared data and Young's modulus data from filter paper are related to the moisture quenching of the phosphorescence of organic phosphors adsorbed on filter paper. Room-temperature photophysical data are acquired for several polycyclic aromatic hydrocarbons on different solid matrices and related to the energy-gap law. The correlations developed are used to predict which aromatic hydrocarbons give room-temperature phosphorescence. A new approach is developed for obtaining room-temperature solid-matrix luminescence from lumiphors in solid carbohydrates.

\section{Heavy Element Chemistry}

\section{Florida State University Tallahassee, FL 32306}

\section{Department of Chemistry}

\section{Research in Actinide Chemistry Choppin, G.R. 904-644-3875}

This research emphasizes the basic studies of the behavior in solution of the actinide elements and of the chemically related lanthanide elements. The systems are chosen for investigation because the data can provide increased understanding of the principles governing the chemical behavior of the f-elements with a variety of complexing ligands, both organic and inorganic. The data may also be of direct value for modeling calculations of the behavior of actinides in environmental and waste repository systems or in improved separation schemes of these elements. Emphasis continues on the thermodynamic, kinetic, and 
spectroscopic (absorption and luminescence) studies of the complexation and redox reaction of the actinides. A major environmental ligand studied is humic acid. Binding of actinides in the III through VI oxidation states to humic acid is very rapid. Upon binding, most of the An is "weakly" bound and two days were found to be required to reach equilibrium between "strong" and "weak" binding. Binding studies of $\mathrm{NpO}_{2+}$ in humic acid solutions gave stability constants that had no dependence on $\mathrm{pH}$ in contrast to the behavior of the complexation of An(III), (IV), and (VI), Other systems presently under study involve actinide interaction with silicate ligands. The fluorescent half-life method has provided information on the residual hydration of the trivalent metals in a variety of complexes and in a number of systems used in solvent extraction separations of actinides. Studies on hydrolysis, carbonate, and phosphate complexation are also under way.

\section{University of New Mexico Albuquerque, NM 87131}

\section{Department of Chemistry}

\section{Preorganized and Immobilized Ligands for Metal Ion Separations \\ Paine, R,T. 505-277-1661 \\ $\$ 94,090$}

The objectives of this project are to (1) design new families of organic ligands suitable for selective chelation of f-block element ions present primarily in complex liquid and solid-waste matrices, (2) examine the coordination chemistry and molecular structures of ligands in order to better understand ligand-metal binding, and (3) prepare solidphase, immobilized chelators, The current efforts are specifically directed at the synthesis of noyel phosphonopyridine $\mathrm{N}$-oxide ligands that can display six, seven, and eight-membered chelate rings. These ligands are particularly robust and selective in their metal binding properties. The extraction properties of selected ligands are also characterized by radiochemical methods. Ligands that demonstrate favorable separations properties are then incorporated onto solid organic or inorganic supports, and the steric and electronic factors that subsequently influence the separation properties are examined.

\section{Ohio State University Columbus, OH 43210}

\section{Department of Chemistry}

\section{The Electronic Structure of Heavy-Element Complexes Bursten, B.E. 614-292-1866}

This project focuses on the use of advanced theoretical methods to calculate the electronic structure of organoactinide and related coordination complexes. The principal methodologies currently employed are the fully relativistic discrete variational $\mathrm{X} \alpha(\mathrm{DV}-\mathrm{X} \alpha)$ method, and the ab initio methods with relativistic effective core potentials. A number of interesting new results have resulted from these calculations in the past year. The DV-X $\alpha$ method has been very successful for the calculation of optical excitation energies in $\mathrm{f}^{\mathrm{l}}$ systems, such as the $\operatorname{AnX}_{6}{ }^{\mathrm{q}-}$ complexes ( $A n=P a, q=2 ; \quad A n=U, q=1 ; A n=N p, q=0 ; X=h a l i d e$ ) and more complex $\mathrm{f}^{1}$ organometallic complexes, such as $\left(\eta^{8}-\right.$ $\left.\mathrm{C}_{8} \mathrm{H}_{8}\right)_{2} \mathrm{~Pa}$. Fully relativistic calculations on a series of metal hexacarbonyls, $\mathrm{M}(\mathrm{CO})_{6}$, for $\mathrm{M}=\mathrm{Cr}, \mathrm{W}, \mathrm{U}$, and Sg(element 106), have been completed. These calculations indicate that $\mathrm{Sg}$ behaves as a transition metal with respect to metal-carbonyl bonding, and that, not surprisingly, it is subject to very large relativistic effects. $A b$ initio and density functional calculations have recently been completed on a fascinating new organouranium anion, $\left(\eta^{7}-\mathrm{C}_{7} \mathrm{H}_{7}\right)_{2} \mathrm{U}^{-}$, and a transition metal analog. In the area of theoretical program development, efforts are still underway to develop a quasi-relativistic DV-X $\alpha$ method, one that incorporates all of the major relativistic effects except for spin-obit coupling. This method will allow the results to be presented in the more familiar nomenclature of single groups rather than the double groups required for fully relativistic methods. In another ongoing effort, relativistic effective core potentials for the transactinide elements are being derived.

\section{Chemical Engineering Sciences}

\section{University of California, San Diego La Jolla, CA 92093}

\section{Department of Applied Mechanics and Engineering Sciences}

\section{Premixed Turbulent Combustion Libby, P.A. 619-534:3168}

This project is primarily concerned with flames in stagnating turbulence. Such flames are rich in problems of fundamental interest, e.g., the extinction of turbulent flames. As a consequence, there are five or six laboratories in Western Europe and one in the United States carrying out relevant experimental investigations. We pay close attention to experimental results coming from these laboratories. We have completed and published in Combustion and Flame the first two theoretical studies of these flames utilizing the $\mathrm{k}-\epsilon$ theory of turbulence to characterize the fluid mechanics of the flow. We have also analyzed and compared theory and experiment for nonreactive impinging and counterflowing turbulent streams and have published four journal articles on this work. Although our research is primarily theoretical in nature, a modest experimental effort on turbulent impinging reactant streams was presented at the Twenty-Fifth Symposium (International) on Combustion. An important finding that resulted from an effort to compare existing theory and experiment for flows involving combustion was the realization that heat release vitiates the assumption of a thin flame, the assumption employed in existing theory. Thus an entirely new approach is called for, one treating the entire flow from one jet exit plane to the other or from one exit plane to the wall. An analysis of this flow based on a Reynolds stress theory for the fluid mechanical and the Bray-Moss-Libby theory for the aerothermochemistry has been completed, but preliminary numerical results seem 
to establish that a new representation of the chemical source term is required. This work is ongoing. The study of highly idealized premixed combustion in a channel has been accepted for publication.

\section{Colorado School of Mines Golden, CO 80401}

\section{Department of Chemical Engineering and Petroleum Refining}

\section{Composition of Dependence of Fluid Thermophysical Properties Theory and Modeling \\ Ely, J.F. 303-273-3885 \\ $\$ 115,000$}

The objective of this research is to develop high accuracy predictive models that describe the physical property behavior of complex mixtures. The work is currently progressing along three fronts: (1) development of improved corresponding states models for asymmetric mixtures through reference fluid equation of state design and improved mixing rules; (2) investigation of the phenomenological behavior of the viscosity of molecular (e.g., structured) mixtures through nonequilibrium computer simulation techniques; and (3) development of ultrahigh accuracy equations of state for complex fluids and fluid mixtures through the use of stepwise regression and simulated annealing optimization. The ultimate goal of this research is to develop improved computer-based models for process design that provide accurate predictions of phase transitions and bulk-phase properties in systems that have large size and/or polarity differences.

\section{Colorado State University Fort Collins, CO 80523}

\section{Department of Chemical Engineering}

\section{Study of Improved Methods for Predicting Chemical Equilibria \\ Lenz, T.G. 303-491-7871

This research involves study of computational methods capable of accurate prediction of thermochemical properties. One such method, molecular mechanics (MM), has been found to be a powerful tool for computing the thermodynamic properties of various compounds. Specific recent research with QCFF, a thermodynamic MM program being developed, has involved study of electrostatic energy/charge redistribution capability, and parameterizing QCFF for oxygen, nitrogen, and halogens. The QCFF program has also been modified to accept the input of initial atomic coordinates in various file formats, thus enabling it to be used in tandem with various graphical packages such as CHEMDRAW and BIOGRAF. The enhanced heteroatom capability of the QCFF program was used to compute the heat capacity of R123a $(1,1,1,2-$ tetrafluoroethane), an alternative refrigerant as a replacement for R12 (dichlorodifluoromethane), at $300 \mathrm{~K}$. The computed value for the heat capacity was found to be within $0.5 \mathrm{cal} / \mathrm{mol}$ of the reported experimental value. The
QCFF program was also used to compute the heat capacity of R14lb(1-fluoro 1,1-dichloroethane), which is a potential replacement for R11(trichlorofluoromethane) in foam blowing operations. The computed value differed from the experimental value by $0.57 \mathrm{cal}$ at $300 \mathrm{~K}$. This work thus demonstrates the ability of QCFF to extend the thermochemical database for properties of industrially important compounds.

\section{Cornell University Ithaca, NY 14853}

\section{School of Chemical Engineering}

\section{Simulation of Fluid Behavior in Well-Characterized Porous Materials Gubbins, K.E. 607-255-4385

This work is developing new and rigorous theoretical and simulation methods for the study of the adsorption behavior of fluids in well-characterized porous materials. In particular, fluids in porous carbons, aluminosilicates (particularly MCM-41), aluminophosphates, and pillared clays are being studied. The work may later be extended to other well-characterized materials. Studies in the first stage of the project have been on adsorption, heat of adsorption, phase changes, hysteresis, and diffusion for simple fluids (inert gases, methane, nitrogen) and their mixtures. Fluids of chain molecules (both alkanes and simple models of associating chain molecules) in carbons are also under study. As a second stage of the work, studies are under way on the behavior of water in carbons and aluminophosphates, which is quite different from that of the simple gases. The project includes experimental studies of adsorption in some of these materials to provide data to test the models.

\section{Molecular Simulation of Phase Equilibria for Complex Fluids}

Panagiotopoulos, A.Z. 607-255-8243

The objective of this project is the investigation of phase equilibria for complex fluids using molecular simulation techniques. Current research encompasses three main classes of systems, namely polymeric, surfactant, and ionic systems. We have recently developed a novel algorithm for constant-pressure and Gibbs ensemble simulations of lattice models and have used it to test theoretical models for the phase behavior of simple homopolymers and nonionic model surfactants. We have also studied binary polymer/solvent mixtures and evaluated engineering models and molecular theories against simulation results for the chemical potential and volumetric properties. Improved theoretical models in the framework of generalized Flory dimer theory were developed as a result of our work in this area. For ionic systems, we have started exploring a promising new class of "thermodynamic scaling" Monte Carlo algorithms that allow efficient determination of free energies of strongly interacting systems. We have used these methods to obtain the phase behavior and location of conductor/insulator transition for three- and two-dimensional coulombic systems. 
Department of Mechanical and Aerospace Engineering

\section{Reaction and Diffusion in Turbulent Combustion \\ Pope, S.B. 607-255-4314}

Direct numerical simulations (DNS) are being used to study fundamental processes in non-premixed turbulent reacting flows. A broad range of Damkohler number, $\mathrm{Da}$, and reaction zone thicknesses are being studied, encompassing stable, near-equilibrium reaction; local extinction; and global extinction. A simple thermochemical model for one-step reversible reaction is employed, in which the state of the fluid is described by the mixture fraction $s(\mathrm{x}, \mathrm{t})$ and the perturbation from equilibrium $y(x, t)$. A pseudospectral method, with grid sizes up to $128^{3}$, is used to solve the Navier-Stokes equations and the conservation equations for $\varsigma$ and $y$. These equations are augmented with artificial forcing, so that the resulting velocity and mixture fraction fields are statistically stationary, homogeneous, and isotropic. At sufficiently high Damkohler number, the perturbation field is also statistically stationary, corresponding to stable reaction. But at lower $\mathrm{Da}, \mathrm{y}$ increases without bound, corresponding to global extinction. The critical Damkohler number is Dacrit at which global extinction occurs, has been determined from the simulations, and is found to be significantly different from that predicted by simple models (flamelet, conditional moment closure, etc.). Currently the DNS code is being extended to run on parallel processors allowing for larger grid sizes; and two other flows-the reactive seals mixing layer, and periodic reaction zones-are being considered.

\section{University of Delaware Newark, DE 19716}

\section{Department of Chemical Engineering}

\section{New Generation of Group Contribution and Equation-of-State Models \\ Sandler, S.I. 302-831-2945 \\ $\$ 112,000$}

To make intelligent, cost-effective decisions for the design of new processes, for the development of synthetic fuels and other new technologies, and for estimating the environmental fate and bioaccumulation of chemicals, accurate methods of predicting and correlating thermodynamic properties and phase equilibria are needed. The research being conducted under this grant addresses this problem from three different directions. First, the recently developed Wong-Sandler mixing rules, which have greatly expanded the range of application of equations of state to highly nonideal mixtures, are being studied for collections of mixtures that previously could not be accurately described over large ranges of temperature and pressure. This includes hydrogen-containing mixtures and mixtures with strongly polar compounds. The second area of research is the use of ab initio molecular orbital calculations to compute the effect of hydrogen-bonding and other strong association phenomena. The results of these calculations are being incorporated into a new generation of group contribution methods (such as UNIFAC) to improve the accuracy of these important prediction methods. The third area of research is to use computer simulation and statistical mechanical theory to develop an accurate thermodynamic description of long chain hydrocarbons, polymers, and mixtures involving these components. The Wong-Sandler mixing rules, discussed above to study solutions of these chain components in small molecule solvents, are also being used.

\section{University of Illinois at Chicago Chicago, IL 60680}

\section{Department of Chemical Engineering}

\section{Transport Properties of Dense Fluid Mixtures Using Nonequilibrium Molecular Dynamics \\ Murad, $S$. 312-996-5593}

Computer simulation studies have been carried out to study the phenomena of osmosis and reverse osmosis in solutions. These studies have been carried out using both a molecular dynamics method recently developed by us, as well as the Gibbs ensemble MonteCarlo (GEMC) method. Both gas mixtures and liquid (including aqueous) solutions are being investigated, since reverse osmosis is considered an attractive technique for separations in these systems. Our results show that computer simulations could prove to be a useful tool determining the feasibility of reverse osmosis as a possible technique for many industrial separations problems. Computer simulations using the method of nonequilibrium molecular dynamics have been carried out to study the enhancement in thermal conductivity of simple fluids near the critical region. Our results show that for system sizes larger than 10 molecular diameters, critical effects begin to be observed. We plan to begin studying mixtures as well as polyatomic systems shortly.

\section{Johns Hopkins University Baltimore, MD 21218}

\section{Department of Chemical Engineering}

\section{Prediction of Thermodynamic Properties of Coal Derivatives \\ Donohue, M.D. 410-516-7761 \\ $\$ 85,000$}

Changes in the chemical and petrochemical industries have led to the need to predict properties for substances and mixtures that are complex in their chemical structures and their intermolecular potential functions. This project uses a combination of experiments, computer simulations, and theory to better understand and describe these systems. The focus of current effort is in developing more accurate models to predict the properties of spherical and long-chain molecules that interact with model potential energy functions. It is hoped that by improving the accuracy of model-system predictions, we will be able to provide better explanations and predictions for several poorly understood or poorly described pheonomena. Examples where such fundamental theoretical improvements would be of great benefit include the prediction of dew points in natural-gas mixtures and nonrandomness 
effects ("clustering") in süpercritical fiuids. Our experimental program includes spectroscopic measurements of specific molecular interactions '(e.g., hydrogen bonding) as well as nonspecific interactions (Liondon dispersion forces) to probe nonrandom and density-dependent behavior.

\section{University of Maryland at College Park College Park, MD 20742}

\section{Institute for Physical Science and Technology}

\section{Thermophysïcal Properties of Fluids and . Fluid Mixtures \\ Sengers, J.V. 301-405-4805

It has become 'evident that critical fluctuations affect the thermodynamic and transport properties of fluids and fluid mixtures in a wide range of temperatures and densities. Equations that incorporate the effects of these critical fluctuations on the thermodynamic ard transport properties are being developed. The procedure is being used to develop accurate equations and tables for a number 'of importan't fluids including alternative refrigerants. The theory has also been extended to develop equations for the thermodynamic properties and transport properties of fluid mixtures like mixtures'of carbon dioxide and ethane that have a Type I phase diagram with a continuous critical line. We are in the process of extending the theory to fluid mixtures that have more complex phase diagrams. The theory is also being applied to renormalize practical chemical engineering equations of state for the effects of critical fluctuations.

\section{University of Massachusetts at Amherst Amherst, MA 01003}

\section{Department of Chemical Engineering}

\section{Theoretical Studies of Solid-Fluid Phase Equilibria \\ Monson, P.A. \\ -413-545-0661 \\ $\$ 109,624$}

This project focises on the application of statistical thermodynamics to the calculation of solid-fluid phase equilibrium. We have recently completed a study of the combined influence of molecular shape and quadrupolar -interactions upon solid-fluid equilibrium using Monte Carlo simulation and theory. This work has led to an improved understanding of the origin for the relatively small region of liquid state stability for linear molecules with high quadrupole moments, such as carbon dioxide and acetylene. A study of solid-fluid equilibrium for flexible chain molecules has also'been initiated. The results of this work will be used to analyze the solid-fluid equilibrium in chain molecules, such as the normal alkanes, from a corresponding states perspective, and to develop generalized van der Waals theories of solid-fluid equilibrium. We have developed a theory for the properties of substitutionally ordered solid solutions (or "compounds") of hard spheres.
This theory, which extends a theory for solid solutions developed in our previous work, correctly describes how packing 'effects can create substitutional order in the solid phase. The theory is currently being extended to the treatment of attractive intermolecular interactions.

\section{National Institute of Standards and Technology, Boulder Boulder, CO 80303}

\section{Thermophysics Divison}

\section{Integrated Theoretical and Experimental Study of the Thermophysical Properties of Fluid Mixtures: Properties of Mixtures Near a Phase Transition Hanley, H.J.M.; Friend, D:G. $303-497-3320$

The research includes three major'components: scattering studies of complex fluid systems; theory and computer simulation studies of the solid-fluid transition; and study of phase behavior and properties in the critical region of mixtures. The major objective of the work is to develop and refine theoretically based predictive models for the thermophysical properties of complex fluid mixtures. Scattering studies have been performed on binary suspensions of polystyrene and silica in contrast-matched solvents, and studies of suspensions under shear have shown the associated distortion of the fluid structure factors. The experimental equilibrium structure factors complement theoretical and simulation work on heterogeneous phases using ideas from density functional theory. In nonequilibrium molecular dynamics studies, a new thermostatting technique has been developed, and shear-induced melting has been examined. A computer code to describe fluidphase equilibria in Type II fluid mixtures has been developed according to nonclassical, scaling precepts. Classical extended corresponding states models have been used to examine the critical region behavior of Type III aqueous systems. Work is continuing in the ongoing effort to understand and predict the thermophysical properties of complex mixtures.

\section{North Carolina State University Raleigh, NC 27695}

\section{Department of Chemical Engineering}

\section{Theoretical Treatment of the Bulk and Surface.Properties of Fluids Containing Long, Flexible Molecules \\ Hall, C.K. \\ 919-515-3571 \\ $\$ 102,000$}

This research program is designed to enhance understanding of the behavior of fluids and fluid mixtures containing chain-like molecules. The objective is to develop a theory that is capable of predicting the experimentally observed thermophysical properties, including phase equilibria and transport properties, of fluids and fluid mixtures containing chain-like molecules ranging in 'length from alkanes to polymers. This year the Generalized Flory dimer theory has been extended to (1) binary 
mixtures containing hard chain molecules that differ in both segment size and well depth, (2) heteronuclear hard chains, and (3) fused hard sphere chain molecules. Work has begun on extracting activity coefficients from the Generalized Flory Dimer theories, with the aim being to use this as a basis for developing a new group contribution approach to estimate the properties of mixtures when experimental data are unavailable. The most extensive computer simulation study to date on the dynamics of fluids containing very long $(n=192)$, entangled polymers has also been completed. The results reveal the presence of a heretofore unobserved scaling regime associated with the presence of localized knots. This could be the molecular origin of discrepancies between theoretical predictions and experimental observations of the molecular weight dependence of shear viscosity.

\section{University of Pennsylvania Philadelphia, PA 19104}

\section{Department of Chemical Engineering}

\section{Molecular and Polymeric Fluids in Microporous Media Glandt, E.D. 215-898-6928}

This is an investigation of the effect of confinement on both molecular and macromolecular species into micropores. Integral equation theories are combined with moleculan simulations to predict the structure and the thermodynamic properties of the solute species. Both equilibrium and rate-limited separation processes are strongly affected by the types of phase coexistence investigated here. The work is investigating the applications of polymer RISM theory to the determination of the intermolecular structure of confined linear chains: A comparison among the several models that have been proposed for the intramolecular correlation function suggests the most appropriate form for this choice. An important outstanding problem remains: that of predicting the effective size (e.g., the radius of gyration) of a polymer chain when it is transferred from a bulk phase to the pores of a solid matrix. A self-consistent approach appears as the most promising route for this prediction. The computed results agree very well with our own molecular simulations. Furthermore, the numerical results for the partition coefficient of polystyrene dissolved in chloroform into microporous glass agree rather well with experimental information for this system.

\section{Princeton University Princeton, NJ 08544}

\section{Department of Chemical Engineering}

417. Computational and Theoretical Studies of Homogeneous Nucleation in Supercritical Fluids

Debenedetti, P.G.

$\$ 89,827$

609-258-5480

Two new computational techniques have been developed to calculate free energies of nucleation in on-ideal fluids and fluid mixtures. They are being used to calculate freeenergy barriers to crystal nucleation in supercooled. liquids and supersaturated solutions. A lattice model of a network-forming fluid with orientational bonding has been developed to study the thermodynamics of highly supercooled water. Significantly, it reproduces all of the scenarios that have been proposed to date to explain the anomalous properties of supercooled water. This strongly suggests that what had previously been regarded as mutually exclusive interpretations are, in fact, closely related and that similar behavior may occur in other networkforming fluids, such as silica and germania. The thermophysical properties of highly superheated model liquids and supercooled vapors have been studied by computer simulation. The technique involves imposing constraints that prevent incipient phase separation. Constraints explored so far include restricting density fluctuations and, for superheated liquids, limiting the size of cavities that are allowed to form. The equation of state is sensitive to the severity of void constraints and much less so to restrictions on density fluctuations.

\section{Stanford University Stanford, CA 94305.}

\section{Department of Chemical Engineering}

\section{Findamental Studies of Fluid Mechanics and Stability in Porous Media \\ Homsy, G.M. 415-723-2419 \\ $\$ 80,000$}

This research project treats flow and transport problems in porous media, which are of interest in energy recovery processes. Both macroscopic Darcy scale and pore scale flows: are studied. In the former case, fingering instabilities in homogeneous media are studied for conditions under which both gravitational and viscous forces are present and can be both destabilizing, both stabilizing, or can compete against one another. In the last case we find surprising similarities with 'reverse fingering', associated with nonmonotonic mobility profiles discovered earlier in our group. Current work is focused on the interactions between fingering instabilities and flows driven by permeability heterogeneity. Current pore-scale work focuses on the propagation of interfaces of wetting and nonwetting viscoelastic fluids. Theory shows that the instability of interface propagation depends on contact angle dynamics, capillary number, and Weissenberg number. Perturbation theory for small Weissenberg numbers has shown how viscoelastic thin-film dynamics differs from the Newtonian case. In the wetting case, the hydrodynamically entrained film is thinner due to the increased resistance to elongational flow, and viscoelastic forces destabilize the interface. In the nonwetting case of the viscoelastic moving contact line, normal stresses act to enhance the spatial variation of film thickness, again leading to increased instability. 


\section{State University of New York at Stony Brook Stony Brook, NY 11794}

\section{Department of Chemistry}

\section{Thermophysical Properties of Fluids and Fluid Mixtures \\ Stell, G.R. 516-632-7899 \\ $\$ 118,000$}

Theoretical research continues on the thermophysical properties of fluids using a statistical mechanics-based approach to do molecular modelling. Current emphasis is on the study of phase separation in ionic fluids, on fluids of associating particles, and on fluids in porous media. The work on ionic fluids includes studies in which the assumptions of the mean spherical approximation are augmented by new treatments of ionic association to yield phase diagrams in which the location of phase separation is more accurately predicted. The work on association includes studies of dimerization and polymerization in model liquids of reacting atoms in which the core volume of the product molecules is considerably smaller than the sum of the core volumes of the reacting monatomic particles. The work on fluids in porous media includes a general study of thermodynamic relations for a simple fluid in such a medium that is assumed to have a realistically irregular pore structure, in contrast to earlier studies of fluids in single pores of a particular shape.

\section{University of Tennessee at Knoxville Knoxville, TN 37996}

\section{Department of Chemical Engineering}

\section{Theoretical and Experimental Study of Mixed Solvent Electrolyte Systems Cummings, P.T. 615-974-0227

The objective of this project is to develop fundamentally based predictive theories for the thermodynamic properties and phase equilibria in mixed solvent electrolyte systems. The emphases in the current year are molecular simulation of vapor-liquid phase equilibria in methanol/ water and ethanol/water/salt systems using a new water potential, molecular simulation of supercritical aqueous systems, and additional experimental measurements of phase equilibria and densities in alcohol/water/organic salt systems.

\section{Yale University \\ New Haven, CT 06520}

\section{Department of Mechanical Engineering}

\section{Computational and Experimental Study of Laminar Premixed and Diffusion Flames}

Smooke, M.D.; Long, M.B.

$\$ 200,000$ 203-432-4344

Our research centers on an investigation of the effects of complex chemistry and detailed transport on the structure and extinction of hydrocarbon flames in coflowing axisymmetric configurations. We have pursued both computational and experimental aspects of the research in parallel. The computational work has focused on the application of accurate and efficient numerical methods for the solution of the boundary value problems describing the various reacting systems. Both serial and parallel (shared and distributed memory) computations have been performed. Detailed experimental measurements were performed on axisymmetric coflow flames using two-dimensional imaging techniques. In particular, spontaneous Raman scattering and laser-induced fluorescence were used to measure the temperature, major and minor $(\mathrm{OH}$ and $\mathrm{CH})$ species profiles. Our goal during this period has been to obtain a more fundamental understanding of the important fluid dynamic and chemical interactions in these flames so that this information can be used effectively in combustion modeling.

\section{Advanced Batteries}

\section{Arizona State University Tempe, AZ 85287}

\section{Department of Chemistry}

\section{Application of New Concepts in Solid Electrolytes, and Pressure-Stabilized Cathode Materials, to Solid State Battery Technology \\ Angell, C.A. \\ 602-965-7217

This research represents the second stage follow-up of a new class of electrolytes, the so-called polymer-in-salt or ionic rubber, electrolytes. Emphasis has shifted from boron-centered anions and complex sulphonate anions which, surprisingly, prove not to be low-melting, to various modifications of the sulfonimide anions, of which the well-known case is trifluoromethanesulfonimide. Some of the new imide salts completely fail to crystallize. While with one exception their conductivities tend to be low in the pure state because of poor decoupling ratios, their complexes with Lewis acids like $\mathrm{AlCl}_{3}$ are high-conducting and electrochemically stable. For reasons still incompletely understood, $\mathrm{Al}$ is not deposited in cyclic voltammograms of these salts. On the other hand, $\mathrm{Li}$ is reversibly deposited and stripped. Cells constructed with lithium anodes and $\mathrm{Li}$ manganate cathodes deliver 3.5-3.8 
$\mathrm{V}$ and are rechargeable over a number of cycles. Evaluation of interface impedances and general optimization of such cells is in progress. Anionic polymers and oligomers of a novel variety are being tested for use with these electrolytes to provide rubbery properties.

\section{California Institute of Technology Pasadena, CA 91125}

\section{Department of Materials Science}

\section{Design of Metal Hydride Alloys for Battery Electrodes with High Cycle Lifetimes}

Fultz, B.; Ratnakumar, B.V. $\$ 175,923$ 818-395-2170

This program will design new alloys for service as negative electrodes in nickel metal hydride battery cells. The two goals are: (1) to improve the cycle lifetime during charge/ discharge cycling, and (2) to understand the mechanisms controlling the lifetimes of metal hydride electrodes. Ternary solutes are being added to $\mathrm{LaNi}_{5}$ in a systematic way and in dilute amounts so that chemical trends can be determined. Studies have begun on the temperature dependence of the cyclic lifetime of the metal hydride electrodes, both during electrochemical cycling and during gas-phase cycling. Activation energies measured from these temperature-dependent experiments should provide an understanding of the mechanisms that cause deterioration of the hydrogen storage capacity of the materials, especially in combination with our microstructural and electrochemical characterizations of these materials.

\section{Clark University Worcester, MA 01610}

\section{Department of Chemistry}

\section{Novel Aluminum and Sulfur Batteries Licht, $S$. 508-793-7112

Work is actively proceeding on development of a new battery, the aluminum sulfur battery. Each storage cell contains an aluminum anode, a solid sulfur, and/or aqueous polysulfide cathode and operates at or near room temperatures. Both the very high discharge domain (over $10 \mathrm{~mA} / \mathrm{cm}^{2}$ ) and the low discharge domain (less than 0.1 $\mathrm{mA} / \mathrm{cm}^{2}$ ) of the cell are being studied and optimized. Development includes optimization of the aluminum anode and of the sulfur cathode through electrochemical, spectroscopic, and potentiometric measurements in a variety of temperature, concentration, and partial discharge domains for the combined solid sulfur-aqueous polysulfide system. Measurements of aluminate, hydroxide, and solution additive concentrations during anode discharge are being studied to improve anodic utilization efficiencies under a variety of discharge conditions. Cells incorporating the most promising modifications are being tested. From these discharge tests, high energy capacity cells will be designed and tested with a $250-\mathrm{Wh} / \mathrm{kg}$ objective.

\section{University of Minnesota Minneapolis, MN 55455}

\section{Corrosion Research Center}

\author{
425. Characterization of Insertion Electrodes \\ in High-Energy Cell \\ Smyrl, W.H. \\ 612-625-0717 \\ $\$ 275,625$
}

The research program has three basic thrusts. In the first, the intercalation of $\mathrm{Li}$ into carbonaceous is materials is investigated. Researchers have used highly oriented pyrolytic graphites (HOPG) with etched, single atomic layer "pits" as well as defined sites for intercalation of $\mathrm{Li}$ which was deposited from the vapor phase. STM measurements were used to characterize the morphology of the surface before and after the exposure of $\mathrm{Li}$ vapor. A ring of intercalated $\mathrm{Li}$ was formed just inside the pit edge, under the top layer of graphite. The $\mathrm{Li}$ moved away from the initial sites in channels that were probably controlled by residual stress in the HOPG. Further work has shown that intercalation from the liquid phase into HOPG can be accompanied by other processes that disrupt the insertion process, and cause degradation of the substrate. Transmission electron microscopy has shown that severe exfoliation and solvent intercalation occurs in $\mathrm{LiC10}_{4} /$ propylene carbonate solutions that are used in lithium battery applications. This is the first direct evidence of exfoliation that has been found, although it had been suspected previously. The exfoliation is completely absent in other electrolytic solutions, namely LiPF 5 /ethylene carbonate-diethylene carbonate solutions. The reasons for the differences are not understood at present. Additional work is under way to synthesize novel forms of carbon that will have high capacities for $\mathrm{Li}$ insertion. $\mathrm{Li}$ :C ratios of 1:2 have been obtained (much higher than the 1:6 ratio believed to be the limit previously), but it has not been possible to retain the high capacity on repeated insertionrelease cycles. In the second basic thrust, the intercalation host, $\mathrm{V}_{2} \mathrm{O}_{5}$ xerogel, has been studied to establish the physical and chemical properties that may be manipulated by sol-gel processing. Vanadium pentoxide films were spincoated on nickel/silicon substrates and characterized by XRD, SEM, TEM, and optical and electronic conductivity measurements. A largely amorphous film was obtained by spin coating at high rotation speeds, with the layered microstructure becoming more dominant as the rotation speed is reduced. The electronic conductivity of the films are increased as the amorphous character is increased. Further, the conductivity in the plane of the film is three orders of magnitude larger than the conductivity normal to the surface for highly layered films (consistent with the expectation that transport along the plane of the layers is larger), but the difference is less than two orders of magnitude in amorphous films. The third thrust is to establish the capability of the films to host polyvalent cations in addition to Li. It has been established that a chemical insertion technique can be used to survey different metal cations. The technique consists of reacting a solution of the iodide of the metal of interest with vanadium pentoxide xerogel films. As $\mathrm{V}_{2} \mathrm{O}_{5}$ xerogel films oxidize iodide (the film being reduced at the same time), the metal 


\section{School of Physics and Astronomy}

\section{Modeling of Transport in Lithium Polymer Electrolytes for Battery Applications Halley, J.W. 612-624-0395 \\ $\$ 100,302$}

The goal of this research is to provide new insight into the mechanism of ionic conduction in $\mathrm{Li}$ polymer electrolytes and the electrolyte-electrode interface using a combination of new and powerful simulation techniques that have not previously been applied to these materials. A molecular dynamics model for amorphous polyethylene oxide has been developed, using a unique approach in which the model is developed by simulating a polymerization process, starting with liquid dimethyl ether. This has significant advantages over starting a molecular dynamics simulation in the crystalline phase. We are using the molecular dynamics model to evaluate solvation and hopping activation free energies for $\mathrm{Li}^{+}$ions and various cations. This output from the molecular dynamics studies will be used to construct lattice models of the system at larger length and longer time scales appropriate to the transport problem and to evaluate various suggestions concerning the nature of the ionic transport in these electrolytes. Later, the models will also be used to study the electrode-electrolyte interface.

\section{Moltech Corporation Stony Brook, NY 11794}

\author{
427. New Materials Technology For \\ Rechargeable Lithium Batteries \\ Skotheim, $T$. \\ 516-444-8865
}

$\$ 100,000$

Rechargeable batteries with high specific energy are of critical importance for applications where portability is an issue. The objective of this program is to develop significantly improved polymer technology that will be incorporated into thin film rechargeable lithium cells, which have the potential to leapfrog existing technologies. We are currently developing advanced battery technologies and materials for high-capacity, long-cycle-life lithium polymer batteries. Our work on high-capacity cathode materials, high-conductivity polymer electrolytes, and high-stability anodes has gained significant interfaces progress, including synthesis and characterization of all materials made in-house. Processing techniques for cathode and polymer electrolytes are determined and/or improved, resulting in better performance of our laboratory test cells.

\section{University of Nevada at Las Vegas Las Vegas, NV 89154}

\section{Department of Physics}

\section{Long-Ranged Polymer Dynamic Behavior and Conductivity in Battery Polymer Electrolytes: Poly(ethylene oxide)/Salt Systems \\ Selser, J.C. \\ 702-895-3847 \\ $\$ 20,000$}

The most compelling application driving the intense research into the properties and uses of polymer electrolytes is the development of secondary (rechargeable) lithium batteries. The work proposed here will use light scattering, principally the noninvasive dynamic light scattering technique known as photon correlation spectroscopy, or "PCS", to study long-ranged, slow relaxations in poly(ethylene oxide) (PEO). Polymer hosts are generally acknowledged as among the "top choices" for polymer electrolytes. PEO itself and PEO-based polymer electrolytes are used both for research and for application. The purpose of this work will be to determine, under the same conditions, the relationship between conductivity in PEO electrolytes and long-ranged PEO relaxations in PEO electrolytes, as probed by light scattering. In the first part of the proposed work, unique expertise acquired in this laboratory will be applied to the study of the salt concentration dependence of the slowest, overall, intracoil PEO relaxation in dilute methanol solutions. In this way, the effects of increasing salt concentration on a single, well-defined intrapolymer relaxation will be determined. In the second part of the work, pure PEO melts and PEO electrolyte melts containing stoichiometric amounts of selected salts will be investigated. In particular, the salt concentration and temperature dependencies of long-ranged, slow PEO relaxations in PEO electrolytes will be studied. Comparisons of the results from the PEO solution study and the PEO electrolyte study along with a comparison of the PEO electrolyte results with results from studies of the effects of salt concentration and temperature on PEO conductivity will lead to a better understanding of how long-ranged $\mathrm{PEO}$ relaxations in PEO electrolytes affect conductivity in these electrolyte systems.

\section{North Carolina State University Raleigh, NC 27695}

\section{Department of Chemical Engineering}

\section{Composite Polymer Electrolytes Using Cross-Linked Fumed Silica Fillers in Low Molecular Weight Polyethylene Oxides: Synthesis, Rheology and Electrochemistry Khan, S.A.; Fedkiw, P.S.; Baker, G.L. 919-515-4519 \\ $\$ 141,378$}

The objective of this project is the synthesis and evaluation of a composite polymer electrolyte system for use in rechargeable lithium batteries. Based on surface-modified, cross-linked fumed silica and low molecular weight polyethylene oxide/glycols, this new approach should yield low-cost, processable solid electrolytes with conductivities 
that rival gel electrolytes. The principal features of the new system include: (i) exceptional processability; the composite is processable as a viscous fluid using standard techniques but can set to give a solid with a yield point, (ii) dimensional stability; the fumed silica can be cross-linked by UV, thermal, or other curing methods to improve the mechanical properties and ensure dimensional stability, (iii) good conductivity; room temperature conductivities for electrolytes containing lithium salts should easily reach $5 \times 10^{-4} \mathrm{~S} / \mathrm{cm}$, (iv) low cost; this is a consequence of simple processing and the ready accessibility of the materials, and (v) inherent safety; cured composite electrolytes should have the electrochemical stability typical of solid polymers. Current efforts have focused on the synthesis of surface-modified fumed silica and understanding the processing/rheological behavior of fumed silica composites. Two different types of surface-anchored groups have been attached to commercial fumed silica; these materials are being characterized to determine and optimize their surface coverage. The surface-anchored groups will enable modulating the aggregation pattern of fumed silicas and their compatibility with polyethylene oxide. Rheological studies have been undertaken on commercial fumed silica systems to determine the effect of processing on these systems. Initial studies indicate that shear induces structural breakdown in these systems; however, the structure reforms instantaneously upon cessation of shear. The extent of structural recovery is a function of shear; exposure to low shear leads to a lower modulus materials whereas high shear causes the material to achieve its initial modulus. The instantaneous recovery of the material and to its initial level following large shear are extremely useful features for effectively processing these composites.

\section{Pennsylvania State University, University Park University Park, PA 16802}

\section{Center for Advanced Materials}

\section{Development of Novel Strategies for Enhancing the Cycle Life of Lithium Solid Polymer Electrolyte Batteries Macdonald, D.D. 814-863-7772

The objective of this research is to define a lithium solid polymer electrolyte intercalation cathode ( $\mathrm{Li} / \mathrm{SPE} / \mathrm{IC}$ ) battery prototype with improved cycle life through a better understanding of the kinetics and mechanisms of capacity degradation. This will be done by the collaborative interactions of a research group experienced in the design and synthesis of solid polymer electrolytes of polyphosphazenes and a research group experienced in the engineering and electrochemistry of battery systems. The selection and optimization of electrode and polymer electrolyte materials includes developing intercalation cathodes based on $\mathrm{V}_{6} \mathrm{O}_{13}$ and solid polymer electrolytes based on polyphosphazenes and optimizing their characteristics. Studies of mechanical degradation caused by battery cycling will be estimated by measuring the expansion and contraction of various cells upon charge and discharge. Electrochemical impedance spectroscopy (EIS) is used to investigate the physico-electrochemical processes such as interface and electrolyte impedance changes and intercalation cathode degradation. Destructive analysis of cell components after cycling due to mechanical (dendrite formation) and thermal (reaction products at localized hot spots) mechanisms will be studied using a variety of diagnostic techniques. Battery system models will be developed to guide new battery development and reduce the effort involved in designing by experiment. Enhancing cycle life in Li/SPE/TC batteries will be pursued by examining the degradation mechanisms elucidated in this research.

\section{Rútgers, The State Üniverssity of New Jeirsey Piscataway, NJ 08855}

\section{Cóllege of Engineering}

\section{Solid Ëlectrolyte-Electrode Interfaces: Átomistic Behavior Analyzed Via UHV-AFM, Surfacé Spectroscopies, and Computer Simulations \\ Garofalini; S.H. 908-445-2216

ÜHV=ȦFM, STM, and XPS sürace analysès and molecular dynamics computer simulations have been used to examine the structural and dynamic properties of the electrode/electrolyte interface in solid-state oxide thin-film batteries. In the experiments, the electrolytes have in cluded lithium-containing borates, phosphates, and phosphorous oxynitride ion conducting (IC) glasses and $\mathrm{Li}$ and $\mathrm{V}_{2} \mathrm{O}_{5}$ as the electrode materials. Lithium silicates and $\mathrm{WO}_{3}$ and $\mathrm{V}_{2} \mathrm{O}_{5}$ have been studied in the simulations. Lithium migration into the oxide electrode during formation of the interface between the electrode and the IC oxide glasses has been evaluated using XPS and the simulations. Results show significant lithium migration across the interface during interface formation in both the experiments and the simulations. In the XPS studies, $\mathrm{V}_{2} \mathrm{O}_{5}$ was deposited in situ onto lithium borate, lithium phosphate, and lithium phosphorouis oxy-nitride glass substrates using RF sputtering. The thickness of the IC glass substrates was varied from $50 \AA$ to $750 \AA$. The Li concentration in the electrode was measured as a function of electrode overlayer thickness on each glass substrate. A correlation between substrate thickness and $\mathrm{Li}$ penetration into the electrode was observed. While the simulations of the silicates follow previous work with these systems, new potentials have been generated that give stable crystalline forms of vanadia and tungsten oxide and the lithiated versions of each. Molecular dynamics simulations show penetration of $\mathrm{Li}$ from the IC glass into the electrode, although the behavior differs as a function of crystal orientation. 


\section{Texas A \& M University College Station, TX 77843}

\section{Center for Electrochemical Systems and Hydrogen Research}

\section{Cell Components with Emphasis on Hydride Electrolytes for NickellMetal Hydride Batteries \\ Srinivasan, $S$. 409-845-8281}

The objectives of this work are to tailor-make and evaluate promising alloys for hydride electrodes. The investigations include the elucidation of the role of individual elements on the thermodynamics and kinetics of hydriding/dehydriding and the optimization of the composition and structure of the metal hydride electrodes. The project involves the collaboration of investigators at Texas A\&M University, Brookhaven National Laboratory, Hughes Aircraft Corp., and Los Alamos National Laboratory. Ce and Co substitutions in $\mathrm{LaNi}_{5}$ alloys result in an improvement in the cycle life of the alloys. Most of the Sn substituted compounds exhibit high hydriding capacities. From a fundamental point of view, it is necessary to gain insight on the electrode kinetics of hydriding/dehydriding reaction. There was good agreement between the values of the electrode kinetic parameters, as obtained by Electrochemical Impedance Spectroscopy (EIS), and a method involving discharging the electrode at different rates. These experiments revealed that the rate-determining step in the hydriding reaction is the charge transfer process. From XANES results, the kinetics of $\mathrm{MH}$ electrode reaction could be explained by the decreasing number of $\mathrm{Ni} d$ band vacancies caused by substitution for $\mathrm{Ni}$ with $\mathrm{Sn}$. The microcalorimetric technique showed that the ratedetermining step for the gas phase hydriding/dehydriding reaction is that of diffusion of atomic hydrogen into and out of the alloy. The corrosion behavior of $\mathrm{AB}_{5}$ metal hydride electrodes, investigated using the scanning vibrating electrode techniques (SVET), revealed that the reduced corrosion current in the Ce-containing electrode is consistent with previous results, which showed that $\mathrm{Ce}$ substitution for $\mathrm{La}$ in the $\mathrm{AB}_{5}$ alloy has a beneficial effect on electrode lifetime.

\section{Small Business Innovation Research}

The following projects were funded by the Small Business Innovation Research (SBIR) Program Office in response to a topic submitted by the Division of Chemical Sciences to the annual SBIR solicitation.

The Phase I projects are funded for 6-1/2 months and are designed to evaluate the merits and feasibility of the ideas proposed. Proposals are considered as the result of a solicitation, and awards are made after a competitive evaluation.

The Phase II projects are funded for two years and constitute the principal research and development effort aimed at proving the feasibility of the concept of carrying the process or product to the point where it can be commercialized. They are continuations of Phase I projects. Again, awards are made on the basis of competitive evaluation. Summaries of new Phase I projects as well as Phase II projects are listed.

\section{ANDCARE, Inc. Durham, NC 27713}

\section{An Advanced Electrochemical Sensor for the Simultaneous Analytical Measurement Wojciechowski, $M$. 919-544-8220 $\$ 750,000$}

The requirements of complex sample monitoring present a growing need for a new generation of sensors with small size and multianalyte capability. The hypothesis that screen printing of basic sensor components and circuitry followed by precision deposition of colloidal gold enzyme conjugates and other analytical reagents onto the surface of working electrodes can yield high performance, disposable sensors with many applications is the basis of this project. Phase I research and development showed the feasibility of using electrochemical sensors and monitors for the determination of multiple chemical species, which makes possible the development of new instrumentation that is needed for process monitoring, environmental testing, and medical diagnostics. Phase II research will continue the sensor/monitor development begun in Phase I and produce manufacturing prototypes for the first products. The Phase II goal is to further develop key principles of multianalyte sensor design, operation, and manufacture that set the stage for the broadest possible range of operating capabilities. Phase II will develop the first in a line of needed commercial products with sensors for simultaneous measurement of total cholesterol, high density lipoproteins (HDL) and triglycerides. This multianalyte monitoring approach eliminates the time-consuming care of reusable sensors because the inexpensive manufacture of printed sensor electrodes allows for use of factory-calibrated disposable sensors whose output is coupled to an inexpensive, hand-held monitor

\section{Analysis Consultants Forest, CA 92630}

\section{A New Type of Acoustical Sensor for Chemical Measurements Martin, B.G. 714-803-1033}

This project involves an entirely new sensor concept for monitoring the presence, concentration, and reactions of a wide variety of chemical species, including organic solvents, salts, and biological chemicals. This sensor design exploits the acoustical properties of symmetric multilayers placed on a piezoelectric transducer. These properties are extremely sensitive to changes in layer density, thickness, and elastic constants, all of which are functions of the molecular state. The symmetric multilayer materials used would be a class of bioelastic polymers which are 
capable of converting many forms of chemical reaction energy into changes affecting the molecular state. The major advantages of the planned sensor are its sensitivity, versatility, and chemical specificity. The Phase I effort will be to design, fabricate, and test a prototype sensor utilizing bioelastic polymeric materials. This would involve the selection of a polymer for a specific chemical reaction, e.g., one that is $\mathrm{pH}$ sensitive, or one that is sensitive to the presence of a salt or an organic solvent.

\section{Compact Membrane Systems, Inc Wilmington, DE 19803}

\section{Chemically Resistant Gas Separation Perfluoromembranes \\ Neaser, $S$. 610-499-8860}

Current membranes for gas separation are based on organic polymerics. These organic polymers have significant temperature and chemical limitations which limit their usage in the chemical process industry (CPI). This project focuses on developing high temperature, chemically stable, high gas flux perfluoromembranes for usage in harsh (chemical, temperature) environments consistent with existing needs in the CPI. In Phase I, high flux thin perfluoropolymer films will be fabricated on chemically resistant porous supports. Single gas testing of perfluoromembranes will initially be done using harsh gases and appropriate secondary gases. This will be followed by single gas evaluation of thin film perfluoromembranes and then by evaluation of mixed gases on these membranes. Focus during Phase I will be on chlorine and hydrogen separations associated with the chloralkali industry with care taken to avoid explosive conditions. Engineering simulations will be done in parallel to actual tests. In Phase II these units will be scaled up for pilot testing in the chloralkali industry and other harsh chemical separations.

\section{Consultec Scientific, Inc. Knoxville, TN 37932}

\section{A Miniature Multi-Chemical Sensor Using Micro-Cantilevers \\ Hunter, S.R. 423-675-4333

This project will result in the development of a revolutionary class of miniature chemical sensors that are as much as 1000 times more sensitive, up to 1000 times smaller, and considerably less expensive than currently available multichemical sensors. This will be achieved by utilizing microcantilever technology, such as that commonly used in scanning force microscopy. Both the resonance frequency and the bending of these microcantilevers have been shown to vary reproducibly and sensitively (with picogram to sub-picogram sensitivity) as a consequence of adsorption of molecules on their surfaces. In preliminary studies, it has been shown that these microcantilevers can be used to detect a variety of chemicals, ranging from aromatic hydrocarbons and alkyl mercaptans to mercury vapor. Analyte-selective coating of the surface of individual microcantilevers provides specificity for target species. This basic concept will be extended during Phase I to demonstrate detection and identification of key components in a mixture, using multiple coated microcantilevers and pattern recognition analysis. Because numerous microcantilevers may be packaged into an extremely small volume, localized sensing and nearly instantaneous response are facilitated with this approach. Also, the monolithic nature of potential multichemical sensors should minimize sensitivity to extreme temperature or pressure changes by assuring that all active and reference sensor elements are in equilibrium with the same monitored environment. Phase I efforts will be limited to a proof-of-principle demonstration for a limited range of analytes. Once demonstrated, however, extension to numerous other analyte and sample matrix systems can be effected by a change in coating set. Hence, the planned work will provide a highly significant advance in sensor technology with possibilities of developing new sensors for other chemicals

\section{EIC Laboratories, Inc. Norwood, MA 02062}

\section{High Capacity Carbon Anodes for Lithium Ion Batteries \\ Abraham, K.M. \\ 617-769-9450 \\ $\$ 74,500$}

The proliferation in recent years of electronic and portable devices of ever-decreasing sizes and weights has spurred the demand for high energy density rechargeable batteries. Lithium ( $\mathrm{Li}$ ) ion batteries can play a major role in fulfilling this need. The unavailability of high capacity carbon anodes, however, has limited the energy density of these batteries. Research to develop a novel high capacity carbon anode suitable for rechargeable $\mathrm{Li}$ ion batteries is planned. Phase I is aimed at gaining a fundamental understanding of the structure-property relations of the carbon as well as identification of experimental conditions leading to a material with the best overall performance of capacity, rate, and rechargeability. The Phase I tasks will include: (1) preparation of carbonaceous materials, (2) characterization of the carbons, and (3) electrochemical evaluation of the carbons in $\mathrm{Li}$ cells.

\section{Materials and Electrochemical Research Corp. Tucson, AZ 85706}

\section{Electrode Materials for Rechargeable Lithium Batteries \\ Loutfy, R.O.} 602-574-1980

Fullerenes are a newly discovered form of carbon (graphite and diamond are the other two forms) with distinctive properties and an unusual molecular structure. Preliminary investigations indicated that fullerene electrode materials exhibit unique electrochemical properties. In this Phase I effort, fullerene will be investigated as novel electrode materials for lithium ion storage and supply for rechargeable lithium batteries. Fullerene electrodes will be fabricated by various techniques and fully characterized. The nature of the electrochemical charging 
and discharging of fullerene nanotubes as a function of operating conditions will be established. The lithium intercalated fullerene $\left(\mathrm{C}_{\mathrm{n}} \mathrm{Li}_{\mathbf{x}}\right)$ electrodes will also be characterized, including their capacity, stability, charge and discharge characteristics, and efficiency to establish their viability for a high-efficiency secondary battery as a power source for portable electronic equipment. Fullerenes are a newly discovered material with potentially unique properties. Preliminary results indicate that fullerene electrodes could be used to store energy with applications in secondary batteries. The feasibility of this concept could lead to novel electrochemical systems for energy storage and generation.

\section{Membrane Technology \& Research, Inc. Menlo Park, CA 94025}

\section{Highly Selective Membranes for the Separation of Organic. Vapors Using Superglassy Polymers. \\ Pinnau, I. 415-328-2228:}

$\$ 750,000$

The separation of organic vapors from permanent gases is a process of considerable industrial: importance. Applications include the separation of organic vapors from industrial effluent aị streams, of hydrocarbons from refinery hydrogen purge streams, and of higher hydrocarbons from methane in natural gas processing. Membranes that are preferentially permeable to the minor organic component are being developed for these applications. For an efficient process, a high organic vapor/permanent gas. selectivity and a high vapor flux are required. Rubbery polymers, which are organic vapor selective, are in limited: commercial use, but have inadequate selectivity for many applications. In Phase I the use of poly(1-trimethylsilyl-1propyne) (PTMSP), a high-free-volume, superglassy polymer, was investigated as an advanced vapor separation membrane material. PTMSP membranes are extraordinarily effective at separating organic vapors from permanent gases. Membrane selectivities are two to ten times higher. Furthermore, good long-term stability of PTMSP in an organic vapor environment was demonstrated. In Phase II optimized PTMSP composite membranes will be developed and the best membrane formed into industrial-size modules for pilot-testing. The long-term performance and stability of PTMSP composite membranes will be tested in a pilot system with hydrocarbon/hydrocarbon and hydrocarbon/methane mixtures.

\section{Microsensor Systems, Inc. Bowling Green, KY 42103}

\section{Advanced Chemiresistor Devices as Micron Size Sensors for the Rapid, On-Line Measurement of Chemical Vapors Wohltjen, $H$. 502-745-0099 $\$ 73,005$}

An advanced chemical microsensor with potential for highly localized (micron-size scale) sensing is planned as a rapid response, in-situ chemical vapor detector for, chemical process monitoring. Such chemical microsensors will be of considerable value for the real-time monitoring of manufacturing processes and detecting changes in the composition of chemical waste streams. The new sensors are based on the incorporation of small concentrations of conducting particles in polymer matrices to form chemically sensitive semiconductors. When exposed to chemical vapors that are specifically absorbed by the polymer, the matrix will swell and change the particle contact geometries and interparticle distances, thus increasing the electrical resistance. Thin, uniform layers of polymer/ metal mixtures can be formed on very small quartz or alumina substrates etched with a laser beam to form long, narrow serpentine lines with length to width ratios greater than 1000 to 1 , thus giving devices with sensitivities at the parts per billion level. Among the advantages of polymer/metal chemiresistors is that their size can be reduced to micron-size dimensions using standard planar electronic microfabrication technology. They require minimal electronic circuitry for operation and can be readily installed in a chemical vapor stream where they will respond rapidly to small changes in chemical composition and concentration. Also, they will operate at high pressures, are very inexpensive, and consume very little power. The Phase. I study will focus on determining the overall feasibility of the approach. Different polymer/: metal particle combinations will be examined as well as the level of particle loading. Sensitivity response time and selectivity will be examined for the selected polymer/ metali combinations.

\section{TDA Research, Inc. Wheat Ridge, CO 80033}

\section{Catalysts for Heterogeneous. Alkene Hydroformylation. \\ Srinivas, G: 303-940-2321}

$\$ 100,000$

Alkene hydroformylation is the reaction of an alkene with synthesis gas (carbon monoxide/hydrogen) to form aldehydes. Current worldwide production of aldehydes and alcohols from hydroformylation exceeds five million tons/year. The aldehydes are primarily hydrogenated to alcohols which are used to make plasticizers, solvents, diluents, and agricultural products. Both cobalt (Co) and rhodium (Rh) carbonyl complexes are active for hydroformylation; the $\mathrm{Rh}$ complexes are $10^{2}-10^{4}$ times more active than the Co complexes. However, the homogeneous process has the following two major problems: (1) Since the catalyst and the products of the reaction are in the same phase, catalyst recovery needs to be accomplished by energy intensive distillation processes. (2) Excess phosphene ligands and the carbonyl complex catalysts used are extremely toxic, making the process environmentally unfriendly. The problems associated with homogeneous hydroformylation may be solved if the reaction is carri ed out in the vapor phase over a solid catalyst. Prior efforts at heterogenizing the process using inert carriers for the complexes have failed because of poor stability of the catalysts and low aldehyde selectivity. In Phase I a novel class of promoted Rh catalysts will be synthesized. The catalysts will be characterized and their. activity towards alkene hydroformylation and, aldehyde selectivity will be investigated. Finally, an engineering analysis will be performed to determine the economic and environmental benefits derived; from using these novel 
catalysts instead of the conventional homogeneous catalysts in the solvent-based system. 



\section{SPECIAL FACILITIES}

The special facilities described on the following pages are supported wholly or partly by the Division of Chemical Sciences. They represent an assembly of unique and/or expensive equipment which would be costly to develop elsewhere. They represent research resources for the general scientific community, and qualified scientists from laboratories outside the host laboratory are encouraged to make use of them. However, any activity that can be carried out through commercially available laboratories is not appropriate for these DOE-supported facilities.

The process by which an off-site scientist can use a facility is discussed in each facility summary. For the National Synchrotron Light Source, the Stanford Synchrotron Radiation Laboratory, and the Combustion Research Facility, see the section "User Mode." For the remaining facilities, see "Collaborative Use," which describes the different procedures used at the individual facilities.
Each of the facility summaries also gives the names of individuals to contact for further information, a general description of the facility, and a list of technical data on the primary available instrumentation.

The Office of Basic Energy Sciences also supports other facilities not summarized here. Information concerning these can be obtained by contacting the Director of the Division of Materials Sciences, ER-13, U.S. Department of Energy, Germantown, MD 20874.

Budgets for the operation of those facilities specifically funded as Chemical Sciences Facilities (KC-03-01-04) are given below.
Location

\section{Brookhaven National Laboratory}

Oak Ridge National Laboratory

\section{Sandia National Laboratories, California}

Stanford University

\section{Facility}

National Synchrotron Light Source

High Flux Isotope Reactor

Radiochemical Engineering

Development Center

Combustion Research Facility

Stanford Synchrotron

Radiation Laboratory
Operating Funds

$\$ 7,071,000$

$24,248,000$

$7,070,000$

$4,171,000$

$10,731,000$ 


\title{
PREMIUM COAL SAMPLE PROGRAM (KC-03-02-01)
}

\author{
Chemistry Division \\ Argonne National Laboratory \\ Argonne, IL 60439
}

The purpose of the Premium Coal Sample Program is to provide the coal science community with longterm supplies of a number of premium coal samples that can be used as standards in fundamental research. The premium coal samples distributed through this program are as chemically and physically identical, as possible, have well-characterized chemical and physical properties, and are stable over long periods of time. The coals were mined, transported, processed into desired particle and sample sizes, and packaged in environments as free of oxygen as possible. The natural moisture content was also maintainedin order to ensure that the coals are in as pristine and stable a condition as possible.

\section{AVAILABWITY}

Eight coal samples are available to research personnel at a nominal replacement cost A limited quantity. of large pieces, stored under similar inert conditions, is also available on special request.

More than 800 shipments totaling over 22,000 ampoules have been made thus far. A Users Handbook is updated periodically and available upon request (see contact below) or on the World Wide. Web.

\section{PERSON TO, CONTACT FOR INFORMATION}

Dr. Ken B: Anderson

Phone: (708) 252-1928

Chemistry Division, Fax: (7.08) 252-9288

Bldg. 200.

Argonne. National

Laboratory.

9700, South Cass Avenue.

Argonne, IL 60439

E-mail: kbanderson@anl.gov

$\begin{array}{llcc}\text { \#. } & \text { Seam } & \text { State } & \text { C } \\ & \text { Upper Freeport. } & \text { PA } & 85.5 \\ 1 & \text { Wyodak-Anderson: } & \text { WY } & 75.0 \\ 2 & \text { Wyod } & \text { IL } & 77.7 \\ 3 ; & \text { Mlinois \#6 } & \text { PA } & 83.2 \\ 4 & \text { Pittsburgh \#8. } & \text { VA } & 91.1 \\ 5 & \text { Pocahontas \#3 } & \text { UT } & 80.7 \\ 6 . & \text { Blind Canyon. } & \text { WV } & 82.6 \\ 7 . & \text { Lewiston-Stockton } & \text { ND: } & 72.9 \\ 8 & \text { Beulah-Zap } & \text { N. } & \end{array}$

H

$4.7,0$

5.35 .

5.00

5.32

4.44

5.76 :

5.25

4.83 . o.

7.5

18.0 .

13.5

8.8

2.5

11.6.

- $9: 8$

20.3
$\mathbf{S}$

2.32:

$0: 63$;

$4: 83$

$2: 19$.

0.66

$0: 62$

0.71

0.80
Ash

$13: 2$

8.8

15.5

$9: 2$

4.8

4.7 .

$19: 8$

9.7 


\title{
PULSE RADIOLYSIS FACILITY (KC-03-01-01)
}

\author{
Chemistry Division \\ Argonne National Laboratory \\ Argonne, IL 60439
}

The Argonne electron pulse radiolysis facility has been and is being used for a wide variety of experiments. Designed for chemical research, the moderate energy of the electron accelerator (maximum energy of $21 \mathrm{MeV}$ transient mode, $14 \mathrm{MeV}$ steady state mode) generates transient species without excessive nuclear activation. The pulse width can be varied from $25 \mathrm{ps}$ to $10 \mathrm{~ms}$. In addition, a 5-ps pulse with the same peak current as the 25-ps pulse has been developed. In liquids, transient concentrations up to $20 \mathrm{mM}$ can be generated with the 25-ps pulse and concentrations more than $10 \mathrm{mM}$ can be generated with the longest pulse. Instrumentation for the measurement of chemical processes allows kinetic spectrophotometric absorption and emission and fast conductivity measurements. A 2-ps streak camera with custom software is available for fast emission measurements. Very high time resolution measurements that make use of the short pulse capability of the LINAC can also be made. All data acquisition equipment is computer interfaced to provide accurate data reduction. Sample preparation and handling facilities are available for solid, and handling facilities are available for solid, liquid, and gaseous samples. Recent nonchemical experiments include the verification of radiation monitors under pulse conditions, the determination of cavity modes induced in cavities by a short pulse of electrons, the verification of the theory of wakefield acceleration, and the effect of high-energy electrons on material properties.

\section{COLLABORATIVE USE}

Collaborative experiments can be arranged with appropriate staff scientists.

\section{PERSON TO CONTACT FOR INFORMATION}

Charles D. Jonah

Chemistry Division

Argonne National

Laboratory

9700 South Cass Avenue

Argonne, IL 60439

Computer address: Jonah@Anlchm.bitnet

Alternative: Jonah@ANLCHM.CHM.ANL.GOV

\section{TECHNICAL DATA}

\section{Energy \\ Transient mode \\ Steady-state mode \\ Average current \\ Pulse repetition rate}

Current/pulse

Transient mode $20 \mathrm{~A}$ peak

Steady-state mode $1.5 \mathrm{~A}$ peak

Picosecond pulse $.25 \mathrm{nC}$ (charge per pulse)

Picosecond (5 ps)

Pulse width

\section{$21 \mathrm{MeV}$ \\ $14 \mathrm{MeV}$ \\ $200 \mu \mathrm{A}$ (maximum) \\ Single pulse to $800 \mathrm{pps}$ \\ (800 pps not possible \\ for all pulses)}

Phone: (708) 252-3471

(708) $252-4993$

\section{$6 \mathrm{nC}$}

$5 \mathrm{ps}$

$25 \mathrm{ps} \quad$ transient mode

4 to $100 \mathrm{~ns}$

0.5 to $10 \mu$ s steady state mode 


\title{
NATIONAL SYNCHROTRON LIGHT SOURCE
}

\author{
Brookhaven National Laboratory \\ Building 725B, P. O. Box 5000 \\ Upton, NY 11973-5000
}

The National Synchrotron Light Source (NSLS) is the largest facility in the U.S. dedicated to the production of synchrotron radiation. Funded by the Department of Energy as a user facility, construction on the NSLS began in 1977 with VUV Ring operation commencing in 1982 and X-Ray Ring operation in 1984. Since then, the facility has undergone a major 4-year upgrade and is continually improved to take advantage of the latest technology in storage rings, beamline optics, and insertion devices.

The NSLS operates two electron storage rings producing high brightness synchrotron radiation in the infrared, visible, ultraviolet, and x-ray regions of the electromagnetic spectrum. Insertion devices installed in the straight sections of the rings provide radiation that is anywhere from one to several orders of magnitude brighter than the radiation from bending magnets. The VUV Ring operates at $800 \mathrm{MeV}$ with a critical energy of $486 \mathrm{eV}$. It has 17 beam ports split into 25 experimental stations, or beamlines, and also supports two insertion devices. The X-Ray Ring operates at $2.5 \mathrm{GeV}, 300 \mathrm{~mA}$, with a critical energy of about $5 \mathrm{keV}$. It has a total of 30 beam ports split into 60 beamlines and currently supports 5 insertion devices: two undulators, a superconducting wiggler, and two hybrid wigglers. There are also a number of beamlines devoted to machine diagnostics and R\&D. The NSLS facility has user laboratories and a wide range of research equipment for basic and applied studies in condensed matter, surface science, photochemistry and photophysics, lithography, crystallography, smallangle scattering, metallurgy, x-ray microscopy, topography, etc. Detailed information about beamline research programs, experimental apparatus, and optical configurations is available from the NSLS User Administration Office.

\section{USER MODES}

Approximately 2300 scientists from more than 350 institutions came to the NSLS to perform research during 1994. The NSLS is a national user facility

available without charge to university, industrial, national laboratory, and government users. In addition, a program is available to assist faculty/student research groups who have limited grant support and wish to defray travel expenses to the NSLS. Proprietary work can be done on a full cost recovery basis with the option to retain title to inventions resulting from research at the NSLS.

There are several ways of using NSLS experimental facilities. A large fraction of the beamlines have been designed and constructed by Participating Research Teams (PRTs). PRTs are comprised of one or more research teams from industry, universities, and other laboratories with large, long-range programs which have been approved by the NSLS Scientific Advisory Committee (SAC). The PRT members are given priority for up to $75 \%$ of their beamline's operational time, and their programs are reviewed by the SAC every three years. Peer-reviewed General User proposals are scheduled on both PRT beamlines and on beamlines built by the NSLS for the general community. The NSLS facility operates throughout the year with beam time scheduled in 4-month cycles. Deadlines for General User proposals are September 30, January 31, and May 31. Information about submitting research proposals, becoming a PRT, or applying for financial assistance may be obtained from the NSLS User Administration Office.

\section{PERSON TO CONTACT FOR INFORMATION}

Eva Z. Rothman,

User Administrator

NSLS Bldg. 725B

Brookhaven National

Laboratory

P. O. Box 5000

Upton, NY 11973-5000

E-mail: ezr@bnl.gov

See also World Wide Web at:

URL = http://www.nsls.bnl.gov/
Phone: (516) 282-7114

Fax: (516) 282-7206 


\section{NSLS TECHNICAL DATA*}

\section{Storage Rings}

VUV

X-Ray

\section{Research area}

Absorption Spectroscopy

Circular Dichroism

High Pressure Physics

\section{High Q-Resolution Scattering \\ Imaging: Medical \\ Tomography \\ X-Ray Microprobe \\ X-Ray Microscopy/Holography \\ X-Ray Topography \\ Infrared Spectroscopy \\ Lithography \\ Nuclear Physics \\ Photoemission Spectroscopy \\ Photoionization \\ Protein Crystallography \\ Radiometry \\ Small Angle Scattering: \\ Biology \\ Materials Science}

Small Molecule Crystallography:

Powder

Single Crystal

Standing Waves

Surface Scattering/

X-Ray Reflectivity

Time Resolved Fluorescence

UV Reflectometry

X-Ray Emission Spectroscopy

\section{Key features}

17 ports; $\mathrm{E}_{\mathrm{c}}=25.3 \AA ; 0.745 \mathrm{GeV}$ electron energy

30 ports; $\mathrm{E}_{c}=2.48 \AA \AA 2.584 \mathrm{GeV}$ electron energy

\section{Wavelength range (Å)}

0.35 to 2480
10.3 to 5904
1 to $10,000 \mu \mathrm{m}$
WB; 0.12 to 1.24
WB; 0.12 to 6.20
WB; 0.12 to 1.24
WB; 0.12 to 3.10
WB; 0.12 to 3.10
10 to 80
WB; 0.41 to 3.10
1 to $10,000 \mu \mathrm{m}$
124 to 4133
-
2.10 to 6200
2.10 to 4133
WB; 0.41 to 3.10
WB; 8.27 to 248
0.66 to 5.90
0.36 to 6.20

WB; 0.12 to 3.10

0.21 to 6.20

WB; 0.62 to 6.89

WB; 0.48 to 6.20

1393 to 5904

WB; 8.27 to 6200

2.48 to 50

\section{Energy range $(\mathrm{eV})$}

5 to 35,000

2.1 to 1200

0.124 to $1240 \mathrm{MeV}$

WB; 10,000 to 100,000

$\mathrm{WB} ; 2000$ to 100,000

WB; 10,000 to 100,000

WB; 4000 to 100,000

WB; 4000 to 100,000

155 to 1240

WB; 4,000 to 30,000

0.124 to $1240 \mathrm{MeV}$

3 to 100

80 to $400 \mathrm{MeV}$

2 to 5900

3 to 5900

WB; 4000 to 30,000

WB; 50 to 1500

2100 to 18,800

2000 to 34,000

WB; 4000 to 100,000

2000 to 59,400

WB; 1800 to 20,000

WB; 2000 to 26,000

2.1 to 8.9

WB; 2 to 1500

248 to 5000

\section{Number of beamlines}

24
2
2
2
15

2

3

3

1

2

2

1

1

19

3

6

1

2

4

4

7

2

10

1

2

2

*From 1993 NSLS User's Manual-BNL 48724.

$+\mathrm{WB}=$ White Beam 
JAMES R. MACDONALD LABORATORY (KC-03-01-03)

\author{
Department of Physics \\ Kansas State University \\ Manhattan, KS 66506
}

The laboratory operates a 7-MV tandem accelerator, a 9-MV superconducting linear accelerator (LINAC) and a cryogenic electron beam ion source (CRYEBIS) for the study of ion-atom collisions with highly charged ions. The tandem can operate as a standalone accelerator with six dedicated beam lines. The LINAC is operated as a booster accelerator to the tandem. The tandem-LINAC combination has four beam lines available. The CRYEBIS is a stand-alone facility for studying collisions with bare ions at low velocity. An ion-ion collision facility using the CYREBIS and a new ECR ion source are under development. The laboratory has a variety of experimental apparatus for atomic physics research. These include recoil ion sources, Auger electron spectrometers, $\mathrm{X}$-ray spectrometers, and a 45-inch-diameter scattering chamber. The laboratory is available to users who require the unique facilities of the laboratory for atomic collision experiments.

\title{
TECHNICAL DATA
}

\section{EN Tandem}

\section{Beams \\ Terminal voltages \\ Output currents}

Repetition rate

Magnet limitations

\author{
Most elements \\ 0.3 to $7 \mathrm{MV}$ \\ Up to $10 \mu \mathrm{A}$ \\ depending on the \\ ion species and \\ the charge state \\ DC or 3-ns pulses at \\ rates up to $4 \mathrm{MHz}$, \\ or $12 \mathrm{MHz}$ operation \\ $\mathrm{ME} / \mathrm{q}^{2} \leq 150$
}

\section{COLLABORATIVE USE}

Users are encouraged to seek a collaborator on the staff or to submit a brief proposal to the Laboratory Director.

\section{PERSON TO CONTACT FOR INFORIMATION}

Patrick Richard, Director Phone: (913) 532-6783 James R. Macdonald

Laboratory

Department of Physics

Kansas State University

Manhattan, KS 66506-2604

E-mail: Richard@phys.ksu.edu
LINAC Booster

\begin{tabular}{|c|c|}
\hline Acceleration field & $9 \mathrm{MV}$ \\
\hline Resonators & $\begin{array}{l}\text { Split-ring, super- } \\
\text { conducting } \mathrm{Nb}, \\
\text { operated at } 97 \mathrm{MHz}\end{array}$ \\
\hline Beam repetition rate & $\begin{array}{l}12 \mathrm{MHz} \text { with } 75 \% \\
\text { of beam bunched }\end{array}$ \\
\hline Mass limitation & $\begin{array}{l}M<100 \mu \text { due to } \\
\text { injection energy }\end{array}$ \\
\hline
\end{tabular}

CRYEBIS

Beams

Beams energy

Output currents
Up to bare ions of $\mathrm{C}, \mathrm{N}, \mathrm{O}, \mathrm{F}, \mathrm{Ne}, \mathrm{Al}$, and Ar. Up to $\mathrm{Kr}^{36+}$ and $\mathrm{Xe}^{47+}, \mathrm{Fe}$ beams under development A few to $200 \mathrm{keV} / \mathrm{q}$ $10^{5}$ to $10^{8} \mathrm{parts} / \mathrm{s}$ 


\author{
Notre Dame Radiation Laboratory \\ University of Notre Dame \\ Notre Dame, IN 46556-0579
}

The Notre Dame pulse radiolysis facility is based on an $8-\mathrm{MeV}$ linear accelerator which produces pulses of 2 to 100 nanosecond duration. It is fully instrumented for computerized acquisition of optical and conductivity information on radiation chemical intermediates having lifetimes of $5 \mathrm{~ns}$ and longer. An excimer laser/dye laser combination is available for use at the pulse radiolysis facility for double-pulse experiments involving photolysis of radiolytic transients, Energies of $\sim 400 \mathrm{~mJ}$ at $308 \mathrm{~nm}$ and $\sim 50 \mathrm{~mJ}$ at various near-UV and visible wavelengths are available, For typical absorption studies, where one produces $10^{-5} \mathrm{M}$ of intermediates, spectral and kinetic information can be obtained on species having extinction coefficients in excess of $100 \mathrm{M}^{-1} \mathrm{~cm}^{-1}$ : Conductometric methods in aqueous solution cover the $\mathrm{pH}$ range of 3 to 11 . Data are recorded digitally and stored in magnetically readable form for rapid off-line examination of spectral and kinetic details.

\section{COLLABORATIVE UUSTE}

Experiments may be arranged by a proposal to the laboratory director or through collaborations with appropriate staff scientists,

\section{PERSON TO CONTACT FOR INFORMATION}

J. Bentley

Assistant Director

Phone: (219) 631-6117

Notre Dame Radiation

Laboratory

Notre Dame, IN 46556

\section{TECHNICAL DATA}

\begin{tabular}{|c|c|}
\hline Electron source & 8-MeY linear accelerator \\
\hline Operating mode & $\begin{array}{l}\text { Single pulse, with } \\
\text { signal averaging }\end{array}$ \\
\hline Data collection & Workstation (DOS/Intel 486) \\
\hline Pulse width & $2-100 \mathrm{~ns}$ \\
\hline Time resolution (RC) & $2 \mathrm{~ns}$ \\
\hline Pulse current & $\mathrm{Up}$ to $4 \mathrm{~A}$ \\
\hline Repetition frequency & $0.2 \mathrm{~s}^{-1}=$ \\
\hline \multicolumn{2}{|c|}{ Optical absorption measurements } \\
\hline Spectral region & 210 to $750 \mathrm{~nm}$ \\
\hline Sensitivity & \pm 0.00002 absorbance \\
\hline \multicolumn{2}{|l|}{ Conductivity } \\
\hline pH range & 3 to 11 \\
\hline Șensitivity & $\pm 5 \mathrm{mhos} / \mathrm{cm}$ \\
\hline
\end{tabular}

\title{
HIGH FLUX ISOTOPE REACTOR (KC-03-01-04)
}

\author{
Research Reactors Division \\ Oak Ridge National Laboratory \\ Oak Ridge, TN 37831
}

Since it began full-power operations in 1966, the High Flux Isotope Reactor (HFIR) at Oak Ridge National Laboratory (ORNL) has been one of the world's most powerful research reactors. A primary purpose of the HFIR is the production of ${ }^{252} \mathrm{Cf}$ and other transuranium isotopes for research, industrial, and medical applications. These materials are produced in the flux trap in the center of the HFIR fuel element where a working thermal-neutron flux of $2.5 \times 10^{15}$ neutrons $/ \mathrm{cm}^{2} \mathrm{~s}$ ) is available to irradiate the target material. Additional irradiation facilities are also provided in the beryllium reflector.
Beyond its contributions to isotope production, the HFIR also provides for a variety of irradiation tests and experiments that benefit from the exceptionally high neutron flux available. In the fuel element flux trap, a hydraulic rabbit tube provides access to the high thermal-neutron flux in the reactor for shortterm irradiations, and other positions are ideal for fast-neutron irradiation-damage studies. A modification of the flux trap experiment facilities in 1986 has provided two locations in the maximum flux region that can accommodate instrumental capsules and engineering loops. The beryllium reflector contains 
numerous experimental facilities with thermalneutron fluxes up to $1.0 \times 10^{15}$ neutrons $/\left(\mathrm{cm}^{2} \cdot \mathrm{s}\right)$. These facilities can accommodate static experimental capsules, complex fuel-testing engineering loops, and special experimental isotope irradiations, the targets for which are prepared by ORNL or other qualified users.

Another major use of the HFIR is for neutronscattering experiments to reveal the structure and dynamics of a very wide range of materials. The reactor has four horizontal beam tubes with inner diameters of $10 \mathrm{~cm}$ that extend outward from the reactor core at the midplane of the reactor. Beam tube HB-2 extends radially from the reactor centerline, and beam tube HB-3, which extends tangentially from the core, is offset $34 \mathrm{~cm}$ from the reactor center. A third tube is aligned on a tangential line $39 \mathrm{~cm}$ from the reactor centerline with both ends extending outward from the reactor to allow for the installation of two individual facilities. The two ends of this tube are designed HB-1 and HB-4. A scattering block of beryllium has been placed at the centerline between beam tubes HB- 1 and HB-4, effectively making them into two tangential tubes.

The neutron-scattering instruments installed on the horizontal beam tubes are used in fundamental studies of materials of interest to solid-state physicists, chemists, biologists, polymer scientists, metallurgists, and colloid scientists. These instruments are open to use by university and industrial researchers on the basis of scientific merit, and about 150 to 200 researchers use the experiment facilities at the HFIR each year.

\title{
PERSON TO CONTACT FOR INFORMATION
}

J. E. Lee

Research Reactors

Division

Oak Ridge National

Laboratory

P. O. Box 2008

Oak Ridge, TN 37831

E-mail: leeje@ornl.gov
Phone: (423) 574-8288

Fax:

(423) $547-9175$

\section{RADIOCHEMICAL ENGINEERING DEVELOPMENT CENTER (KC-03-01-04)}

\author{
Chemical Tèchnology Division \\ Oak Ridge National Laboratory \\ Oak Ridge, TN 37831
}

The objective of the base program at the Radiochemical Engineering Development Center (REDC) is to supply transplutonium elements for use in research. The REDC is the distribution center for the DOE/ER heavy-element research program. Target rods are fabricated at REDC, irradiated in the High Flux Isotope Reactor (HFIR), and processed at REDC for separation, recovery, and purification of the heavy actinides up through ${ }^{257} \mathrm{Fm}$. Since their construction in the mid-1960s, REDC and HFIR have provided the western world's supply of elements beyond curium (atomic number 96), either directly or by furnishing starting materials for further nuclear-synthesis reactions. The transuranium element isotopes produced in the REDC are used nationally and internationally to study the basic physics and chemistry of the transuranium elements. They are also being used in research and development, programs relating to environmental effects, biological effects, and waste isolation.
Similar radiochemical separations projects can be and are often carried out in the REDC for other DOE programs. Currently, transplutonium elements are being recovered from targets irradiated at Savannah River for Defense Programs. Also, ${ }^{252} \mathrm{Cf}$ portable neutron sources are prepared for a variety of radiography, activation analysis, and cancer treatment applications. REDC facility management is under the direction of the Assistant Secretary for Nuclear Energy. Base funding is provided by the Office of Energy Research and is supplemented by other agencies when their projects are carried out.

\section{PERSON TO CONTACT FOR INFORMATION}

R. M. Wham

Phone: (423) 576-7783

Chemical Technology Division

Oak Ridge National Laboratory

P. O. Box 2008

Oak Ridge, TN 37831-6384 


\author{
Sandia National Laboratories, California \\ Livermore, CA 94551-0969
}

Current activities at the Combustion Research Facility (CRF) supported by the Division of Chemical Sciences emphasize the development and the applications of new diagnostic techniques to the study of basic flame processes, research in fundamental chemistry in combustion, as well as analytical studies of reacting turbulent flows. (These programs are individually described elsewhere in this publication.) The active program of visitors to the facility, including senior researchers, graduate students, and postdoctoral researchers supported through the Chemical Sciences Division, is described below.

Facility support, through the Chemical Sciences Division, includes operation and continued development of the CRF central lasers. Several are available. The tunable dye laser (Diana) is used by Sandia staff and visiting scientists for single-shot temperature, density, and species concentration measurements, and for two- and three-dimensional imaging of turbulent flames. A multipurpose laser system (Sirius) consists of a frequency-doubled Nd:YAG laser and a pulseamplified ring dye laser. When the ND:YAG laser is operated in single-axial mode in combination with the ring dye laser, the spectral resolution for CARS and other nonlinear spectroscopy experiments (performed in any of the CRF laboratories via the beam distribution system) is as small as a few thousandths of a wave number. Sirius is used also for CARS measurements in flames with large luminous backgrounds (e.g., heavily sooting flames laden with coal particles). A third central laser (Dyeblaster) consists of a frequency-doubled $\mathrm{Nd}$ :YAG laser and is used routinely to pump dye lasers in user laboratories throughout the CRF.

In addition, DOE/Energy Efficiency and Renewable Energy sponsors programs at the CRF in combustion technologies and materials processing by design, DOE/Fossil supports programs in coal combustion and related diagnostics development, DOE/BES Engineering Science supports advanced analysis of turbulent flows and DOE/BES Materials Sciences support programs in combustion-related materials research.
Complete facilities for resident and visiting researchers are available: offices for 60 staff, a meeting room accommodating 250 people, a laboratory building housing 24 independent experiments, special facility laser systems, a network of computer workstations, and access to supercomputers.

In specific instances, proprietary research can be carried out at the CRF. For this type of work, the DOE will be reimbursed on a full cost recovery basis for the use of all CRF resources. Details of a DOE Class Waiver for patent rights are available.

\section{USER MODE}

Qualified scientists are encouraged to take advantage of the specialized resources available at the CRF. Prospective participants should submit a brief proposal to the laboratory director. Criteria for selection include technical merit, the extent to which CRF facilities are used, overlap with $\mathrm{DOE}$ program objectives, and the availability of specific equipment.

In general, the CRF will host visiting scientists to use the special-purpose lasers, work with resident staff, make use of computers and codes, and set up experiments. Visitors pay for their own salary, travel, housing, meals, and other local expenses. Facility lasers, apparatus, technicians, instrumentation, computers, and support-group services are provided without charge for research that is not proprietary. Research results from nonproprietary projects are expected to be published and disseminated.

For scientists with active government contracts, support for CRF research often can be arranged on an informal basis with the contract manager. There are opportunities for faculty, postdoctoral scientists, and graduate students to obtain Sandia support for combustion-related research at CRF. 
W̛illiam J McLiean, Director

Combustion and Materials Ściènce ànd Tèchnology

Center

Sandia National

Làboratories

Livermor̈e, CA 94551-0969

or

George A. Fisłk, Manger

Combustion Sciences

Progràims

Sandia Nationàl

Laboratories

Livermore, CA $94551-0969$
Phone: (510) $2944=2687$

\section{TECHNICAL DATA}

\section{Eqqúipment \\ Flashlamp-pumped, tunable dye laser}

\section{Key features}

Long pùlse, high energy, high averäge power: 2-jis pulse length 5 J/Pulse, $5 \mathrm{~Hz}$

Tunable 440 to $620 \mathrm{~nm}$ 0.3-nm bandwidth

Multipurpose pulsed laser system

Low-pressure flames

Atmospheric flames
Vertical tưrbủlënt
diffusion flame

High peak powier, high resolution double YAG and tunable dye lasers: Single mode capability 10 to $500 \mathrm{~mJ} / \mathrm{pulse}$ 10 to $29 \cdot \mathrm{ns} / \mathrm{pulse}$

\section{0 torr to 1 atm Mass spectrometer sampling probe LIF 'detection' of radicals}

\section{Diffusion and premixed flames}

Öpen-circuit, induceddraft wind tuinnel "with co-flowing axisymmetric fuel jet: 30 -by 180 -čm viëwing section to $6000 \mathrm{scfm}$ flow

\section{Èquipmènt \\ Combustion bomb \\ In'ternal combustion research devices}

Experimental
diagnostics research

Tưribulent flame structure laboratory

Burner Engineering Firing rates from $100 \mathrm{~m}$ to Research Laboratory ' $3 \mathrm{~mm}$ Btu/hr capabilities for air/fuel preheat, fuel gas recirculation, and hưmidification. Continuous monitoring of flue'gas $\mathrm{O}_{2}, \mathrm{CH}_{4}, \mathrm{CO}_{2}$, $\mathrm{CO}, \mathrm{NO}$, and $\mathrm{NO}_{2}$ optical diagnostics for particle and species concentrations and temperature.

Highly repeatable environment for homogenous charge, diesel combustion, and pulse

Nonlinear optical Rayleigh, Mie, and Raman 2-D imaging temperature. 


\title{
STANFORD SYNCHROTRON RADIATION LABORATORY (KC-03-01-04)
}

\author{
Stanford Synchrotron Radiation Laboratory \\ MS 69, P. O. Box 4349 . \\ Stanford, CA 94309-0210
}

The Stanford Synchrotron Radiation laboratory (SSRL) is a national user facility which provides synchrotron radiation, a name given to $x$-rays or light produced by electrons circulating in a storage ring at nearly the speed of light. These extremely bright $\mathrm{x}$-rays can be used to investigate objects of atomic and molecular size, allowing a wide variety of research in basic and applied studies on the structure of matter. The facility, which provides 25 experimental stations on 21 beam ports as well as ancillary equipment, is used by researchers from industry, government laboratories and universities in many areas, including the fields of biology, chemistry, environmental molecular science, geology, materials science, electrical engineer, chemical engineering, physics; astronomy, and medicine.

\section{Vacuum Ultraviolet Studies (VUV)}

Research utilizing the VUV and soft $\mathrm{x}$-ray radiation includes (a) the determination of electronic states in metals, semiconductors, magnetic systems, superconductors and other interesting materials; (b) properties of ultra-thin layers and small clusters; (c) kinetic processes in a variety of materials; and (d) lithography and dynamic process of chemisorbed gases.

\section{Structural Molecular Biology}

$\mathrm{X}$-rays are used for research in structural molecular biology, including (a) protein structures and functions through diffraction studies in the crystalline state; (b) protein structures through extended x-ray absorption fine structures studies; (c) dynamic fluctuations in biological systems; (d) the nature of membrane and membrane protein interactions; and (e) the structure and function of metal sites in metalloproteins and metalloenzymes. Specialized facilities for protein crystallography are available. A new high brightness wiggler beam line with 3 experimental stations for structural molecular biology is under construction and will be commissioned in 1996.

\section{X-Ray Studies of Condensed Matter}

Research utilizing $\mathrm{x}$-rays for studies of condensed matter include the following areas: (a) structures of amorphous materials, catalysts and environmentally interesting systems; (b) structures of and phase transitions in surfaces and thin surface layers; (c) kinetics of structural changes in materials; (d) chemical reactivities in the gas phase; (e) nuclear resonant scattering; and ( $f$ ) fundamental $x$-ray scattering and absorption physics. A new beam line for environmentally relevant studies is in the initial phases of construction.

\section{Synchrotron Radiation Sources}

Considerable research is also underway in the development of accelerators and devices inserted into the accelerators to produce more intense or brighter synchrotron radiation.

\section{USER MODE}

SSRL is currently used by approximately 950 scientists from over 100 US and foreign based institutions. Scientists gain access to the facility through a refereed:proposal system. Proposals are due May 1 and October 1 each year. The booklet "User Guide" available from SSRL contains information pertinent to proposal submittal: To date, 2336 proposals for research have been received.

\section{PERSON TO CONTACT FOR INFORMATION}

Katherine Cantwell: Phone: (415) 926-3191 Manager; User Research Administration.

SSRL

MS 69, PO Box 4349

Stanford; CA 94309-0210 


\begin{tabular}{|c|c|c|c|c|c|c|}
\hline $\begin{array}{l}\text { Horizontal } \\
\text { angular } \\
\text { acceptance } \\
\text { (Mrad) }\end{array}$ & $\begin{array}{l}\text { Mirror } \\
\text { cutoff. } \\
\text { (keV) }\end{array}$ & Monochromator & $\begin{array}{c}\text { Energy } \\
\text { range } \\
(\mathrm{eV})\end{array}$ & $\begin{array}{c}\text { Resolution } \\
\Delta \mathbf{E} / \mathbf{E}\end{array}$ & $\begin{array}{l}\text { Approximate } \\
\text { spot size, } \\
\text { hgtxwdth, mm }\end{array}$ & $\begin{array}{c}\text { Dedicated } \\
\text { instrumentation }\end{array}$ \\
\hline
\end{tabular}

Wiggler Lines-X-ray
End stations
$4-2$ (4 periods)
Focused
Unfocused
6-2 (27 periods)
Focused
Unfocused
7-2 (4 periods)
Focused
Unfocused
10-2 (15 periods)
Focused
Unfocused
Side Stations
$4-1$
$4-3$
Focused
Unfocused
$7-1$
$7-3$
VUV/soft X-ray stations
$5-3$ multi-undulator
$5-2$ multi-undulator
$10-1$

X-ray

$\begin{array}{ll}1-4 & 2 \\ 1-5 & 1 \\ 2-1 \text { (focused) } & 4 \\ 2-2 & 1 \\ 2-3 & 1 \\ \text { VUV/soft X-ray } & \\ 3-1 & \\ 3-3 & 2 \\ 3-4 & 8-10 \\ 8-1 & \\ 8-2 & 0.6 \\ 1-2 & \end{array}$

Variable

Double crystal

Double crystal

Double crystal

Curved crystal

Double crystal

4 Gratings

6m SGM

Curved crystal

Double crystal

Double crystal

None

Double crystal

2.0
$8-10$

0.6

12
5.0

4.0
Insertion Devices Stations

2,400 to 10,200
2,400 to 45,000
2,050 to 21,000
2,050 to 32,000
2,400 to 10,200
2,400 to 45,000
2,400 to 21,000
2,400 to 45,000
2,400 to 45,000
2,400 to 20,000
2,400 to 45,000
6,000 to 13,000
2,400 to 45,000

$\begin{array}{ll}\sim 5 \times 10^{-4} & 1.0 \times 3.0 \\ -10^{-4} & 2.0 \times 20.0 \\ \sim 5 \times 10^{-4} & 1.0 \times 4.0 \\ \sim 10^{-4} & 2.0 \times 20 .\end{array}$

$\sim 10^{-4}$

$\sim 5 \times 10^{-4}$

$\sim 10^{-4}$

Six-circle diffractometer

$2.0 \times 20.0$

$\sim 5 \times 10^{-4}$

$\sim 10^{-4}$

$\sim 10^{-4}$

$\sim 8 \times 10^{-4}$

$\sim 10^{-4}$

$2.0 \times 20.0$

$0.15 \times 20$

$2.0 \times 20.0$

2,400 to 45,000

10 to 450
10 to 1,200

$0.5^{-1} \times 10^{-3}$

250 to 1,200

$22 \times 10^{-4}$

$1 \mathrm{~mm}^{2}$

$\leq 1 \mathrm{~mm}^{2}$

Bending Magnet Stations

\begin{tabular}{|c|c|c|c|c|}
\hline $\begin{array}{l}\text { Curved crystal } \\
\text { Double crystal } \\
\text { Double crystal } \\
\text { None } \\
\text { Double crystal }\end{array}$ & $\begin{array}{l}6,700 \text { to } 10,800 \\
2,400 \text { to } 30,000 \\
2,400 \text { to } 8,900 \\
3,200 \text { to } 40,000 \\
2,400 \text { to } 30,000\end{array}$ & $\begin{array}{l}4.0 \times 10^{-3} \\
-10^{-4} \\
\sim 5 \times 10^{-4} \\
\sim 5 \times 10^{-4}\end{array}$ & $\begin{aligned} 0.25 & \times 1.0 \\
2 & \times 17 \\
2 & \times 6 \\
4 & \times 22 \\
2 & \times 20\end{aligned}$ & $\begin{array}{l}\text { Small angle scattering detector } \\
\text { Area detector/CAD }-4\end{array}$ \\
\hline $\begin{array}{l}\text { Grasshopper } \\
\text { UHV double } \\
\text { Crystal (jumbo) }\end{array}$ & $\begin{array}{r}25 \text { to } 1,000 \\
800 \text { to } 4,500\end{array}$ & $\begin{array}{l}\Delta \lambda=0.05 \text { to } 2 \AA \\
\sim 5 \times 10^{-4}\end{array}$ & $\begin{array}{l}1.0 \times 1.0 \\
1.5 \times 1.5\end{array}$ & \\
\hline Multilayer & 0 to 3,000 & $\begin{array}{l}\text { White or } \\
\Delta \lambda \lambda=0.6 \%\end{array}$ & $2 \times 8$ & $\begin{array}{l}\text { Vacuum diffractometer } \\
\text { lithography exposure station }\end{array}$ \\
\hline $\begin{array}{l}\text { 6m TGM } \\
6 \mathrm{~m} \text { SGM } \\
6 \mathrm{~m} \text { TGM }\end{array}$ & $\begin{array}{c}8 \text { to } 180 \\
150 \text { to } 1,000 \\
8 \text { to } 90\end{array}$ & $\begin{array}{l}-1 \times 10^{-3} \\
-1 \times 10^{-4} \\
-1 \times 10^{-3}\end{array}$ & $\begin{array}{l}\leq 1 \mathrm{~mm}^{2} \\
\leq 1 \mathrm{~mm}^{2} \\
1.0 \times 1.0\end{array}$ & \\
\hline
\end{tabular}




\section{FY 1995 EQUIPMENT FUNDS}

Outlay funds of the Office of Basic Energy Sciences are divided among operating, equipment, and construction funds. Equipment funds are provided primarily to DOE laboratories. (Operating funds provided to other projects are used for all costs, including purchase of necessary equipment.) FY 1995 equipment funds for Chemical Sciences programs were assigned as follows:

\section{Facility}

Ames Laboratory

Argonne National Laboratory

Brookhaven National Laboratory

Idaho National Engineering Laboratory

E. O. Lawrence Berkeley National Laboratory

Lawrence Livermore National Laboratory

Los Alamos National Laboratory

National Renewable Energy Laboratory

Notre Dame Radiation Laboratory

Oak Ridge National Laboratory

Pacific Northwest Laboratories

Sandia National Laboratories California

Sandia National Laboratories New Mexico

Stanford Synchrotron Radiation Laboratory

\section{Equipment}

Funds

$\$ 431,000^{*}$

$2,167,000 *$

$2,256,000$

37,000

$1,284,000$

28,000

102,000

216,000

379,000

$1,497,000$

767,000

672,000

$1,345,000$

*In addition to the amounts shown, funds for General Purpose Equipment are provided to these laboratories. The amounts are $\$ 150,000$ for Ames Laboratory and $\$ 2,000,000$ for Argonne National Laboratory. 



\section{SELECTED TOPICS OF GENERAL INTEREST}

\section{ACTINIDE CHEMISTRY}

$95,106,107,108,109,110,111,400,402$

\section{ADVANCED BATTERY TECHNOLOGY}

$117,118,119,120,121,122,123,124,125,126,127,422,423$, $424,425,426,427,428,429,430,431 ; 432,438$

\section{ANALYSIS}

General

$87,97,367,381,395,433$

Mass Spectrometry

$86,92,99,100,101,103,370,372,380,382,3833,390$

Optical Spectrometry

$88,90,91,93,98,105,362,365,368,369,371,372,3 \overline{7} 5,37 \overline{7}$; $385,390,394,396,398,399$

\section{ATOMitC PHYSICS}

Collisions

$50,53,54,56,57,59,196,208,246 ; 259,266,268 ; 273 ;, 274$

Fusion

$54,55,56,57,59,196,208,273$

Spectroscopy

$50,51,52,242,248,253,254,258,260 ;, 263 ;, 264,265 ; 27 \dot{1} ; 272$

Theory

$58,243,244,245,247,249,251,252 ; 255 ; 25 \dot{6} ; 25 \overline{7}, 26 \dot{1} ; 262$, $267,269,270$

\section{CATALYSIS}

Chemistry on Model Catalytic Sites

$32,60,180,181,200,217,238,281,320,327,349$

Heterogeneous

$60,62,64,65,71,73,82,84,225,278,280,281,283,284,288$ $289,291,298,299,300,304,306,308,309,313,314,315$, $316,317,318,320,322,326,327,328,332,335,337,338$ $341,342,343,344,345,346,347,350,351,352,354,357$,

Homogeneouis

$60,61,67,68,71,74,75 ; 76,78,84,182 ; 275,277,279 ; 284$ $285,287,290,292,294,295,296,297,302,303,305,307$, $310,311,312,314,316,317,319,325,329,330$; 331, 333 $334,336,339,340,348,353,355,360,361$

Organometallic Chemistry (Car̉bon Moñoxide Related)

$31,67,74,78,182,282,286,291,302 ; 303,310,312,317,325$, $328,330,337,342,348,350,352 ; 356$

\section{COMBUSTION}

\section{Dynamics}

$34,36,39,46,179,183,186,187,189,190,191,192,19 \overline{5} ; 197$ $206,212,214,215,216,219,222,224,226 ; 227,230,232$, $233,235,240$

\section{Kinetics}

$34,35,36,39,42,46,48,178,185,189,198,204,210,218,220$, $223,229,231,236$

Spectroscopy and Analysis

$31,34,36,39,45,46,47,49,183,184,188,189,193,195,202$, $203,205,207,209,210,211,219,224,226,231,236,237,241$

Theory and Modeling

$30,34,36,39,41,42,45 ; 48,178,187,197,199,201,205 ; 206$ $207,210,212,214,216,221,228,229,230$

Turbulence

$45,113,116,403,408,421$

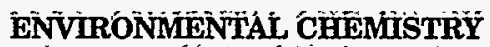

$43,44,60,77,83,193,275 ; 294,317$

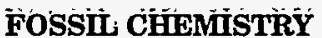

$6 \dot{6} ; 69,7 \overline{7}, 80,84,276,301 ; 321 ; 349$

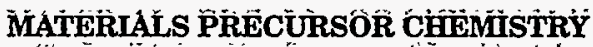

$63,71,72,75,76,279,293,305 ; 323,324$

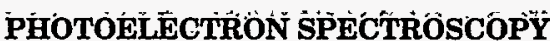

$31,33,37,82,90,188,203 ; 222$

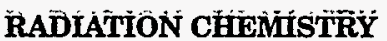

$4 ; 7 ; 11,21 ; 22,24 ; 26 ; 28,29 ; 131 ; 1433,148,149 ; 150,168$

\section{SËṖARATTYÓÕNS}

Chirôñàatographÿ

85 ; 368,$373 ; 378,388 ; 389 ; 397$

Extractióng

89,$66 ; 102 ; 363 ; 364,3 \overline{7} 3,38 \overline{7} ; 391 ; 394 ; 401$

Geñèrail

94; 104, 3779; 385,$392 ; 396$

Meinibrañé

$364 ; 366 ; 374 ; 376,384,386,393 ; 439$

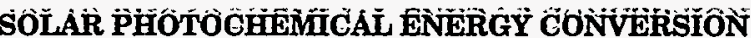

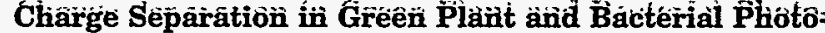
synthesis

1,$2 ; 3,5,6,9,20 ; 79,129 ; 135 ; 141,162 ; 164 ; 175$

Eilectron Trânsfer Ratés añ Mechianisinis

$4,8,11 ; 12,130,131,132 ; 133 ; 135,136,139 ; 140,143,147$, $148,150,151,152,153,154,157,158,159,166 ; 167,170,176$ Microhetérogeneólis Systems

$1 ; \hat{3}_{1} 4,14,16 ; 25,27,130 ; 132,139 ; 145 ; 146 ; 147 ; 152,155$, $157,161,165,167 ; 170 ; 171,174$

Molecuulär M̆òdéĺs

$3,4,8,9,130,132,133 ; 136,143,151 ; 153 ; 157 ; 159 ; 164 ; 170$, $172 ; 176,177$

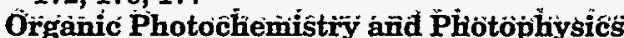

$4,14,24 ; 28 ; 132,133 ; 144 ; 151,165 ; 168,171$

Photoélectrochemistry

4 , 10,$15 ; 16,17 ; 18 ; 19,24 ; 25 ; 134 ; 137 ; 138 ; 140 ; 142,157$ $160,169,170$

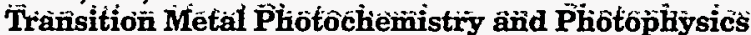

$12,13,23,28,79 ; 128,131,146 ; 150,152 ; 153,156 ; 163,172$ $173,174,176,177$

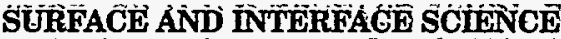

$30,37,38,82,83,90,105,142,194,213,239,280,281,289^{\circ}$, $299 ; 304,308,327,335 ; 342 ; 346,368,388,396 ; 398 ; 418$

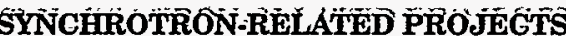

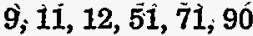

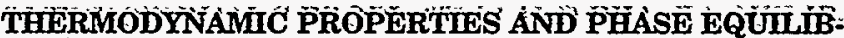
RIIA

$40,70,81,112,114,115 ; 188,203,404,405 ; 406,407,409 ; 410$, $411,412,413,414,415 ; 416 ; 417,419,420$ 



\section{INSTITUTION INDEX}

This index lists the institutions responsible for the projects cited in this publication. The corporate names are entered in standardized forms found in the authority listing International Energy: Research Organizations (ETDE/PUB-3). Each entry includes the project title and citation number.

ANDCARE, Inc.

433

Aerospace Corporation 178

Akron, University of 128,179

Alabama, University of 129

Ames Laboratory, Iowa State University $1,2,3,30,31,60,61,62,63,64,65,66,85,86,87,88,117$

Analysis Consultants 434

Argonne National Laboratory

$4,5,6,7,8,32,33,34,50,51,67,68,69,89,106,118,119$

Arizona State University

$130,180,181,422$

Arizona, University of $182,275,362$

Auburn University 363

Boston College 276

Boston University 131,132

Brandeis University 133

Brigham Young University 364

Brookhaven National Laboratory $9,10,11,12,35,36,37,70,71,90,91,120$

Brown University

365

California Institute of Technology $134,242,277,423$

California State University, Fullerton 243

California, University of, Berkeley 135

California, University of, Davis 278

California, University of, Irvine 279

California, University of, Los Angeles 183

California, University of, Riverside 280

California, University of, San Diego 403

California, University of, Santa Barbara $184,281,282$

Carnegie-Mellon University 283

Catholic University of America 185

Chicago, University of 186,187

Clark Atlanta University 244

Clark University 424

Colorado School of Mines 366,404
Colorado State University $136,137,284,285,367,405$

Colorado, University of

$138,188,189,245,286$

Columbia University

139, 140, 141, 142, 190, 191, 192, 287

Compact Membrane Systems, Inc 435

Connecticut, University of $246,247,288$

Consultec Scientific, Inc. 436

Cornell University $193,194,195,196,406,407,408$

Dartmouth College 143

Delaware, University of 289, 290, 368, 409

Duke University 369

EIC Laboratories, Inc. 437

Emory University 197, 198

Florida State University 199,400

Florida, University of 370,371

George Washington University, The 372

Georgia Institute of Technology 144,248

Georgia, University of 200,201

Hampton University 373

Harvard University $202,203,249,291$

Houston, University of 145

Idaho National Engineering Laboratory 92

Mlinois, University of, at Chicago 204,410

nlinois, University of, at Urbana-Champaign $205,292,374$

Indiana University $293,294,295$

Iowa, University of 296

Johns Hopkins University 206, 207, 411

Kansas State University 208, 250, 297, 375

Kansas, University of 251

Kentucky, University of $209,252,253$

Lawrence Berkeley Laboratory, University of California, Berkeley

$13,14,38,39,40,41,52,53,54,72,73,74,75,76,93,107,108$ $112,113,121,122,123$ 
Lawrence Livermore National Laboratory, University of California

$$
42,55
$$

Lehigh University

$298,299,376$

Los Alamos National Laboratory, University of California $77,109,110,114,124$

Louisiana State University. $300,301,377$

Louisville, University of 254, 302

Marquette University 146

Maryland, University of, at College Park 303,412

Massachusetts Institute of Technology $210,211,304,305$

Massachusetts, University of, at Amherst 212, 213,306, 413

Massachusetts-Boston, University of 147

Materials and Electrochemical Research Corp. 438

Membrane Technology \& Research, Inc. 439

Memphis, University of 307

Michigan State University 378

Michigan Technological University: 255

Michigan, University of 214,308

Microsensor Systems, Inc. 440

Minnesota, University of $148,149,215,216,309,425,426$

Missouri, University of, at Columbia, 310

Missouri, University of, at Rolla 379.

Moltech Corporation 427

National Institute for Petroleum and Energy Research 115.

National Institute of Standards and Technology, Boulder 414

National Insțitute of Standards and Technology, Gaithers; burg $150,217,218,219,380$

National Renewable Energy.Laboratory $15,16,17,18,19,20,78,125$ :

Nebraska, University of, at Eincoln 256

Nevada, University of, at Las Vegas 428

Nevada, University, of, at Reno, 257

New Mexico, University of 258,401

New Orleans, University of $151 ; 220$

New York University. 221

New York, City College of 259

North Carolina State University. $415 ; 429$

North Carolina, University of $f_{2}$ at ChapelpHill $152,222,311 ; 312,381$

North Dakota.State.University 223:

Northwestern University. $153,154,313,314,315,316$
Notre Dame Radiation Laboratory, University of Notre Dame

$21,22,23,24,25,26,27,28,29$

Notre Dame, University of 260 .

Oak Ridge National Laboratory

$56,57,58,59,79,80,81,82,83,94,95,96,97,98,99,100,101$, $102,111,126$,

Ohio State University

155,402

Oklahoma, University of

317

Oregon, University of: 156, 224

Pacific Northwest Laboratory

$43,44,84,103,104,105$

Pennsylvania State University, Lehman 261

Pennsylvania State University, University Park $157,158,225,318,319,320,321,430$.

Pennsylvania, University of $159,226,227,322,323,324,325,416$

Pittsburgh, University of $160,326,327$

Portland State University 161

Princeton University: $162 ; 228,229,230,417$

Purdue University:

$328,329,330,382,383$

Rensselaer Polytechnic Institute 331, 384

Rice University $231,262$.

Rochester, University of $163,164,165,232,332,333$

Rutgers, The State University of New Jersey $166,334,335,431$

Sandia National Laboratories, California $45,46,47,48,49,116$

- Sandia National Laboratories, New Mexico 127:

South Carolina, University of 336 .

Southern California, University of $233,234,235,263,264,337$

Stanford University $167,236,338,418$

State University of New, York at Binghamton 339.

State University of New. York at Buffalo 340,385 .

State. University of New York at Stony Brook 237, 419

Syracuse University. 341,386

TDA Research, Inc. 441

Tennessee, University of, at, Knoxville $168,265,387,388,389,420$

Texas A \& M, University $342,343,344,390,432$

Texas Tech University: 391,392

Texas, University, of; at Arlington, 169

Texas, University, of, at:Austin. $170,171,266,345,346,393$

Toledo, University, of. 267,.268.

Tulane University $172 ; 269 ; 3477_{i}$

Utah, University of $238,348,349,394,395,396$ 
Vanderbilt University 270

Virginia Commonwealth University 397

Virginia Polytechnic Institute and State University 350

Virginia, University of 271,351

Washington State University $173,174,398$

Washington, University of $175,239,352,353$

Wayne State University 176

Western Michigan University 272,273
Wichita State University

177

William and Mary, The College of 274

Wisconsin, University of, at Madison $240,241,354,355,356$

Wisconsin, University of, at Milwaukee 357,358

Wyoming, University of 399

Yale University $359,360,361,421$ 



\section{INVESTIGATOR INDEX}

Investigators' surnames are indexed in the form appearing in the citation; given names generally have been reduced to initials. For projects with multiple investigators, each name is indexed. Accent marks are not input because of computer alphabetization. Spelling and transliteration follow standard conventions.

Abraham, K.M., 437

Adams, R.D., 336

Adzic, R., 90

Alexandratos, S.D., 387

Alnajjar, M.S., 84

Alston, S.G., 261

Andersen, R.A., 107

Anderson, K.B., $68-69$

Andrews, M.A., 71

Angelici, R.J., 60

Angell, C.A., 422

Appelhans, A.D., 92

Appelman, E.H., 106

Armentrout, P.B., 238

Armstrong, D.W., 379

Ashurst, W., 116

Asmus, K.D., 21

Atwood, J.D., 340

Auerbach, D.J., 184

Autrey, T., 84

Baer, T., 222

Baker, G.L., 429

Baker, T.K., 320

Balasubramanian, $K$, 180

Barbara, P.F., 148

Barker, J.R., 214

Barkigia, K.M., 9

Barlow, R.S., 45

Barnes, M.D., 98

Barrans, R.E., 89

Barshick, C.M., 99

Barteau, M.A., 289

Bartels, D.M., 7

Bartlett, N., 72

Bartsch, R.A., 391

Basaran, O.A., 96

Bates, J.B., 126

Beck, D.R., 255

Becker, K.H., 259

Beiersdorfer, P., 55

Bein, T., 328

Beitz, J.V., 106

Belfort, G., 384

Belkacem, A., 53

Bell, A.T., 73

Bentley, J., 22

Bercaw, J.E., 277

Bergman, R.G., 74

Berrah, N., 272

Berry, D.H., 323

Bersohn, R., 190

Beuhler, R.J., 37

Bohn, P.W., 374

Bonneson, P.V., 102

Botto, R.E., 69

Bowman, C.T., 236

Bowman, J.M., 197

Bradshaw, J.S., 364

Braun, C.L., 143

Brezinsky, K., 230

Bright, F.V., 385

Britt, P.F., 80
Brookhart, M., 311

Brown, N.J., 41

Brunschwig, B.S., 12

Bryan, J.C., 102

Bryant, H.C., 258

Buchanan, A.C., 80

Bullock, R.M., 71

Burns, C.J., 77, 109

Bursten, B.E., 402

Bushaw, B.A., 105

Butler, L.J., 186

Byers, C.H., 96

Cabelli, D. E., 11

Cairns, E.J., 122

Calvin, M., 13

Camaioni, D.M., 84

Campbell, C.T., 352

Caradonna, J.P., 360

Carmichael, I.C., 22, 28

Carrado, K.A., 69, 119

Casey, C.P., 355

Castleman, A.W., Jr., 225

Castner, E.W., 12

Caulton, K.G., 293

Cavagnero, M.J., 252

Cavanagh, R.R., 217

Ceyer, S.T., 304

Champion, R.L., 274

Chandler, D., 135

Chandler, D.W., 46

Chateauneuf, J. E., 29

Chen, J.H., 45

Chen, L.X., 5

Chen, M.J., 67

Chen, P., 203

Cheng, R.K., 113

Chiarizia, R., 89

Chipman, D.M., 22

Chisholm, M.H., 294

Choppin, G.R., 400

Christou, G., 295

Chu, S.I., 251

Chumanov, G., 1

Cioslowski, J., 199

Clark, D.L., 110

Clouthier, D.J., 209

Cochran, H.D., 94

Cohen, N., 178

Colson, S.D., 43

Commins, E.E., 52

Conner, W.C., 306

Cooke, W.E., 263

Cooks, R.G., 382

Cool, T.A., 193

Cotton, T.M., 1

Cox, D.F., 350

Crabtree, R.H., 361

Creutz, C., 12

Crim, F.F., 240

Crosby, G.A., 173

Cummings, P.T., 420

Cundari, T.R., 307
Curl, R.F., Jr., 231

Curtis, C.J., 78

Curtis, L.J., 267

Curtiss, L.A., 4

Cutler, A.R., 331

d'Itri, J.L., 326

Dabestani, R.T., 83

Dahl, D.A., 92

Dai, H.L., 226

Dalgarno, A., 249

Dasgupta, P.K., 392

Datz, S., 56

Davis, M., 34

Davis, R. J., 351

Debenedetti, P.G., 417

Delmore, J.E., 92

DePristo, A.E., 30

Dickinson, J.T., 398

Diebold, G.J., 365

DiMauro, L., 37

Donohue, M.D., 411

Doughty, D.H., 127

Doverspike, L.D., 274

Dryer, F.L., 229

DuBois, D.L., 78

DuBois, M.R., 286

Duckworth, D.C., 99

Dumesic, J.A., 354

Duncan, M.A., 200

Dunford, R.W., 50

Dunning, T.H., Jr., 44

Durant, J.L., 46

Dutta, P.K., 155

Dyrkacz, G.R., 69

Edelstein, N.M., 107

Eisenberg, R., 163

Eisenthal, K.B., 139

Ekerdt, J.G., 345

El9Sayed, M.A., 144

Elliott, C.M., 136

Ellis, T.W., 117

Ellison, G.B., 188

Ely, J.F., 404

Endicott, J.F., 176

Erpenbeck, J.J., 114

Espenson, J.H., 61

Evans, W.J., 279

Eyring, E.M., 394

Fajer, J., 9

Farrar, J.M., 232

Farrow, R.L., 47

Fateley, W.G., 375

Fayer, M.D., 167

Feagin, J.M., 243

Fedkiw, P.S., 429

Feldberg, S.W., 10

Felker, P.M., 183

Ferraudi, G.J., 23

Fessenden, R.W., 24

Field, R.W., 211

Finke, R.G., 284

Fischer, C.F., 270 
Flynn, G.W., 191

Ford, P.C., 282

Fox, M.A., 170

Frank, A.J., 15

Franz, J.A., 84

Frei, H.M., 14

Freiser, B.S., 383

Friend, C.M., 291

Friend, D.G., 414

Friesner, R.A., 140

Fripiat, J., 357

Fritz, J.S., 85

Fujita, E., 12

Fulton, J.L., 104

Fultz, B., 423

Fung, K.H., 91

Gallagher, T.F., 271

Garofalini, S.H., 431

Garrett, B.C., 44

Gates, B.C., 278

Gemmell, D.S., 50-51

Gentry, W.R., 215

Gibson, D.H., 302

Gibson, J.K., 111

I Giese, C.F., 215

Gladysz, J.A., 348

Gland, J.L., 308

Glandt, E.D., 416

Glass, G.P., 231

Glassman, I., 230

Goeringer, D.E., 101

Goldman, A.S., 334

Gonzalez, R.D., 347

Goodman, D.W., 342

Gordon, M., 30

Gordon, R.L., 103

Gorte, R.J., 322

Gottlieb, C., 202

Gould, H.A., 53

Gould, P.L., 246

Grant, D.M., 349

Gray, S., 34

Greenbaum, E., 79

Greene, C.H., 245

Gregg, B.A, 16

Gregorich, K.E., 108

Grimm, F.A., 82

Gubbins, K.E., 406

Guiochon, G., 388

Guldi, D.M., 21

Gust, J.D., Jr., 130

Hagaman, E.W., 80

Hahn, Y., 247

Haire, R.G., 111

Fall, C.K., 415

Hall, G.E., 36

Hall, K.W., 326

Hallex, G.L., 359

Halley, J.W., 426

Hammaker, R.M., 375

Hanley, H.J.M., 414

Hanson, R.K., 236

Harding, L., 34

Harmin, D.A., 252

Harris, C.B., 38

Harris, J.M., 395

Harrison, W., 370

Havener, C.C., 57

Haw, J.F., 343

Hayden, C.C., 46

Hayes, J.M., 2

Heinekey, M., 353

Heller, E., 239

Helman, W.P., 28
Herman, R.G., 299

Hershberger, J.F., 223

Hessler, J., 34

Ho, P.C., 81

Ho, W., 194

Hoffman, D.C., 108

Hoffman, M.Z., 131

Holroyd, R.A., 11

Homsy, G.M., 418

Horwitz, E.P., 89

Hougen, J.T., 219

Houk, R.S., 86

Houston, P.L., 195

Howard, J.B., 210

Hrbek, J., 71

Hug, G.L., 28

Hulett, L.D., Jr., 97

Hunt, R.D., 95

Hunter, S.R., 436

Huntley, D.R., 82

Hupp, J.T., 153

Hurst, J.K., 174

Hurtubise, R.J., 399

Ipatieff, V.N., 315

Isied, S.S., 166

Izatt, R.M., 364

Jackson, B.E., 213

Jellinek, J., 32

Johnson, E., 111

Johnson, J.R., 70

Johnson, P.M., 237

Johnston, H.S., 39

Jonah, C.D., 7

Jones, G., 132

Jones, W.D., 333

Jonsson, H., 239

Jordan, R.F., 296

Kamat, P.V., 25

Kanter, E.P., 50-51

Kee, R.J., 48

Kellman, M.E., 224

Kern, R.D., Jr., 220

Kerstein, A.R., 116

Keto, J.W., 266

Kevan, L., 145

Khan, S.A., 429

Kiefer, J.H., 204

Kielkopf, J., 254

Kimble, H.J., 242

Kincaid, J.R., 146

King, D.S., 217

King, T.S., 62

Kinoshita, K., 123

Kispert, L.D., 129

Klemm, R.B., 35

Klier, K., 299

Klingler, R.J., 67, 118

Knickelbein, M.B., 32

Ko, E.I., 283

Koel, B.E., 337

Koetzle, T.F., 71

Koffend, J.B., 178

Koros, W., 393

Kostroun, V.O., 196

Koval, C.A., 138

Krause, H.F., 56

Kubas, G.J., 77

Kung, H.H., 313

Kvale, T.J., 268

Lamb, J.D., 364

Landis, C.R., 356

Laurence, R.L., 306

LaVerne, J.A., 26

Lee, Y.T., 39
Lees, A.J., 339

Lenz, T.G., 405

Leone, S.R., 189

Lester, M.I., 227

Lester, W.A., 39

Lewis, N.S., 134

Libby, P.A., 403

Licht, S., 424

Lichtenberger, D.L., 182

Light, J.C., 187

Lim, E.C., 128

Lin, C.D., 250

Lin, M.C., 198

Linehan, J.C., 84

Linschitz, H., 133

Lipsky, S., 149

Livingston, A.E., 260

Long, M.B., 421

Loutfy, R.O., 438

Lucht, R.P., 205

Lunsford, J.H., 344

Maatta, E., 297

Macdonald, D.D., 430

MacDonald, R., 34

Maciel, G.E., 367

Madden, K.P., 24

Madey, T.E., 335

Madix, R.J., 338

Mallouk, T.E., 157

Marks, T.J., 314

Maroncelli, M., 158

Marrs, R., 55

Martin, B.G., 434

Martin, N.L.S., 253

McBreen, J., 120

McDermott, A.E., 141

McGown, L.B., 369

McGuffin, V.L., 378

McGuire, J.H., 269

McLuckey, S.A., 101

McMahon, J.M., 100

Meisel, D., 4

Mesmer, R.E., 81

Meyer, F.W., 57

Meyer, T.J., 152

Michael, J., 34

Mícic, O.I., 17

Miller, G.J., 63

Miller, J.A., 46, 48

Miller, J.D., 396

Miller, J.R., 4

Miller, R.J.D., 164

Miller, W.H., 39

Monchick, L., 207

Moncrieff, D., 199

Monson, P.A., 413

Montaser, A., 372

Moore, A.L., 130

Moore, C.B., 39

Moore, T.A., 130

Morss, L.R., 106

Moyer, B.A., 102

Mozumder, A., 26

Msezane, A.Z., 244

Muckerman, J.T., 36

Mullins, D.R., 82

Murad, S., 410

Murray, R.W., 381

Nagarajan, V., 175

Najm, H., 45

Nash, K.L., 106

Neaser, S., 435

Neta, P., 150

Neuman, R.D., 363 
Neumark, D., 39

Newton, M.D., 12

Ng, C.Y., 31

Nicholas, K.M., 317

Norris, J.R., 5-6

Norton, J.R., 285

Nozik, A.J., 18-19

Osgood, R.M., Jr., 142

Otvos, J.W., 13

Overbury, S.H., 82

Paine, R.T., 401

Painter, P.C., 321

Palmer, D.A., 81

Panagiotopoulos, A.Z., 407

Parkin, G., 287

Parkinson, B.A., 137

Parks, E.K., 32

Patterson, L. K., 29

Patterson, L.K., 27

Paul, D., 393

Paul, P.H., 45, 49

Pegg, D.J., 265

Pemberton, J.E., 362

Perry, D.S., 179

Peterson, J.R., 111

Pimblott, S.M., 26

Pinnau, I., 439

Piotrowiak, P., 151

Pitzer, K.S., 40

Poli, R., 303

Pope, S.B., 408

Porter, M.D., 87

Prausnitz, J. M., 112

Preses, J., 36

Price, G.L., 300

Prior, M.H., 54

Pruski, M., 64

Pugmire, R.J., 349

Rabideau, P.W., 301

Rabitz, H.A., 228

Ragle, J.L., 306

Rajeshwar, K., 169

Rakestraw, D.J., 47-48

Ramsey, J.M., 98

Ramsey, R.A., 101

Rathke, J.W., 67

Ratnakumar, B.V., 423

Rauchfuss, T.B., 292

Raymond, K.N., 107

Reddy, N.R.S., 2

Regen, S.L., 376

Reilly, J.J., 70, 120

Reinhold, C.O., 58

Reisler, H., 233

Renner, M.W., 9

Richard, P., 208

Riciputi, L.R., 99

Riley, S.J., 32

Rillema, D.P., 177

Rodriguez, J.A., 71

Rohlfing, E.A., 46

Ross, A.B., 28

Ross, P.N., Jr., 121

Rothwell, I.P., 329

Roy, R., 248

Ruedenberg, K., 30

Ruscic, B., 33

Russell, D.H., 390

Russo, R.E., 93

Rutan, S.C., 397

Sachleben, R.A., 102
Sachtler, W.M.H., 315

Saltsburg, H., 332

Sandler, S.I., 409

Sauer, M.C., Jr., 7

Savikhin, S., 3

Schaefer, H.F., III, 201

Schefer, R.W., 45

Schmehl, R.H., 172

Schmidt, L.D., 309

Schneider, D., 55

Schrader, G.L., 65

Schrock, R.R., 305

Schuler, R.H., 26, 29

Schultz, D.R., 58

Schwarz, H.A., 11

Schwarz, J.A., 341

Schwarz, R.B., 124

Scott, L.T., 276

Sears, T.J., 36

Seibert, M., 20

Selser, J.C., 428

Seltzer, S., 12

Sen, A., 319

Sengers, J.V., 412

Sepaniak, M.J., 389

Shakeshaft, R., 264

Sharp, P.R., 310

Shaw, R.W., 98

Shepard, R., 34

Shirts, R.B., 364

Short, R.T., 100

Shriver, D.F., 316

Shuh, D.K., 107

Sieck, L.W., 380

Sigman, M.E., 83

Silbey, R.J.; 211

Simmons, G.W., 299

Simonson, J.M., 81

Skotheim, T., 427

Slagle, I., 185

Small, G.J., 2

Smalley, J.F., 10

Smith, D.H., 99

Smooke, M.D., 421

Smyrl, W.H., 425

Sneddon, L.G., 324

Soderholm, L., 106

Spears, K.G., 154

Spiro, T.G., 162

Squires, R.R., 330

Srinivas, G., 441

Srinivasan, S., 432

Starace, A.F., 256

Steele, W.V.; 115

Steimle, T.C., 181

Stell, G.R., 419

Stern, S.A., 386

Stock, L.M.; 69

Strayer, M., 58

Striebel, K.A., 122

Struve, W.S., 3

Styris, D.L., 103

Suib, S.L., 288

Suits, A., 39

Sutherland, J.W., 35

Sutin, N., 12

Taatjes, C.A., 46

Talbot, L., 113

Tang, I.N., 91

Tang, J., 5

Tanis, J.A., 273
Taylor, H. S., 234

Templeton, J.L., 312

Thaddeus, P., 202

Theopold, K.H., 290

Therien, M.J., 159

Thurnauer, M.C., 5

Tiede, D.M., 5-6

Tilley, T.D., 75

Todd, P.J., 100

Toth, L.M., 95

Trahanovsky, W.S., 66

Trebino, R:, 49

Trifunac, A.D., 7

Tripathi, G.N.R., 29

Truhlar, D.G., 216

Tsang, W., 218

Turner, J.A., 125

Tyler; D.R., 156

Tysoe, W.T., 358

Urasa, I.T., 373

Valentini; J.J., 192

van Willigen, $\mathbf{H}$., 147

VanBerkel, G.J., 101

Vane, C.R., 56, 59

Vannice, M.A., 318

Verdine, G.L.; 203

Vollhardt, K.P.C., 76

Wachs, İ.E., 298

Wagner, A.; 34

Waldeck, D.H., 160

Wamser, C.C., 161

Warner, I.M., 377

Wasielewski, M.R., 5, 8

Way, J.D., 366

Wayland, B.B., 325

Webber, S.E., 171

Weinberg, W.H.; 281

Weisheit, J.C., 262

Weisshaar, J.C., 241

Werst, D.W., 7

Westbrook, C.K., 42

Westmoreland, P.R., 212

White, J.M., 346

White, M., 37

Whitehead, A., 105

Whitten, D.G., 165

Whitten, W.B., 98

Wiederrecht, G.P., 8

Wigley, D.E., 275

Williams, T.F.; 168

Winans, R.E.; 69, 119

Winefordner, J.D., 371

Winkler, P., 257

Winiter, T.G., 261

Wirth, M.J., 368

Wishart, J.F., 11

Wittig, C., 235

Wodtke, A.M., 184

Wohltjen, H., 440

Wojciechowski, M., 433

Woodward, J., 79

Yarkony, D.R., 206

Yates, J.T., Jr., 327

Yeung, E.S., 88

Yonker, C.R., 104

Young, J.P., 98

Young, L., 50-51

Zaera, F., 280

Zhang, J.G., 125 